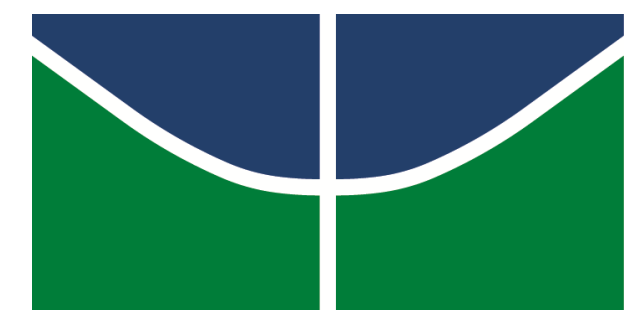

$$
\begin{gathered}
\text { Universidade de Brasília } \\
\text { Instituto de Ciências Biológicas } \\
\text { Programa de Pós-graduação em Ecologia }
\end{gathered}
$$

TESE DE DOUTORADO

PADRÕES BIOGEOGRÁFICOS E COMPOSIÇÃO DAS COMUNIDADES ARBÓREAS DO CERRADO BRASILEIRO

RENATA DIAS FRANÇOSO

ORIENTADOR: PROF. DR. RICARDO BOMFIM MACHADO

Brasília - DF

Dezembro, 2014 


$$
\begin{gathered}
\text { Universidade de Brasília } \\
\text { Instituto de Ciências Biológicas } \\
\text { Programa de Pós-graduação em Ecologia }
\end{gathered}
$$

TESE DE DOUTORADO

\section{PADRÕES BIOGEOGRÁFICOS E COMPOSIÇÃO DAS COMUNIDADES ARBÓREAS DO CERRADO BRASILEIRO}

\section{RENATA DIAS FRANÇOSO}

Tese apresentada ao Programa de Pós-Graduação em Ecologia, Instituto de Ciências Biológicas da Universidade de Brasília, como parte dos requisitos necessários para a obtenção do título de Doutora em Ecologia.

ORIENTADOR:

PROF. DR. RICARDO BOMFIM MACHADO

$$
\text { Brasília - DF }
$$

Dezembro, 2014 
Tese de Doutorado

\section{RENATA DIAS FRANÇOSO}

Título:

\section{"Padrões biogeográficos e composição das comunidades arbóreas do} Cerrado brasileiro"

\section{Banca Examinadora:}

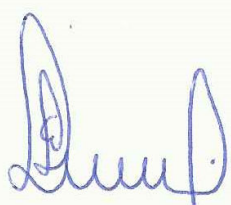

Prof. Dr. Eddie Lenza Membro Titular UNEMAT<smiles>C=CCCCCCCCCCC</smiles>

Prof. Dr. Marcelo Simon Membro Titular Embrapa/Cenargen

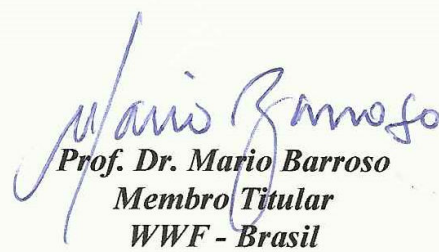

Prof. Dr. José Roberto R. Pinto Membro Titular $E F L / U n B$

Prof. Dr. John Du Vall Hay

Suplente

$E C L / U n B$

Brasília, 09 de dezembro de 2014. 
Dedico aos estudiosos do Cerrado 


\section{AGRADECIMENTOS}

Nem acredito que está no fim! Quatro anos nunca renderam tanto! Gostei muito de desenvolver esse trabalho, e espero seguir fazendo o que gosto. Confesso que já sinto um misto de alívio e nostalgia.

Agradeço à minha família (mãe, pai, tios, primos, avós) por me apoiar nas minhas decisões, mesmo com tantas incertezas, tão poucas oportunidades de trabalho e tanta cobrança. Tantas, que muitas vezes dá vontade de desistir. Nesses momentos minha família esteve por perto, pronta para me ajudar a sacodir a poeira! Agradeço especialmente à minha mãe, que sempre acreditou em mim, muito mais do que eu mesma.

Agradeço muito ao meu marido, Reuber Brandão, por estar sempre ao meu lado, aguentando meus chiliques, mas também torcendo e comemorando minhas conquistas. Seu apoio é muito importante para mim. Não posso esquecer de agradecê-lo por ter me acompanhado no meu primeiro campo do doutorado.

Também agradeço ao Pacheco, meu orientador. Eu já era bolsista do seu laboratório e decidi fazer o doutorado em Ecologia. Para mim não havia melhor escolha do que continuar a trabalhar ali, e prontamente o Pacheco aceitou esse desafio. Desafio porque, como Engenheira Florestal, minha formação em ecologia não era lá essas coisas. Além disso, trabalhar com plantas em um laboratório na zoologia sempre causa estranhamento. No entanto, a ampla experiência do Pacheco com a biodiversidade e conservação do Cerrado, e seu arsenal de ferramentas e análises espaciais, contribuíram muito com a minha formação e com o desenvolvimento desse estudo.

Pela ótima recepção e colaboração, agradeço ao trio de orientadores do período sanduíche no Royal Botanic Garden Edinburgh: Toby Pennington, Kyle Dexter e Jimmy Ratter. Estendo os agradecimentos ao pessoal do Tropical Diversity Group, especialmente aos colegas Roosvelt, Maca, Subhani, Peter, Julieth, Karina e Lidiany. O apoio desse grupo foi incrível. Como diria o Jimmy "Não poderia ser melhor!". Aliás, não tenho como passar tão rapidamente pelo Jimmy. Seu trabalho no Cerrado foi, sem dúvida, a inspiração dessa tese. Quando estava ingressando no doutorado, nem imaginava que o teria na minha banca de qualificação. Tampouco que eu passaria meses desfrutando da sua companhia. 
Agradeço aos professores do programa, pela sólida formação que recebemos. Também agradeço à Vanessa, pelo apoio sempre eficiente! Agradeço também à prof. Ludmilla, pela agradável companhia nos corredores da Zoologia! E não poderia me esquecer de agradecer ao prof. Roberto Cavalcante, que tentando me preparar para a entrevista da seleção do doutorado, quase acabou com todas as minhas chances, de tantas perguntas capciosas que fez!

Agradeço à equipe que me ajudou em campo: Izabel Oliveira, Daniel Carneiro, Rafael Ataídes, Fernando Vieira e nosso "chefe" Ricardo Haidar. Aprendi muito com esse pessoal! Agradeço especialmente à Izabel, pelas horas que passou em frente ao computador compilando dados e revisando nomenclatura, e ao Ricardo, que me ensinou muito sobre as plantas do Cerrado. Além disso, cedeu generosamente uma parte importante da base de dados que usei nessa tese.

Agradeço aos colegas do LaBio, o laboratório mais bonito do Brasil: Bárbara Zimbres, Renata Alquezar, Vivian Ribeiro, Giovana Bottura, Fernando Goulart, Edsel, Danilo Oliveira, Fernanda Figueiredo, Mariana Stein, Yuri Salmona, Romina Cardoso e Thalita Grande com quem compartilhei muitos momentos e experiências. As discussões, quando não muito acaloradas, foram bastante produtivas!

Agradeço aos amigos da zoologia: Ana M., Emília \& Eduardo, Samuca, Pedro \& Marina, Clarice \& Alexandre, Ingrid, Léo \& Leozinho, Danilo, André \& Liliam, Gabriel, Luane \& Neander, Thiago, Ricardo, Juliana, Guibam, Cecília \& Guarino, Ana H., Ana Parceira, Jéssica \& Léo, Pedro, Marcela \& Renan, Isis, Guth, Gabriel, Helga, Davi, Verônica e galera supracitada do LaBio pelos ótimos momentos! Os mais produtivos foram pelos botecos da cidade!

Sem mais delongas, vamos ao que interessa! 


\section{SUMÁRIO}

RESUMO
ABSTRACT
CAPÍTULO 1. FLORA ARBÓREA DO CERRADO BRASILEIRO: SÍNTESE DO CONHECIMENTO,

INFLUÊNCIA DOS BIOMAS VIZINHOS E LACUNAS DE INFORMAÇÃO

RESUMO

INTRODUÇÃO

MÉTODOS

RESULTADOS

DISCUSSÃO

AGRADECIMENTOS

REFERÊNCIAS

FIGURAS

TABELAS

MATERIAL SUPLEMENTAR 1

MATERIAL SUPLEMENTAR 2

CAPÍTULO 2. DELIMITAÇÃO E CARACTERIZAÇÃO DOS DISTRITOS BIOGEOGRÁFICOS DO CERRADO

RESUMO

INTRODUÇÃO

MÉTODOS

RESULTADOS

DISCUSSÃO

AGRADECIMENTOS

REFERÊNCIAS

FIGURAS

TABELAS

MATERIAL SUPLEMENTAR 1

CAPÍTULO 3. CONTRIBUIÇÃO DE FATORES CLIMÁTICOS E EDÁFICOS PARA A DISTRIBUIÇÃO DAS ESPÉCIES LENHOSAS DO CERRADO

RESUMO

INTRODUÇÃO

MÉTODOS

RESULTADOS

DISCUSSÃO 
AGRADECIMENTOS

REFERÊNCIAS

FIGURAS

TABELAS

MATERIAL SUPLEMENTAR 1

MATERIAL SUPLEMENTAR 2 
O conhecimento sobre a biodiversidade e descrição dos seus padrões são as abordagens primordiais da ecologia e da biologia da conservação. Entender como os organismos se distribuem na paisagem e quais são seus determinantes ambientais, é fundamental para a formulação de políticas de conservação. O Cerrado brasileiro é um hotspot de biodiversidade, no entanto há muitos déficits de conhecimento nesse ecossistema. No primeiro capítulo, realizamos uma síntese de 167 estudos de levantamentos florísticos e fitossociológicos de cerrado sentido amplo, totalizando 909 espécies arbóreas, 298 gêneros e 76 famílias. Observamos maior riqueza local nas regiões próximas ao centro do bioma, porém, devido à alta diversidade beta, as regiões mais distantes do centro do Cerrado apresentaram maior riqueza acumulada de espécies. A Mata Atlântica foi o bioma mais importante para composição florística do Cerrado, e a proporção de espécies endêmicas é de apenas 5\%, por isso novos estudos da flora devem incluir outras formas de vida, como ervas, lianas, arbustos e subarbustos, onde devem estar as maiores proporções de espécies endêmicas. O segundo capítulo é uma atualização dos Distritos Biogeográficos (DB) do Cerrado com base na similaridade de comunidades de plantas lenhosas. Usando uma base de dados ampliada e revisada, caracterizamos os DB em relação ao clima, espécies indicadoras e estado de conservação. Identificamos sete $\mathrm{DB}$, dos quais três $\mathrm{DB}$ localizados nas regiões extremas do Cerrado, apresentaram maiores dissimilaridades em relação aos demais. Esses DB tiveram grande influência dos biomas adjacentes. Os maiores números de espécies típicas de cerrado foram observados nos DB Centro-oeste, que possui maior extensão, Centro, que corresponde às áreas altas do Cerrado, e Nordeste, que é a região de maior estabilidade climática desde as glaciações do Quaternário. Temperatura mínima anual, sazonalidade da temperatura e precipitação anual são as variáveis mais importantes para a diferenciação dos grupos, que apresentaram dois principais padrões climáticos: os DB Sul, DB Sudeste e DB Sudoeste possuem oeste, DB Norte e DB Nordeste apresentaram padrão oposto. O DB Centro apresentou tanto baixas temperaturas mínimas, quanto baixa sazonalidade da temperatura. Em termos de conservação, os DB apresentam grande variação nos níveis de desmatamento e de áreas protegidas. A delimitação dos DB, aliados a ferramentas e técnicas consolidadas da biologia da conservação, podem servir de ponto de partida para equilibrar os esforços de conservação do bioma Cerrado. No terceiro capítulo estudamos a influencia dos filtros ambientais na 


\section{.}

distribuição das espécies arbóreas do Cerrado. O clima e os solos são os filtros mais importantes para a distribuição das plantas, atuando como uma força seletiva dos traços das espécies. No entanto, essas características podem ter origem histórica, quando provem de um ancestral comum que partilhava a mesma característica, ou ecológica, quando por pressão externa, espécies de diferentes linhagens convergem em traços semelhantes. No Cerrado muita ênfase tem sido dada ao papel do solo na diferenciação das comunidades, mas não se sabe ao certo sua importância, por isso comparamos a importância de variáveis climáticas e edáficas na composição das comunidades lenhosas do cerrado, e verificamso se há conservatismo de nicho em relação às variáveis mais importantes. Para isso realizamos uma análise de partição de variância em três escalas, usando 87 levantamentos florísticos, 11 variáveis climáticas e 11 variáveis edáficas. Testamos individualmente a importância dessas variáveis para a ocorrência de cada uma das 303 espécies usando uma regressão logística. O clima foi mais importante para a determinação da comunidade nas escalas mais amplas, no entanto na menor escala e nas análises específicas o solo teve maior contribuição, aumentando a heterogeneidade em escala regional. Dentre as variáveis mais importantes, apenas preferência por soma de bases é uma característica conservada na filogenia, o que já foi demonstrado em outros ecossistemas. Estudos sobre potencial de dispersão das árvores do cerrado são importantes no presente cenário de mudanças climáticas.

Palavras-chave: florística, cerrado sentido amplo, plantas lenhosas, biomas brasileiros, fitogeografia, distritos biogeográficos, filtros ambientais. 
The knowledge on biodiversity and the descriptions of its patterns are elementary approaches in Ecology and Conservation Biology. Understanding how the organisms are settled on the landscape and why they occupy those places is essential for the conservation policy makers. The Brazilian Cerrado is a biodiversity hotspot, however there are many gaps in its knowledge. In the first chapter we summarized 167 floristic and phytosociological studies of cerrado lato sensu, summing 909 tree species, 298 genus, and 76 families. We observed great richness in the regions near to the center, but, due to the high beta diversity, the most distant regions to the center have the great cumulative richness. The Atlantic Forest is the most important biome for the Cerrado's floristic composition, and the proportion of endemic species is just $5 \%$. Therefore, new studies must includ other life forms, as herbs, lianas, shrubs, and subshrubs, since they must have a greater proportion of endemism. The second chapter is an update of the Biogeographic Districts (BD) of Cerrado biome, based on community similarity of wood species. Using an expanded and revised database, we described the BD regarding the climate, indicator species, and conservation status. We identified seven BD, three of them, on Cerrado's edge, have greater dissimilarity in relation to the other. Those BD have great influence of the adjacent biomes. We observed a great number of Cerrados typical species in three of the BD: in Center West because it's big area, in Center, because it corresponds to the high lands of Cerrado; and North West, which is the most climatically stable area since the Quaternary glaciation. Minimum anual temperature, temperature sazonality and annual precipitation are the most important variables to discriminate the $\mathrm{BD}$, wich presented two main climatic patterns: The BDs South, South East and South West have lower temperature and greater temperature; and the BDs Center West, North and North East have the opposite pattern. The BD Center has both low minimum temperature as low temperature sazonality. About the conservation status, the BDs presented a great variation in deforestation levels and in protected areas. The delimitation of the BDs, with other tools and techniques of Conservation Biology, can be the starting point to balance the conservation efforts in the Cerrado bioma. In the third chapter, we studied the influence of environmental filters on the wood species distribution in Cerrado. The climate and soils are the most important filters for the plants, acting as a selective power for the traits. However, those traits may have historical origin, if they come from a common ancestor, or ecological, when, by external pressure, the 
species of different lineages converge in similar traits. In Cerrado vegetation, much emphasis has been given to the soil role, but it's real importance is unknown, therefore we compared the importance of climatic and edaphic variables in community composition of wood trees in Cerrado, and we looked for niche conservatism in the most important variables. Thereunto we proceed a variance partition analysis in three different scales, using 87 floristic surveys, 11 climatic and 11 edaphic variables. We tested the individual importance of those variables for the occurence of those 303 species with a logistic regression. The climate was more important to determine the community composition in the broad scale, but in the smaller scale and in the individual analysis level, the soil was more important, increasing the heterogeneity in regional scale. Among the most important variables, only preference on sum of bases is a conserved trait in the phylogeny, what was established in other ecosystems. New studies about potential of dispersion in wood plants are welcome in the recent climate change scenario.

Key-words: floristic, cerrado lato sensu, wood plants, Brazilian biomes, phytogeography, biogeography districts, environmental filters. 


\section{CAPÍTULO 1. FLORA ARBÓREA DO CERRADO BRASILEIRO: SÍNTESE DO CONHECIMENTO, INFLUÊNCIA DOS BIOMAS VIZINHOS E LACUNAS DE \\ INFORMAÇÃO}

\section{RESUMO}

O conhecimento da biodiversidade é de extrema importância para nortear ações de manejo e conservação dos recursos naturais, o que motivou diversas compilações de informações sobre a vegetação do bioma Cerrado. Essa vegetação se mostrou extremamente diversa, devido a fatores ecológicos, como a influência de outras formações vegetais, clima, solos, entre outros. Apesar da importância do Cerrado, ainda existem lacunas de informação em regiões de extrema importância para o entendimento dos processos e padrões existentes. Os objetivos desse estudo foram (1) realizar uma síntese dos estudos florísticos e fitossociológicos de espécies arbóreas do Cerrado, trazendo novas informações florísticas e biogeográficas; (2) estudar os padrões de distribuição e riqueza da flora do Cerrado; e (3) identificar lacunas de amostragem para direcionar novos estudos. Compilamos 167 estudos, totalizando 909 espécies arbóreas, 298 gêneros e 76 famílias. Observamos maior riqueza local nas regiões próximas ao centro do bioma, porém, devido à alta diversidade beta, as regiões mais distantes do centro do Cerrado apresentaram maior riqueza acumulada de espécies. A Mata Atlântica foi o bioma mais importante para composição florística do Cerrado, e a proporção de espécies endêmicas é de apenas $5 \%$.

Palavras-chave: ecótono, diversidade beta, flora do Cerrado, biomas brasileiros. 
A ocorrência de um determinado táxon em um local e em um dado momento histórico é a informação biológica mais elementar (Sousa-Baena et al. 2014), sendo crucial para estudos, manejo e conservação da biodiversidade (Boulinier et al. 1998). Durante as últimas décadas diversos estudos sintetizaram o conhecimento sobre a vegetação do Cerrado (Castro et al. 1999; Heringer et al. 1977; Oliveira-Filho \& Ratter 2002; Ratter \& Dargie 1992; Ratter et al. 2003; Rizzini 1963). Tais estudos tiveram como foco a vegetação arbórea das formações savânicas, que cobre a maior parte do Cerrado (Coutinho 2006). Nas primeiras estimativas, 400 espécies arbóreas foram registradas no Cerrado (Rizzini 1963) e, posteriormente, esses números foram aumentados para cerca de 700 (Heringer et al. 1977; Ratter \& Dargie 1992) e 951 (Bridgewater et al. 2004; Ratter et al. 2003) estimando-se finalmente existir entre 1000 e 2000 espécies de árvores e arbustos (Castro et al. 1999). Esse número corresponde a mais de 11\% de toda a biodiversidade do Cerrado (Mendonça et al. 2008), sendo de extrema importância para o entendimento dos padrões e processos ocorridos nesse bioma. Além disso, a vegetação tem maior importância por atuar como substituto de biodiversidade para a maioria dos grupos de animais (Noss 1996).

A alta diversidade e heterogeneidade florística observada no Cerrado é explicada por fatores bióticos e abióticos, como diferenças edáficas, na frequência de fogo, ou ainda por estocasticidade (Batalha \& Martins 2007; Ratter \& Dargie 1992). Possivelmente por essas e outras razões, há uma alta diversidade beta para plantas no Cerrado (Bridgewater et al. 2004; Felfili et al. 1994; Felfili; Sousa-Silva; et al. 2005; Oliveira-Filho \& Ratter 2002; Ratter et al. 2003), sendo que a contribuição dos cerrados marginais para esse padrão é alta (Castro et al. 1999; Rizzini 1963), pois essas exercem influências marcantes nos padrões biogeográficos do Cerrado (Machado et al. 2008). Essa influência ocorre devido à presença ocasional de espécies predominantemente de outros biomas, que são denominadas espécies acessórias (Heringer et al. 1977; Rizzini 1963).

O Cerrado possui diversos gêneros e espécies comuns à Mata Atlântica e à Floresta Amazônica (Bridgewater et al. 2004; Méio et al. 2003), sendo que a influência da Mata Atlântica é significativamente maior do que da Amazônica (Rizzini 1963), provavelmente devido à tolerância às baixas temperaturas (Méio et al. 2003). No entanto, não se sabe ao certo como é a distribuição das espécies compartilhadas entre o Cerrado e os biomas adjacentes. Pela 
importância dessa influência externa, a forma mais característica do Cerrado possivelmente ocorre na região central do bioma (Castro et al. 1999), onde deve estar a maior parte das espécies endêmicas ou predominantes do Cerrado.

As plantas endêmicas representam $44 \%$ do total listado para o bioma, incluindo todos os hábitos e formações vegetais (Myers et al. 2000). Para as espécies arbóreas das formações savânicas não se sabe ao certo a proporção dessas espécies. No entanto, é estimado que apenas sete gêneros sejam exclusivos no Cerrado, mas 226 espécies são consideradas peculiares do Cerrados (Rizzini 1963). Quando consideradas apenas as espécies predominantes no Cerrado, são observadas 107 espécies, correspondendo a 34\% do total observado por Bridgewater et al. (2004).

Nem todas as espécies observadas nas formações savânicas são predominantes nesse ambiente, havendo muito intercâmbio nas fitofisionomias do Cerrado. Há diversos exemplos de espécies congenéricas nas matas de galeria e no cerrado sentido restrito, o que é consequência de gradiente de espécies entre essas duas fitofisionomias (Hoffmann \& Silva-Júnior 2005). Esse gradiente ecológico sugere que ao longo do tempo evolutivo ocorreram colonizações bidirecionais por espécies de formações florestais e savânicas (Forni-Martins \& Martins 2000), já que há uma contínua flutuação das bordas das florestas com as formações savânicas (Silva et al. 2008). Nas matas secas, que são formações florestais associadas aos solos mais férteis (Oliveira-Filho \& Ratter 1995), também é observada uma flutuação das suas bordas, sendo que há evidências da retração dessa fitofisionomia causada pela expansão de cerrado sentido restrito (Silva et al. 2010). Essas flutuações são graduais e contínuas, e no tempo ecológico, caracterizam a ocorrência ocasional de espécies de determinada fitofisionomia em outra, a qual a espécie é menos tolerante, sendo muitas vezes difícil definir seu hábitat principal.

Apesar da sua importância, o conhecimento da biodiversidade muitas vezes é superficial (Sousa-Baena et al. 2014), fazendo com que políticas de conservação sejam subsidiadas por informações biológicas incompletas, tanto do ponto de vista taxonômico (déficit lineriano), quanto do ponto de vista biogeográfico (déficit wallaciano) (Lomolino et al. 2006). De um modo geral, os esforços de inventários são enviesados para determinados grupos taxonômicos e para regiões próximas aos centros de pesquisa, enquanto outras regiões possuem pouco ou nenhum esforço de coleta (Sousa-Baena et al. 2014). Para o aprofundamento do conhecimento 
biológico é necessário que os esforços de coleta sejam otimizados em decorrência da escassez de recursos financeiros e humanos.

A despeito dos esforços empregados no inventário das espécies lenhosas no Cerrado, ainda restam lacunas de informação (Sousa-Baena et al. 2014), especialmente nas áreas de ecótonos entre o Cerrado e domínios adjacentes. Informações provenientes dessas regiões podem ser extremamente valiosas para o entendimento de processos históricos e biológicos, pois contribuem para a heterogeneidade do Cerrado e representam áreas de intensa troca de espécies com domínios vizinhos (Silva \& Bates 2002).

No intuito de aumentar o conhecimento biológico acerca das espécies arbóreas do Cerrado, realizamos uma compilação e síntese dos estudos florísticos e fitossociológicos de espécies arbóreas do Cerrado, trazendo novas informações a respeito do número de espécies e suas distribuições geográficas. Além disso, identificamos as principais lacunas de informação para esse grupo.

\section{MÉTODOS}

\section{Área de estudo}

O Cerrado é o maior bioma (sensu Clements \& Shelford 1939) brasileiro depois da Amazônia, cobrindo mais de $2.000 .000 \mathrm{Km}^{2}$ (IBGE 2004). Ocupa a região central do Brasil, com encraves na Amazônia, Caatinga e Mata Atlântica. É dividido em três tipos de formações, baseado na estrutura da vegetação: formações campestres, savânicas e florestais (Eiten 1972; Ribeiro \& Walter 1998, 2008). O cerrado sentido amplo engloba o cerrado sentido restrito e o cerradão, pois esse último, apesar de pertencer à formação florestal, floristicamente se assemelha ao cerrado sentido restrito (Ribeiro \& Walter 2008). Dessa forma nossas análises se concentraram em localidades de cerrado sentido amplo (sensu Ribeiro \& Walter 2008, e doravante designado apenas como cerrado) por toda a extensão do Cerrado, incluindo encraves nos outros biomas.

\section{Coleta de dados}

Realizamos uma busca de estudos florísticos e fitossociológicos de espécies lenhosas de cerrado na base de dados dos periódicos da CAPES, Web of Science e no Banco de Teses da 
CAPES de diversas universidades, com várias combinações das palavras-chave: cerrado, savana,

214 florística, fitossociologia, fenologia, arbóreas, lenhosas, vegetação, inventário, bem como os correspondentes em inglês destes termos. Além desses, usamos como ponto de partida a compilação de Ratter et al. (2013) e o Inventário Florestal do Estado do Tocantins (Haidar et al. 2013), além de estudos não publicados.

Como ponto de partida para preencher as lacunas de informação existentes no Cerrado, realizamos inventários sistemáticos da vegetação, de acordo com o Manual de Parcelas Permanentes para o Cerrado e Pantanal (Felfili; Carvalho; et al. 2005) e uma adaptação do método de "levantamento rápido" (Ratter et al., 2003; Walter \& Guarino 2006), que consiste em percorrer uma transeção no ponto de amostragem, registrando todas as espécies arbóreas presentes na área, anotando as espécies em intervalos de 15 minutos até que nesse intervalo não ocorram mais novos registros de espécies anteriormente registradas. Walter \& Guarino (2006) adotaram cinco minutos de intervalo, e concluíram que 40 minutos seriam suficientes para incluir grande numero de espécies, não sendo menos eficiente do que o método de parcelas para o registro de espécies. A distância máxima percorrida por estes autores foi de 831 m. Para garantir o sucesso da amostragem, o levantamento rápido foi adaptado para distâncias percorridas, usando um comprimento de $1000 \mathrm{~m}$ em cada transeção.

\section{Caracterização da base de dados}

A taxonomia e nomenclatura das espécies foram atualizadas de acordo com o APG III, e Flora do Brasil (JBRJ 2014), onde também consultamos as informações sobre forma de vida. Essa etapa foi de extrema importância para a compilação dos dados, já que a base de dados é composta por estudos que usam diferentes métodos e critérios de amostragem. Apenas as espécies indicadas como árvores foram consideradas na presente compilação. Dessa forma, diversas espécies incluídas anteriormente por Ratter et al. (2003) nos levantamentos florísticos não estão listadas no presente estudo. As espécies não identificadas foram excluídas da base de dados.

Para determinar o(s) bioma(s) de ocorrência predominante de uma espécie, usamos as coordenadas procedentes dos herbários, acessadas pelo SpeciesLink para cada espécie listada na base de dados, após uma cautelosa limpeza dos registros. Ao final da compilação dos dados geramos uma base de dados com cerca de 300.000 registros georeferenciados. Esses pontos 
foram cruzados com uma grade de hexágonos de área igual a um milhão de hectares, que também foi classificada de acordo com o bioma da sua localização. Para cada espécie calculamos a proporção de hexágonos presentes em cada bioma, sendo desprezados os biomas com menos de $20 \%$ de ocorrência da espécie. Para as espécies com ocorrência predominante no Cerrado, conferimos seu bioma de distribuição de acordo com a Lista de Espécies da Flora do Brasil (JBRJ, 2014). Para verificarmos a localização predominante das espécies compartilhadas entre biomas, plotamos os mapas dos hexagons da riqueza observada para essas espécies. Para identificar as lacunas de amostragem e direcionar estudos posteriores, geramos um mapa com o número de estudos existentes em cada hexágono de um milhão de hectare. Todos os procedimentos para processamento de mapas e análises espaciais foram realizados com o programa ArcGIS 10 (ESRI 2011).

\section{RESULTADOS}

Foram compilados 167 estudos (Tabela S1), referentes a 625 localidades, para as quais foram identificadas 909 espécies arbóreas de cerrado (Tabela S2), distribuídas em 298 gêneros e 76 famílias. As famílias mais representativas em termos de número de espécies são Fabaceae $(n=146)$, Myrtaceae $(n=84)$, Melastomataceae $(n=46)$, Lauracea $(n=39)$ e Rubiaceae $(n=37)$. As com maior número de gêneros são Fabaceae $(n=58)$ e Rubiaceae $(n=23)$. São também comuns as famílias com apenas um gênero (30 famílias). Em termos de frequência, as famílias Fabaceae, Vochysiaceae, Malpiguiaceae, Bignoniaceae, Apocynaceae, Myrtaceae Rubiaceae e Annonaceae ocorreram em mais de $80 \%$ das localidades, nessa ordem de importância.

Os gêneros mais especiosos são Miconia $(n=27)$, Myrcia $(n=23)$, Eugenia $(n=22)$ e Byrsonima $(n=19)$. Cento e cinquenta gêneros são representados por apenas uma espécie. Das 909 espécies registradas, 249 são unicatas e apenas 154 ocorrem em mais de 10\% das localidades. As espécies mais frequentes, que ocorrem entre 65 e $85 \%$ das localidades, são Qualea grandiflora, Qualea parvifolia, Bowdichia virgilioides, Hymenaea stigonocarpa, Connarus suberosus, Byrsonima coccolobifolia, Dimorphandra mollis e Handroanthus ochracius. As espécies dominantes, seguindo o critério sugerido por Bridgewater et al. (2004) (de ocorrência em mais de $20 \%$ das localidades) somam 100 espécies (Tabela S1).

O número de espécies arbóreas registradas por levantamento variou de 5 a 158 (60 $\pm 25)$, e de espécies predominantemente do Cerrado está entre 0 e $60(24 \pm 12)$. Já o número 
médio de famílias registradas por localidade foi $28( \pm 8)$, sendo que o mínimo registrado foram quatro e o máximo a 52 famílias em uma localidade no Tocantins (Figura 1). Os hexágonos mais distantes do centroide do bioma possuem maior número cumulativo de espécies, e a terceira classe de distância em relação ao centroide possui menor riqueza acumulada (Figura 2).

Em relação ao compartilhamento de espécies entre o cerrado e os biomas adjacentes, 15\% são de ampla distribuição, ou seja, ocorrem em alta frequência em mais de dois biomas (Figura 3). A maior parte das espécies do cerrado é compartilhada com a Mata Atlântica (44\%), seguida da Amazônia (15\%), da Caatinga (9\%) e do Pantanal (1\%). A proporção de espécies predominantemente do cerrado é de $16 \%$ do total. Quando conferimos a distribuição dessas espécies pela Lista de Espécies da Flora do Brasil, apenas 47 (5\%) são consideradas restritas ao Cerrado. A riqueza total do cerrado foi estimada entre 1000 e 1308 espécies arbóreas (Tabela 1). A maioria dos hexágonos não inventariados está nas regiões de ecótono entre o Cerrado e os biomas adjacentes (Figura 4).

\section{DISCUSSÃO}

As maiores riquezas absolutas estão em localidades centrais do Cerrado, sugerindo um padrão afetado pelo efeito do domínio médio (Colwell \& Hurtt 1994), ou seja, a sobreposição das distribuições das espécies soma um alto número de espécies na região central do Cerrado. Esse padrão também tem sido registrado para outros grupos taxonômicos, como os anfíbios, répteis, aves e mamíferos (Diniz-Filho et al. 2009). No entanto, nas regiões de transição entre os biomas há grande oportunidade para surgirem espécies acessórias (Rizzini 1963), conferindo alta diversidade beta aos ecótonos, como observado entre a Caatinga e o Cerrado (Castro et al. 1998). Esses resultados são de extrema importância para direcionar políticas de conservação e novas prospecções biológicas, já que essas zonas de transição merecem redobrada atenção frente às mudanças climáticas, pois as populações das plantas dessas regiões podem possuir alta plasticidade fisiológica. Novas investigações nesse sentido poderão trazer importantes informações para subsidiar a conservação da biodiversidade. Mesmo incluindo baixa riqueza local, regiões de ecótonos são parte importante da biota do Cerrado. As regiões de ecótono, no entanto, possuem menor número de estudos por hexágono, e muitas vezes esses hexágonos sequer foram inventariados. 
A localidade com maior riqueza apresentou 158 espécies, o que é um valor alto em comparação com as demais localidades, sendo raro observar mais de 120 espécies por localidade (Ratter \& Dargie 1992). Esse número de espécies foi registrado em poucas ocasiões em inventários de um hectare (Haidar, Dias, \& Felfili, 2013; Oliveira-Filho \& Ratter, 2002) ou por levantamentos florísticos (Batalha \& Martins 2007; Ratter et al. 2011). O alto turnover de espécies entre as localidades já foi registrado diversas vezes para o Cerrado (Bridgewater et al. 2004; Felfili \& Silva-Júnior 2005; Felfili et al. 1994, 2001, 2004; Felfili; Sousa-Silva; et al. 2005; Felfili \& Felfili 2001; Oliveira-Filho \& Ratter 2002), e nesse caso não foi diferente, já que apenas 154 espécies ocorreram em mais de 10\% das localidades. As 249 unicatas representam uma importante parcela da riqueza conhecida para o bioma, no entanto a maior parte dessas espécies possui distribuição predominante em outros biomas, com ocorrência marginal no Cerrado, como descrito anteriormente para os cerrados dos estados do Piauí e Maranhão (Heringer et al. 1977).

Em estudo de 376 localidades, Bridgewater et al. (2004) observaram 951 espécies de árvores e arbustos lenhosos. O presente estudo teve como ponto de partida a mesma base de dados, no entanto, foram mantidas apenas as espécies arbóreas, o que corresponde a 746 espécies do estudo de Bridgewater et al. (2004). Com a compilação de outros estudos, incluímos 139 espécies de árvores a essa base de dados, tendo previsão de acréscimo de pelo menos outras 93 espécies.

A proporção de espécies arbóreas e arbustivas por localidade no cerrado é cerca de 1:3 (Batalha \& Martins 2007), e a proporção do número de gêneros foi estimada em 1:2,5 (Rizzini 1963). A fim de suprir essa lacuna, sugerimos a inclusão de levantamento de espécies lenhosas de menores diâmetros nos inventários e levantamentos sistemáticos da vegetação. O manual de parcelas permanentes do bioma Cerrado (Felfili; Carvalho; et al. 2005) tem norteado os estudos fitossociológicos e inventários do bioma, com foco em espécies arbóreas. Mesmo nas subparcelas de regeneração são incluídas apenas as espécies arbóreas de pequeno porte. A inclusão de espécies arbustivas nessas subparcelas de regeneração já representaria um grande avanço para melhoria do entendimento da biodiversidade do Cerrado. Isso porque apenas $15 \%$ das espécies arbóreas registradas para o cerrado sentido amplo tem distribuição predominante no Cerrado, quando na realidade proporção muito maior é registrada no estrato arbustivo 
(Mendonça et al. 2008). Além disso, diversas espécies encontradas com porte arbóreo em algumas localidades, não passam do estrato arbustivo em outras (Ferri \& Coutinho 1958).

Altas taxas de endemismo e grande ameaça à biodiversidade são os dois critérios utilizados para a definição dos hotspots de biodiversidade (Myers et al. 2000). Esse status é extremamente valioso para o Cerrado, cujas taxas de desmatamento atingiram níveis alarmantes nas últimas décadas (IBAMA 2009, 2011; Klink \& Machado 2005). No entanto, a proporção de árvores endêmicas contabilizado com a presente base de dados foi de apenas $5 \%$. Outras 71 árvores são consideradas endêmicas do Cerrado na Lista de Espécies da Flora do Brasil. Algumas dessas espécies não foram registradas na presente base de dados. Outras foram amplamente registradas em outros biomas, quando observamos os registros que obtivemos no repositório SpeciesLink, o que nos levou a não considera-las endêmicas. Assim, para compreendermos os padrões de distribuição da biodiversidade do bioma Cerrado, é de extrema importância novos estudos abrangendo espécies arbustivas e herbáceas, estratos que devem conter maior proporção de endemismo dos $44 \%$ estimados para as plantas do Cerrado (Myers et al. 2000).

A proporção de espécies compartilhadas com a Mata Atlântica é três vezes maior do que as compartilhadas com a Amazônia, apesar da maior fronteira do Cerrado ser com a Amazônia. Esse mesmo padrão foi descrito por Rizzini (1963) e por Heringer et al., (1977), que quantificaram o número de gêneros encontrados nos demais biomas brasileiros, mostrando maior proporção compartilhada com a Mata Atlântica (80\%) do que com a Amazônia (77\%) (Heringer et al. 1977). Entretanto novos levantamentos nas grandes lacunas de informação existentes na Amazônia (Sousa-Baena et al. 2014) certamente poderão diminuir essa proporção. Tendo em vista a baixa amostragem na Amazônia, também não foi possível identificar um padrão de distribuição da riqueza das espécies compartilhadas entre o Cerrado e a Amazônia (Figura 5D).

O maior número de espécies compartilhadas entre o Cerrado e a Caatinga está localizado na transição entre os biomas (Figura 5B), não sendo adequado afirmar que essas espécies são "acessórias", pois aparentemente essas espécies são igualmente importantes para a composição florística dos dois biomas. A região com maior presença dessas espécies é a parte norte da Cadeia do Espinhaço, região com muitos encraves de cerrado em meio à Caatinga. $O$ termo "espécie acessória" usado por Rizzini (1963) e por Heringer et al., (1977) é artificial em 
termos ecológicos (Castro et al. 1999), já que não se sabe o bioma de origem dessas espécies. Além disso, a ocorrência da espécie em uma área faz dela parte da biota local (Castro et al. 1999).

As espécies compartilhadas entre o Cerrado e a Mata Atlântica estão localizadas principalmente no Cerrado da região do estado de São Paulo, na parte sul da Cadeia do Espinhaço e nas adjacências dessas duas (Figura 5C). O Cerrado de São Paulo compõe a região biogeográfica Sul (Capítulo 2 dessa tese), que tem influência importante da Mata Atlântica. $\mathrm{Na}$ Cadeia do Espinhaço há predomínio dos campos de altitude, uma vegetação de áreas abertas, em condições climáticas muito diferenciadas das florestas ombrófilas. Vários desses campos de altitude fazem fronteira com o Cerrado, e por apresentar condições climáticas parecidas determinadas pela altitude, é esperado grande número de espécies em comum. Com isso, mais uma vez observamos que as espécies compartilhadas são igualmente importantes para os dois biomas.

Comparando o presente estudo e os anteriores (Bridgewater et al. 2004; Castro et al. 1999; Heringer et al. 1977; Rizzini 1963), incluindo estudos locais (Batalha \& Martins 2007) em relação ao número de espécie por famílias no cerrado, as mesmas famílias aparecem como mais numerosas, mas não necessariamente na mesma ordem. Os gêneros mais numerosos apresentados aqui (Miconia, Myrcia, Eugenia e Byrsonima) apresentaram grande variação no número de espécies quando comparado com a compilação feita por Heringer et al. (1977), onde Byrsonima foi o gênero mais especioso $(n=22)$, seguido por Myrcia $(n=18)$, Kielmeyera $(n=16)$ e Miconia $(n=15)$ e Annona $(n=11)$. As diferenças são ainda maiores quando comparamos com os dados mostrados por Rizzini (1963), onde os gêneros mais representativos foram Miconia e Bombax ( $n=11)$, Byrsonima ( $n=10)$, Annona e Aspidosperma $(n=9)$ e Cassia, Myrcia e Qualea $(\mathrm{n}=8)$.

Considerando todas as espécies encontradas no cerrado, a família Vochysiaceae não apresenta os maiores números de espécies. No entanto, quando consideradas apenas as espécies com distribuição predominante no cerrado, a família Vochysiaceae é a segunda em número de espécies, assim como observado por Rizzini (1963). Além disso, essa família está entre as a mais frequentes no Cerrado, pela ampla distribuição de Qualea parviflora, $Q$. grandiflora e Q. multiflora (Oliveira-Filho \& Ratter 2002). Nossas observações não foram diferentes, já que essas espécies estão entre as de maiores frequência. 
Comparando parâmetros ecológicos de cerrado sentido restrito em três localidades, Ferri \& Coutinho (1958) sugeriram que há uma uniformidade nessa fitofisionomia, dada pela presença marcante de espécies amplamente distribuídas. Cento em vinte espécies são consideradas as espécies cujas presenças caracterizam a ocorrência do bioma (Bridgewater et al. 2004; Ratter \& Dargie 1992; Ratter et al. 1996, 2003). Usando os mesmos parâmetros para revisar as espécies dominantes do Cerrado, nós encontramos 20 espécies a menos (Bridgewater et al. 2004).

Algumas famílias apresentaram baixo número de espécies, sendo que 12 das 76 apresentaram apenas uma espécie cada. Essa característica da vegetação do cerrado é uma das razões pelas quais se atribui alta proporção de endemismo dessa vegetação arbórea (Batalha \& Martins 2007). No entanto, essa não é uma razão aceitável, já que essas famílias em sua maioria são características de outros biomas ou outras fitofisionomias, ocorrendo ocasionalmente no cerrado. As famílias e gêneros com apenas uma espécie devem ter distribuição relictual, com baixa diversificação na região do Brasil central, ou uma nova colonização, o que precisa ser averiguado em estudos posteriores.

Observamos alta riqueza e diversidade beta para as árvores do Cerrado, assim como grande número de famílias e gêneros, sendo muitos com apenas uma espécie. A maioria das espécies do Cerrado ocorrem também na Mata Atlântica, e o número de espécies endêmicas observado foi menor do que o estimado para a flora do Cerrado. Possivelmente a maior proporção de espécies endêmicas é encontrada em outras formas de vida, como ervas, lianas, arbustos e suarbustos, sendo importante que essas espécies sejam incluídas nos inventários e lenvantamentos florísticos. Para subsidiar novos estudos e complementar o conhecimento sobre a flora do Cerrado, identificamos as principais lacunas de amostragem, localizadas em sua maioria nas regiões de transição entre os biomas. Essas regiões de transição são responsáveis pela maior parte das espécies registradas nos estudos do Cerrado.

\section{AGRADECIMENTOS}

À CAPES, CNPq e Programa Ciência Sem Fronteira pelas bolsas de estudos concedidas a RDF. Ao CNPq pela bolsa de produtividade em pesquisa concedida a RBM. À Fundação O Boticário e ao Centro Agroecológico do Cerrado pelo apoio aos trabalhos em campo. À Rede ComCerrado pelo fornecimento da estrutura de laboratório. 


\section{REFERÊNCIAS}

Batalha MA \& Martins FR, 2007. The vascular flora of the cerrado in Emas National Park (Central Brazil): a savanna flora summarized. Brazilian Archives of Biology and Technology, 50(2):269-277.

Boulinier T, Nichols JD, Sauer JR, Hines JE \& Pollock KH, 1998. Estimating species richness: the importance of heterogeneity in species detectability. Ecology, 79(3):1018-1028.

Bridgewater S, Ratter JA \& Ribeiro JF, 2004. Biogeographic patterns, Beta-diversity and dominance in the cerrado biome of Brazil. Biodiversity and Conservation, 13(12):22952318.

Castro AAJF, Martins FR \& Fernandes AG, 1998. The woody flora of cerrado vegetation in the state of Piaui, northeastern Brazil. Edinburgh Journal of Botany, 55(03):455-472.

Castro AAJF, Martins FR, Tamashiro JY \& Shepherd GJ, 1999. How rich is the flora of Brazilian Cerrados? Annals of the Missouri Botanical Garden, 86(1):192-224.

Clements FE \& Shelford V, 1939. Bio-ecology. New York, USA / London, UK: John Wiley \& Sons / Chapman \& Hall, p. 425.

Colwell RK \& Hurtt GC, 1994. Nonbiological Gradients in Species Richness and a Spurious Rapoport Effect. The American naturalist, 144(4):570-595.

Coutinho LM, 2006. O conceito de bioma. Acta Botanica Brasilica, 20(1):13-23.

Diniz-Filho JAF, Bini LM, Oliveira G De, et al., 2009. Macroecologia, biogeografia e áreas prioritárias para conservação no cerrado. Oecologia Brasiliensis, 13(03):470-497.

Eiten G, 1972. The Cerrado vegetation of Brazil. The Botanical Review, 38(2):201-341.

ESRI, 2011. ArcGIS Desktop. Redlands, CA: Environmental Systems Research Institute,

Felfili JM, Carvalho FA \& Haidar RF, 2005. Manual para o monitoramento de parcelas permanentes nos biomas Cerrado e Pantanal. Brasília, DF: Universidade de Brasília, Departamento de Engenharia Florestal.

Felfili JM, Sevilha AC \& Silva-Júnior MC, 2001. Comparação entre as unidades fisiográficas Chapada Pratinha, Veadeiros e Espigão Mestre do São Francisco. In JM Felfili \& MC Da Silva-Júnior (eds.), Biogeografia do Bioma Cerrado: estudo fitofisionomico na Chapada do Espigão Mestre do São Francisco. 1st ed., Brasília, DF: Universidade de Brasília, Departamento de Engenharia Florestal, p. 152.

Felfili JM \& Silva-Júnior MC, 2005. Diversidade alfa e beta no cerrado sensu stricto, Distrito Federal, Goiás, Minas Gerais e Bahia. In A. SCARIOT JC. SOUSA-SILVA \& J. FELFILI (eds.), Cerrado: ecologia, biodiversidade e conservação. Brasília, DF: Ministério do Meio Ambiente, p. 141-154. 
Felfili JM, Silva-Júnior MC, Filgueiras TS, et al., 1994. Projeto biogeografia do bioma cerrado: vegetação e solos. Cadernos de Geociências, 12(4):75-167.

Felfili JM, Silva-Júnior MC, Sevilha AC, et al., 2004. Diversity, floristic and structural patterns of cerrado vegetation in Central Brazil. Plant Ecology, 175(1):37-46.

Felfili JM, Sousa-Silva JC \& Scariot A, 2005. Biodiversidade, ecologia e conservação do Cerrado: avanços no conhecimento. In A Scariot JC Sousa-Silva \& JM Felfili (eds.), Cerrado: ecologia, biodiversidade e conservação. Brasília: Ministério do Meio Ambiente, p. 25-44.

Felfili MC \& Felfili JM, 2001. Diversidade alfa e beta no cerrado sensu stricto da Chapada Pratinha, Brasil. Acta Botanica Brasilica, 15(2):243-254.

Ferri MG \& Coutinho LM, 1958. Contribuição ao conhecimento da ecologia do cerrado. Estudo comparativo da economia d'água de sua vegetacao, em Emas (Est. de São Paulo), Campo Grande (Est. de Mato Grosso) e Goiânia (Est. de Goiás). Boletim da Faculdade de Filosofia, Ciências e Letras, Universidade de São Paulo. Botânica, 12:103-150.

Forni-Martins ER \& Martins FR, 2000. Chromosome studies on Brazilian cerrado plants. Genetics and Molecular Biology, 23(4):947-955.

Haidar RF, Dias R \& Felfili JM, 2013. Mapeamento das regiões fitoecológicas e inventário florestal do estado do Tocantins. Palmas, TO: Governo do Estado do Tocantins, p. 156.

Heringer EP, Barroso GM, Rizzo JA \& Rizzini CT, 1977. A flora do Cerrado. In MG Ferri (ed.), IV Simpósio sobre o Cerrado. São Paulo, SP: Editora da Universidade de São Paulo, p. 211232.

Hoffmann WA \& Silva-Júnior ER, 2005. Seasonal leaf dynamics across a tree density gradient in a Brazilian savanna. Functional Ecology, 19(6):932-940.

IBAMA, 2009. Relatório técnico de monitoramento do desmatamento no bioma Cerrado, 2002 a 2008: dados revisados. Brasília, DF: IBAMA,

IBAMA, 2011. Monitoramento do desmatamento nos biomas brasileiros por satélite. Brasília, DF: IBAMA,

JBRJ. Lista de Espécies da Flora do Brasil. (http://floradobrasil.jbrj.gov.br/).

Klink CA \& Machado RB, 2005. Conservation of the Brazilian Cerrado. Conservation Biology, 19(3):707-713.

Lomolino M V., Riddle BR \& Brown JH, 2006. Biogeography. 3rd ed., Sunderland: Sinauer Associates.

Machado RB, Aguiar LMS, Castro AAJF, Nogueira CC \& Ramos-Neto MB, 2008. Caracterização da fauna e flora do Cerrado. In , Savanas: desafios e estratégias para o equilíbrio entre sociedade, agronegócio e recursos naturais. EMBRAPA ed., Brasília, DF, p. 284-300. 
Méio BB, Freitas C V., Jatobá L, et al., 2003. Influência da flora das florestas Amazônica e Atlântica na vegetação do cerrado sensu stricto. Revista Brasileira de Botânica, 26(4):437444.

Mendonça RC, Felfili JM, Walter BMT, et al., 2008. Flora vascular do bioma Cerrado: checklist com 12.356 espécies. In S Sano S Almeida \& J Ribeiro (eds.), Cerrado: ecologia e flora. Brasília, DF: Embrapa Informação Tecnológica, p. 423-1279.

Myers N, Mittermier RA, Mittermier CG, et al., 2000. Biodiversity hotspots for conservation priorities. Nature, 403(24):853-858.

Noss RF, 1996. Ecosystems as conservation targets. Trends in Ecology \& Evolution, 11(8):351.

Oliveira-Filho AT \& Ratter JA, 1995. A study of the origin of central Brazilian forests by the analysis of plant species distribution patterns. Edinburgh Journal of Botany, 52(02):141.

Oliveira-Filho AT \& Ratter JA, 2002. Vegetation Physiognomies and Woody Flora of the Cerrado Biome. In PS Oliveira \& RJ Marquis (eds.), The Cerrados of Brazil: ecology and natural history of a neotropical savanna. New York: Columbia University Press, p. 93-120.

Ratter JA, Bridgewater S, Atkinson R \& Ribeiro JF, 1996. Analysis of the floristic composition of the Brazilian cerrado vegetation II: Comparison of the woody vegetation of 98 areas. Edinburgh Journal of Botany, 53(02):153.

Ratter JA, Bridgewater S \& Ribeiro JF, 2003. Analysis of the Floristic Composition of the Brazilian Cerrado Vegetation III: Comparison of the Woody Vegetation of 376 Areas. Edinburgh Journal of Botany, 60(01):57-109.

Ratter JA, Bridgewater S, Ribeiro JF, et al. Analysis of the floristic composition of the Brazilian cerrado vegetation IV: Presentation of a Revised Data-Base of 367 Areas. (http://cerrado.rbge.org.uk/cerrado/download/download.php).

Ratter JA \& Dargie TCD, 1992. An analysis of the floristic composition of 26 cerrado ares in Brazil. Edinburgh Journal of Botany, 49(02):235.

Ribeiro JF \& Walter BMT, 1998. Fitofisionomias do Bioma Cerrado. In SM Sano \& SP Almeida (eds.), Cerrado: ambiente e flora. Planaltina-DF: EMBRAPA-CPAC.

Ribeiro JF \& Walter BMT, 2008. As principais fitofisionomias do bioma Cerrado. In SM Sano SP Almeida \& JF Ribeiro (eds.), Cerrado: ecologia e flora. Brasília, DF: EMBRAPA, p. 151-212.

Rizzini CT, 1963. A flora do Cerrado. In , Simpósio sobre o Cerrado. São Paulo, SP: Editora da Universidade de São Paulo.

Silva JMC \& Bates JM, 2002. Biogeographic Patterns and Conservation in the South American Cerrado: A Tropical Savanna Hotspot. BioScience, 52(3):225-234.

Silva LCR, Haridasan M, Hoffmann W a., Sternberg LSL \& Franco AC, 2010. Not all forests are expanding over central Brazilian savannas. Plant and Soil, 333(1-2):431-442. 
Silva LCR, Sternberg L, Haridasan M, et al., 2008. Expansion of gallery forests into central Brazilian savannas. Global Change Biology, 14(9):2108-2118.

530

Sousa-Baena MS, Garcia LC \& Peterson AT, 2014. knowledge of the plants of Brazil and. Diversity and Distributions, 20(4):369-381.

532 Walter BMT \& Guarino EDSG, 2006. Comparação do método de parcelas com o "levantamento rápido" para amostragem da vegetação arbórea do Cerrado sentido restrito. Acta Botanica Brasilica, 20(2):285-297. 

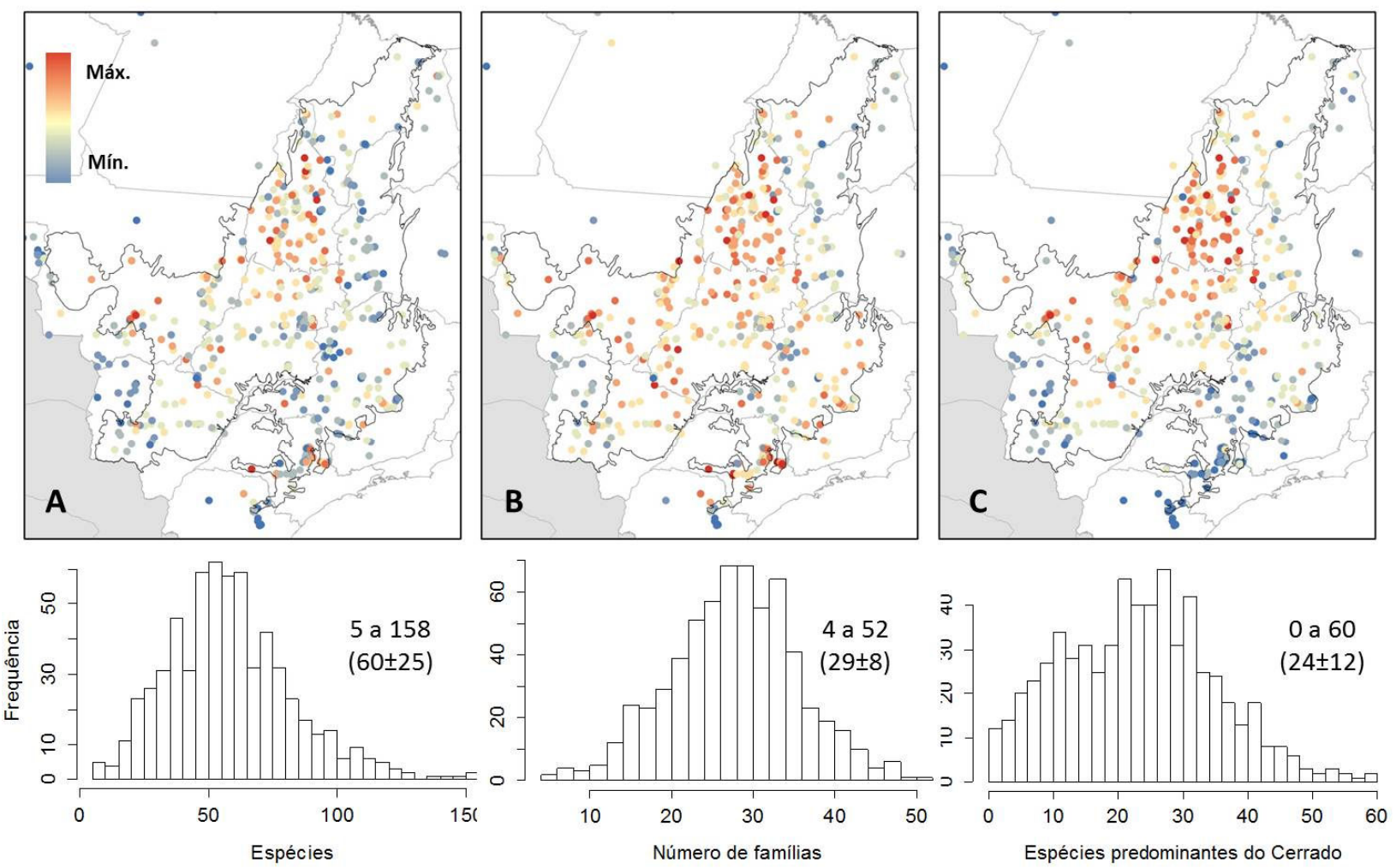

540

541 Figura 1. Frequência, mínimo, máximo, média e desvio padrão de (A) espécies; (B) famílias; e (C) 542 espécies predominantes no Cerrado nas localidades dos inventários e levantamentos florísticos 543 no Cerrado. 

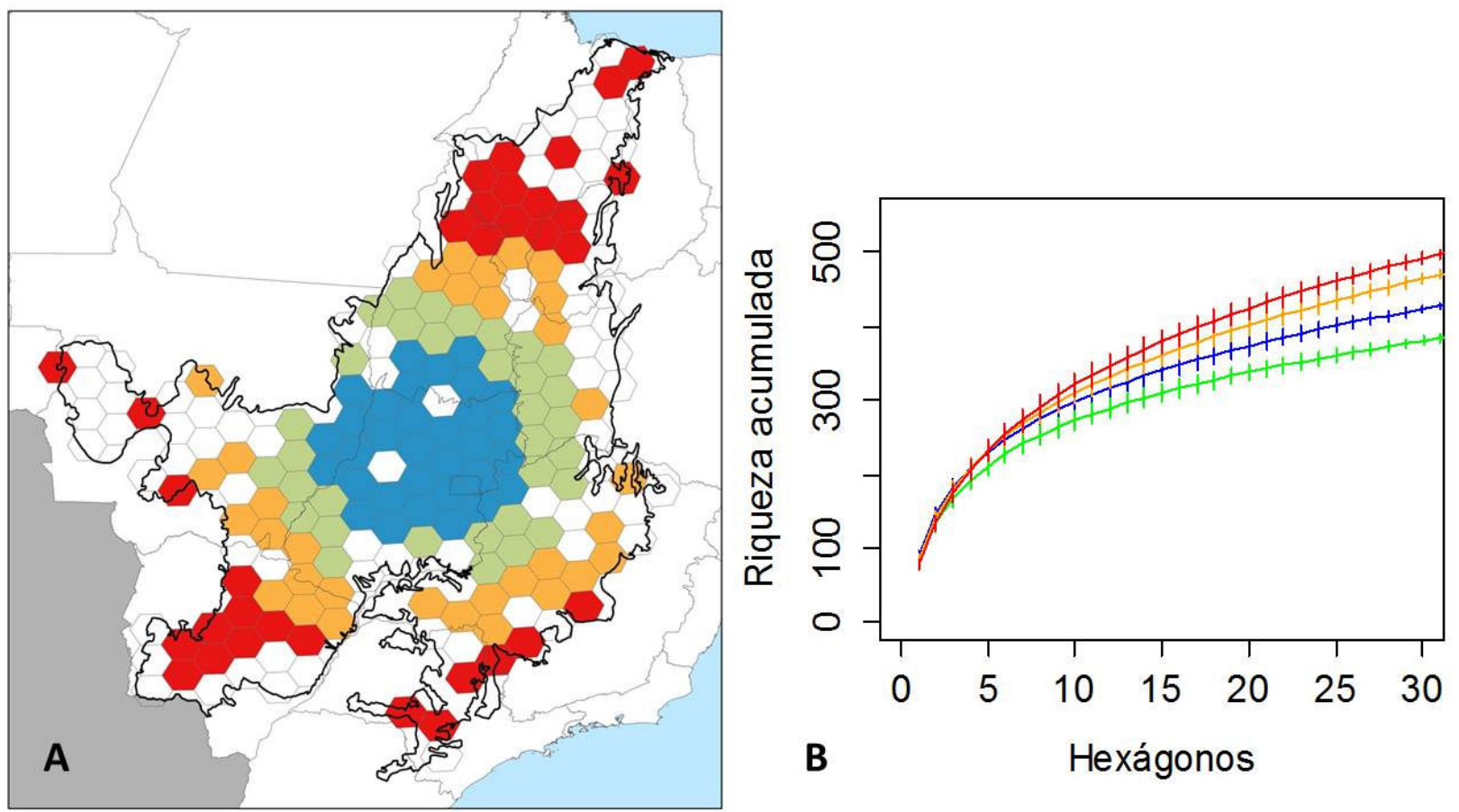

546 Figura 2. (A) Quatro classes de distância ao centroide do bioma e; (B) curvas de rarefação

547 dessas classes de distância. Os hexágonos mais distantes do centro do bioma possuem maior 548 riqueza acumulada de espécies. 


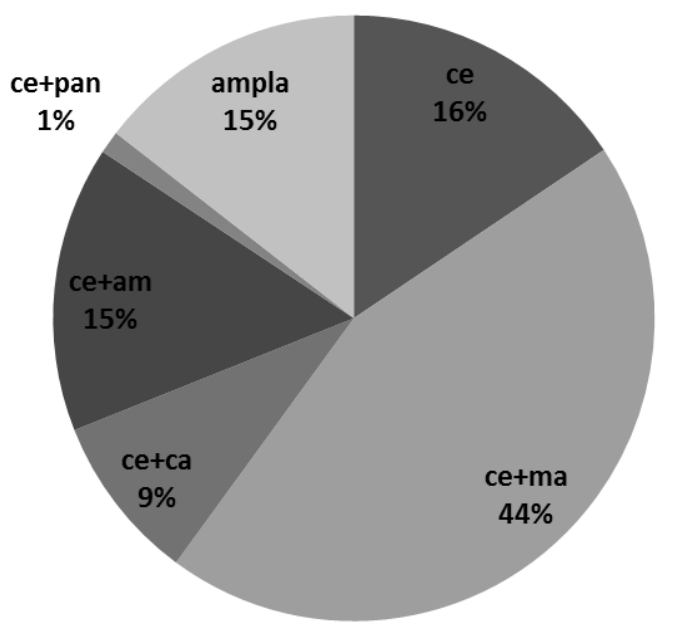

550

551 Figura 3. Proporção de espécies compartilhadas entre o Cerrado e os biomas adjacentes, dentre

552 as registradas em 625 levantamentos florísticos de cerrado sentido amplo. Os biomas 553 analisados foram Amazônia (am), Caatinga (ca), Cerrado (ce), Mata Atlântica (ma), Pampa 554 (pam) e Pantanal (pan). A espécie foi considerada de ampla distribuição quando ocorreu em 555 pelo menos três biomas.

556 


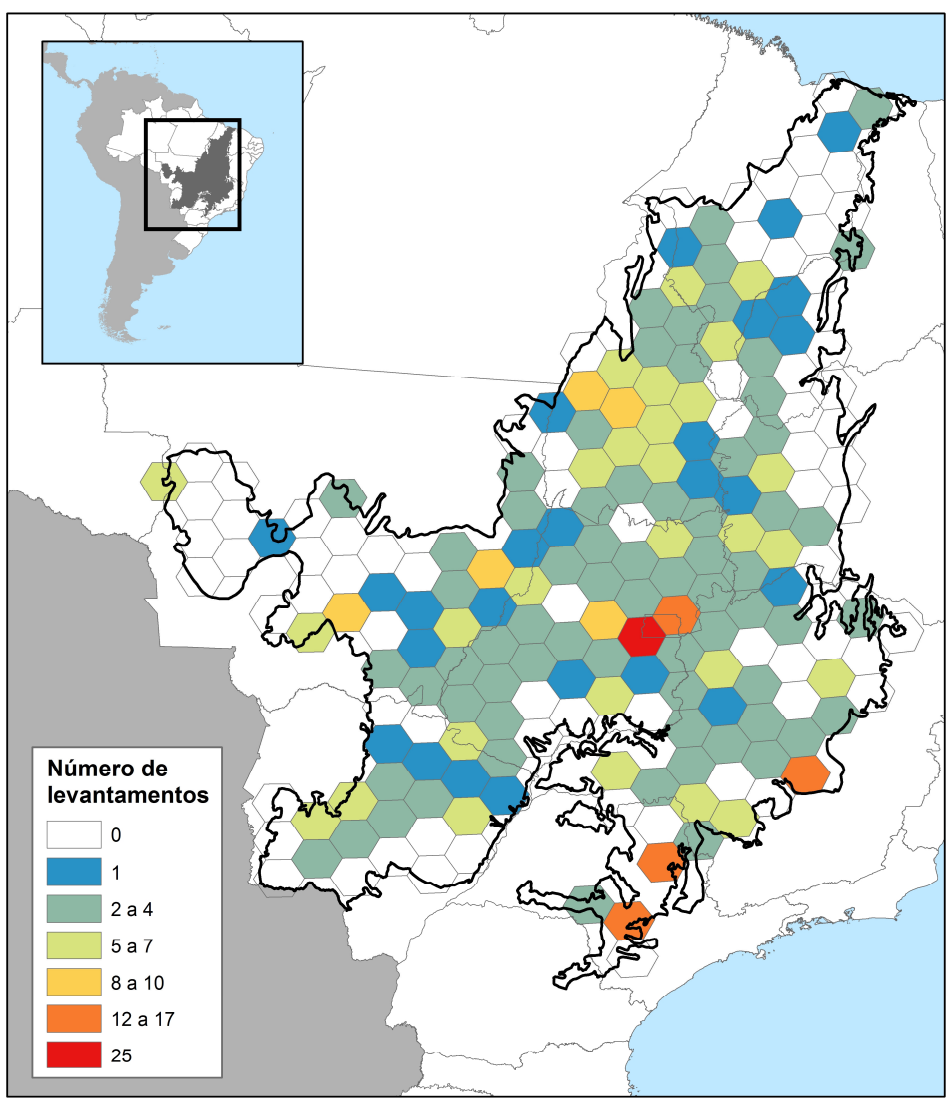

559 Figura 4. Número De levantamentos florísticos e fitossociológicos em cerrado sentido amplo 560 (Ribeiro e Walter, 200x) no bioma Cerrado. 

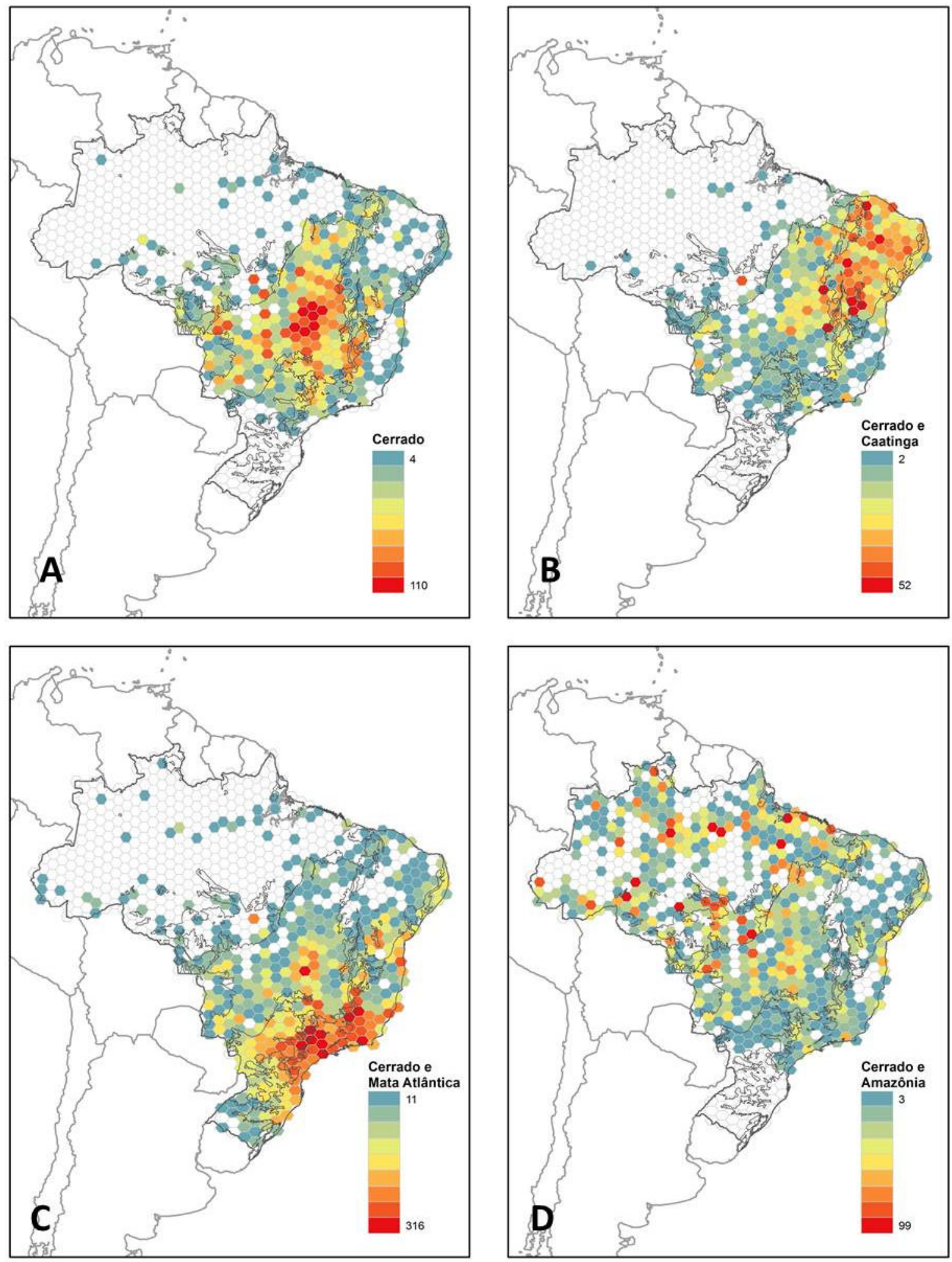

Figura 5. Número de espécies por hexágono de 1.000.000 ha para (A) espécies predominantes do Cerrado, (B) espécies compartilhadas principalmente entre o Cerrado e a Caatinga, (C) espécies compartilhadas principalmente entre o Cerrado e a Mata Atlântica, (D) espécies compartilhadas principalmente entre o Cerrado e a Amazônia. 
570 Tabela 1. Estimativa de riqueza de espécies e de número de espécies predominantemente do 571 Cerrado usando quatro diferentes estimadores. A riqueza observada é proveniente de 572 compilação de 576 inventários e levantamentos florísticos em cerrado sentido amplo no bioma 573 Cerrado e em encraves dessa fitofisionomia em outros biomas.

\begin{tabular}{|c|c|c|c|c|c|c|c|}
\hline & $\begin{array}{c}\text { Riqueza } \\
\text { obs. }\end{array}$ & Chao I & Jackknife1 & $\begin{array}{c}\text { Jackknife } \\
2\end{array}$ & Bootstrap & $\begin{array}{c}\text { Mínim } \\
\text { o }\end{array}$ & Máximo \\
\hline Total & 910 & $1223 \pm 53$ & $1159 \pm 32$ & 1308 & $1019 \pm 19$ & 1000 & 1308 \\
\hline
\end{tabular}

574

575

576 
578 Estudos compilados para a elaboração da base de dados de espécies arbóreas de cerrado 579 sentido amplo.

\section{Referência base de dados}

Abreu MF, Pinto JRR, Maracahipes L, et al., 2012. Influence of edaphic variables on the floristic composition and structure of the tree-shrub vegetation in typical and rocky outcorp cerrado areas in Serra Negra, Goias State, Brazil. Brazilian Journal of Botany, 35(3):259-272.

Amaral AG, Pereira FFO \& Munhoz CBR, 2006. Fitossociologia de uma área de cerrado rupestre na fazenda Sucupira, Brasília-DF. Cerne, 12(4):350-359.

Amorim PK \& Batalha MA, 2007. Soil-vegetation relationships in hyperseasonal cerrado, seasonal cerrado, and wet grassland in Emas National Park (central Brazil). Acta Oecologica, 32(3):319327.

Andrade L \& Miranda HS, 2002. Effects of fire on the soil seed bank in a cerrado sensu stricto in central Brazil. In DX Viegas (ed.), IV International Conference on Forest Fire Research \& Wildland Fire Safety. IV International Conference on Forest Fire Research \& Wildland Fire SafetyCoimbra, Portugal: Millpress,

Antunes EM, Maieski A, Caxambu MG \& Mello J de F, 2009. Levantamento Florístico da estação ecológica do cerrado de Campo Mourão-PR. In Universidade de São Carlos (ed.), VI Congresso de Meio Ambiente da AUGM. VI Congresso de Meio Ambiente da AUGMSão Carlos, SP,

Aoki C, Pereira SR \& Matos WH, 2006. Florística e fitossociologia de um fragmento de cerrado em Itirapina-SP. In FAM. Santos F. Martins \& JY Tamashiro (eds.), Relatórios da disciplina NE211. . Campinas, SP: PPG-Ecologia, IB, Unicamp, p. 77-90.

Aquino FDEG, Machado B, Walter T, Ribeiro JF \& Walter BMT, 2007. Woody community dynamics in two fragments of "cerrado" stricto sensu over a seven-year period (1995-2002), MA, Brazil. Revista Brasileira de Botânica, 30(1):111-119.

Assunção SL \& Felfili JM, 2004. Fitossociologia de um fragmento de cerrado sensu stricto na APA do Paranoá, DF, Brasil. Acta Botanica Brasilica, 18(4):903-909.

Balduíno AP do C, Souza AL De, Meira-Neto JAA, Silva AF Da \& Silva-Júnior MC, 2005. Fitossociologia e análise comparativa da composição florística do cerrado da flora de Paraopeba-MG. Revista Árvore, 29(1):25-34.

Barbosa MM, 2006. Florística e fitossociologia de cerrado sentido restrito no parque estadual da Serra Azul, Barra do Garça. Dissertação de Mestrado. PPG Ciencias Biológicas. Universidade Federal de Mato Grosso.

Barbosa RI, Nascimento SP Do, Amorim PAF De \& Silva RF Da, 2005. Notas sobre a composição arbóreo-arbustiva de uma fisionomia das savanas de Roraima, Amazônia Brasileira. Acta Botanica Brasilica, 19(2):323-329.

Barreira S, Botelho SA, Scolforo JR \& Mello JM De, 2000. Efeito de diferentes intensidades de corte seletivo sobre a regeraçãa natural de cerrado. Cerne, 6(1):040-051.

Batalha MA \& Mantovani W, 2001. Floristic composition of the cerrado in the pé-de gigante reserve (Santa Rita do Passa Quatro, southeastern Brazil). Acta Botanica Brasilica, 15(3):289-304.

Batalha MA, 2001. Florística, espectro biológico e padrões fenológicos do cerrado sensu lato no Parque Nacional das Emas (GO) e o componente herbáceo-subarbustivo da flora do cerrado sensu lato. Tese de Doutorado. Campinas, SP: PPG Ecologia, Universidade Estadual de Campinas.

Batista ML, 2007. Estrutura e diversidade da flora lenhosa no domínio de Cerrado em Carbonita, MG. Dissertação de Mestrado. Viçosa, MG: PPG Botanica, Universidade Federal de Viçosa. 
Borges HBN \& Shepherd GJ, 2005. Flora e estrutura do estrato lenhoso numa comunidade de Cerrado em Santo Antônio do Leverger, MT, Brasil. Revista Brasileira de Botânica, 28(1):61-74.

Borges HBN, 2000. Biologia reprodutiva e conservação do estrato lenhoso numa comunidade do cerrado. Tese de Doutorado. Campinas, SP: PPG Biologia Vegetal, Universidade Estadual de Campinas.

Bosquetti $L$ de $B, 2008$. Análise da estrutura da paisagem e fitofisionomias do parque estadual dos Pirineus, Goiás, Brasil. Tese de Doutorado. Piracicaba, SP: PPG Ecologia Aplicada. Universidade de São Paulo.

Botini T, 2008. Composição florística, estrutura da vegetação e atributos químicos de solo de área de mineração de ouro a céu aberto: estudo de caso do garimpo da lavrinha, Pontes e Lacerda-MT. Dissertação de Mestrado. Cáceres, MT: PPG Ciências Ambientais. Universidade do Estado do Mato Grosso.

Brando PM \& Durigan G, 2004. Changes in cerrado vegetation after disturbance by frost (São Paulo State, Brazil). Plant Ecology, 175(2):205-215.

Bruziguessi EP, 2006. Levantamento fitossociológico da vegetação arbustivo- arbórea em um cerrado rupestre no município de Alto Paraíso, GO. In DPP-UnB (ed.), XII Congresso de Iniciação Científica da UnB. XII Congresso de Iniciação Científica da UnB.Brasília, DF: Universidade de Brasília,

Bueno ML, Rafael D, Neves M, et al., 2013. Influence of edaphic factors on the floristic composition of an area of cerradão in the Brazilian central-west. Acta Botânica Brasílica, 27(2):445-455.

Campos ÉP De, Duarte TG, Neri AV, et al., 2006. Composição florística de um trecho de cerradão e cerrado sensu stricto e sua relação com o solo na floresta nacional (Flona) de Paraopeba, MG, Brasil. Revista Árvore, 30(3):471-479.

Cardoso E, Moreno MIC \& Guimarães AJM, 2002. Estudo fitossociológico em área de cerrado sensu stricto na estação de pesquisa e desenvolvimento ambiental Galheiros - Perdizes, MG. Caminhos de Geografia, 3(5):30-43.

Carvalho AR \& Marques-Alves S, 2008. Diversidade e índice sucessional de uma vegetação de cerrado sensu stricto na Universidade Estadual de Goiás-EUG, campus de Anápolis. Revista Árvore, 32(1):81-90.

Carvalho DA De \& Martins FR, 2009. Shrub and tree species composition in the cerrados of southwest Minas Gerais. Cerne, 15(2):142-154.

Carvalho DA De, 1987. Composição florística e estrutura de cerrados do sudoeste de Minas Gerais. Tese de Doutorado. Campinas, SP: Instituto de Ciências. Universidade Estadual de Campinas.

Carvalho MB, Ishara KL \& Maimoni-Rodella RCS, 2010. Vascular flora of a cerrado sensu stricto remmant in Pratânia, state of São Paulo, southeastern Brazil. Check list, 6(3):350-357.

Castro AAJF, 1987. Florística e fitossociologia de um cerrado marginal brasileiro, parque estadual de Vaçununga, Santa Rita do Passa Quatro-SP. Dissertação de Mestrado. Campinas, SP: PPG Ciências Biológicas. Universidade Estadual de Campinas.

Castro AAJF, Castro NMCF, Costa JM, et al., 2007. Cerrados Marginais do Nordeste e Ecótonos Associados. Revista Brasileira de Biociências, 5(1):273-275.

Cavassan O, 1990. Florística e fitossociologia da vegetação lenhosa em um hectare de cerrado no Parque Ecológico municipal de Bauru (SP). Tese de Doutorado. Campinas, SP: Instituto de Ciências. Universidade Estadual de Campinas.

Conceição GM \& Castro AAJF, 2009. Fitossociologia de uma área de cerrado marginal, Parque Estadual do Mirador, Mirador, Maranhão. Scientia Plena, 5(10):1-16.

Costa AAA \& Araújo GM De, 2001. Comparação da vegetação arbórea de cerradão e de cerrado na Reserva do Panga, Uberlândia, Minas Gerais. Acta Botanica Brasilica, 15(1):63-72.

Costa CP Da, Cunha CN Da \& Costa SC, 2010. Caracterização da flora e estrutura do estrato arbustivo-arbóreo de um cerrado no Pantanal de Poconé , MT. Biota Neotropica, 10(3):61-73. 
Costa FV Da, Oliveira KN, Nunes YRF, et al., 2010. Florística e estrutura da comunidade arbórea de duas áreas de cerrado sentido restrito no norte de Minas Gerais. Cerne, 16(3):267-281.

Costa IR Da, Araújo FS De \& Lima-Verde LW, 2004. Flora e aspectos auto-ecológicos de um encrave de cerrado na chapada do Araripe, Nordeste do Brasil. Acta Botanica Brasilica, 18(4):759-770.

Costa JM Da, 2005. Estudo fitossociológico e sócio-ambiental de uma área de cerrado com potencial meliófilo no município de Castelo do Piauí, Piauí, Brasil. Disertação de Mestrado. Teresina, PI: PPG em Desenvolvimento e Meio Ambiente. Universidade Federal do Piauí.

De EL \& Marimon BS, 2010. Levantamento florístico na fazenda Remanso, Nova Xavantina, MT. Nova Xavantina, MT: Universidade de Brasília \& Universidade Estadual de Mato Grosso,

Durigan G, Nishikawa DLL, Rocha E, et al., 2002. Caracterização de dois estratos da vegetaçao em uma área de cerrado no município de Brotas, SP, Brasil. Acta Botanica Brasilica, 16(3):251-262.

Faleiro W, 2007. Composição florística e estrutura da comunidade arbórea em duas áreas de cerrado sensu stricto, em Uberlândia - Minas Gerais. Dissertação de Mestrado. Uberlândia, MG: PPG Ecologia e Conservação de Recursos Naturais. Universidade Federal de Uberlândia.

Farias RRS De \& Castro AAJF, 2004. Fitossociologia de trechos da vegetação do Complexo de Campo Maior, Campo Maior, PI, Brasil. Acta Botanica Brasilica, 18(4):949-963.

Felfili JM \& Silva-Júnior MC, 2005. Diversidade alfa e beta no cerrado sensu stricto, Distrito Federal, Goiás, Minas Gerais e Bahia. In A. SCARIOT JC. SOUSA-SILVA \& J. FELFILI (eds.), Cerrado: ecologia, biodiversidade e conservação. . Brasília, DF: Ministério do Meio Ambiente, p. 141-154.

Felfili JM, Nogueira PE, Silva-Júnior MC, Marimon BS \& Delitti WBC, 2002. Composição florística e fitossociologia do cerrado sentido restrito no município de Água Boa - MT. Acta Botanica Brasilica, 16(1):103-112.

Felfili JM, Rezende A V., Silva-Júnior MC \& Silva MA, 2000. Changes in the floristic composition of cerrado sensu stricto in Brazil over a nine-year period. Journal of Tropical Ecology, 16:579-590.

Felfili JM, Silva-Júnior MC, Filgueiras TS, et al., 1994. Projeto biogeografia do bioma cerrado: vegetação e solos. Cadernos de Geociências, 12:75-167.

Ferreira FG, 2010. Diversidade beta na vegetação da bacia do Rio Paracatu - MG. Dissertação de Mestrado. Brasília, DF: PPG Ciências Florestais. Universidade de Brasília.

Ferreira FM \& Forza RC, 2009. Florística e caracterização da vegetação da Toca dos Urubus, Baependi, Minas Gerais, Brasil. Biota Neotropica, 9(4):131-148.

Ferreira JN, 2006. Padrões de estrutura e diversidade da vegetação lenhosa relacionados à heterogeneidade espacial de água no solo em cerrado do Brasil Central. Tese de Doutorado. Brasília, DF: PPG Ecologia. Universidade de Brasília.

Ferreira-Júnior WG, 2009. Análise de gradientes vegetacionais e pedogeomorfológicos em floresta, cerrado e campo no Pantanal matogrossense, Barão de Melgaço, Mato Grosso. Tese de Doutorado. Viçosa, MG: PPG Botânica. Universidade Federal de Viçosa.

Fidelis AT \& Godoy SAP De, 2003. Estrutura de um cerrado stricto sensu na gleba Cerrado Pé-DeGigante, Santa Rita do Passa Quatro, SP. Acta Botanica Brasilica, 17(4):531-539.

Fiedler NC, Isaac Nuno Carvalho de Azevedo, Rezende A V., et al., 2004. Efeito de incêncios florestais na estrutura e composição florística de uma área de cerrado sensu stricto na fazenda Água Limpa. Revista Árvore, 28(1):129-138.

Fina BG, 2009. Caracterização fitofisionômica da fazenda experimental da Universidade Estadual de Mato Grosso do Sul, Municíipio de Aquidauana-MS. Tese de Doutorado. Rio Claro, SP: PPG Ciências Biológicas. Universidade Estadual Paulista Julio de Mesquita Filho.

Finger Z, 2008. Fitossociologia de comunidades arbóreas em savanas do Brasil central. Tese de Doutorado. Santa Maria, RS: PPG Engenharia Florestal. Universidade Federal de Santa Maria.

Fonseca GAB \& Silva-Júnior MC, 2004. Fitossociologia e similaridade florística entre trechos de Cerrado sentido restrito em interflúvio e em vale no Jardim Botânico de Brasília , DF. Acta 
Botanica Brasilica, 18(1):19-29.

Franczak DD, 2006. Dinâmica da comunidade arbórea em um cerradão do parque do Bacaba, Nova Xavantina-MT. Monografia de Conclusão de Curso. Nova Xavantina, MT: Departamento de Ciências Biológicas. Universidade Estadual do Mato Grosso.

Franczak DD, 2009. Mudança na comunidade lenhosa de um cerradão e um cerrado stricto sensu no parque do Bacaba, Nova Xavantina - MT. Dissertação de Mestrado. Nova Xavantina, MT: PPG Ciências Florestais. Universidade Federal de Mato Grosso.

Giacomo RG, 2009. Fitossociologia, aporte de serapilheira, estoque de carbono e nitrogênio em diferentes formações vegetais na Estação Ecológica de Pirapitinga - MG. Dissertação de Mestrado. Seropédica, RJ: Instituto de Ciências. Universidade Federal Rural do Rio de Janeiro.

Giannotti E, 1988. Composição florística e estrutura fitossociológica da vegetação de cerrado e de transição entre cerrado e mata ciliar da estação experimental de Itirapina. Dissertação de Mestrado. Campinas, SP: Instituto de Biologia. Universidade Estadual de Campinas.

Gimenez VMM, 2005. Estudo da recomposição florística do componente arbustivo-arbóreo em áreas utilizadas para o plantio de exóticas em um cerrado de Luiz Antônio-SP. Dissertação de Mestrado. Ribeirão Preto, SP: PPG Biologia Comparada. Universidade de São Paulo.

Haidar RF, Dias R \& Felfili JM, 2013. Mapeamento das regiões fitoecológicas e inventário florestal do estado do Tocantins. Palmas, TO: Governo do Estado do Tocantins, p. 156.

Haidar RF, Françoso R, Carneiro D, et al., 2013. Estudo para criação e manejo de áreas protegidas no cerrado. Relat. Brasília, DF: Centro de Desenvolvimento Agroecológico do Cerrado,

Henriques RPB, 1993. Organização e estrutura das comunidades vegetais de cerrado em um gradiente topográfico no Brasil central. Tese de Doutorado. Campinas, SP: Instituto de Biologia. Universidade Estadual de Campinas.

Imanã-Encinas J, Paula JE De \& Sugimoto N, 2006. Análise do estrado arbóreo arbustivo jovem de uma área de Cerrado sensu stricto no município de Santa Quitéria, Maranhão. Boletim do Herbário Ezechias Paulo Heringer, 18:19-26.

Imaña-Encinas J, Paula JE De, Sugimoto N \& Imanãa-Encinas J, 1995. Análise fitossociológica do cerrado da fazenda Marflora. Pesquisa Agropecuária Brasileira, 30(5):577-582.

Ishara KL \& Maimoni-rodella RCS, 2012. Richness and similarity of the Cerrado vascular flora in the central west region of São Paulo state, Brazil. Check list, 8(1):32-42.

Ishara KL, 2010. Aspectos florísticos e estruturais de três fisionomias de Cerrado no município de Pratânia, São Paulo. Tese de Doutorado. Botucatu, SP: PPG Ciências Biológicas. Universidade Estadual PaulistaJúlio de Mesquita Filho.

Ishara KL, Déstro GFG, Maimoni-Rodella RCS \& Yanagizawa Y a. NP, 2008. Composição florística de remanescente de cerrado sensu stricto em Botucatu, SP. Revista Brasileira de Botânica, 31(4):575-586.

Jardim AVF, 2006. Previsão de guildas de dispersão e de fenologia foliar com base em atributos funcionais para espécies arbustivo-arbóreas em uma área de cerrado sensu stricto em Itirapina (SP). Dissertação de Mestrado. São Carlos, SP: PPG Ecologia e Recursos Naturais. Universidade Federal de São Carlos.

Latansio-Aidar SR, Oliveira ACP De, Rocha HR Da \& Aidar MPM, 2010. Fitossociologia de um cerrado denso em área de influência de torre de fluxo de carbono, Pé-de-Gigante, Parque Estadual de Vassununga, SP. Biota Neotropica, 10(1):195-207.

Lehn CR, Alves FM \& Damasceno-Júnior GA, 2008. Floríistica e fitissociologia de uma área de cerrado sensu stricto na região da borda oeste do Pantanal, Corumbá, MS, Brasil. Pesquisas botânica, 59:129-142.

Libano AM \& Felfili JM, 2006. Mudanças temporais na composição florística e na diversidade de um cerrado sensu stricto do Brasil Central em um período de 18 anos (1985-2003). Acta Botanica 
Brasilica, 20(4):927-936.

Lima E de S, Lima HS \& Ratter JA, 2009. Mudanças pós-fogo na estrutura e composição da vegetação lenhosa, em um cerrado mesotrófico, no período de cinco anos (1977-2002) em Nova Xavantina - MT. Cerne, 15(4):468-480.

Lima TA, Roberto J, Pinto R, et al., 2010. Florística e estrutura da vegetação arbustivo-arbórea em uma área de cerrado rupestre no Parque Estadual da Serra de Caldas Novas, Goiás. Biota Neotropica, 10(2):159-166.

Lima-Júnior GA, 2007. Flora e estrutura do estrato arbóreo e relações solo-vegetação de cerradão em Barão de Melgaço, Pantanal de Mato Grosso, Brasil. Dissertação de Mestrado. Viçosa, MG: PPG Botânica. Universidade Federal de Viçosa.

Lindoso G da S, 2008. Cerrado sensu stricto sobre neossolo quartzarênico: fitogeografia e conservação. Dissertação de Mestrado. Brasília, DF: PPG Ecologia. Universidade de Brasília.

Linsingen L Von, Sonehara J de S, Uhlmann A \& Cervi A, 2006. Composição florística do Parque Estadual do Cerrado de Jaguariaíva , Paraná , Brasil. Acta Biol. Par., 35(3-4):197-232.

Lisita VCV, 2008. Estudo de fragmento de cerrado stricto sensu em nove empreendimentos agropecuáarios de nordeste de Minas Gerais. Dissertação de Mestrado. Brasília, DF: PPG Ciências Florestais. Universidade de Brasília.

Lopes S de F, Vale VS Do \& Schiavini I, 2009. Efeito de queimadas sobre a estrutura e composição da comunidade vegetal lenhosa do cerrado sentido restrito em Caldas Novas, GO. Revista Árvore, 33(4):695-704.

Mantovani W, 1983. Composição e similaridade florística, fenologia e espectro biológico do cerrado da reserva biológica de Moji Guaçu, estado de São Paulo. Dissertação de Mestrado. Campinas, SP: Instituto de Biologia. Universidade Estadual de Campinas.

Marimon BS \& Lima EDS, 2001. Caracterizaçao fitofisionômica e levantamento florístico preliminar no Pantanal dos rios Morte-Araguaia, Cocalinho, Mato Grosso, Brasil. Acta Botanica Brasilica, 15(2):213-229.

Marimon BS, De S. Lima E, Duarte TG, Chieregatto LC \& Ratter JA, 2006. Observations on the vegetation of northeastern Mato Grosso, Brazil. IV. An analysis of the cerrado-amazonian forest ecotone. Edinburgh Journal of Botany, 63(2-3):323-341.

Marimon-Júnior BH \& Haridasan M, 2005. Comparação da vegetação arbórea e características edáficas de um cerradão e um cerrado sensu stricto em áreas adjacentes sobre solo distrófico no leste de Mato Grosso, Brasil. Acta Botanica Brasilica, 19(4):913-926.

Martins FQ, 2005. Sistemas de polinização em fragmentos de cerrado na região do Alto Taquari (GO, MS, MT). Dissertação de Mestrado. São Carlos, SP: PPG Ecologia e Recursos Naturais. Universidade Federal de São Carlos.

Matos $M$ de $Q$ \& Felfili JM, 2006. Composição florística e estrutura arbórea de fragmentos de cerrado sensu stricto na Bacia Hidrográfica do Rio São Bartolomeu, DF. In , XII Congresso de Iniciação Científica da UnB. XII Congresso de Iniciação Científica da UnB

Medeiros MB De, Guarino EDSG \& Silva GP, 2005. Fitossociologia de um trecho de cerrado sensu stricto na bacia do rio Corumbáa - área de influência direta do aproveitamento hidrelétrico Corumba IV (GO). Boletim de pesquisa e desenvolvimentoEmbrapa Recursos Genéticos e Biotecnologia,

Medeiros MB De, Walter BMT \& Silva GP, 2008. Fitossociologia do cerrado stricto sensu no município de Carolina, MA, Brasil. Cerne, 14(4):285-294.

Medeiros MM, Felfili JM \& Libano AM, 2007. Comparação florístico-estrutural dos estratos de regeneração e adulto em cerrado sensu stricto no Brasil central. Cerne, 13(3):291-298.

Meira-Neto JAA \& Saporetti-Júnior AW, 2002. Parâmetros fitossociológicos de um cerrado no parque nacional da Serra do Cipó, MG. Revista Árvore, 26(5):645-648. 
Meira-Neto JAA, 1991. Composição florística e fitossociologia de fisionomias de vegetação de cerrado sensu lato da Estação Ecológica de Santa Bárbara (EESB), município de Águas de Santa Bárbara, estado de São Paulo. Dissertação de Mestrado. Campinas, SP: Instituto de Biologia. Universidade Estadual de Campinas.

Meira-Neto JAA, Martins FR \& Valente GE, 2007. Composição florística e espectro biológico na estação ecológica de Santa Bárbara, estado de São Paulo, Brasil. Revista Árvore, 31(5):907-922.

Mendonça AH De, 2010. Avaliação do efeito de borda sobre a vegetação de cerrado stricto sensu inserido em matriz de pastagem. Dissertação de Mestrado. São Carlos, SP: Ciências da Engenharia Ambiental. Universidade de São Paulo.

Mendonça RC, Ribeiro JF \& Felfili JM, 2000. Levantamento Florístico da Fazenda São Francisco da Trijunção. Brasília-DF,

Mesquita MR \& Castro AAJF, 2007. Florística e fitossociologia de uma área de cerrado marginal (cerrado baixo), Parque Nacional de Sete Cidades, Piauí. Publicações avulsas em conservação de ecossistemas, 15:1-22.

Miranda IS, Almeida SS \& Dantas PJ, 2006. Florística e estrutura de comunidades arbóreas em cerrados de Rondônia,Brasil. Acta Amazonica, 36(4):419 - 430.

Miranda SC De, Silva-Júnior MC \& Salles $L$ de A, 2007. A comunidade lenhosa de cerrado rupestre na Serra Dourada, Goiás. Heringeriana, 1(1):43-54.

Moreno MIC \& Cardoso E, 2005. Utilização do método twinspan na delimitação de formações vegetacionais do cerrado 1. Caminhos de Geografia, 16(16):108-116.

Moreno MIC, Schiavini I \& Haridasan M, 2008. Fatores edáficos influenciando na estrutura de fitofisionomias do cerrado. Caminhos de Geografia, 9(25):173 - 194.

Moro M, Castro A \& Araújo F, 2011. Composição florística e estrutura de um fragmento de vegetação savânica sobre os tabuleiros pré-litorâneos na zona urbana de Fortaleza, Ceará. Rodriguésia, 62(2):407-423.

Moura I i O De, Gomes-Klein VL, Felfili JM \& Ferreira HD, 2007. Fitossociologia da comunidade lenhosa de uma área de cerrado rupestre no parque estadual dos Pirineus, Pirenópolis, Goiás. Revista de Biologia Neotropical, 4(2):83-100.

Neri AV, Meira-Neto JAA, Silva AF Da, Martins SV \& Saporetti-Júnior AW, 2007. Composição florística de uma área de cerrado sensu stricto no município de Senador Modestino Gonçalves, Vale do Jequitinhonha (MG) e análise de similaridade florística de algumas áreas de cerrado em Minas Gerais. Revista Árvore, 31(6):1109-1119.

Neri AV, Schaefer CEGR, Silva a. F, et al., 2012. the Influence of Soils on the Floristic Composition and Community Structure of an Area of Brazilian Cerrado Vegetation. Edinburgh Journal of Botany, 69(01):1-27.

Nogueira PE, Felfili JM, Da Silva Júnior MC, Delitti WBC \& Sevilha AC, 2001. Composição florística e fitossociologia de um cerrado sentido restrito no município de Canarana, MT. Boletim do Herbário Ezechias Paulo Heringer, 8:28-43.

Oliveira ACP De, Penha A dos S, Souza RF De \& Loiola MIB, 2012. Composição florística de uma comunidade savânica no Rio Grande do Norte, Nordeste do Brasil Material e métodos. Acta Botanica Brasilica, 26(3):559-569.

Oliveira FF \& Batalha MA, 2005. Lognormal abundance distribution of woody species in a cerrado fragment (São Carlos, southeastern Brazil). Revista Brasileira de Botânica, 28(1):39-45.

Oliveira MC De, Scolforo JRS, Mello JM De, Oliveira AD De \& Acerbi-Júnior FW, 2006. Avaliação de diferentes níveis de intervenção na florística, diversidade e similaridade de uma área de cerrado stricto sensu. Cerne, 12(4):342-349.

Oliveira ME de A, 2004. Mapeamento, florística e estrutura da transição campo-floresta na vegetação (cerrado) do parquem nacional de Sete Cidades, nordeste do Brasil. Tese de Doutorado. Campinas, SP: Institudo de Biologia. Universidade Estadual de Campinas. 
Oliveira-Filho AT \& Martins FR, 1991. A comparative study of five cerrado areas in southern Mato Grosso, Brazil. Edinburgh Journal of Botany, 48(3):307-332.

Oliveira-Filho AT, 1984. Estudo florístico e fitossociológico em um cerrado na Chapada dos Guimarães - Mato Grosso - uma análise de gradiente. Universidade Estadual de Campinas.

Oliveira-Filho AT, Shepherd GJGJ, Martins FR, Stubblebine WH \& Shephered GJ, 1989. Environmental factors affecting physiognomic and floristic variation in an area of cerrado in central Brazil. Journal of Tropical Ecology, 5(4):413-431.

Padilha DR, Salis SM \& Crispim SMA, 2008. Fitossociologia das Espécies Lenhosas em Campo Cerrado no Pantanal de Poconé e Paiaguás. EMBRAPA,

Paula JE De, Imanã-Encinas J \& Santana OA, 2007. Levantamento florístico e dendrométrico de um hectare de Cerrado sensu stricto em Planaltina, Distrito Federal. Revista Brasileira de Ciências Agrárias, 2(4):292-296.

Paula JE De, Imanã-Encinas J, Santana OA, Ribeiro GS \& Imaña CR, 2009. Levantamento florístico e sua distribuição diamétrica da vegetação de um cerrado sensu stricto e de um fragmento de floresta de galeria no ribeirão Dois Irmãos na APA Cafuringa, DF, Brasil. Biotemas, 22(3):35-46.

Pena MA, 2009. Florística de afloramentos rochosos na Serra do Cipó, Minas Gerais. Disertação de Mestrado. São Paulo, SP: PPG Botânica. Universidade de São Paulo.

Pereira-Silva EFL, 2003. Composição florística, estrutura fitossociológica e conteúdo de nitrogênio foliar em cerradão, Luís Antônio, SP. Dissertação de Mestrado. São Carlos, SP: PPG Ecologia e Recursos Naturais. Universidade Federal de São Carlos.

Pereira-Silva EFLL, Santos JE Dos, Kageyama PY \& Hardt E, 2004. Florística e fitossociologia dos estratos arbustivo e arbóreo de um remanescente de cerradão em uma Unidade de Conservação do Estado de São Paulo. Revista Brasileira de Botânica, 27(3):533-544.

Pessoa MJG, 2014. Composição Florística e Estrutura da Vegetação Lenhosa de Savanas Amazônicas Sobre Afloramentos Rochosos na Transição entre os Biomas. Dissertação de Mestrado. Nova Xavantina, MT: PPG Biodiversidade e Agroecossistemas Amazônicos. Universidade Estadual de Mato Grosso.

Pinheiro MHO, 2006. Composição e estrutura de uma comunidade savânica em gradiente topográfico no município de Corumbataí (SP, Brasil). Tese de Doutorado. Rio Claro, SP: Instituto de Biociências. Universidade Estadual Paulista Julio de Mesquita Filho, Camus Rio Claro.

Pinto JRR, 2008. Avaliação Ecológica Rápida da Reserva Natural Serra do Tombador: vegetação. Brasília, DF: Fundação O Boticário de Proteção à Natureza,

Pinto JRR, Lenza E, Pinto ADSES, Roberto J \& Pinto R, 2009. Composiçao florística e estrutura da vegetaçao arbustivo-arbórea em um cerrado rupestre, Cocalzinho de Goiás, GO. Revista Brasileira de Botânica, 32(1):1-29.

Pirani FR, Sanchez M \& Pedroni F, 2009. Fenologia de uma comunidade arbórea em cerrado restrito, Barra do Garças, MT, Brasil. Acta Botanica Brasilica, 23(4):1096-1109.

Ramos WM, 2009. Composição florística e síndrome de dispersão no morro Coração de Mãe, em Piraputanga, MS, Brasil. Dissertação de Mestrado. Campo Grande, MS: PPG Biologia Vegetal. Universidade Federal de Mato Grosso do Sul.

Ratter JA, Bridgewater S, Ribeiro JF, et al. Analysis of the floristic composition of the Brazilian cerrado vegetation IV: Presentation of a Revised Data-Base of 367 Areas. (http://cerrado.rbge.org.uk/cerrado/download/download.php).

Resende ILDM, Araújo GM De, Oliveira APDA \& Ávila Júnior RS De, 2004. A comunidade vegetal e as características abióticas de um campo de murundu em Uberlândia, MG. Acta Botanica Brasilica, 18(1):9-17.

Ribeiro JF, 1983. Comparação da concentração de nutrientes na vegetação arbórea e nos solos de um cerrado e um cerradão no Distrito Federal, Brasília, Brasil. Dissertação de Mestrado. PPG Ecologia. Universidade de Brasília. 
Ribeiro JF, Felfili JM, Walter BMT, et al., 2001. Caracterização florística e potencial de uso das espécies vasculares ocorrentes nas Fazendas Trijunção, BA. Planaltina-DF: EMBRAPA CERRADOS,

Ribeiro LF \& Tabarelli M, 2002. A structural gradient in cerrado vegetation of Brazil: changes in woody plant density, species richness, life history and plant composition. Journal of Tropical Ecology, 18:775-794.

Ritter LMO, 2008. Composição florística e aspectos físicos do cerrado nos campos gerais, Paraná. Dissertação de Mestrado. Ponta Grossa, PR: PPG Geociências. Universidade Estadual de Ponta Grossa.

Roitman I, Felfili JM \& Rezende A V., 2008. Tree dynamics of a fire-protected cerrado sensu stricto surrounded by forest plantations, over a 13-year period (1991-2004) in Bahia, Brazil. Plant Ecology, 197:255-267.

Rondon-Neto RM, Santos JS Dos, Silva MA Da \& Koppe VC, 2010. Potencialidades de uso de espécies arbustivas e arbóreas em diferentes fisionomias de cerrado, em Lucas do Rio Verde / MT. Revista de Biologia e Ciências da Terra, 10(2):113-126.

Roveratti J, 2008. Flora vascular do cerrado sensu stricto do parque nacional de Brasília, Distrito Federal, Brasil e chave para identificação das espécies. Dissertação de Mestrado. Brasília, DF: PPG Botânica. Universidade de Brasília.

Sá KLVR De, 2004. A flora vascular da reserva indígena São Jerônimo, São Jerônimo da Serra Paraná: subsídio para conservação da vegetação. Dissertação de Mestrado. Campinas, SP: Instituto de Biologia. Universidade Estadual de Campinas.

Sales H dos R, Santos RM Dos, Nunes YRF, Morais-Costa F \& Souza S da CA De, 2009. Caracterização florística de um fragmento de cerrado na APA estadual do rio Pandeiros - Bonito de Minas/MG. MG.BIOTA, 2(3):22-30.

Salis SM, Assis MA, Crispim SMA \& Casagrande JC, 2006. Distribuição e abundância de espécies arbóreas em cerradões no Pantanal, Estado do Mato Grosso do Sul, Brasil. Revista Brasileira de Botânica, 29(3):339-352.

Santana OA \& Imanãa-Encinas J, 2010. Fitossociologia das espécies arbóreas nativas de cerrado em áreas adjacentes a depósitos de resíduos domiciliares. Floresta, 40(1):93-110.

Santos ER Dos, 2000. Análise florística e estrutura fitossociológica da vegetação lenhosa de um trecho de cerrado stricto sensu do parque estadual do Lajeado, Palmas-TO. Dissertação de Mestrado. Viçosa, MG: PPG Botânica. Universidade Federal de Viçosa.

Santos JR \& Cavalcanti TB, 2005. Levantamento da flora vascular do parque ecológico e de uso múltplo Burle Marx (parque ecológico norte), Brasília, DF, Brasil. Boletim do Herbário Ezechias Paulo Heringer, 16:51-74.

Santos LGP Dos, 2008. Florística e conhecimento botânico tradicional em áreas de cerrado no município de Monsenhor Gil, Piauí, Brasil. Dissertação de Mestrado. Teresina, PI: PPG Desenvolvimento e Meio Ambiente. Universidade Federal do Piauí.

Santos RM Dos \& Vieira F de A, 2005. Análise estrutural do componente arbóreo de três área de cerrado em diferentes estádios de conservação no município de Três Marias, Minas Gerais, Brasil. Cerne, 11(4):399-408.

Santos TRR Dos, 2011. Fitogeografia da vegetação arbustivo-arbórea em áreas de cerrado rupestre no estado de Goiás. Dissertação de mestrado. Brasília, DF: PPG Ciências Florestais. Universidade de Brasília.

Saporetti-Júnior AW, Meira Neto JAA, Almado RDP \& Neto JAAM, 2003. Fitossociologia de cerrado sensu stricto no município de Abaeté-MG. Revista Árvore, 27(3):413-419.

Sarmento TR \& Silva-Júnior MC, 2006. Composição florística e fitossociologia das comunidades lenhosas do cerrado sentido restrito em duas posiçoes topográficas no Jardim Botânico de Brasília, DF. Boletim do Herbário Ezechias Paulo Heringer, 17:79-92. 
Sasaki D \& Mello-Silva R De, 2008. Levantamento florístico no cerrado de Pedregulho, SP, Brasil. Acta Botanica Brasilica, 22(1):187-202.

Sena ALM \& Pinto JRR, 2007. Levantamento fitossociológico da vegetação arbustivo-arbórea em um cerrado rupestre no município de Cristalina-GO. In Universidade de Brasília (ed.), XIII Congresso de Iniciação Científica da Universidade de Brasília. XIII Congresso de Iniciação Científica da Universidade de BrasíliaBrasília-DF,

Silva DM \& Batalha MA, 2008. Soil-vegetation relationships in cerrados under different fire frequencies. Plant and Soil, 311(1-2):87-96.

Silva FRG, 2006. Florística, estrutura e padrões de dispersão em um cerrado, Quartel General, MG. Dissertação de mestrado. Viçosa, MG: PPG Botânica. Universidade Federal de Viçosa.

Silva IA \& Batalha MA, 2009. Woody plant species co-occurrence in Brazilian savannas under different fire frequencies. Acta Oecologica, 36(1):85-91.

Silva IA, Cianciaruso MVV \& Batalha MA, 2009. Dispersal modes and fruiting periods in hyperseasonal and seasonal savannas, central Brazil. Revista Brasileira de Botânica, 32(1):155163.

Silva JS \& Felfili JM, 2012. Short communication Floristic composition of a conservation area in the Federal District of Brazil. Brazilian Journal of Botany, 35(4):385-395.

Silva JS, 2009. Diversidade alfa, florística e fitossociologia na ARIE do Cerradão na APA Gama e Cabeça de Veado, DF. Dissertação de Mestrado. Brasília, DF: PPG Botânica. Universidade de Brasília.

Silva LO, Costa DA, Santo-Filho KDE, et al., 2002. Levantamento florístico e fitossociológico em duas áreas de cerrado sensu stricto no Parque Estadual da Serra de Caldas Novas, Goiás. Acta Botanica Brasilica, 16(1):43-53.

Silveira EP Da, 2010. Florística e estrutura da vegetação de cerrado sensu stricto em terra indígena no nordeste do estado de Mato Grosso. Dissertação de Mestrado. Cuiabá-MT: PPG Ciências Florestais. Universidade Federal do Mato Grosso.

Sinimbu G, Porto AC, Damasceno CP, Souza FDR De \& Silva SR, 2007. Fitossociologia em Cerrado Sentido Restrito na Floresta Nacional de Brasília - DF. Revista Brasileira de Biociências, 5(2):1183-1185.

Siqueira AS, Araújo GM De \& Schiavini I, 2006. Caracterização florística da Reserva Particular do Patrimônio Natural (RPPN) Fazenda Carneiro, Lagamar, MG, Brasil. Biota Neotropica, 6(3).

Soares FM, Soares-Júnior FJ \& Teixeira A de P, 2002. Diversidade e similaridade florística em cinco fragmentos de cerrado no município de Itirapina, SP. In , XIV Congresso da Sociedade Botânica de São Paulo. XIV Congresso da Sociedade Botânica de São PauloRio Claro,

Souza AA, 2009a. Estudo de fitofisionomias de cerrado com dados de sensor hyperion/EO-1. Dissertação de Mestrado. São José dos Campos, SP: PPG Sensoriamento Remoto. Instituto Nacional de Pesquisas Espaciais.

Souza AJB, 2010. Estrutura e Dinâmica da Vegetação Lenhosa de Cerrado sensu stricto no período de 19 anos, na Reserva Ecológica do IBGE, Distrito Federal, Brasil. Dissertação de mestrado. Brasília, DF: PPG Ecologia. Universidade de Brasília.

Souza JT, Silva MAM, Mendes PGA, et al., 2007. Caracterização de uma vegetação de cerrado em uma área no município de Nova Olinda-CE. Cadernos de Cultura e Ciência, 2(2):2-12.

Souza LF De, 2009b. Diversidade florística e fenologia reprodutiva em fitofisionomias da reserva Pousada das Araras (município de Serranópolis, estado de Goiás, planalto central do Brasil). Tese de Doutorado. Rio Claro, SP: PPG Ciências Biológicas. Universidade Estadual Paulista Julio Mesquita Filho.

Tannus JLS, Assis M a. \& Morellato LPC, 2006. Fenologia reprodutiva em campo sujo e campo úmido numa área de Cerrado no sudeste do Brasil, Itirapina - SP. Biota Neotropica, 6(3):1-27.

Tartaglia D, 2004. Florística e fitossociologia das espécies lenhosas no cerrado da fazenda Canchim (São Carlos, SP). Dissertação de Mestrado. São Carlos, SP: PPG Ecologia e Recursos Naturais. 
Universidade Federal de São Carlos.

Teixeira MIJG, Araújo ARB, Valeri SV, Rodrigues RR \& Araujo ARB, 2004. Florística e fitossociologi de área de cerrado s.s. no município de Patrocínio Paulista, nordeste do estado de São Paulo. Bragantia, 63(1):1-11.

Toledo-Filho DV De, 1984. Composição florística e estrutura fitossociológica da vegetação de cerrado no município de Luis Antônio (SP). Tese de Doutorado. Campinas: Instituto de Biologia. Universidade Estadual de Campinas.

Toppa RH, 2004. Estrutura e diversidade florística das diferentes fisionomias de Cerrado e suas correlações com o solo na Estação Ecológica de Jataí , Luiz Antônio , SP. Tese de Doutorado. São Carlos, SP: PPG Ecologia e Recursos Naturais. Universidade Federal de São Carlos.

Uhlmann A, 2003. Análise estrutural de duas áreas de vegetação savânica (cerrado) sob influência de gradientes ambientais complexos. Tese de Doutorado. Campinas: PPG Biologia Vegetal. Universidade Estadual de Campinas.

Vale VS Do, Crespilho RF \& Schiavini I, 2009. Análise da regeneração natural em uma comunidade vegetal de cerrado no parque Victório Siquierolli, Uberlândia-MG. BioScience Journal, 25(1):131-145.

Valente EL, 2009. Relaçao solo-vegetaçao no parque nacional da Serra do Cipó, espinhaço meridional, Minas Gerais. Tese de Doutorado. Viçosa, MG: Solos e Nutriçao de Plantas. Universidade Federal de Viçosa.

Weiser V de L \& Godoy SAP De, 2001. Florística de um hectare de cerrado stricto sensu na ARIE Cerrado Pé-De-Gigante, Santa Rita do Passa Quatro, SP. Acta Botanica Brasilica, 15(2):201-212. 
583 Famílias e 909 espécies lenhosas registradas para as 625 localidades amostradas no bioma 584 Cerrado em fitofisionomia cerrado sentido amplo. As frequências relativas são rereferentes ao 585 número de localidades compiladas no presente estudo.

$586 \quad{ }^{1}$ Espécies dominantes no presente estudo.

$587{ }^{2}$ Espécies dominantes de acordo com Bridgewater et al. (2004).

588 *Fonte: JBRJ, (2014), Mendonça et al. (2008); Oliveira-Filho (2013), CRIA (2014).

\begin{tabular}{|c|c|c|c|c|c|}
\hline Família & & Espécie & $\begin{array}{c}\text { Freq. } \\
\text { Abs }\end{array}$ & $\begin{array}{c}\text { Freq. } \\
\text { Rel }\end{array}$ & Habito \\
\hline Anacardiaceae & & Anacardium giganteum W.Hancock ex Engl. & 1 & $0.2 \%$ & ARV \\
\hline Anacardiaceae & & Anacardium humile A.St.-Hil. & 20 & $3.2 \%$ & $\mathrm{ARB} / \mathrm{ARV}$ \\
\hline Anacardiaceae & 1,2 & Anacardium occidentale L. & 203 & $32.5 \%$ & ARV \\
\hline Anacardiaceae & 1,2 & Astronium fraxinifolium Schott & 304 & $48.6 \%$ & ARV \\
\hline Anacardiaceae & & Lithrea molleoides (Vell.) Engl. & 48 & $7.7 \%$ & $\mathrm{ARB} / \mathrm{ARV}$ \\
\hline Anacardiaceae & 2 & Myracrodruon urundeuva Allemão & 121 & $19.4 \%$ & ARV \\
\hline Anacardiaceae & & Schinopsis brasiliensis Engl. & 2 & $0.3 \%$ & ARV \\
\hline Anacardiaceae & & Schinus lentiscifolius Marchand & 2 & $0.3 \%$ & $\mathrm{ARB} / \mathrm{ARV}$ \\
\hline Anacardiaceae & & Schinus longifolius (Lindl.) Speg. & 1 & $0.2 \%$ & $\mathrm{ARB} / \mathrm{ARV}$ \\
\hline Anacardiaceae & & Schinus polygamus (Cav.) Cabrera & 3 & $0.5 \%$ & $\mathrm{ARB} / \mathrm{ARV}$ \\
\hline Anacardiaceae & & Schinus terebinthifolius Raddi & 23 & $3.7 \%$ & $\mathrm{ARB} / \mathrm{ARV}$ \\
\hline Anacardiaceae & & Spondias mombin L. & 4 & $0.6 \%$ & ARV \\
\hline Anacardiaceae & 1,2 & Tapirira guianensis Aubl. & 197 & $31.5 \%$ & ARV \\
\hline Anacardiaceae & & Tapirira obtusa (Benth.) J.D.Mitch. & 3 & $0.5 \%$ & ARV \\
\hline Annonaceae & & Annona cacans Warm. & 7 & $1.1 \%$ & ARV \\
\hline Annonaceae & 1,2 & Annona coriacea Mart. & 322 & $51.5 \%$ & $\mathrm{ARB} / \mathrm{ARV}$ \\
\hline Annonaceae & 1,2 & Annona crassiflora Mart. & 283 & $45.3 \%$ & ARV \\
\hline Annonaceae & & Annona emarginata (Schltdl.) H.Rainer & 5 & $0.8 \%$ & $\mathrm{ARB} / \mathrm{ARV}$ \\
\hline Annonaceae & & Annona jahnii Saff. & 1 & $0.2 \%$ & $\mathrm{ARB} / \mathrm{ARV}$ \\
\hline Annonaceae & & Annona leptopetala (R.E.Fr.) H.Rainer & 2 & $0.3 \%$ & $\mathrm{ARB} / \mathrm{ARV}$ \\
\hline Annonaceae & & Annona montana Macfad. & 1 & $0.2 \%$ & ARV \\
\hline Annonaceae & & Annona paludosa Aubl. & 3 & $0.5 \%$ & $\mathrm{ARB} / \mathrm{ARV}$ \\
\hline Annonaceae & & Annona sylvatica A.St.-Hil. & 6 & $1.0 \%$ & ARV \\
\hline Annonaceae & & Bocageopsis mattogrossensis (R.E.Fr.) R.E.Fr. & 8 & $1.3 \%$ & $\mathrm{ARB} / \mathrm{ARV}$ \\
\hline Annonaceae & & Cardiopetalum calophyllum Schltdl. & 41 & $6.6 \%$ & ARV \\
\hline Annonaceae & & Duguetia lanceolata A.St.-Hil. & 8 & $1.3 \%$ & ARV \\
\hline Annonaceae & & Duguetia marcgraviana Mart. & 11 & $1.8 \%$ & ARV \\
\hline Annonaceae & & Ephedranthus parviflorus S.Moore & 1 & $0.2 \%$ & ARV \\
\hline Annonaceae & & Ephedranthus pisocarpus R.E.Fr. & 6 & $1.0 \%$ & ARV \\
\hline Annonaceae & & Guatteria australis A.St.-Hil. & 8 & $1.3 \%$ & $\mathrm{ARB} / \mathrm{ARV}$ \\
\hline Annonaceae & & Guatteria blepharophylla Mart. & 2 & $0.3 \%$ & ARV \\
\hline Annonaceae & & Guatteria schomburgkiana Mart. & 1 & $0.2 \%$ & ARV \\
\hline Annonaceae & & Guatteria sellowiana Schltdl. & 8 & $1.3 \%$ & ARV \\
\hline Annonaceae & & Guatteria villosissima A.St.-Hil. & 1 & $0.2 \%$ & ARV \\
\hline Annonaceae & & Oxandra sessiliflora R.E.Fr. & 15 & $2.4 \%$ & $\mathrm{ARB} / \mathrm{ARV}$ \\
\hline Annonaceae & & Unonopsis guatterioides (A.DC.) R.E.Fr. & 7 & $1.1 \%$ & ARV \\
\hline Annonaceae & & Xylopia amazonica R.E.Fr. & 2 & $0.3 \%$ & ARV \\
\hline Annonaceae & 1,2 & Xylopia aromatica (Lam.) Mart. & 351 & $56.2 \%$ & ARB/ARV \\
\hline Annonaceae & & Xylopia benthamii R.E.Fr. & 1 & $0.2 \%$ & ARV \\
\hline Annonaceae & & Xylopia brasiliensis Spreng. & 13 & $2.1 \%$ & ARV \\
\hline Annonaceae & & Xylopia emarginata Mart. & 3 & $0.5 \%$ & ARV \\
\hline
\end{tabular}


Annonaceae

Annonaceae

Apocynaceae

Apocynaceae

Apocynaceae

Apocynaceae

Apocynaceae

Apocynaceae

Apocynaceae

Apocynaceae

Apocynaceae

Apocynaceae

Apocynaceae

Apocynaceae

Apocynaceae

Apocynaceae

Apocynaceae

Apocynaceae

Apocynaceae

Apocynaceae

Apocynaceae

Apocynaceae

Apocynaceae

Apocynaceae

Apocynaceae

Aquifoliaceae

Aquifoliaceae

Aquifoliaceae

Aquifoliaceae

Aquifoliaceae

Aquifoliaceae

Araliaceae

Araliaceae

Araliaceae

Araliaceae

Araliaceae

Asteraceae

Asteraceae

Asteraceae

Asteraceae

Asteraceae

Asteraceae

Asteraceae

Asteraceae

Asteraceae

Asteraceae

Asteraceae

Asteraceae

Asteraceae

Asteraceae

Asteraceae

Asteraceae

Asteraceae

Asteraceae

Asteraceae

Asteraceae
Xylopia frutescens Aubl.

Xylopia sericea A.St.-Hil.

Aspidosperma australe Müll.Arg.

Aspidosperma cuspa (Kunth) S.F.Blake ex Pittier

Aspidosperma discolor A.DC.

1,2 Aspidosperma macrocarpon Mart.

Aspidosperma multiflorum A.DC.

Aspidosperma nobile Müll.Arg.

Aspidosperma parvifolium A.DC.

Aspidosperma polyneuron Müll.Arg.

Aspidosperma pyrifolium Mart.

Aspidosperma ramiflorum Müll.Arg.

Aspidosperma spruceanum Benth. ex Müll.Arg.

2 Aspidosperma subincanum Mart.

1,2 Aspidosperma tomentosum Mart.

Geissospermum laeve (Vell.) Miers

1,2 Hancornia speciosa Gomes

Himatanthus articulatus (Vahl) Woodson

Himatanthus bracteatus (A.DC.) Woodson

Himatanthus drasticus (Mart.) Plumel

1,2 Himatanthus obovatus (Müll.Arg.) Woodson

Himatanthus phagedaenicus (Mart.) Woodson

Tabernaemontana catharinensis A.DC.

Tabernaemontana hystrix Steud.

Tabernaemontana solanifolia A.DC.

Ilex affinis Gardner

Ilex cerasifolia Reissek

Ilex congesta Reissek

Ilex conocarpa Reissek

Ilex paraguariensis A.St.-Hil.

Ilex theezans Mart. ex Reissek

Dendropanax cuneatus (DC.) Decne. \& Planch.

Schefflera burchellii (Seem.) Frodin \& Fiaschi

Schefflera longipetiolata (Pohl ex DC.) Frodin \& Fiaschi

1,2 Schefflera macrocarpa (Cham. \& Schltdl.) Frodin

Schefflera morototoni (Aubl.) Maguire et al.

Dasyphyllum brasiliense (Spreng.) Cabrera

Eremanthus arboreus (Gardner) MacLeish

Eremanthus argenteus MacLeish \& H.Schumach.

Eremanthus brasiliensis (Gardner) MacLeish

Eremanthus capitatus (Spreng.) MacLeish

Eremanthus elaeagnus (Mart. ex DC.) Sch.Bip.

Eremanthus erythropappus (DC.) MacLeish

Eremanthus glomeratus Less.

Eremanthus goyazensis (Gardner) Sch.Bip.

Eremanthus incanus (Less.) Less.

Eremanthus mattogrossensis Kuntze

Eremanthus polycephalus (DC.) MacLeish

Eremanthus seidelii MacLeish \& Schumacher

Lychnophora ericoides Mart.

Lychnophora salicifolia Mart.

Lychnophora tomentosa (Mart. ex DC.) Sch.Bip.

Piptocarpha axillaris (Less.) Baker

Piptocarpha macropoda (DC.) Baker

1,2 Piptocarpha rotundifolia (Less.) Baker

Vernonanthura discolor (Spreng.) H.Rob.

$\begin{array}{rrl}1 & 0.2 \% & \text { ARB/ARV } \\ 51 & 8.2 \% & \text { ARB/ARV } \\ 10 & 1.6 \% & \text { ARV } \\ 4 & 0.6 \% & \text { ARB/ARV } \\ 9 & 1.4 \% & \text { ARB/ARV } \\ 237 & 37.9 \% & \text { ARV } \\ 35 & 5.6 \% & \text { ARB/ARV } \\ 100 & 16.0 \% & \text { ARV } \\ 6 & 1.0 \% & \text { ARV } \\ 4 & 0.6 \% & \text { ARV } \\ 7 & 1.1 \% & \text { ARV } \\ 3 & 0.5 \% & \text { ARV } \\ 8 & 1.3 \% & \text { ARV } \\ 108 & 17.3 \% & \text { ARV } \\ 330 & 52.8 \% & \text { ARV } \\ 1 & 0.2 \% & \text { ARV } \\ 320 & 51.2 \% & \text { ARV } \\ 49 & 7.8 \% & \text { ARV } \\ 2 & 0.3 \% & \text { ARV } \\ 8 & 1.3 \% & \text { ARV } \\ 306 & 49.0 \% & \text { ARV } \\ 2 & 0.3 \% & \text { ARV } \\ 5 & 0.8 \% & \text { ARB/ARV } \\ 6 & 1.0 \% & \text { ARV } \\ 2 & 0.3 \% & \text { ARV } \\ 6 & 1.0 \% & \text { ARB/ARV } \\ 11 & 1.8 \% & \text { ARV } \\ 2 & 0.3 \% & \text { ARV } \\ 8 & 1.3 \% & \text { ARB/ARV } \\ 1 & 0.2 \% & \text { ARB/ARV } \\ 2 & 0.3 \% & \text { ARB/ARV } \\ 3 & 0.5 \% & \text { ARV } \\ 1 & 0.2 \% & \text { ARB/ARV } \\ 1 & 0.2 \% & \text { ARV }\end{array}$

$148 \quad 23.7 \%$ ARB/ARV

$23 \quad 3.7 \%$ ARV

$10.2 \%$ ARB/ARV

$20.3 \%$ ARV

$20.3 \%$ ARV

$10.2 \%$ ARV

$6 \quad 1.0 \%$ ARV

$1 \quad 0.2 \%$ ARV

$14 \quad 2.2 \%$ ARV

$80 \quad 12.8 \%$ ARV

$20 \quad 3.2 \%$ ARV

$4 \quad 0.6 \%$ ARV

$16 \quad 2.6 \%$ ARB/ARV

$5 \quad 0.8 \% \quad$ ARV

$20.3 \%$ ARB/ARV

$6 \quad 1.0 \%$ ARV

$10.2 \%$ ARV

$1 \quad 0.2 \%$ ARV

$4 \quad 0.6 \%$ ARV

$5 \quad 0.8 \% \quad$ ARV

$230 \quad 36.8 \%$ ARV

$5 \quad 0.8 \%$ ARV 
Asteraceae

Asteraceae

Asteraceae

Bignoniaceae

Bignoniaceae

Bignoniaceae

Bignoniaceae

Bignoniaceae

Bignoniaceae

Bignoniaceae

Bignoniaceae

Bignoniaceae

Bignoniaceae

Bignoniaceae

Bignoniaceae

Bignoniaceae

Bignoniaceae

Bignoniaceae

Bignoniaceae

Bignoniaceae

Bignoniaceae

Bignoniaceae

Bixaceae

Bixaceae

Bixaceae

Boraginaceae

Boraginaceae

Boraginaceae

Boraginaceae

Boraginaceae

Boraginaceae

Boraginaceae

Boraginaceae

Boraginaceae

Boraginaceae

Boraginaceae

Burseraceae

Burseraceae

Burseraceae

Burseraceae

Burseraceae

Burseraceae

Burseraceae

Burseraceae

Burseraceae

Calophyllaceae

Calophyllaceae

Calophyllaceae

Calophyllaceae

Calophyllaceae

Calophyllaceae

Calophyllaceae

Calophyllaceae

Calophyllaceae

Calophyllaceae

Cannabaceae
Vernonanthura phosphorica (Vell.) H.Rob.

Wunderlichia cruelsiana Taub.

Wunderlichia mirabilis Riedel ex Baker

1,2 Cybistax antisyphilitica (Mart.) Mart.

Godmania aesculifolia (Kunth) Standl.

Handroanthus chrysotrichus (Mart. exDC.) Mattos

Handroanthus impetiginosus Mattos

1,2 Handroanthus ochraceus (Cham.) Mattos

Handroanthus pulcherrimus (Sandwith) Mattos

Handroanthus serratifolius (A.H.Gentry) S.Grose

Jacaranda brasiliana (Lam.) Pers.

Jacaranda cuspidifolia Mart.

Jacaranda jasminoides (Thunb.) Sandwith

Jacaranda micrantha Cham.

Jacaranda mimosifolia D. Don

Jacaranda praetermissa Sandwith

Jacaranda puberula Cham.

Jacaranda simplicifolia K.Schum. ex Bureau \& K.Schum.

1,2 Tabebuia aurea (Silva Manso) Benth. \& Hook.f. ex S.Moore

Tabebuia insignis (Miq.) Sandwith

Tabebuia roseoalba (Ridl.) Sandwith

1,2 Zeyheria montana Mart.

Bixa orellana L.

Cochlospermum orinocense (Kunth) Steud.

Cochlospermum vitifolium (Willd.) Spreng.

Cordia alliodora (Ruiz \& Pav.) Cham.

Cordia bicolor A.DC.

Cordia ecalyculata Vell.

Cordia glabrata (Mart.) A.DC.

Cordia nodosa Lam.

Cordia rufescens A.DC.

Cordia scabrifolia A.DC.

Cordia sellowiana Cham.

Cordia superba Cham.

Cordia trichotoma (Vell.) Arráb. ex Steud.

Jacaranda mutabilis Hassl.

Commiphora leptophloeos (Mart.) J.B.Gillett

Protium brasiliense (Spreng.) Engl.

1,2 Protium heptaphyllum (Aubl.) Marchand

2 Protium ovatum Engl.

Protium pallidum Cuatrec.

Protium pilosissimum Engl.

Protium spruceanum (Benth.) Engl.

Protium unifoliolatum Engl.

Tetragastris altissima (Aubl.) Swart

Calophyllum brasiliense Cambess.

Caraipa savannarum Kubitzki

1,2 Kielmeyera coriacea Mart. \& Zucc.

Kielmeyera grandiflora (Wawra) Saddi

Kielmeyera lathrophyton Saddi

Kielmeyera paranaensis Saddi

Kielmeyera petiolaris Mart.

Kielmeyera rosea Mart. \& Zucc.

1,2 Kielmeyera rubriflora Cambess.

2 Kielmeyera speciosa A.St.-Hil.

Celtis iguanaea (Jacq.) Sarg.

\begin{tabular}{|c|c|c|}
\hline 15 & $2.4 \%$ & ARB/ARV \\
\hline 11 & $1.8 \%$ & $\mathrm{ARB} / \mathrm{ARV}$ \\
\hline 15 & $2.4 \%$ & $\mathrm{ARV}$ \\
\hline 167 & $26.7 \%$ & ARV \\
\hline 1 & $0.2 \%$ & ARV \\
\hline 6 & $1.0 \%$ & ARV \\
\hline 49 & $7.8 \%$ & ARV \\
\hline 409 & $65.4 \%$ & ARV \\
\hline 1 & $0.2 \%$ & ARV \\
\hline 114 & $18.2 \%$ & ARV \\
\hline 72 & $11.5 \%$ & ARV \\
\hline 47 & $7.5 \%$ & ARV \\
\hline 2 & $0.3 \%$ & ARV \\
\hline 4 & $0.6 \%$ & ARV \\
\hline 1 & $0.2 \%$ & ARV \\
\hline 1 & $0.2 \%$ & ARV \\
\hline 3 & $0.5 \%$ & ARV \\
\hline 1 & $0.2 \%$ & ARV \\
\hline 380 & $60.8 \%$ & ARV \\
\hline 3 & $0.5 \%$ & ARV \\
\hline 51 & $8.2 \%$ & ARV \\
\hline 154 & $24.6 \%$ & $\mathrm{ARB} / \mathrm{ARV}$ \\
\hline 1 & $0.2 \%$ & $\mathrm{ARB} / \mathrm{ARV}$ \\
\hline 3 & $0.5 \%$ & ARV \\
\hline 32 & $5.1 \%$ & $\mathrm{ARB} / \mathrm{ARV}$ \\
\hline 4 & $0.6 \%$ & ARV \\
\hline 1 & $0.2 \%$ & ARV \\
\hline 2 & $0.3 \%$ & $\mathrm{ARV}$ \\
\hline 50 & $8.0 \%$ & $\mathrm{ARB} / \mathrm{ARV}$ \\
\hline 1 & $0.2 \%$ & $\mathrm{ARB} / \mathrm{ARV}$ \\
\hline 6 & $1.0 \%$ & $\mathrm{ARB} / \mathrm{ARV}$ \\
\hline 1 & $0.2 \%$ & ARV \\
\hline 19 & $3.0 \%$ & ARV \\
\hline 1 & $0.2 \%$ & $\mathrm{ARB} / \mathrm{ARV}$ \\
\hline 22 & $3.5 \%$ & ARV \\
\hline 1 & $0.2 \%$ & ARV \\
\hline 2 & $0.3 \%$ & $\mathrm{ARB} / \mathrm{ARV}$ \\
\hline 8 & $1.3 \%$ & $\mathrm{ARB} / \mathrm{ARV}$ \\
\hline 187 & $29.9 \%$ & $\mathrm{ARB} / \mathrm{ARV}$ \\
\hline 30 & $4.8 \%$ & $\mathrm{ARB} / \mathrm{ARV}$ \\
\hline 1 & $0.2 \%$ & ARV \\
\hline 3 & $0.5 \%$ & $\mathrm{ARB} / \mathrm{ARV}$ \\
\hline 12 & $1.9 \%$ & ARV \\
\hline 4 & $0.6 \%$ & ARB/ARV \\
\hline 21 & $3.4 \%$ & ARV \\
\hline 9 & $1.4 \%$ & ARV \\
\hline 1 & $0.2 \%$ & ARV \\
\hline 388 & $62.1 \%$ & SUB/ARB/ARV \\
\hline 13 & $2.1 \%$ & $\mathrm{ARB} / \mathrm{ARV}$ \\
\hline 86 & $13.8 \%$ & $\mathrm{ARB} / \mathrm{ARV}$ \\
\hline 2 & $0.3 \%$ & $\mathrm{ARB} / \mathrm{ARV}$ \\
\hline 5 & $0.8 \%$ & ARV \\
\hline 6 & $1.0 \%$ & SUB/ARV \\
\hline 130 & $20.8 \%$ & SUB/ARB/ARV \\
\hline 87 & $13.9 \%$ & $\mathrm{ARB} / \mathrm{ARV}$ \\
\hline 1 & $0.2 \%$ & $\mathrm{ARB} / \mathrm{ARV}$ \\
\hline
\end{tabular}


Cannabaceae

Caryocaraceae

Caryocaraceae

Caryocaraceae

Caryocaraceae

Celastraceae

Celastraceae

Celastraceae

Celastraceae

Celastraceae

Celastraceae

Celastraceae

Celastraceae

Celastraceae

Celastraceae

Chrysobalanaceae

Chrysobalanaceae

Chrysobalanaceae

Chrysobalanaceae

Chrysobalanaceae

Chrysobalanaceae

Chrysobalanaceae

Chrysobalanaceae

Chrysobalanaceae

Chrysobalanaceae

Chrysobalanaceae

Chrysobalanaceae

Chrysobalanaceae

Chrysobalanaceae

Chrysobalanaceae

Chrysobalanaceae

Chrysobalanaceae

Chrysobalanaceae

Chrysobalanaceae

Clethraceae

Clusiaceae

Clusiaceae

Clusiaceae

Clusiaceae

Clusiaceae

Clusiaceae

Combretaceae

Combretaceae

Combretaceae

Combretaceae

Combretaceae

Combretaceae

Combretaceae

Combretaceae

Combretaceae

Combretaceae

Combretaceae

Combretaceae

Combretaceae

Connaraceae

Connaraceae
Trema micrantha (L.) Blume

1,2 Caryocar brasiliense Cambess.

Caryocar coriaceum Wittm.

2 Caryocar cuneatum Wittm.

Caryocar glabrum (Aubl.) Pers.

Cheiloclinium cognatum (Miers) A.C.Sm.

Maytenus communis Reissek

Maytenus evonymoides Reissek

Maytenus floribunda Reissek

Maytenus gonoclada Mart.

Peritassa laevigata (Hoffmanns. ex Link) A.C.Sm.

1,2 Plenckia populnea Reissek

1,2 Salacia crassifolia (Mart. ex Schult.) G.Don

Salacia elliptica (Mart. ex Schult.) G.Don

Tontelea micrantha (Mart. ex Schult.) A.C.Sm.

1,2 Couepia grandiflora (Mart. \& Zucc.) Benth.

Exellodendron cordatum (Hook.f.) Prance

2 Hirtella ciliata Mart. \& Zucc.

Hirtella glandulosa Spreng.

Hirtella gracilipes (Hook.f.) Prance

Hirtella hebeclada Moric. ex DC.

Hirtella racemosa Lam.

Licania apetala (E.Mey.) Fritsch

Licania dealbata Hook.f.

Licania gardneri (Hook.f.) Fritsch

Licania hoehnei Pilg.

2 Licania humilis Cham. \& Schltdl.

Licania kunthiana Hook.f.

Licania minutiflora (Sagot) Fritsch

Licania octandra (Hoffmanns. ex Roem. \& Schult.) Kuntze

Licania parviflora Benth.

Licania rigida Benth.

Licania sclerophylla (Hook.f.) Fritsch

Parinari excelsa Sabine

Clethra scabra Pers.

Clusia burchellii Engl.

Clusia criuva Cambess.

Clusia weddelliana Planch. \& Triana

Garcinia brasiliensis Mart.

Garcinia gardneriana (Planch. \& Triana) Zappi

Platonia insignis Mart.

Buchenavia grandis Ducke

Buchenavia tetraphylla (Aubl.) R.A.Howard

1 Buchenavia tomentosa Eichler

Combretum glaucocarpum Mart.

Combretum laxum Jacq.

Combretum leprosum Mart.

Combretum mellifluum Eichler

Terminalia actinophylla Mart.

1,2 Terminalia argentea Mart.

2 Terminalia fagifolia Mart.

Terminalia glabrescens Mart.

Terminalia januariensis DC.

Terminalia phaeocarpa Eichler

Connarus perrottetii (DC.) Planch.

1,2 Connarus suberosus Planch.
$132.1 \%$ ARB/ARV

$352 \quad 56.3 \%$ ARV

$6911.0 \%$ ARV

$56 \quad 9.0 \%$ ARV

$10.2 \% \quad$ ARV

$12 \quad 1.9 \%$ LIA/ARB/ARV

$1 \quad 0.2 \% \quad \mathrm{ARB} / \mathrm{ARV}$

$30.5 \% \quad \mathrm{ARB} / \mathrm{ARV}$

$20.3 \%$ ARV

$7 \quad 1.1 \% \quad$ ARB/ARV

$1 \quad 0.2 \%$ LIA/ARV

$20032.0 \%$ ARV

$198 \quad 31.7 \% \quad$ SUB/ARB/ARV

$59 \quad 9.4 \% \quad$ SUB/ARB/ARV

$11 \quad 1.8 \%$ SUB/ARB/ARV

$278 \quad 44.5 \%$ ARV

$14 \quad 2.2 \% \quad$ ARB/ARV

$94 \quad 15.0 \%$ ARV

$109 \quad 17.4 \%$ ARV

$36 \quad 5.8 \% \quad \mathrm{ARB} / \mathrm{ARV}$

$10.2 \% \quad$ ARV

$4 \quad 0.6 \% \quad \mathrm{ARB} / \mathrm{ARV}$

$12 \quad 1.9 \% \quad \mathrm{ARB} / \mathrm{ARV}$

$1 \quad 0.2 \% \quad \mathrm{ARB} / \mathrm{ARV}$

$23 \quad 3.7 \% \quad$ ARB/ARV

$1 \quad 0.2 \% \quad \mathrm{ARV}$

$90 \quad 14.4 \% \quad$ ARB/ARV

$8 \quad 1.3 \% \quad$ ARV

$4 \quad 0.6 \%$ ARV

$5 \quad 0.8 \%$ ARV

$6 \quad 1.0 \%$ ARV

$7 \quad 1.1 \%$ ARV

$16 \quad 2.6 \%$ ARV

$1 \quad 0.2 \%$ ARV

$121.9 \% \quad \mathrm{ARB} / \mathrm{ARV}$

$10.2 \% \quad$ ARV

$10.2 \% \quad$ ARB/ARV

$30.5 \%$ ARV

$20.3 \% \quad \mathrm{ARB} / \mathrm{ARV}$

$1 \quad 0.2 \% \quad \mathrm{ARB} / \mathrm{ARV}$

$20 \quad 3.2 \%$ ARV

$10.2 \%$ ARV

$12 \quad 1.9 \%$ ARV

$147 \quad 23.5 \%$ ARV

$20.3 \% \quad$ ARB/ARV

$2 \quad 0.3 \% \quad$ LIA/ARB/ARV

$193.0 \% \quad$ LIA/ARB/ARV

$35 \quad 5.6 \%$ LIA/ARB/ARV

$4 \quad 0.6 \%$ ARV

$246 \quad 39.4 \%$ ARB/ARV

123 19.7\% ARV

63 10.1\% ARB/ARV

$10.2 \%$ ARV

$9 \quad 1.4 \%$ ARV

$6 \quad 1.0 \% \quad$ ARV

$422 \quad 67.5 \% \quad \mathrm{ARB} / \mathrm{ARV}$ 
Cunoniaceae

Dichapetalaceae

Dilleniaceae

Dilleniaceae

Ebenaceae

Ebenaceae

Ebenaceae

Ebenaceae

Ebenaceae

Ebenaceae

Ebenaceae

Elaeocarpaceae

Elaeocarpaceae

Elaeocarpaceae

Ericaceae

Erythroxylaceae

Erythroxylaceae

Erythroxylaceae

Erythroxylaceae

Erythroxylaceae

Erythroxylaceae

Erythroxylaceae

Erythroxylaceae

Erythroxylaceae

Erythroxylaceae

Erythroxylaceae

Erythroxylaceae

Erythroxylaceae

Erythroxylaceae

Erythroxylaceae

Erythroxylaceae

Erythroxylaceae

Euphorbiaceae

Euphorbiaceae

Euphorbiaceae

Euphorbiaceae

Euphorbiaceae

Euphorbiaceae

Euphorbiaceae

Euphorbiaceae

Euphorbiaceae

Euphorbiaceae

Euphorbiaceae

Euphorbiaceae

Euphorbiaceae

Euphorbiaceae

Euphorbiaceae

Euphorbiaceae

Euphorbiaceae

Euphorbiaceae

Euphorbiaceae

Euphorbiaceae

Euphorbiaceae

Euphorbiaceae

Euphorbiaceae

Euphorbiaceae
Lamanonia ternata Vell.

Tapura amazonica Poepp. \& Endl.

1,2 Curatella americana L.

1,2 Davilla elliptica A.St.-Hil.

Diospyros brasiliensis Mart. ex Miq.

Diospyros coccolobifolia Mart. ex Miq.

1,2 Diospyros hispida A.DC.

Diospyros inconstans Jacq.

Diospyros obovata Jacq.

Diospyros sericea A.DC.

Diospyros tetrasperma Sw.

Sloanea eichleri K.Schum.

Sloanea guianensis (Aubl.) Benth.

Sloanea sinemariensis Aubl.

Gaylussacia brasiliensis (Spreng.) Meisn.

Erythroxylum ambiguum Peyr.

Erythroxylum amplifolium (Mart.) O.E.Schulz

Erythroxylum anguifugum Mart.

Erythroxylum barbatum O.E.Schulz

Erythroxylum buxus Peyr.

Erythroxylum campestre A.St.-Hil.

Erythroxylum citrifolium A.St.-Hil.

Erythroxylum daphnites Mart.

1 Erythroxylum deciduum A.St.-Hil.

Erythroxylum engleri O.E.Schulz

Erythroxylum foetidum Plowman

Erythroxylum pelleterianum A.St.-Hil.

Erythroxylum rufum Cav.

Erythroxylum squamatum Sw.

1,2 Erythroxylum suberosum A.St.-Hil.

Erythroxylum subracemosum Turcz.

1,2 Erythroxylum tortuosum Mart.

Actinostemon klotzschii (Didr.) Pax

Alchornea discolor Poepp.

Alchornea triplinervia (Spreng.) Müll.Arg.

Cnidoscolus vitifolius (Mill.) Pohl

Croton blanchetianus Baill.

Croton floribundus Spreng.

Croton macrobothrys Baill.

Croton sonderianus Müll.Arg.

Croton urucurana Baill.

Gymnanthes klotzschiana Müll.Arg.

Hevea brasiliensis (Willd. ex A.Juss.) Müll.Arg.

Mabea fistulifera Mart.

Mabea pohliana (Benth.) Müll.Arg.

Manihot anomala Pohl

Manihot caerulescens Pohl

Manihot carthaginensis (Jacq.) Müll.Arg.

Manihot tripartita (Spreng.) Müll.Arg.

Maprounea brasiliensis A.St.-Hil.

1,2 Maprounea guianensis Aubl.

Pleradenophora membranifolia (Müll. Arg.) Esser \&. A. L. Melo

Sapium argutum (Müll.Arg.) Huber

Sapium glandulosum (L.) Morong

Sapium haematospermum Müll.Arg.

Sebastiania brasiliensis Spreng.

\begin{tabular}{|c|c|c|}
\hline 10 & $1.6 \%$ & $\mathrm{ARB} / \mathrm{ARV}$ \\
\hline 36 & $5.8 \%$ & ARV \\
\hline 353 & $56.5 \%$ & $\mathrm{ARB} / \mathrm{ARV}$ \\
\hline 342 & $54.7 \%$ & LIA/ARB/ARV \\
\hline 4 & $0.6 \%$ & ARV \\
\hline 49 & $7.8 \%$ & ARV \\
\hline 361 & $57.8 \%$ & $\mathrm{ARB} / \mathrm{ARV}$ \\
\hline 9 & $1.4 \%$ & ARV \\
\hline 1 & $0.2 \%$ & ARV \\
\hline 76 & $12.2 \%$ & $\mathrm{ARB} / \mathrm{ARV}$ \\
\hline 1 & $0.2 \%$ & ARV \\
\hline 1 & $0.2 \%$ & ARV \\
\hline 3 & $0.5 \%$ & ARV \\
\hline 1 & $0.2 \%$ & ARV \\
\hline 1 & $0.2 \%$ & SUB/ARB/ARV \\
\hline 8 & $1.3 \%$ & ARB/ARV \\
\hline 7 & $1.1 \%$ & $\mathrm{ARB} / \mathrm{ARV}$ \\
\hline 9 & $1.4 \%$ & $\mathrm{ARB} / \mathrm{ARV}$ \\
\hline 4 & $0.6 \%$ & $\mathrm{ARB} / \mathrm{ARV}$ \\
\hline 4 & $0.6 \%$ & $\mathrm{ARB} / \mathrm{ARV}$ \\
\hline 34 & $5.4 \%$ & SUB/ARB/ARV \\
\hline 5 & $0.8 \%$ & $\mathrm{ARB} / \mathrm{ARV}$ \\
\hline 65 & $10.4 \%$ & $\mathrm{ARB} / \mathrm{ARV}$ \\
\hline 176 & $28.2 \%$ & SUB/ARB/ARV \\
\hline 17 & $2.7 \%$ & $\mathrm{ARB} / \mathrm{ARV}$ \\
\hline 2 & $0.3 \%$ & $\mathrm{ARV}$ \\
\hline 12 & $1.9 \%$ & $\mathrm{ARB} / \mathrm{ARV}$ \\
\hline 1 & $0.2 \%$ & $\mathrm{ARB} / \mathrm{ARV}$ \\
\hline 2 & $0.3 \%$ & ARB/ARV \\
\hline 395 & $63.2 \%$ & SUB/ARB/ARV \\
\hline 3 & $0.5 \%$ & $\mathrm{ARB} / \mathrm{ARV}$ \\
\hline 198 & $31.7 \%$ & $\mathrm{ARB} / \mathrm{ARV}$ \\
\hline 2 & $0.3 \%$ & $\mathrm{ARB} / \mathrm{ARV}$ \\
\hline 32 & $5.1 \%$ & $\mathrm{ARB} / \mathrm{ARV}$ \\
\hline 10 & $1.6 \%$ & $\mathrm{ARB} / \mathrm{ARV}$ \\
\hline 20 & $3.2 \%$ & $\mathrm{ARB} / \mathrm{ARV}$ \\
\hline 1 & $0.2 \%$ & $\mathrm{ARB} / \mathrm{ARV}$ \\
\hline 10 & $1.6 \%$ & ARV \\
\hline 1 & $0.2 \%$ & ARV \\
\hline 1 & $0.2 \%$ & $\mathrm{ARB} / \mathrm{ARV}$ \\
\hline 6 & $1.0 \%$ & ARV \\
\hline 1 & $0.2 \%$ & ARB/ARV \\
\hline 1 & $0.2 \%$ & ARV \\
\hline 32 & $5.1 \%$ & $\mathrm{ARB} / \mathrm{ARV}$ \\
\hline 10 & $1.6 \%$ & $\mathrm{ARB} / \mathrm{ARV}$ \\
\hline 1 & $0.2 \%$ & SUB/LIA/ARB/ARV \\
\hline 12 & $1.9 \%$ & SUB/LIA/ARB/ARV \\
\hline 2 & $0.3 \%$ & $\mathrm{ARB} / \mathrm{ARV}$ \\
\hline 11 & $1.8 \%$ & SUB/ARB/ARV \\
\hline 2 & $0.3 \%$ & ARB/ARV \\
\hline 132 & $21.1 \%$ & $\mathrm{ARB} / \mathrm{ARV}$ \\
\hline 1 & $0.2 \%$ & $\mathrm{ARB} / \mathrm{ARV}$ \\
\hline 1 & $0.2 \%$ & $\mathrm{ARB} / \mathrm{ARV}$ \\
\hline 25 & $4.0 \%$ & $\mathrm{ARB} / \mathrm{ARV}$ \\
\hline 19 & $3.0 \%$ & $\mathrm{ARB} / \mathrm{ARV}$ \\
\hline 11 & $1.8 \%$ & $\mathrm{ARB} / \mathrm{ARV}$ \\
\hline
\end{tabular}


Euphorbiaceae

Fabaceae

Fabaceae

Fabaceae

Fabaceae

Fabaceae

Fabaceae

Fabaceae

Fabaceae

Fabaceae

Fabaceae

Fabaceae

Fabaceae

Fabaceae

Fabaceae

Fabaceae

Fabaceae

Fabaceae

Fabaceae

Fabaceae

Fabaceae

Fabaceae

Fabaceae

Fabaceae

Fabaceae

Fabaceae

Fabaceae

Fabaceae

Fabaceae

Fabaceae

Fabaceae

Fabaceae

Fabaceae

Fabaceae

Fabaceae

Fabaceae

Fabaceae

Fabaceae

Fabaceae

Fabaceae

Fabaceae

Fabaceae

Fabaceae

Fabaceae

Fabaceae

Fabaceae

Fabaceae

Fabaceae

Fabaceae

Fabaceae

Fabaceae

Fabaceae

Fabaceae

Fabaceae

Fabaceae

Fabaceae
Sebastiania serrata (Baill. ex Müll.Arg.) Müll.Arg. Abarema langsdorffii (Benth.) Barneby \& J.W.Grimes Acosmium cardenasii H.S.Irwin \& Arroyo Albizia lebbeck (L.) Benth.

Amburana cearensis (Allemão) A.C.Sm.

2 Anadenanthera colubrina (Vell.) Brenan Anadenanthera peregrina (L.) Speg.

Andira anthelmia (Vell.) Benth.

Andira cordata Arroyo ex R.T.Penn. \& H.C.Lima

1,2 Andira cujabensis Benth.

Andira fraxinifolia Benth.

Andira humilis Mart. ex Benth.

Andira inermis (W.Wright) DC.

Andira legalis (Vell.) Toledo

Andira nitida Mart. ex Benth.

Andira surinamensis (Bondt) Splitg. ex Amshoff

1,2 Andira vermifuga (Mart.) Benth.

Apuleia leiocarpa (Vogel) J.F.Macbr.

Bauhinia cheilantha (Bong.) Steud.

Bauhinia cupulata Benth.

Bauhinia grandifolia (Bong.) Steud.

Bauhinia longifolia (Bong.) Steud.

Bauhinia membranacea Benth.

Bauhinia pentandra (Bong.) Vogel ex Steud.

Bauhinia pulchella Benth.

2 Bauhinia rufa (Bong.) Steud.

Bauhinia ungulata L.

1,2 Bowdichia virgilioides Kunth

Calliandra foliolosa Benth.

Cassia ferruginea (Schrad.) Schrad. ex DC.

Cenostigma macrophyllum Tul.

Cenostigma tocantinum Ducke

Centrolobium tomentosum Guillem. ex Benth.

Chamaecrista apoucouita (Aubl.) H.S.Irwin \& Barneby

Chamaecrista dentata (Vogel) H.S.Irwin \& Barneby

Chamaecrista multiseta (Benth.) H.S.Irwin \& Barneby

Chamaecrista orbiculata (Benth.) H.S.Irwin \& Barneby

Chloroleucon acacioides (Ducke) Barneby \& J.W.Grimes

Chloroleucon mangense (Jacq.) Britton \& Rose

Copaifera coriacea Mart.

1,2 Copaifera langsdorffii Desf.

Copaifera martii Hayne

Copaifera oblongifolia Mart. ex Hayne

Lonchocarpus araripensis Benth.

Dalbergia brasiliensis Vogel

Dalbergia cearensis Ducke

1,2 Dalbergia miscolobium Benth.

Dialium guianense (Aubl.) Sandwith

Dimorphandra gardneriana Tul.

1,2 Dimorphandra mollis Benth.

1,2 Dipteryx alata Vogel

Dipteryx lacunifera Ducke

Diptychandra aurantiaca Tul.

Enterolobium contortisiliquum (Vell.) Morong

Enterolobium ellipticum Benth.

1,2 Enterolobium gummiferum (Mart.) J.F.Macbr.
$1 \quad 0.2 \% \quad \mathrm{ARB} / \mathrm{ARV}$

$20.3 \%$ ARB/ARV

$10.2 \%$ ARV

$10.2 \%$ ARV

$4 \quad 0.6 \%$ ARV

$68 \quad 10.9 \% \quad \mathrm{ARB} / \mathrm{ARV}$

$106 \quad 17.0 \% \quad \mathrm{ARB} / \mathrm{ARV}$

$6 \quad 1.0 \% \quad$ ARV

$25 \quad 4.0 \%$ ARV

$170 \quad 27.2 \% \quad$ ARV

$4 \quad 0.6 \%$ ARV

$31 \quad 5.0 \% \quad \mathrm{ARB} / \mathrm{ARV}$

$10 \quad 1.6 \%$ ARV

$6 \quad 1.0 \%$ ARV

$1 \quad 0.2 \% \quad \mathrm{ARB} / \mathrm{ARV}$

$2 \quad 0.3 \% \quad \mathrm{ARB} / \mathrm{ARV}$

$254 \quad 40.6 \%$ ARV

$213.4 \%$ ARV

$1 \quad 0.2 \% \quad$ ARB/ARV

$10.2 \% \quad \mathrm{ARB} / \mathrm{ARV}$

$1 \quad 0.2 \% \quad \mathrm{ARB} / \mathrm{ARV}$

$7 \quad 1.1 \% \quad$ ARB/ARV

$2 \quad 0.3 \% \quad \mathrm{ARB} / \mathrm{ARV}$

$10.2 \% \quad \mathrm{ARB} / \mathrm{ARV}$

$26 \quad 4.2 \% \quad \mathrm{ARB} / \mathrm{ARV}$

115 18.4\% SUB/ARB/ARV

$213.4 \% \quad \mathrm{SUB} / \mathrm{ARB} / \mathrm{ARV}$

$46474.2 \%$ ARB/ARV

$10.2 \% \quad \mathrm{ARB} / \mathrm{ARV}$

$10.2 \%$ ARV

$32 \quad 5.1 \% \quad \mathrm{ARB} / \mathrm{ARV}$

$10.2 \%$ ARV

$10.2 \%$ ARV

$10.2 \%$ ARV

$1 \quad 0.2 \% \quad \mathrm{ARB} / \mathrm{ARV}$

$1 \quad 0.2 \% \quad \mathrm{ARB} / \mathrm{ARV}$

$34 \quad 5.4 \%$ SUB/ARB/ARV

$20.3 \%$ ARV

$10.2 \%$ ARV

$9 \quad 1.4 \% \quad$ ARB/ARV

$276 \quad 44.2 \%$ ARV

$82 \quad 13.1 \% \quad$ ARB/ARV

$11 \quad 1.8 \% \quad \mathrm{ARB} / \mathrm{ARV}$

$30.5 \%$ ARV

$30.5 \%$ ARV

$1 \quad 0.2 \%$ ARV

$278 \quad 44.5 \%$ ARV

$1 \quad 0.2 \%$ ARV

$57 \quad 9.1 \%$ ARV

$411 \quad 65.8 \%$ ARV

$176 \quad 28.2 \%$ ARV

$10.2 \%$ ARV

$6911.0 \%$ ARV

$17 \quad 2.7 \%$ ARV

$6 \quad 1.0 \%$ ARV

$21033.6 \%$ ARV 
Fabaceae

Fabaceae

Fabaceae

Fabaceae

Fabaceae

Fabaceae

Fabaceae

Fabaceae

Fabaceae

Fabaceae

Fabaceae

Fabaceae

Fabaceae

Fabaceae

Fabaceae

Fabaceae

Fabaceae

Fabaceae

Fabaceae

Fabaceae

Fabaceae

Fabaceae

Fabaceae

Fabaceae

Fabaceae

Fabaceae

Fabaceae

Fabaceae

Fabaceae

Fabaceae

Fabaceae

Fabaceae

Fabaceae

Fabaceae

Fabaceae

Fabaceae

Fabaceae

Fabaceae

Fabaceae

Fabaceae

Fabaceae

Fabaceae

Fabaceae

Fabaceae

Fabaceae

Fabaceae

Fabaceae

Fabaceae

Fabaceae

Fabaceae

Fabaceae

Fabaceae

Fabaceae

Fabaceae

Fabaceae

Fabaceae
Enterolobium timbouva Mart.

Erythrina falcata Benth.

Erythrina velutina Willd.

Erythrina verna Vell.

Hymenaea courbaril L.

Hymenaea eriogyne Benth.

Hymenaea maranhensis Y.T.Lee \& Langenh.

Hymenaea martiana Hayne

1,2 Hymenaea stigonocarpa Mart. ex Hayne

Hymenolobium heringeranum Rizzini

Inga alba (Sw.) Willd.

Inga cylindrica (Vell.) Mart.

Inga edulis Mart.

Inga heterophylla Willd.

Inga laurina (Sw.) Willd.

Inga marginata Willd.

Inga striata Benth.

Inga thibaudiana DC.

Inga vera Willd.

1,2 Leptolobium dasycarpum Vogel

Leptolobium elegans Vogel

Leptolobium glaziovianum (Harms) Sch.Rodr. \& A.M.G.Azevedo

Leucochloron incuriale (Vell.) Barneby \& J.W.Grimes

Libidibia ferrea (Mart. ex Tul.) L.P.Queiroz

Luetzelburgia auriculata (Allemão) Ducke

1,2 Machaerium acutifolium Vogel

Machaerium amplum Benth.

Machaerium brasiliense Vogel

Machaerium eriocarpum Benth.

Machaerium hirtum (Vell.) Stellfeld

Machaerium nyctitans (Vell.) Benth.

1,2 Machaerium opacum Vogel

Machaerium scleroxylon Tul.

Machaerium stipitatum Vogel

Machaerium villosum Vogel

Martiodendron mediterraneum (Mart. ex Benth.) R.C.Koeppen

Martiodendron parviflorum (Amshoff) R.C.Koeppen

Mimosa acutistipula (Mart.) Benth.

Mimosa arenosa (Willd.) Poir.

Mimosa bimucronata (DC.) Kuntze

Mimosa caesalpiniifolia Benth.

Mimosa gemmulata Barneby

Mimosa laticifera Rizzini \& A.Mattos

Moldenhawera acuminata Afr.Fern. \& P.Bezerra

Ormosia arborea (Vell.) Harms

Parkia igneiflora Ducke

Parkia platycephala Benth.

Peltogyne confertiflora (Mart. ex Hayne) Benth.

Peltophorum dubium (Spreng.) Taub.

Piptadenia gonoacantha (Mart.) J.F.Macbr.

Piptadenia paniculata Benth.

Pityrocarpa moniliformis (Benth.) Luckow \& R.W.Jobson

1,2 Plathymenia reticulata Benth.

Platycyamus regnellii Benth.

Platymiscium floribundum Vogel

2 Platypodium elegans Vogel

\begin{tabular}{|c|c|c|}
\hline 1 & $0.2 \%$ & ARV \\
\hline 1 & $0.2 \%$ & ARV \\
\hline 1 & $0.2 \%$ & ARV \\
\hline 1 & $0.2 \%$ & ARV \\
\hline 70 & $11.2 \%$ & ARV \\
\hline 7 & $1.1 \%$ & $\mathrm{ARB} / \mathrm{ARV}$ \\
\hline 13 & $2.1 \%$ & ARV \\
\hline 5 & $0.8 \%$ & ARV \\
\hline 433 & $69.3 \%$ & ARV \\
\hline 4 & $0.6 \%$ & ARV \\
\hline 1 & $0.2 \%$ & ARV \\
\hline 3 & $0.5 \%$ & ARV \\
\hline 1 & $0.2 \%$ & ARV \\
\hline 2 & $0.3 \%$ & ARV \\
\hline 5 & $0.8 \%$ & ARV \\
\hline 5 & $0.8 \%$ & ARV \\
\hline 1 & $0.2 \%$ & ARV \\
\hline 1 & $0.2 \%$ & ARV \\
\hline 8 & $1.3 \%$ & ARV \\
\hline 339 & $54.2 \%$ & ARV \\
\hline 121 & $19.4 \%$ & ARV \\
\hline 1 & $0.2 \%$ & ARV \\
\hline 5 & $0.8 \%$ & ARV \\
\hline 5 & $0.8 \%$ & ARV \\
\hline 10 & $1.6 \%$ & ARV \\
\hline 338 & $54.1 \%$ & ARV \\
\hline 2 & $0.3 \%$ & LIA/ARB/ARV \\
\hline 3 & $0.5 \%$ & LIA/ARB/ARV \\
\hline 2 & $0.3 \%$ & ARV \\
\hline 27 & $4.3 \%$ & ARV \\
\hline 1 & $0.2 \%$ & ARV \\
\hline 188 & $30.1 \%$ & ARV \\
\hline 7 & $1.1 \%$ & ARV \\
\hline 4 & $0.6 \%$ & ARV \\
\hline 22 & $3.5 \%$ & ARV \\
\hline 14 & $2.2 \%$ & ARV \\
\hline 1 & $0.2 \%$ & ARV \\
\hline 4 & $0.6 \%$ & $\mathrm{ARB} / \mathrm{ARV}$ \\
\hline 1 & $0.2 \%$ & ARB/ARV \\
\hline 1 & $0.2 \%$ & ARB/ARV \\
\hline 4 & $0.6 \%$ & ARB/ARV \\
\hline 1 & $0.2 \%$ & $\mathrm{ARB} / \mathrm{ARV}$ \\
\hline 29 & $4.6 \%$ & $\mathrm{ARB} / \mathrm{ARV}$ \\
\hline 1 & $0.2 \%$ & ARV \\
\hline 3 & $0.5 \%$ & ARV \\
\hline 1 & $0.2 \%$ & ARV \\
\hline 68 & $10.9 \%$ & ARV \\
\hline 54 & $8.6 \%$ & ARV \\
\hline 4 & $0.6 \%$ & ARV \\
\hline 7 & $1.1 \%$ & ARV \\
\hline 1 & $0.2 \%$ & ARV \\
\hline 6 & $1.0 \%$ & ARB/ARV \\
\hline 320 & $51.2 \%$ & $\mathrm{ARV}$ \\
\hline 2 & $0.3 \%$ & ARV \\
\hline 5 & $0.8 \%$ & ARV \\
\hline 106 & $17.0 \%$ & ARV \\
\hline
\end{tabular}


Fabaceae

Fabaceae

Fabaceae

Fabaceae

Fabaceae

Fabaceae

Fabaceae

Fabaceae

Fabaceae

Fabaceae

Fabaceae

Fabaceae

Fabaceae

Fabaceae

Fabaceae

Fabaceae

Fabaceae

Fabaceae

Fabaceae

Fabaceae

Fabaceae

Fabaceae

Fabaceae

Fabaceae

Fabaceae

Fabaceae

Fabaceae

Fabaceae

Fabaceae

Fabaceae

Fabaceae

Fabaceae

Fabaceae

Fabaceae

Fabaceae

Humiriaceae

Humiriaceae

Hypericaceae

Hypericaceae

Hypericaceae

Icacinaceae

Lacistemataceae

Lacistemataceae

Lamiaceae

Lamiaceae

Lamiaceae

Lamiaceae

Lamiaceae

Lamiaceae

Lamiaceae

Lamiaceae

Lamiaceae

Lamiaceae

Lamiaceae

Lamiaceae

Lamiaceae
Poincianella bracteosa (Tul.) L.P.Queiroz

Poincianella pyramidalis (Tul.) L.P.Queiroz

Pterocarpus villosus (Mart. ex Benth.) Benth.

2 Pterodon emarginatus Vogel

1,2 Pterodon pubescens (Benth.) Benth.

Pterogyne nitens Tul.

Samanea saman (Jacq.) Merr.

Samanea tubulosa (Benth.) Barneby \& J.W.Grimes

Senegalia polyphylla (DC.) Britton \& Rose

Senna acuruensis (Benth.) H.S.Irwin \& Barneby

Senna alata (L.) Roxb.

Senna bicapsularis (L.) Roxb.

Senna cana (Nees \& Mart.) H.S.Irwin \& Barneby

Senna cearensis Afr.Fern.

Senna georgica H.S.Irwin \& Barneby

Senna macranthera (DC. ex Collad.) H.S.Irwin \& Barneby

Senna pendula (Humb.\& Bonpl.ex Willd.) H.S.Irwin \& Barneby

Senna silvestris (Vell.) H.S.Irwin \& Barneby

Senna spectabilis (DC.) H.S.Irwin \& Barneby

Senna trachypus (Benth.) H.S.Irwin \& Barneby

1,2 Stryphnodendron adstringens (Mart.) Coville

Stryphnodendron coriaceum Benth.

Stryphnodendron fissuratum E.M.O.Martins

Stryphnodendron guianense (Aubl.) Benth.

Stryphnodendron polyphyllum Mart.

1,2 Stryphnodendron rotundifolium Mart.

Swartzia apetala Raddi

Swartzia jorori Harms

1,2 Tachigali aurea Tul.

Tachigali rubiginosa (Mart. ex Tul.) Oliveira-Filho

1,2 Tachigali subvelutina (Benth.) Oliveira-Filho

Tachigali vulgaris L.G.Silva \& H.C.Lima

1,2 Vatairea macrocarpa (Benth.) Ducke

Zollernia ilicifolia (Brongn.) Vogel

Zygia inaequalis (Willd.) Pittier

Humiria balsamifera (Aubl.) A. St.-Hil.

Sacoglottis guianensis Benth.

Vismia brasiliensis Choisy

Vismia guianensis (Aubl.) Choisy

Vismia pentagyna (Spreng.) Ewan

1,2 Emmotum nitens (Benth.) Miers

Lacistema aggregatum (P.J.Bergius) Rusby

Lacistema hasslerianum Chodat

Aegiphila brachiata Vell.

Aegiphila integrifolia (Jacq.) Moldenke

1 Aegiphila verticillata Vell.

Cyanocephalus lippioides (Pohl ex Benth.) Harley \& J.F.B.Pastore

Hyptidendron canum (Pohl ex Benth.) Harley

Hyptis pachyphylla Epling

Hyptis suaveolens Poit.

Vitex cymosa Bertero ex Spreng.

Vitex flavens Kunth

Vitex megapotamica (Spreng.) Moldenke

Vitex panshiniana Moldenke

Vitex polygama Cham.

Vitex schaueriana Moldenke

$\begin{array}{rrl}2 & 0.3 \% & \text { ARB/ARV } \\ 2 & 0.3 \% & \text { ARB/ARV } \\ 1 & 0.2 \% & \text { ARV } \\ 122 & 19.5 \% & \text { ARV } \\ 164 & 26.2 \% & \text { ARV } \\ 6 & 1.0 \% & \text { ARV } \\ 1 & 0.2 \% & \text { ARV } \\ 3 & 0.5 \% & \text { ARV } \\ 12 & 1.9 \% & \text { ARB/ARV } \\ 3 & 0.5 \% & \text { ARB/ARV } \\ 1 & 0.2 \% & \text { SUB/ARB/ARV } \\ 1 & 0.2 \% & \text { ARB/ARV } \\ 3 & 0.5 \% & \text { ARB/ARV } \\ 1 & 0.2 \% & \text { ARB/ARV } \\ 1 & 0.2 \% & \text { ARB/ARV } \\ 10 & 1.6 \% & \text { ARB/ARV } \\ 4 & 0.6 \% & \text { LIA/ARB/ARV } \\ 26 & 4.2 \% & \text { SUB/ARB/ARV } \\ 2 & 0.3 \% & \text { ARV } \\ 1 & 0.2 \% & \text { ARB/ARV }\end{array}$

$23237.1 \%$ ARV

$62 \quad 9.9 \%$ ARV

$50.8 \%$ ARV

$1 \quad 0.2 \%$ ARV

$43 \quad 6.9 \% \quad$ ARB/ARV

$193 \quad 30.9 \%$ ARB/ARV

$2 \quad 0.3 \% \quad$ ARV

$1 \quad 0.2 \% \quad$ ARV

284 45.4\% ARV

$2 \quad 0.3 \%$ ARV

$300 \quad 48.0 \%$ ARV

$26 \quad 4.2 \%$ ARV

$34354.9 \%$ ARV

$1 \quad 0.2 \% \quad$ ARB/ARV

$1 \quad 0.2 \% \quad \mathrm{ARB} / \mathrm{ARV}$

$7 \quad 1.1 \% \quad \mathrm{ARB} / \mathrm{ARV}$

$4 \quad 0.6 \%$ ARV

$2 \quad 0.3 \% \quad \mathrm{ARB} / \mathrm{ARV}$

$26 \quad 4.2 \% \quad$ ARB/ARV

$20.3 \%$ ARV

$257 \quad 41.1 \% \quad$ ARB/ARV

$17 \quad 2.7 \% \quad$ ARB/ARV

$27 \quad 4.3 \% \quad \mathrm{ARB} / \mathrm{ARV}$

$1 \quad 0.2 \% \quad \mathrm{ARB} / \mathrm{ARV}$

$12 \quad 1.9 \% \quad \mathrm{ARB} / \mathrm{ARV}$

$188 \quad 30.1 \%$ SUB/ARB/ARV

$10.2 \% \quad$ SUB/ARV

$46 \quad 7.4 \% \quad$ ARB/ARV

$20.3 \% \quad$ ARB/ARV

$5 \quad 0.8 \% \quad \mathrm{ARB} / \mathrm{ARV}$

$152.4 \%$ ARV

$3 \quad 0.5 \% \quad$ ARV

$10.2 \% \quad$ ARB/ARV

$3 \quad 0.5 \% \quad$ ARV

$40 \quad 6.4 \% \quad$ ARB/ARV

$10.2 \%$ ARV 
Lamiaceae

Lauraceae

Lauraceae

Lauraceae

Lauraceae

Lauraceae

Lauraceae

Lauraceae

Lauraceae

Lauraceae

Lauraceae

Lauraceae

Lauraceae

Lauraceae

Lauraceae

Lauraceae

Lauraceae

Lauraceae

Lauraceae

Lauraceae

Lauraceae

Lauraceae

Lauraceae

Lauraceae

Lauraceae

Lauraceae

Lauraceae

Lauraceae

Lauraceae

Lauraceae

Lauraceae

Lauraceae

Lauraceae

Lauraceae

Lauraceae

Lauraceae

Lauraceae

Lauraceae

Lauraceae

Lauraceae

Lecythidaceae

Lecythidaceae

Lecythidaceae

Lecythidaceae

Loganiaceae

Loganiaceae

Loganiaceae

Lythraceae

Lythraceae

Lythraceae

Lythraceae

Lythraceae

Malpighiaceae

Malpighiaceae

Malpighiaceae

Malpighiaceae
Vitex schomburgkiana Schauer

Aiouea trinervis Meisn.

Aniba heringeri Vattimo-Gil

Cinnamomum erythropus (Nees \& Mart.) Kosterm.

Cinnamomum sellowianum (Nees \& Mart.) Kosterm.

Endlicheria paniculata (Spreng.) J.F.Macbr.

Mezilaurus crassiramea (Meisn.) Taub.

Mezilaurus lindaviana Schwacke \& Mez

Nectandra cissiflora Nees

Nectandra cuspidata Nees

Nectandra hihua (Ruiz \& Pav.) Rohwer

Nectandra lanceolata Nees

Nectandra megapotamica (Spreng.) Mez

Nectandra membranacea (Sw.) Griseb.

Nectandra reticulata (Ruiz \& Pav.) Mez

Ocotea aciphylla (Nees \& Mart.) Mez

Ocotea acutifolia (Nees) Mez

Ocotea bicolor Vattimo?Gil

Ocotea canaliculata ( Rich.) Mez

Ocotea cernua (Nees) Mez

Ocotea corymbosa (Meisn.) Mez

Ocotea diospyrifolia (Meisn.) Mez

Ocotea felix Coe-Teix.

Ocotea glaucina (Meisn.) Mez

Ocotea lancifolia (Schott) Mez

Ocotea minarum (Nees \& Mart.) Mez

Ocotea nitida (Meisn.) Rohwer

Ocotea pomaderroides (Meisn.) Mez

Ocotea pulchella (Nees \& Mart.) Mez

Ocotea spixiana (Nees) Mez

Ocotea tristis (Nees \& Mart.) Mez

Ocotea velloziana (Meisn.) Mez

Ocotea velutina (Nees) Rohwer

Persea alba Nees \& Mart.

Persea americana Mill.

Persea fusca Mez

Persea major (Meisn.) L.E.Kopp

Persea splendens Meisn.

Persea venosa Nees \& Mart.

Persea willdenovii Kosterm.

Cariniana estrellensis (Raddi) Kuntze

Eschweilera coriacea (DC.)

Eschweilera nana (O.Berg) Miers

Lecythis lurida (Miers) S.A.Mori

Antonia ovata Pohl

Bonyunia antoniifolia Progel

1,2 Strychnos pseudoquina A.St.-Hil.

Diplusodon virgatus Pohl var. virgatus

Lafoensia densiflora Pohl

1,2 Lafoensia pacari A.St.-Hil.

1 Lafoensia vandelliana subsp. replicata (Pohl) Lourteig

2 Physocalymma scaberrimum Pohl

Banisteriopsis latifolia (A.Juss.) B.Gates

Bunchosia armeniaca (Cav.) DC.

Bunchosia paraguariensis Nied.

Byrsonima affinis W.R.Anderson
$20.3 \% \quad \mathrm{ARV}$

$10 \quad 1.6 \% \quad \mathrm{ARB} / \mathrm{ARV}$

$1 \quad 0.2 \% \quad$ ARB/ARV

$20.3 \%$ ARV

$30.5 \%$ ARV

$3 \quad 0.5 \% \quad$ ARB/ARV

$53 \quad 8.5 \% \quad$ ARB/ARV

$1 \quad 0.2 \% \quad$ ARB/ARV

$10.2 \%$ ARV

$5 \quad 0.8 \%$ ARV

$1 \quad 0.2 \%$ ARV

$4 \quad 0.6 \%$ ARV

$20.3 \%$ ARV

$10.2 \%$ ARV

$10.2 \%$ ARV

$6 \quad 1.0 \%$ ARV

$7 \quad 1.1 \%$ ARV

$10.2 \%$ ARV

$10.2 \%$ ARV

$20.3 \%$ ARV

$416.6 \%$ ARV

$7 \quad 1.1 \%$ ARV

$10.2 \%$ ARV

$1 \quad 0.2 \% \quad$ ARB/ARV

$2 \quad 0.3 \% \quad$ ARV

$20 \quad 3.2 \% \quad$ ARB/ARV

$1 \quad 0.2 \% \quad \mathrm{ARB} / \mathrm{ARV}$

$9 \quad 1.4 \%$ ARV

$59 \quad 9.4 \%$ ARV

$132.1 \%$ ARV

$4 \quad 0.6 \%$ ARB/ARV

$4 \quad 0.6 \%$ ARV

$1 \quad 0.2 \% \quad$ ARV

$20.3 \%$ ARV

$10.2 \%$ ARV

$10.2 \%$ ARV

$20.3 \%$ ARV

$10.2 \%$ ARV

$30.5 \%$ ARV

$11 \quad 1.8 \%$ ARV

$4 \quad 0.6 \%$ ARV

$1 \quad 0.2 \% \quad$ ARV

$54 \quad 8.6 \% \quad$ ARB/ARV

$2 \quad 0.3 \%$ ARV

$100 \quad 16.0 \%$ ARV

$1 \quad 0.2 \%$ ARV

$239 \quad 38.2 \% \quad$ ARB/ARV

$10 \quad 1.6 \% \quad$ SUB/ARB/ARV

$5 \quad 0.8 \% \quad \mathrm{ARB} / \mathrm{ARV}$

$185 \quad 29.6 \%$ ARV

$235 \quad 37.6 \%$ ARV

$7211.5 \%$ ARV

$34 \quad 5.4 \%$ ARV

$10.2 \% \quad \mathrm{ARB} / \mathrm{ARV}$

$10.2 \%$ ARV

$30.5 \%$ ARV 
Malpighiaceae

Malpighiaceae

Malpighiaceae

Malpighiaceae

Malpighiaceae

Malpighiaceae

Malpighiaceae

Malpighiaceae

Malpighiaceae

Malpighiaceae

Malpighiaceae

Malpighiaceae

Malpighiaceae

Malpighiaceae

Malpighiaceae

Malpighiaceae

Malpighiaceae

Malpighiaceae

Malpighiaceae

Malvaceae

Malvaceae

Malvaceae

Malvaceae

Malvaceae

Malvaceae

Malvaceae

Malvaceae

Malvaceae

Malvaceae

Malvaceae

Malvaceae

Malvaceae

Malvaceae

Malvaceae

Malvaceae

Malvaceae

Malvaceae

Marcgraviaceae

Marcgraviaceae

Melastomataceae

Melastomataceae

Melastomataceae

Melastomataceae

Melastomataceae

Melastomataceae

Melastomataceae

Melastomataceae

Melastomataceae

Melastomataceae

Melastomataceae

Melastomataceae

Melastomataceae

Melastomataceae

Melastomataceae

Melastomataceae

Melastomataceae
Byrsonima arthropoda A.Juss.

Byrsonima clausseniana A.Juss.

1,2 Byrsonima coccolobifolia Kunth

1 Byrsonima crassifolia (L.) Kunth

Byrsonima cydoniifolia A.Juss.

Byrsonima gardneriana A.Juss.

Byrsonima lancifolia A.Juss.

Byrsonima laxiflora Griseb.

Byrsonima linguifera Cuatrec.

1,2 Byrsonima pachyphylla A.Juss.

Byrsonima punctulata A.Juss.

Byrsonima rotunda Griseb.

Byrsonima salzmanniana A.Juss.

Byrsonima schomburgkiana Benth.

Byrsonima sericea DC.

Byrsonima stipulacea A.Juss.

Byrsonima vacciniifolia A.Juss.

1,2 Byrsonima verbascifolia (L.) DC.

1,2 Heteropterys byrsonimiifolia A.Juss. Apeiba tibourbou Aubl.

1,2 Eriotheca gracilipes (K.Schum.) A.Robyns Eriotheca parvifolia (Mart. \& Zucc.) A.Robyns

1,2 Eriotheca pubescens (Mart. \& Zucc.) Schott \& Endl. Eriotheca roseorum (Cuatrec.) A.Robyns

2 Guazuma ulmifolia Lam.

Luehea candicans Mart. \& Zucc.

Luehea divaricata Mart. \& Zucc.

Luehea grandiflora Mart. \& Zucc.

Luehea speciosa Willd.

Mollia burchellii Sprague

Pseudobombax grandiflorum (Cav.) A.Robyns

1,2 Pseudobombax longiflorum (Mart. \& Zucc.) A.Robyns

Pseudobombax marginatum (A.St.-Hil.) A. Robyns

Pseudobombax minimum Carv.?Sobr. \& L.P. Queiroz

2 Pseudobombax tomentosum (Mart. \& Zucc.) A.Robyns

Sterculia apetala (Jacq.) H.Karst.

Sterculia striata A.St.-Hil. \& Naudin

Norantea guianensis Aubl.

Schwartzia adamantium (Cambess.) Bedell ex Gir.?Cañas

Leandra acutiflora (Naudin) Cogn.

Leandra aurea (Cham.) Cogn.

Leandra lancifolia Cogn.

Leandra melastomoides Raddi

Macairea radula (Bonpl.) DC.

1,2 Miconia albicans (Sw.) Triana

Miconia chartacea Triana

Miconia cinerascens Miq.

Miconia cinnamomifolia (DC.) Naudin

Miconia cubatanensis Hoehne

Miconia cuspidata Naudin

Miconia elegans Cogn.

2 Miconia ferruginata DC.

Miconia gratissima Benth. ex Triana

Miconia hyemalis A.St.-Hil. \& Naudin

Miconia ibaguensis (Bonpl.) Triana

Miconia ligustroides (DC.) Naudin

\begin{tabular}{|c|c|c|}
\hline 4 & $0.6 \%$ & ARV \\
\hline 4 & $0.6 \%$ & $\mathrm{ARB} / \mathrm{ARV}$ \\
\hline 422 & $67.5 \%$ & $\mathrm{ARB} / \mathrm{ARV}$ \\
\hline 143 & $22.9 \%$ & ARV \\
\hline 38 & $6.1 \%$ & $\mathrm{ARB} / \mathrm{ARV}$ \\
\hline 1 & $0.2 \%$ & $\mathrm{ARB} / \mathrm{ARV}$ \\
\hline 5 & $0.8 \%$ & $\mathrm{ARB} / \mathrm{ARV}$ \\
\hline 12 & $1.9 \%$ & ARV \\
\hline 8 & $1.3 \%$ & ARV \\
\hline 299 & $47.8 \%$ & $\mathrm{ARB} / \mathrm{ARV}$ \\
\hline 1 & $0.2 \%$ & $\mathrm{ARB} / \mathrm{ARV}$ \\
\hline 1 & $0.2 \%$ & $\mathrm{ARB} / \mathrm{ARV}$ \\
\hline 3 & $0.5 \%$ & ARV \\
\hline 2 & $0.3 \%$ & $\mathrm{ARB} / \mathrm{ARV}$ \\
\hline 52 & $8.3 \%$ & $\mathrm{ARB} / \mathrm{ARV}$ \\
\hline 6 & $1.0 \%$ & ARV \\
\hline 3 & $0.5 \%$ & $\mathrm{ARB} / \mathrm{ARV}$ \\
\hline 303 & $48.5 \%$ & ARB/ARV \\
\hline 194 & $31.0 \%$ & LIA/ARB/ARV \\
\hline 31 & $5.0 \%$ & ARV \\
\hline 298 & $47.7 \%$ & ARV \\
\hline 28 & $4.5 \%$ & ARV \\
\hline 126 & $20.2 \%$ & ARV \\
\hline 1 & $0.2 \%$ & ARV \\
\hline 114 & $18.2 \%$ & ARV \\
\hline 26 & $4.2 \%$ & ARV \\
\hline 57 & $9.1 \%$ & ARV \\
\hline 45 & $7.2 \%$ & ARV \\
\hline 3 & $0.5 \%$ & $\mathrm{ARB} / \mathrm{ARV}$ \\
\hline 10 & $1.6 \%$ & ARV \\
\hline 2 & $0.3 \%$ & ARV \\
\hline 251 & $40.2 \%$ & ARV \\
\hline 3 & $0.5 \%$ & ARV \\
\hline 3 & $0.5 \%$ & ARV \\
\hline 118 & $18.9 \%$ & ARV \\
\hline 4 & $0.6 \%$ & ARV \\
\hline 30 & $4.8 \%$ & ARV \\
\hline 17 & $2.7 \%$ & LIA/ARB/ARV \\
\hline 22 & $3.5 \%$ & LIA/ARB/ARV \\
\hline 3 & $0.5 \%$ & $\mathrm{ARB} / \mathrm{ARV}$ \\
\hline 9 & $1.4 \%$ & $\mathrm{ARB} / \mathrm{ARV}$ \\
\hline 3 & $0.5 \%$ & $\mathrm{ARB} / \mathrm{ARV}$ \\
\hline 1 & $0.2 \%$ & $\mathrm{ARB} / \mathrm{ARV}$ \\
\hline 10 & $1.6 \%$ & SUB/ARB/ARV \\
\hline 299 & $47.8 \%$ & $\mathrm{ARB} / \mathrm{ARV}$ \\
\hline 2 & $0.3 \%$ & ARV \\
\hline 6 & $1.0 \%$ & $\mathrm{ARB} / \mathrm{ARV}$ \\
\hline 3 & $0.5 \%$ & $\mathrm{ARB} / \mathrm{ARV}$ \\
\hline 1 & $0.2 \%$ & ARV \\
\hline 6 & $1.0 \%$ & ARV \\
\hline 1 & $0.2 \%$ & $\mathrm{ARB} / \mathrm{ARV}$ \\
\hline 102 & $16.3 \%$ & $\mathrm{ARB} / \mathrm{ARV}$ \\
\hline 1 & $0.2 \%$ & $\mathrm{ARB} / \mathrm{ARV}$ \\
\hline 5 & $0.8 \%$ & $\mathrm{ARB} / \mathrm{ARV}$ \\
\hline 2 & $0.3 \%$ & $\mathrm{ARB} / \mathrm{ARV}$ \\
\hline 58 & $9.3 \%$ & $\mathrm{ARB} / \mathrm{ARV}$ \\
\hline
\end{tabular}


Melastomataceae

Melastomataceae

Melastomataceae

Melastomataceae

Melastomataceae

Melastomataceae

Melastomataceae

Melastomataceae

Melastomataceae

Melastomataceae

Melastomataceae

Melastomataceae

Melastomataceae

Melastomataceae

Melastomataceae

Melastomataceae

Melastomataceae

Melastomataceae

Melastomataceae

Melastomataceae

Melastomataceae

Melastomataceae

Melastomataceae

Melastomataceae

Melastomataceae

Melastomataceae

Melastomataceae

Melastomataceae

Melastomataceae

Meliaceae

Meliaceae

Meliaceae

Meliaceae

Meliaceae

Meliaceae

Meliaceae

Meliaceae

Meliaceae

Meliaceae

Menispermaceae

Moracea

Moraceae

Moraceae

Moraceae

Moraceae

Moraceae

Moraceae

Moraceae

Moraceae

Moraceae

Moraceae

Moraceae

Moraceae

Moraceae

Moraceae

Moraceae
Miconia minutiflora (Bonpl.) DC

Miconia nervosa (Sm.) Triana

Miconia paucidens DC.

Miconia pepericarpa DC.

Miconia petropolitana Cogn.

Miconia polyandra Gardner

Miconia prasina (Sw.) DC.

Miconia punctata (Desr.) DC.

Miconia pyrifolia Naudin

Miconia rubiginosa (Bonpl.) DC.

Miconia sclerophylla Triana

Miconia sellowiana Naudin

Miconia theaezans (Bonpl.) Cogn.

Miconia tomentosa (Rich.) D.Don

Mouriri acutiflora Naudin

Mouriri apiranga Spruce ex Triana

Mouriri cearensis Huber

1,2 Mouriri elliptica Mart.

Mouriri glazioviana Cogn.

Mouriri guianensis Aubl.

2 Mouriri pusa Gardner

Tibouchina aspera Aubl.

Tibouchina candolleana (Mart. ex DC.) Cogn.

Tibouchina granulosa (Desr.) Cogn.

Tibouchina papyrus (Pohl) Toledo

Tibouchina sellowiana Cogn.

Tibouchina stenocarpa (Schrank \& Mart.ex DC.) Cogn.

Trembleya laniflora (D.Don) Cogn.

Trembleya parviflora (D.Don) Cogn.

Cabralea canjerana (Vell.) Mart.

Cedrela fissilis Vell.

Guarea kunthiana A.Juss.

Guarea macrophylla Vahl

Trichilia catigua A.Juss.

Trichilia clausseni C.DC.

Trichilia elegans A.Juss.

Trichilia hirta L.

Trichilia micrantha Benth.

Trichilia pallida Sw.

Abuta grandifolia (Mart.) Sandwith

Sorocea klotzschiana Baill.

1,2 Brosimum gaudichaudii Trécul

Brosimum lactescens (S.Moore) C.C.Berg

Ficus americana Aubl.

Ficus arpazusa Casar.

Ficus catappifolia Kunth \& C.D.Bouché

Ficus citrifolia Mill.

Ficus elliotiana S.Moore

Ficus enormis Mart. ex Miq.

Ficus eximia Schott

Ficus gomelleira Kunth

Ficus guaranitica Chodat

Ficus insipida Willd.

Ficus obtusifolia Kunth

Ficus pertusa L.f.

Maclura tinctoria (L.) D.Don ex Steud.
$1 \quad 0.2 \% \quad$ ARV

$2 \quad 0.3 \% \quad \mathrm{ARB} / \mathrm{ARV}$

$18 \quad 2.9 \% \quad$ ARB/ARV

$152.4 \% \quad \mathrm{ARB} / \mathrm{ARV}$

$10.2 \% \quad \mathrm{ARB} / \mathrm{ARV}$

$20.3 \% \quad \mathrm{ARB} / \mathrm{ARV}$

$4 \quad 0.6 \% \quad \mathrm{ARB} / \mathrm{ARV}$

$1 \quad 0.2 \% \quad$ ARB/ARV

$2 \quad 0.3 \% \quad$ ARV

$73 \quad 11.7 \% \quad$ ARB/ARV

$1 \quad 0.2 \%$ ARV

$29 \quad 4.6 \%$ ARV

$5 \quad 0.8 \% \quad \mathrm{ARB} / \mathrm{ARV}$

$10.2 \% \quad$ ARB/ARV

$20.3 \% \quad$ ARV

$2 \quad 0.3 \% \quad \mathrm{ARB} / \mathrm{ARV}$

$1 \quad 0.2 \% \quad \mathrm{ARB} / \mathrm{ARV}$

$171 \quad 27.4 \%$ ARB/ARV

$3 \quad 0.5 \% \quad$ ARV

$6 \quad 1.0 \% \quad$ ARB/ARV

$108 \quad 17.3 \%$ ARB/ARV

$1 \quad 0.2 \% \quad$ ARV

$8 \quad 1.3 \% \quad \mathrm{ARB} / \mathrm{ARV}$

$1 \quad 0.2 \%$ ARV

$7 \quad 1.1 \%$ ARV

$20.3 \%$ ARV

$25 \quad 4.0 \%$ ARV

$1 \quad 0.2 \% \quad$ ARB/ARV

$132.1 \% \quad \mathrm{SUB} / \mathrm{ARB} / \mathrm{ARV}$

$28 \quad 4.5 \%$ ARV

$12 \quad 1.9 \%$ ARV

$1 \quad 0.2 \%$ ARV

$20.3 \%$ ARV

$20.3 \%$ ARV

$1 \quad 0.2 \% \quad$ ARV

$5 \quad 0.8 \% \quad$ ARB/ARV

$10.2 \% \quad$ ARV

$1 \quad 0.2 \% \quad$ ARV

$10 \quad 1.6 \%$ ARV

$152.4 \%$ LIA/ARB/ARV

$30.5 \% \quad$ ARB/ARV

$31350.1 \%$ ARV

$10.2 \%$ ARV

$4 \quad 0.6 \%$ ARV

$3 \quad 0.5 \%$ ARV

$4 \quad 0.6 \%$ ARV

$11 \quad 1.8 \%$ ARV

$1 \quad 0.2 \% \quad$ ARV

$6 \quad 1.0 \%$ ARV

$10.2 \%$ ARV

$8 \quad 1.3 \%$ ARV

$4 \quad 0.6 \%$ ARV

$30.5 \%$ ARV

$10.2 \%$ ARV

$30.5 \%$ ARV

$8 \quad 1.3 \% \quad$ ARV 


\begin{tabular}{|c|c|c|}
\hline Moraceae & & Pseudolmedia laevigata Trécul \\
\hline Moraceae & & Sorocea bonplandii (Baill.) W.C.Burger et al. \\
\hline Moraceae & & Sorocea guilleminiana Gaudich. \\
\hline Moraceae & & Sorocea saxicola Hassl. \\
\hline Moraceae & & Sorocea sprucei (Baill.) J.F.Macbr. \\
\hline Myristicaceae & & Virola gardneri (A.DC.) Warb. \\
\hline Myristicaceae & 1,2 & Virola sebifera Aubl. \\
\hline Myrtaceae & & Blepharocalyx salicifolius (Kunth) O.Berg \\
\hline Myrtaceae & & Calyptranthes clusiifolia O.Berg \\
\hline Myrtaceae & & Calyptranthes concinna DC. \\
\hline Myrtaceae & & Calyptranthes lucida Mart. ex DC. \\
\hline Myrtaceae & & Campomanesia aromatica (Aubl.) Griseb. \\
\hline Myrtaceae & & Campomanesia eugenioides (Cambess.) D.Legrand \\
\hline Myrtaceae & & Campomanesia grandiflora (Aubl.) Sagot \\
\hline Myrtaceae & & Campomanesia guaviroba (DC.) Kiaersk. \\
\hline Myrtaceae & & Campomanesia guazumifolia (Cambess.) O.Berg \\
\hline Myrtaceae & & Campomanesia pubescens (DC.) O.Berg \\
\hline Myrtaceae & & Campomanesia sessiliflora (O.Berg) Mattos \\
\hline Myrtaceae & & Campomanesia velutina (Cambess.) O.Berg \\
\hline Myrtaceae & & Campomanesia xanthocarpa (Mart.) O.Berg \\
\hline Myrtaceae & & Eugenia acutata Miq. \\
\hline Myrtaceae & & Eugenia aurata O.Berg \\
\hline Myrtaceae & & Eugenia biflora (L.) DC. \\
\hline Myrtaceae & & Eugenia brasiliensis Lam. \\
\hline Myrtaceae & & Eugenia cerasiflora Miq. \\
\hline Myrtaceae & 1,2 & Eugenia dysenterica DC. \\
\hline Myrtaceae & & Eugenia egensis DC. \\
\hline Myrtaceae & & Eugenia florida DC. \\
\hline Myrtaceae & & Eugenia francavilleana O.Berg \\
\hline Myrtaceae & & Eugenia hiemalis Cambess. \\
\hline Myrtaceae & & Eugenia inundata DC. \\
\hline Myrtaceae & & Eugenia involucrata DC. \\
\hline Myrtaceae & & Eugenia luschnathiana (O.Berg) Klotzsch ex B.D.Jacks. \\
\hline Myrtaceae & & Eugenia lutescens Cambess. \\
\hline Myrtaceae & & Eugenia myrcianthes Nied. \\
\hline Myrtaceae & & Eugenia neolaurifolia Sobral \\
\hline Myrtaceae & & Eugenia piloensis Cambess. \\
\hline Myrtaceae & & Eugenia pluriflora DC. \\
\hline Myrtaceae & & Eugenia pyriformis Cambess. \\
\hline Myrtaceae & & Eugenia sellowiana DC. \\
\hline Myrtaceae & & Eugenia sonderiana O.Berg \\
\hline Myrtaceae & & Eugenia stictopetala DC. \\
\hline Myrtaceae & & Miconia ferruginea (Desr.) DC. \\
\hline Myrtaceae & & Myrceugenia alpigena (DC.) Landrum \\
\hline Myrtaceae & & Myrceugenia bracteosa (DC.) D.Legrand \& Kausel \\
\hline Myrtaceae & & Myrceugenia hatschbachii Landrum \\
\hline Myrtaceae & & Myrceugenia myrcioides (Cambess.) O.Berg \\
\hline Myrtaceae & & Myrceugenia oxysepala (Burret) D.Legrand \& Kausel \\
\hline Myrtaceae & & Myrcia bracteata (Rich.) DC. \\
\hline Myrtaceae & & Myrcia cordiifolia DC. \\
\hline Myrtaceae & & Myrcia cuprea (O.Berg) Kiaersk. \\
\hline Myrtaceae & & Myrcia fenzliana O.Berg \\
\hline Myrtaceae & & Myrcia glabra (O.Berg) D.Legrand \\
\hline Myrtaceae & 1 & Myrcia guianensis (Aubl.) DC. \\
\hline Myrtaceae & & Myrcia hartwegiana (O.Berg) Kiaersk. \\
\hline Myrtaceae & & Myrcia hebepetala DC. \\
\hline
\end{tabular}

\begin{tabular}{|c|c|c|}
\hline 1 & $0.2 \%$ & ARV \\
\hline 2 & $0.3 \%$ & $\mathrm{ARB} / \mathrm{ARV}$ \\
\hline 6 & $1.0 \%$ & $\mathrm{ARB} / \mathrm{ARV}$ \\
\hline 2 & $0.3 \%$ & $\mathrm{ARB} / \mathrm{ARV}$ \\
\hline 2 & $0.3 \%$ & $\mathrm{ARB} / \mathrm{ARV}$ \\
\hline 1 & $0.2 \%$ & ARV \\
\hline 131 & $21.0 \%$ & $\mathrm{ARB} / \mathrm{ARV}$ \\
\hline 67 & $10.7 \%$ & ARV \\
\hline 3 & $0.5 \%$ & ARV \\
\hline 3 & $0.5 \%$ & ARV \\
\hline 1 & $0.2 \%$ & ARV \\
\hline 2 & $0.3 \%$ & ARV \\
\hline 5 & $0.8 \%$ & $\mathrm{ARB} / \mathrm{ARV}$ \\
\hline 5 & $0.8 \%$ & ARV \\
\hline 4 & $0.6 \%$ & ARV \\
\hline 3 & $0.5 \%$ & ARV \\
\hline 42 & $6.7 \%$ & $\mathrm{ARB} / \mathrm{ARV}$ \\
\hline 1 & $0.2 \%$ & ARB/ARV \\
\hline 10 & $1.6 \%$ & ARV \\
\hline 5 & $0.8 \%$ & ARV \\
\hline 1 & $0.2 \%$ & ARV \\
\hline 109 & $17.4 \%$ & $\mathrm{ARB} / \mathrm{ARV}$ \\
\hline 11 & $1.8 \%$ & ARV \\
\hline 1 & $0.2 \%$ & ARV \\
\hline 1 & $0.2 \%$ & ARV \\
\hline 215 & $34.4 \%$ & $\mathrm{ARB} / \mathrm{ARV}$ \\
\hline 2 & $0.3 \%$ & ARV \\
\hline 14 & $2.2 \%$ & ARV \\
\hline 1 & $0.2 \%$ & ARV \\
\hline 19 & $3.0 \%$ & ARB/ARV \\
\hline 2 & $0.3 \%$ & $\mathrm{ARB} / \mathrm{ARV}$ \\
\hline 6 & $1.0 \%$ & ARV \\
\hline 1 & $0.2 \%$ & ARV \\
\hline 2 & $0.3 \%$ & ARB/ARV \\
\hline 3 & $0.5 \%$ & $\mathrm{ARB} / \mathrm{ARV}$ \\
\hline 1 & $0.2 \%$ & ARV \\
\hline 2 & $0.3 \%$ & $\mathrm{ARB} / \mathrm{ARV}$ \\
\hline 1 & $0.2 \%$ & ARV \\
\hline 6 & $1.0 \%$ & SUB/ARB/ARV \\
\hline 1 & $0.2 \%$ & $\mathrm{ARB} / \mathrm{ARV}$ \\
\hline 1 & $0.2 \%$ & $\mathrm{ARB} / \mathrm{ARV}$ \\
\hline 4 & $0.6 \%$ & $\mathrm{ARB} / \mathrm{ARV}$ \\
\hline 2 & $0.3 \%$ & $\mathrm{ARB} / \mathrm{ARV}$ \\
\hline 3 & $0.5 \%$ & ARV \\
\hline 1 & $0.2 \%$ & ARV \\
\hline 1 & $0.2 \%$ & ARV \\
\hline 1 & $0.2 \%$ & ARV \\
\hline 1 & $0.2 \%$ & $\mathrm{ARB} / \mathrm{ARV}$ \\
\hline 1 & $0.2 \%$ & $\mathrm{ARB} / \mathrm{ARV}$ \\
\hline 3 & $0.5 \%$ & ARV \\
\hline 3 & $0.5 \%$ & $\mathrm{ARB} / \mathrm{ARV}$ \\
\hline 17 & $2.7 \%$ & ARV \\
\hline 1 & $0.2 \%$ & ARV \\
\hline 156 & $25.0 \%$ & ARV \\
\hline 3 & $0.5 \%$ & ARV \\
\hline 1 & $0.2 \%$ & ARV \\
\hline
\end{tabular}




\begin{tabular}{|c|c|c|}
\hline Myrtaceae & & Myrcia laruotteana Cambess. \\
\hline Myrtaceae & & Myrcia multiflora (Lam.) DC. \\
\hline Myrtaceae & & Myrcia mutabilis (O.Berg) N.Silveira \\
\hline Myrtaceae & & Myrcia obovata (O.Berg) Nied. \\
\hline Myrtaceae & & Myrcia ochroides O. Berg \\
\hline Myrtaceae & & Myrcia palustris DC. \\
\hline Myrtaceae & & Myrcia pubipetala Miq. \\
\hline Myrtaceae & & Myrcia selloi (Spreng.) N.Silveira \\
\hline Myrtaceae & 1 & Myrcia splendens (Sw.) DC. \\
\hline Myrtaceae & & Myrcia subcordata DC. \\
\hline Myrtaceae & 1 & Myrcia tomentosa (Aubl.) DC. \\
\hline Myrtaceae & & Myrcia variabilis DC. \\
\hline Myrtaceae & & Myrcia venulosa DC. \\
\hline Myrtaceae & & Myrcia vestita DC. \\
\hline Myrtaceae & & Myrcianthes pungens (O.Berg) \\
\hline Myrtaceae & & Myrciaria cuspidata O.Berg \\
\hline Myrtaceae & & Myrciaria delicatula (DC.) O.Berg \\
\hline Myrtaceae & & Myrciaria floribunda (H.West ex Willd.) O.Berg \\
\hline Myrtaceae & & Myrciaria tenella (DC.) O.Berg \\
\hline Myrtaceae & & Pimenta pseudocaryophyllus (Gomes) Landrum \\
\hline Myrtaceae & & Psidium acutangulum DC. \\
\hline Myrtaceae & & Psidium cattleianum Sabine \\
\hline Myrtaceae & & Psidium giganteum Mattos \\
\hline Myrtaceae & & Psidium guajava L. \\
\hline Myrtaceae & & Psidium guineense Sw. \\
\hline Myrtaceae & & Psidium hians Mart. ex DC. \\
\hline Myrtaceae & & Psidium longipetiolatum D.Legrand \\
\hline Myrtaceae & & Psidium myrsinites DC. \\
\hline Myrtaceae & 2 & Psidium myrtoides O.Berg \\
\hline Myrtaceae & & Psidium nutans O.Berg \\
\hline Myrtaceae & & Psidium rufum Mart. ex DC. \\
\hline Myrtaceae & & Psidium sartorianum (O.Berg) Nied. \\
\hline Myrtaceae & & Siphoneugena crassifolia (DC.) Proença \& Sobral \\
\hline Myrtaceae & & Siphoneugena densiflora O.Berg \\
\hline Myrtaceae & & Siphoneugena guilfoyleiana Proença \\
\hline Nyctaginaceae & & Guapira areolata (Heimerl) Lundell \\
\hline Nyctaginaceae & 1 & Guapira graciliflora (Mart. ex Schmidt) Lundell \\
\hline Nyctaginaceae & 1,2 & Guapira noxia (Netto) Lundell \\
\hline Nyctaginaceae & & Guapira opposita (Vell.) Reitz \\
\hline Nyctaginaceae & & Guapira tomentosa (Casar.) Lundell \\
\hline Nyctaginaceae & & Neea macrophylla Poepp. \& Endl. \\
\hline Nyctaginaceae & 1,2 & Neea theifera Oerst. \\
\hline Nyctaginaceae & & Pisonia ambigua Heimerl \\
\hline Ochnaceae & & Ouratea acuminata (A.DC.) Engl. \\
\hline Ochnaceae & & Ouratea castaneifolia (DC.) Engl. \\
\hline Ochnaceae & & Ouratea ferruginea Engl. \\
\hline Ochnaceae & & Ouratea glaucescens (A.St.-Hil.) Engl. \\
\hline Ochnaceae & 1,2 & Ouratea hexasperma (A.St.-Hil.) Baill. \\
\hline Ochnaceae & & Ouratea ovalis (Pohl) Engl. \\
\hline Ochnaceae & & Ouratea parviflora (A.DC.) Baill. \\
\hline Ochnaceae & & Ouratea salicifolia (A.St.-Hil. \& Tul.) Engl. \\
\hline Ochnaceae & & Ouratea schomburgkii (Planch.) Engl. \\
\hline Ochnaceae & & Ouratea sellowii (Planch.) Engl. \\
\hline Ochnaceae & & Ouratea semiserrata (Mart. \& Nees) Engl. \\
\hline Ochnaceae & 1,2 & Ouratea spectabilis (Mart.) Engl. \\
\hline Olacaceae & & Heisteria citrifolia Engl. \\
\hline
\end{tabular}

\begin{tabular}{|c|c|c|}
\hline 10 & $1.6 \%$ & ARV \\
\hline 55 & $8.8 \%$ & $\mathrm{ARB} / \mathrm{ARV}$ \\
\hline 4 & $0.6 \%$ & $\mathrm{ARB} / \mathrm{ARV}$ \\
\hline 3 & $0.5 \%$ & ARV \\
\hline 4 & $0.6 \%$ & $\mathrm{ARB} / \mathrm{ARV}$ \\
\hline 7 & $1.1 \%$ & $\mathrm{ARV}$ \\
\hline 1 & $0.2 \%$ & ARV \\
\hline 38 & $6.1 \%$ & $\mathrm{ARB} / \mathrm{ARV}$ \\
\hline 208 & $33.3 \%$ & ARV \\
\hline 1 & $0.2 \%$ & ARV \\
\hline 133 & $21.3 \%$ & ARV \\
\hline 43 & $6.9 \%$ & ARV \\
\hline 17 & $2.7 \%$ & ARV \\
\hline 7 & $1.1 \%$ & ARV \\
\hline 1 & $0.2 \%$ & ARV \\
\hline 2 & $0.3 \%$ & ARV \\
\hline 2 & $0.3 \%$ & ARV \\
\hline 10 & $1.6 \%$ & ARV \\
\hline 1 & $0.2 \%$ & ARV \\
\hline 2 & $0.3 \%$ & ARV \\
\hline 1 & $0.2 \%$ & $\mathrm{ARB} / \mathrm{ARV}$ \\
\hline 4 & $0.6 \%$ & ARV \\
\hline 1 & $0.2 \%$ & ARV \\
\hline 1 & $0.2 \%$ & ARV \\
\hline 33 & $5.3 \%$ & $\mathrm{ARB} / \mathrm{ARV}$ \\
\hline 1 & $0.2 \%$ & ARV \\
\hline 1 & $0.2 \%$ & ARV \\
\hline 59 & $9.4 \%$ & ARV \\
\hline 110 & $17.6 \%$ & ARV \\
\hline 1 & $0.2 \%$ & $\mathrm{ARB} / \mathrm{ARV}$ \\
\hline 7 & $1.1 \%$ & ARV \\
\hline 1 & $0.2 \%$ & ARV \\
\hline 3 & $0.5 \%$ & ARV \\
\hline 16 & $2.6 \%$ & ARV \\
\hline 2 & $0.3 \%$ & ARV \\
\hline 1 & $0.2 \%$ & ARV \\
\hline 126 & $20.2 \%$ & ARV \\
\hline 225 & $36.0 \%$ & ARV \\
\hline 22 & $3.5 \%$ & $\mathrm{ARB} / \mathrm{ARV}$ \\
\hline 1 & $0.2 \%$ & $\mathrm{ARB} / \mathrm{ARV}$ \\
\hline 1 & $0.2 \%$ & $\mathrm{ARB} / \mathrm{ARV}$ \\
\hline 204 & $32.6 \%$ & ARV \\
\hline 1 & $0.2 \%$ & ARV \\
\hline 4 & $0.6 \%$ & ARV \\
\hline 98 & $15.7 \%$ & ARV \\
\hline 1 & $0.2 \%$ & ARV \\
\hline 5 & $0.8 \%$ & ARV \\
\hline 310 & $49.6 \%$ & ARV \\
\hline 2 & $0.3 \%$ & ARV \\
\hline 2 & $0.3 \%$ & $\mathrm{ARB} / \mathrm{ARV}$ \\
\hline 1 & $0.2 \%$ & ARV \\
\hline 1 & $0.2 \%$ & $\mathrm{ARB} / \mathrm{ARV}$ \\
\hline 3 & $0.5 \%$ & ARV \\
\hline 6 & $1.0 \%$ & $\mathrm{ARB} / \mathrm{ARV}$ \\
\hline 178 & $28.5 \%$ & ARV \\
\hline 12 & $1.9 \%$ & $\mathrm{ARB} / \mathrm{ARV}$ \\
\hline
\end{tabular}




\begin{tabular}{|c|c|c|}
\hline Olacaceae & & Heisteria densifrons Engl. \\
\hline Olacaceae & & Heisteria ovata Benth. \\
\hline Olacaceae & & Ximenia americana L. \\
\hline Oleaceae & & Priogymnanthus hasslerianus (Chodat) P.S.Green \\
\hline Opiliaceae & 1,2 & Agonandra brasiliensis Miers ex Benth. \& Hook.f. \\
\hline Opiliaceae & & Agonandra silvatica Ducke \\
\hline Pentaphylacaceae & & Ternstroemia brasiliensis Cambess. \\
\hline Peraceae & & Chaetocarpus echinocarpus (Baill.) Ducke \\
\hline Peraceae & & Pera glabrata (Schott) Poepp. ex Baill. \\
\hline Phyllanthaceae & & Richeria grandis Vahl \\
\hline Phyllanthaceae & & Savia dictyocarpa Müll.Arg. \\
\hline Phytolaccaceae & & Gallesia integrifolia (Spreng.) Harms \\
\hline Piperaceae & & Piper aduncum L. \\
\hline Polygonaceae & & Coccoloba densifrons Mart. ex Meisn. \\
\hline Polygonaceae & & Coccoloba glaziovii Lindau \\
\hline Polygonaceae & & Coccoloba latifolia Lam. \\
\hline Polygonaceae & & Coccoloba mollis Casar. \\
\hline Polygonaceae & & Coccoloba ramosissima Wedd. \\
\hline Primulaceae & & Cybianthus goyazensis Mez \\
\hline Primulaceae & & Myrsine coriacea (Sw.) R.Br. ex Roem. \& Schult. \\
\hline Primulaceae & 1,2 & Myrsine guianensis (Aubl.) Kuntze \\
\hline Primulaceae & & Myrsine leuconeura Mart. \\
\hline Primulaceae & & Myrsine umbellata Mart. \\
\hline Proteaceae & & Euplassa inaequalis (Pohl) Engl. \\
\hline Proteaceae & 1,2 & Roupala montana Aubl. \\
\hline Proteaceae & & Roupala rhombifolia Mart. ex Meisn. \\
\hline Rhamnaceae & 2 & Rhamnidium elaeocarpum Reissek \\
\hline Rhamnaceae & & Rhamnus sphaerosperma Sw. \\
\hline Rhamnaceae & & Ziziphus joazeiro Mart. \\
\hline Rosaceae & & Prunus myrtifolia (L.) Urb. \\
\hline Rubiaceae & 1,2 & Alibertia edulis (Rich.) A.Rich. \\
\hline Rubiaceae & & Alseis pickelii Pilg. \& Schmale \\
\hline Rubiaceae & & Amaioua guianensis Aubl. \\
\hline Rubiaceae & & Calycophyllum multiflorum Griseb. \\
\hline Rubiaceae & & Chomelia obtusa Cham. \& Schltdl. \\
\hline Rubiaceae & & Chomelia parviflora (Müll.Arg.) Müll.Arg. \\
\hline Rubiaceae & & Chomelia pohliana Müll.Arg. \\
\hline Rubiaceae & & Chomelia ribesioides Benth. ex A.Gray \\
\hline Rubiaceae & & Cordiera macrophylla (K.Schum.) Kuntze \\
\hline Rubiaceae & & Coussarea congestiflora Müll.Arg. \\
\hline Rubiaceae & & Coussarea contracta (Walp.) Müll.Arg. \\
\hline Rubiaceae & & Coussarea hydrangeifolia (Benth.) Müll.Arg. \\
\hline Rubiaceae & & Coutarea hexandra (Jacq.) K.Schum. \\
\hline Rubiaceae & & Duroia duckei Huber \\
\hline Rubiaceae & & Faramea bracteata Benth. \\
\hline Rubiaceae & & Faramea crassifolia Benth. \\
\hline Rubiaceae & & Faramea hyacinthina Mart. \\
\hline Rubiaceae & & Ferdinandusa elliptica (Pohl) Pohl \\
\hline Rubiaceae & & Ferdinandusa speciosa Pohl \\
\hline Rubiaceae & & Genipa americana L. \\
\hline Rubiaceae & & Guettarda angelica Mart. ex Müll.Arg. \\
\hline Rubiaceae & 1,2 & Guettarda viburnoides Cham. \& Schltdl. \\
\hline Rubiaceae & & Ixora brevifolia Benth. \\
\hline Rubiaceae & & Ladenbergia chapadensis S.Moore \\
\hline Rubiaceae & & Pagamea guianensis Aubl. \\
\hline Rubiaceae & & Palicourea coriacea (Cham.) K.Schum. \\
\hline
\end{tabular}

\begin{tabular}{|c|c|c|}
\hline 1 & $0.2 \%$ & LIA/ARB/ARV \\
\hline 69 & $11.0 \%$ & ARB/ARV \\
\hline 24 & $3.8 \%$ & ARB/ARV \\
\hline 21 & $3.4 \%$ & ARV \\
\hline 247 & $39.5 \%$ & $\mathrm{ARB} / \mathrm{ARV}$ \\
\hline 2 & $0.3 \%$ & ARV \\
\hline 1 & $0.2 \%$ & ARB/ARV \\
\hline 13 & $2.1 \%$ & ARB/ARV \\
\hline 96 & $15.4 \%$ & $\mathrm{ARB} / \mathrm{ARV}$ \\
\hline 2 & $0.3 \%$ & ARV \\
\hline 3 & $0.5 \%$ & ARV \\
\hline 1 & $0.2 \%$ & ARV \\
\hline 5 & $0.8 \%$ & $\mathrm{ARB} / \mathrm{ARV}$ \\
\hline 1 & $0.2 \%$ & LIA/ARB/ARV \\
\hline 2 & $0.3 \%$ & $\mathrm{ARB} / \mathrm{ARV}$ \\
\hline 1 & $0.2 \%$ & ARV \\
\hline 58 & $9.3 \%$ & ARV \\
\hline 1 & $0.2 \%$ & ARB/ARV \\
\hline 2 & $0.3 \%$ & ARB/ARV \\
\hline 29 & $4.6 \%$ & ARB/ARV \\
\hline 145 & $23.2 \%$ & ARB/ARV \\
\hline 1 & $0.2 \%$ & ARV \\
\hline 45 & $7.2 \%$ & ARV \\
\hline 43 & $6.9 \%$ & ARV \\
\hline 389 & $62.2 \%$ & ARB/ARV \\
\hline 1 & $0.2 \%$ & ARV \\
\hline 89 & $14.2 \%$ & ARV \\
\hline 8 & $1.3 \%$ & ARB/ARV \\
\hline 1 & $0.2 \%$ & ARV \\
\hline 17 & $2.7 \%$ & ARV \\
\hline 173 & $27.7 \%$ & $\mathrm{ARB} / \mathrm{ARV}$ \\
\hline 1 & $0.2 \%$ & ARV \\
\hline 32 & $5.1 \%$ & ARB/ARV \\
\hline 4 & $0.6 \%$ & ARV \\
\hline 17 & $2.7 \%$ & ARB/ARV \\
\hline 2 & $0.3 \%$ & ARB/ARV \\
\hline 14 & $2.2 \%$ & ARB/ARV \\
\hline 36 & $5.8 \%$ & ARB/ARV \\
\hline 53 & $8.5 \%$ & ARB/ARV \\
\hline 1 & $0.2 \%$ & ARB/ARV \\
\hline 1 & $0.2 \%$ & ARB/ARV \\
\hline 91 & $14.6 \%$ & ARB/ARV \\
\hline 3 & $0.5 \%$ & ARB/ARV \\
\hline 3 & $0.5 \%$ & ARV \\
\hline 1 & $0.2 \%$ & ARB/ARV \\
\hline 9 & $1.4 \%$ & ARB/ARV \\
\hline 2 & $0.3 \%$ & ARB/ARV \\
\hline 107 & $17.1 \%$ & ARV \\
\hline 1 & $0.2 \%$ & ARB/ARV \\
\hline 25 & $4.0 \%$ & ARV \\
\hline 2 & $0.3 \%$ & ARB/ARV \\
\hline 147 & $23.5 \%$ & ARB/ARV \\
\hline 1 & $0.2 \%$ & ARB/ARV \\
\hline 3 & $0.5 \%$ & ARV \\
\hline 2 & $0.3 \%$ & ARB/ARV \\
\hline 6 & $1.0 \%$ & SUB/ARB/ARV \\
\hline
\end{tabular}


Rubiaceae

Rubiaceae

Rubiaceae

Rubiaceae

Rubiaceae

Rubiaceae

Rubiaceae

Rubiaceae

Rubiaceae

Rubiaceae

Rubiaceae

Rutaceae

Rutaceae

Rutaceae

Rutaceae

Rutaceae

Rutaceae

Rutaceae

Rutaceae

Rutaceae

Rutaceae

Rutaceae

Rutaceae

Rutaceae

Salicaceae

Salicaceae

Salicaceae

Salicaceae

Salicaceae

Salicaceae

Salicaceae

Salicaceae

Salicaceae

Salicaceae

Salicaceae

Salicaceae

Sapindaceae

Sapindaceae

Sapindaceae

Sapindaceae

Sapindaceae

Sapindaceae

Sapindaceae

Sapindaceae

Sapindaceae

Sapindaceae

Sapindaceae

Sapindaceae

Sapindaceae

Sapindaceae

Sapindaceae

Sapindaceae

Sapindaceae

Sapindaceae

Sapindaceae

Sapindaceae
1,2 Palicourea rigida Kunth

Posoqueria latifolia (Rudge) Schult.

Psychotria carthagenensis Jacq.

Psychotria vellosiana Benth.

Randia armata (Sw.) DC.

Rudgea amazonica Müll. Arg.

1,2 Rudgea viburnoides (Cham.) Benth.

Simira hexandra (S.Moore) Steyerm.

Tocoyena brasiliensis Mart.

1,2 Tocoyena formosa (Cham. \& Schltdl.) K.Schum.

Tocoyena sellowiana (Cham. \& Schltdl.) K.Schum.

Dictyoloma vandellianum A.Juss.

Esenbeckia febrifuga (A.St.-Hil.) A. Juss. ex Mart.

Esenbeckia grandiflora Mart.

Esenbeckia pumila Pohl

Galipea jasminiflora (A.St.-Hil.) Engl.

Helietta apiculata Benth.

Hortia brasiliana Vand. ex DC.

Zanthoxylum gardneri Engl.

Zanthoxylum monogynum A.St.-Hil.

Zanthoxylum petiolare A.St.-Hil. \& Tul.

2 Zanthoxylum rhoifolium Lam.

Zanthoxylum riedelianum Engl.

Zanthoxylum rigidum Humb. \& Bonpl. exWilld.

Casearia aculeata Jacq.

Casearia arborea (Rich.) Urb.

Casearia decandra Jacq.

Casearia gossypiosperma Briq.

Casearia grandiflora Cambess.

Casearia javitensis Kunth

Casearia lasiophylla Eichler

Casearia rupestris Eichler

1,2 Casearia sylvestris Sw.

Casearia ulmifolia Vahl ex Vent.

Prockia crucis P.Browne ex L.

Xylosma ciliatifolia (Clos) Eichler

Allophylus edulis (A.St.-Hil. et al.) Hieron. ex Niederl.

Allophylus quercifolius (Mart.) Radlk.

Allophylus racemosus Sw.

Allophylus semidentatus (Miq.) Radlk.

Cupania diphylla Vahl

Cupania oblongifolia Mart.

Cupania paniculata Cambess.

Cupania polyodonta Radlk.

Cupania racemosa (Vell.) Radlk.

Cupania vernalis Cambess.

Diatenopteryx sorbifolia Radlk.

2 Dilodendron bipinnatum Radlk.

1,2 Magonia pubescens A.St.-Hil.

Matayba elaeagnoides Radlk.

1 Matayba guianensis Aubl.

Matayba inelegans Spruce ex Radlk.

Sapindus saponaria L.

Talisia angustifolia Radlk.

Talisia esculenta (Cambess.) Radlk.

Toulicia tomentosa Radlk.
$248 \quad 39.7 \% \quad$ SUB/ARB/ARV

$10.2 \% \quad \mathrm{ARV}$

$3 \quad 0.5 \% \quad \mathrm{ARB} / \mathrm{ARV}$

$6 \quad 1.0 \% \quad$ ARB/ARV

$10 \quad 1.6 \%$ LIA/ARB/ARV

$1 \quad 0.2 \% \quad \mathrm{ARB} / \mathrm{ARV}$

$13721.9 \% \quad \mathrm{ARB} / \mathrm{ARV}$

$1 \quad 0.2 \% \quad$ ARV

$12 \quad 1.9 \% \quad \mathrm{ARB} / \mathrm{ARV}$

$377 \quad 60.3 \%$ SUB/ARB/ARV

$1 \quad 0.2 \% \quad \mathrm{ARB} / \mathrm{ARV}$

$121.9 \%$ ARV

$3 \quad 0.5 \%$ ARV

$2 \quad 0.3 \% \quad \mathrm{SUB} / \mathrm{ARB} / \mathrm{ARV}$

$10.2 \% \quad \mathrm{ARB} / \mathrm{ARV}$

$10.2 \%$ ARV

$10.2 \%$ ARV

$4 \quad 0.6 \%$ ARB/ARV

$10.2 \%$ ARV

$10.2 \%$ ARV

$1 \quad 0.2 \%$ ARV

$90 \quad 14.4 \%$ ARV

$68 \quad 10.9 \%$ ARV

$11 \quad 1.8 \% \quad \mathrm{ARB} / \mathrm{ARV}$

$1 \quad 0.2 \% \quad \mathrm{ARB} / \mathrm{ARV}$

$29 \quad 4.6 \% \quad \mathrm{ARB} / \mathrm{ARV}$

$152.4 \%$ ARB/ARV

$9 \quad 1.4 \%$ ARV

$51 \quad 8.2 \% \quad \mathrm{ARB} / \mathrm{ARV}$

$6 \quad 1.0 \% \quad \mathrm{ARB} / \mathrm{ARV}$

$9 \quad 1.4 \% \quad \mathrm{ARB} / \mathrm{ARV}$

$152.4 \%$ ARV

$367 \quad 58.7 \%$ ARV

$30.5 \% \quad \mathrm{ARB} / \mathrm{ARV}$

$1 \quad 0.2 \% \quad \mathrm{ARB} / \mathrm{ARV}$

$2 \quad 0.3 \% \quad \mathrm{ARB} / \mathrm{ARV}$

$4 \quad 0.6 \% \quad$ ARB/ARV

$2 \quad 0.3 \% \quad \mathrm{ARB} / \mathrm{ARV}$

$10.2 \% \quad \mathrm{ARB} / \mathrm{ARV}$

$10.2 \%$ ARV

$10.2 \%$ ARV

$10.2 \%$ ARV

$10.2 \% \quad \mathrm{ARB} / \mathrm{ARV}$

$10.2 \%$ ARV

$20.3 \%$ ARV

$223.5 \%$ ARV

$1 \quad 0.2 \%$ ARV

104 16.6\% ARV

$26943.0 \%$ ARV

$12 \quad 1.9 \% \quad$ ARB/ARV

$142 \quad 22.7 \%$ ARB/ARV

$10.2 \%$ ARV

$10.2 \%$ ARV

$4 \quad 0.6 \%$ ARB/ARV

$5 \quad 0.8 \%$ ARV

$5 \quad 0.8 \%$ ARV 
Sapotaceae

Sapotaceae

Sapotaceae

Sapotaceae

Sapotaceae

Sapotaceae

Sapotaceae

Sapotaceae

Sapotaceae

Sapotaceae

Sapotaceae

Sapotaceae

Sapotaceae

Sapotaceae

Sapotaceae

Sapotaceae

Simaroubaceae

Simaroubaceae

Simaroubaceae

Simaroubaceae

Simaroubaceae

Siparunaceae

Siparunaceae

Solanaceae

Solanaceae

Solanaceae

Solanaceae

Solanaceae

Solanaceae

Solanaceae

Styracaceae

Styracaceae

Styracaceae

Styracaceae

Styracaceae

Styracaceae

Symplocaceae

Symplocaceae

Symplocaceae

Symplocaceae

Symplocaceae

Symplocaceae

Symplocaceae

Symplocaceae

Symplocaceae

Symplocaceae

Symplocaceae

Thymelaeaceae

Thymelaeaceae

Thymelaeaceae

Urticaceae

Urticaceae

Urticaceae

Urticaceae

Urticaceae

Verbenaceae
Chrysophyllum arenarium Allemão

Chrysophyllum gonocarpum (Mart. \& Eichler ex Miq.) Engl.

Chrysophyllum marginatum (Hook. \& Arn.) Radlk.

Chrysophyllum rufum Mart.

Ecclinusa ramiflora Mart.

Manilkara triflora (Allemão) Monach.

Micropholis egensis (A.DC.) Pierre

Micropholis gardneriana (A.DC.) Pierre

Micropholis venulosa (Mart. \& Eichler) Pierre

Pouteria caimito (Ruiz \& Pav.) Radlk.

Pouteria gardneri (Mart. \& Miq.) Baehni

Pouteria macrophylla (Lam.) Eyma

1,2 Pouteria ramiflora (Mart.) Radlk.

Pouteria reticulata (Engl.) Eyma

1,2 Pouteria torta (Mart.) Radlk.

Pouteria venosa (Mart.) Baehni

Simaba cedron Planch.

Simaba ferruginea A.St.-Hil.

Simaba floribunda A.St.-Hil.

Simarouba amara Aubl.

1,2 Simarouba versicolor A.St.-Hil.

Siparuna brasiliensis (Spreng.) A.DC.

1,2 Siparuna guianensis Aubl.

Solanum crinitum Lam.

Solanum grandiflorum Ruiz \& Pav.

Solanum granulosoleprosum Dunal

Solanum lacerdae Dusén

Solanum lycocarpum A.St.-Hil.

Solanum stipulaceum Willd. ex Roem. \& Schult.

Solanum variabile Mart.

Styrax camporum Pohl

1,2 Styrax ferrugineus Nees \& Mart.

Styrax leprosus Hook. \& Arn.

Styrax pallidus A.DC.

Styrax pedicellatus (Perkins) B.Walln.

Styrax pohlii A.DC.

Symplocos celastrinea Mart.

Symplocos guianensis (Aubl.) Gürke

Symplocos nitens (Pohl) Benth.

Symplocos oblongifolia Casar.

Symplocos pentandra (Mattos) Occhioni ex Aranha

Symplocos platyphylla (Pohl) Benth.

Symplocos pubescens Klotzsch exBenth.

Symplocos revoluta Casar.

Symplocos rhamnifolia A.DC.

Symplocos tenuifolia Brand

Symplocos uniflora (Pohl) Benth.

Daphnopsis fasciculata (Meisn.) Nevling

Daphnopsis racemosa Griseb.

Daphnopsis utilis Warm.

Cecropia glaziovii Snethl.

Cecropia hololeuca Miq.

Cecropia pachystachya Trécul

Cecropia palmata Willd.

Cecropia saxatilis Snethl.

Aloysia virgata (Ruiz \& Pav.) Juss. $\begin{array}{rrl}5 & 0.8 \% & \text { ARV } \\ 3 & 0.5 \% & \text { ARV } \\ 25 & 4.0 \% & \text { ARB/ARV } \\ 1 & 0.2 \% & \text { ARB/ARV } \\ 1 & 0.2 \% & \text { ARV } \\ 8 & 1.3 \% & \text { ARB/ARV } \\ 1 & 0.2 \% & \text { ARV } \\ 2 & 0.3 \% & \text { ARB/ARV } \\ 5 & 0.8 \% & \text { ARB/ARV } \\ 1 & 0.2 \% & \text { ARB/ARV } \\ 8 & 1.3 \% & \text { ARV } \\ 1 & 0.2 \% & \text { ARV } \\ 386 & 61.8 \% & \text { ARV } \\ 2 & 0.3 \% & \text { ARB/ARV } \\ 161 & 25.8 \% & \text { ARV } \\ 1 & 0.2 \% & \text { ARB/ARV } \\ 1 & 0.2 \% & \text { ARV } \\ 11 & 1.8 \% & \text { ARV } \\ 1 & 0.2 \% & \text { ARB/ARV } \\ 22 & 3.5 \% & \text { ARV } \\ 267 & 42.7 \% & \text { ARV }\end{array}$

$6 \quad 1.0 \% \quad \mathrm{ARB} / \mathrm{ARV}$

$12720.3 \% \quad \mathrm{ARB} / \mathrm{ARV}$

$9 \quad 1.4 \% \quad \mathrm{ARB} / \mathrm{ARV}$

$30.5 \% \quad$ ARB/ARV

$5 \quad 0.8 \% \quad$ ARV

$5 \quad 0.8 \% \quad \mathrm{ARB} / \mathrm{ARV}$

$106 \quad 17.0 \%$ ARB/ARV

$1 \quad 0.2 \% \quad \mathrm{ARB} / \mathrm{ARV}$

$1 \quad 0.2 \% \quad \mathrm{ARB} / \mathrm{ARV}$

$93 \quad 14.9 \% \quad \mathrm{ARB} / \mathrm{ARV}$

$186 \quad 29.8 \%$ ARB/ARV

$1 \quad 0.2 \% \quad$ ARV

$1 \quad 0.2 \% \quad \mathrm{ARB} / \mathrm{ARV}$

$10.2 \% \quad \mathrm{ARB} / \mathrm{ARV}$

$20.3 \% \quad \mathrm{ARB} / \mathrm{ARV}$

$4 \quad 0.6 \% \quad$ ARB/ARV

$1 \quad 0.2 \% \quad \mathrm{ARB} / \mathrm{ARV}$

$24 \quad 3.8 \% \quad$ ARB/ARV

$8 \quad 1.3 \%$ SUB/ARB/ARV

$1 \quad 0.2 \% \quad \mathrm{ARB} / \mathrm{ARV}$

$4 \quad 0.6 \% \quad \mathrm{ARB} / \mathrm{ARV}$

$193.0 \%$ ARB/ARV

$20.3 \% \quad$ ARV

$182.9 \% \quad$ ARB/ARV

$50.8 \% \quad \mathrm{ARB} / \mathrm{ARV}$

$5 \quad 0.8 \% \quad \mathrm{ARB} / \mathrm{ARV}$

$193.0 \%$ ARB/ARV

$20.3 \% \quad$ ARB/ARV

$30.5 \% \quad \mathrm{ARB} / \mathrm{ARV}$

$10.2 \%$ ARV

$20.3 \%$ ARV

$77 \quad 12.3 \%$ ARV

$1 \quad 0.2 \%$ ARV

$20.3 \%$ ARV

$6 \quad 1.0 \% \quad \mathrm{ARB} / \mathrm{ARV}$ 
Verbenaceae

Vochysiaceae

Vochysiaceae

Vochysiaceae

Vochysiaceae

Vochysiaceae

Vochysiaceae

Vochysiaceae

Vochysiaceae

Vochysiaceae

Vochysiaceae

Vochysiaceae

Vochysiaceae

Vochysiaceae

Vochysiaceae

Vochysiaceae

Vochysiaceae

Vochysiaceae

Vochysiaceae

Vochysiaceae

Vochysiaceae

Vochysiaceae

Vochysiaceae

Vochysiaceae

Vochysiaceae

Vochysiaceae

Vochysiaceae

Vochysiaceae
Lantana camara L.

1,2 Callisthene fasciculata Mart.

Callisthene hassleri Briq.

Callisthene major Mart. \& Zucc.

Callisthene microphylla Warm.

Callisthene minor Mart.

Callisthene molissima Warm.

Qualea cordata (Mart.) Spreng.

Qualea cryptantha (Spreng.) Warm.

Qualea dichotoma (Mart.) Warm.

1,2 Qualea grandiflora Mart.

1,2 Qualea multiflora Mart.

1,2 Qualea parviflora Mart.

1,2 Salvertia convallariodora A.St.-Hil.

Vochysia cinnamomea Pohl

Vochysia divergens Pohl

Vochysia elliptica Mart.

2 Vochysia gardneri Warm.

2 Vochysia haenkeana Mart.

Vochysia magnifica Warm.

Vochysia petraea Warm.

Vochysia pyramidalis Mart.

1,2 Vochysia rufa Mart.

Vochysia spathiphylla Stafleu

Vochysia thyrsoidea Pohl

Vochysia tomentosa (G.F.W.Meyer) DC.

Vochysia tucanorum Mart.

Vochysia vismiifolia Spruce ex Warm.

$\begin{array}{rrl}12 & 1.9 \% & \text { SUB/ARB/ARV } \\ 127 & 20.3 \% & \text { ARV } \\ 21 & 3.4 \% & \text { ARB/ARV } \\ 46 & 7.4 \% & \text { ARV } \\ 3 & 0.5 \% & \text { ARB/ARV } \\ 10 & 1.6 \% & \text { ARV } \\ 22 & 3.5 \% & \text { ARV } \\ 29 & 4.6 \% & \text { ARV } \\ 1 & 0.2 \% & \text { ARB/ARV } \\ 53 & 8.5 \% & \text { ARB/ARV } \\ 526 & 84.2 \% & \text { ARV } \\ 343 & 54.9 \% & \text { ARB/ARV } \\ 485 & 77.6 \% & \text { ARB/ARV } \\ 310 & 49.6 \% & \text { ARV } \\ 77 & 12.3 \% & \text { ARV } \\ 4 & 0.6 \% & \text { ARB/ARV } \\ 101 & 16.2 \% & \text { ARB/ARV } \\ 64 & 10.2 \% & \text { ARV } \\ 64 & 10.2 \% & \text { ARV } \\ 2 & 0.3 \% & \text { ARV } \\ 1 & 0.2 \% & \text { ARV } \\ 2 & 0.3 \% & \text { ARV } \\ 223 & 35.7 \% & \text { ARV } \\ 1 & 0.2 \% & \text { ARB/ARV } \\ 80 & 12.8 \% & \text { ARV } \\ 1 & 0.2 \% & \text { ARV } \\ 89 & 14.2 \% & \text { ARV } \\ 1 & 0.2 \% & \text { ARV } \\ & & \\ 5 & \end{array}$



CERRADO

O Cerrado, principal savana neotropical, possui elevada biodiversidade, e grande diversidade de paisagens. O elevado valor biológico do bioma é consequência de um isolamento geográfico de outras savanas, das condições climáticas atuais e pretéritas e da distribuição de diferentes formações vegetais em forma de mosaico, além da influência dos demais biomas da América do Sul. Determinar os padrões espaciais da biodiversidade é fundamental na biogeografia, especialmente para priorizar pesquisas biológicas e direcionar políticas de conservação. Algumas propostas de subdivisões foram realizadas no Cerrado, no entanto ainda há necessidade de agregar novas informações a essas propostas. Nossos objetivos foram delimitar os Distritos Biogeográficos (DB) do Cerrado com base na similaridade de comunidades de plantas arbóreas. Utilizamos uma base de dados ampliada e revisada, caracterizamos os DB em relação ao clima, espécies indicadoras e estado de conservação. Identificamos sete DB, dos quais os três das regiões extremas do Cerrado, apresentaram maiores dissimilaridades em relação aos demais. Esses DB apresentaram, como espécies indicadoras, menor número de espécies típicas do cerrado, tendo influência dos biomas adjacentes. Os maiores números de espécies típicas de cerrado nos DB Centro-oeste, que possui maior extensão, Centro, que corresponde às áreas altas do Cerrado, e Nordeste, que é a região de maior estabilidade climática desde as glaciações do Quaternário. Temperatura mínima anual, sazonalidade da temperatura e precipitação anual são as variáveis mais importantes para a diferenciação dos grupos. Os DB apresentaram dois principais padrões climáticos. Os DB Sul, DB Sudeste e DB Sudoeste possuem menores temperaturas mínimas e maior sazonalidade da temperatura, enquanto os DB Centro-oeste, DB Norte e DB Nordeste apresentaram padrão oposto. O DB Centro apresentou tanto baixas temperaturas mínimas, quanto baixa sazonalidade da temperatura. Em termos de conservação, os DB apresentam grande variação nos níveis de desmatamento e de áreas protegidas. Os DB, aliados a ferramentas e técnicas consolidadas, podem servir de ponto de partida para equilibrar os esforços de conservação do bioma Cerrado. 
A identificação de unidades biologicamente homogêneas é a mais antiga abordagem da

624

625

626

627

628

629

630

631

632

633

634

635

636

637

638

639

640

641

642

643

644

645

646

647

648

649

650

651 Biogeografia. Nas ciências naturais, essa ferramenta foi usada inicialmente para direcionar pesquisas biológicas, especialmente durante os séculos XIX e XX e, a partir disso, usada na chamada Biogeografia Aplicada (Diamond 1975, Wilson e Willis 1975, Ladle e Whitaker 2011). Diversas propostas em escala global ou continental foram apresentadas, tendo como base as formações vegetais, as comunidades de fauna, comunidades florísticas ou combinações dessas (Clements \& Shelford 1939; Dice 1943; Udvardy 1975; Wallace 1876).

Uma proposta unificando as terminologias e experiências anteriores (Udvardy 1975), resultou em uma divisão hierárquica composta por Reinos, Províncias e Distritos Biogeográficos (DB). Os reinos seguiram a proposta das regiões de fauna de Wallace (1876), e as Províncias Biogeográficas, abrangendo grandes regiões, seriam a subdivisão desses reinos com o uso de províncias bióticas (Dice 1943), as quais têm como critério a combinação de fatores como vegetação, clímax ecológico, flora, fauna, fisiografia e solos. O conceito de províncias bióticas é semelhante ao de bioma, definido como um complexo de organismos englobando a vegetação clímax e suas subdivisões, além de componentes da fauna, ocupando extensas regiões sob um mesmo domínio climático (Clements \& Shelford 1939; Oxford 2010). Os DB são baseados em diferenças mais sutis dentro das Províncias Biogeográficas. Esses distritos são fundamentais para subsidiar iniciativas de conservação da biodiversidade (Udvardy 1975).

A partir de 1829, surgiram diversas propostas de classificação da vegetação brasileira, com pouca variação conceitual, porém com diferenças importantes entre os limites estabelecidos (e. g. Torello-Raventos et al. 2013; Smith 1961; Rizzini 1963b; Rizzini \& Pinto 1964; Veloso 1964; Veloso \& Strang 1970; Morrone 2001). Atualmente, a delimitação dos biomas brasileiros (IBGE 2004a), baseada na classificação da vegetação (IBGE 2004b), apresenta os biomas Amazônia, Cerrado, Caatinga, Mata Atlântica, Pampas e Pantanal.

O Cerrado é uma formação vegetal predominantemente savânica, composta por um mosaico de áreas abertas e fechadas (Eiten 1972; Ribeiro \& Walter 2008). Ocupa principalmente áreas altas, como o escudo central brasileiro, as Chapadas e serras, mas também ocorrem em vales e depressões periféricas das terras altas. Essa complexa paisagem, e sua localização geográfica são responsáveis pelas grandes diferenças climáticas observadas no 
652

653

654

655

656

657

658

659

660

661

662

663

664

665

666

667

668

669

670

671

672

673

674

675

676

677

678

679

680

681

682

Cerrado (Furley \& Ratter 1988). O clima é um importante preditor das comunidades vegetais, moldando diferentes ecossistemas sobre a superfície da Terra (Bongers et al. 1999; Castro 1994; Greve et al. 2011; Holmgren \& Poorter 2007; Kubota et al. 2014; Mckenzie et al. 2003). Algumas condições climáticas nas savanas, como umidade e sazonalidade, selecionam as comunidades regionais (Furley 2006; Lehmann et al. 2014).

O Cerrado, a Caatinga e o Chaco compõem os biomas abertos da América do Sul (Sarmiento 1975). As formações abertas são predominantes durante os períodos mais frios (glaciações), e têm sua distribuição reduzida nos períodos interglaciais, quando as formações florestais se expandem pelo continente (Haffer 1969). Nos dias atuais, a Amazônia e a Mata Atlântica, os principais biomas florestais da América do Sul, estão separados pelo Cerrado, mas elementos dos dois biomas são encontrados nas matas ciliares e matas de galeria do Cerrado (Méio et al. 2003; Oliveira-Filho \& Ratter 1995). Como consequência relictual das flutuações climáticas, diversos encraves com vegetação de Cerrado são encontrados nos biomas adjacentes (Vanzolini \& Williams 1981).

Esse constante fluxo da vegetação como consequência das flutuações climáticas (Cole 1960), pode ter proporcionado ampla troca de espécies entre os diferentes domínios fitogeográficos brasileiros (Salgado-Labouriau 2005), especialmente nas áreas de transição, onde a riqueza de plantas lenhosas é maior (Castro 1994). Essa situação tem como consequência a colonização bidirecional de diversas espécies ou gêneros entre o Cerrado e os biomas adjacentes (Caetano et al. 2008; Colli 2005; Novaes et al. 2010; Oliveira-Filho \& Ratter 1995; Ramos et al. 2009; Salgado-Labouriau 2005; Scariot \& Sevilha 2005; Simon et al. 2009). Tal situação se reflete na grande importância dos outros biomas para a flora do Cerrado (Castro et al. 1998; Heringer et al. 1977; Rizzini 1963b).

Apesar de sua diversidade, distribuição e relevância, o Cerrado já foi reduzido a cerca da metade do seu tamanho original (MMA 2006; Unesco 2000), em decorrência principalmente da expansão agropecuária (MMA 2002). Essa realidade vem se concretizando de maneira contínua e intensa, especialmente em algumas fitofisionomias (Bianchi \& Haig 2012). Estimativas apontam que, em poucas décadas, o Cerrado estará restrito somente às áreas protegidas, caso o ritmo de conversão se mantenha nos níveis atuais (Klink \& Machado 2005). A despeito de sua importância biológica, apenas 1,8\% são efetivamente protegidos por unidades de conservação de proteção integral. 
684

685

686

687

688

689

690

691

692

693

694

695

696

697

698

699

700

701

702

703

704

705

706

707

708

709

710

711

712

biotas regionais implicam na necessidade de caracterizar a biodiversidade regional, para que possam ser aplicadas medidas diferenciadas de conservação. Algumas propostas de subdivisões desse bioma foram realizadas, pautadas em atributos físicos (Arruda et al. 2008; Cochrane et al. 1985) ou florísticos (Castro 1994; Ratter \& Dargie 1992; Ratter et al. 1996, 2003).

Castro (1994) dividiu o Cerrado em oito grupos, com base em composição florística. Por sua vez, esses grupos se juntam formando três supercentros, determinados pelo polígono das secas e pelo polígono das geadas (Castro 1994). As regiões fisiogeográficas propostas por Cochrane et al. (1985), e revisitadas por Silva et al. (2006) são baseadas em aspectos físicos, como relevo, litologia e tipos de solos. Essa proposta foi corroborada pela maior diversidade beta entre essas regiões fisiográficas estudadas (Felfili et al. 2004). Combinando características físicas e oito taxa botânicos, Arruda et al. (2008) propuseram vinte e duas ecorregiões para o Cerrado. Onze Províncias Fitogeográficas baseadas na distribuição do gênero Mimosa foram sugeridas para o bioma Cerrado (Simon \& Proença 2000). Em uma série de estudos florísticos das espécies lenhosas, Ratter e colaboradores (Ratter \& Dargie 1992; Ratter et al. 1996, 2003, 2011) identificaram seis províncias florísticas na área núcleo do Cerrado, além de duas regiões disjuntas na Amazônia.

Caracterizar os padrões de distribuição das espécies em ecossistemas megadiversos é o primeiro passo para a compreensão dos mecanismos que geraram esses padrões. Em tempos de aceleradas mudanças climáticas, é importante entender como a biota responde às variações climáticas, para que se possam prever eminentes perdas biológicas (Pearson 2006) e elaborar propostas para minimizar tais perdas (Faleiro et al. 2013). Além disso, identificar as espécies responsáveis pela estruturação dos padrões biogeográficos e avaliar seu estado de conservação é de extrema importância para o conhecimento da biota regional. Apesar de numerosos, os estudos biogeográficos do Cerrado pouco se ativeram a essas questões.

Dessa forma, o presente estudo teve como objetivos identificar os Distritos Biogeográficos do Cerrado com uma base de dados atualizada e ampliada, e caracterizá-las quanto aos limites climáticos, espécies indicadoras e estado de conservação. Para isso foram testadas as seguintes hipóteses: (1) processos biogeográficos possibilitaram a formação de padrões espaciais na distribuição de plantas arbóreas do Cerrado e tais padrões são suficientes 
para a identificação de Distritos Biogeográficos; (2) esses distritos se diferenciam quanto ao clima e (3) são singulares quanto à associação das espécies que suportam tais regiões.

\section{MÉTODOS}

Área de estudo e base de dados

O estudo foi realizado no bioma Cerrado e utilizamos dados florísticos de espécies arbóreas de 448 inventários e levantamentos expeditos nos ambientes de interesse. No caso particular deste estudo, denominaremos como cerrado sentido amplo o conjunto de fitofisionomias formadas pelo cerrado sentido restrito e pelo o cerradão, devido à sua semelhança florística com o cerrado sentido restrito (Ribeiro \& Walter 2008). Dessa forma, todos os registros obtidos nos trabalhos pesquisados e nas bases de dados consultadas referem-se às espécies vegetais de porte arbóreo associadas com o cerrado sentido amplo por toda a extensão dos limites do Cerrado (IBGE 2004a) (Figura 1). Incluímos também algumas localidades do Piauí, pois em alguns pontos há a predominância de vegetação característica de cerrado, sendo contíguas ao limite do bioma. Nosso universo de análise é composto por 786 espécies distribuídas em 75 famílias. Revisamos a taxonomia e nomenclatura pelas atualizações do APG III no sítio eletrônico da Flora do Brasil (JBRJ 2014).

\section{Análises}

Para delimitar os Distritos Biogeográficos (DB) no Cerrado, realizamos uma análise de agrupamento baseada em uma matriz de similaridade entre as localidades. Para o cálculo da matriz de similaridade, excluímos as espécies que não contribuem para a formação de padrões. As espécies que excluímos foram aquelas consideradas raras, as de distribuição ocasional e as de ampla distribuição. Consideramos espécies raras aquelas presentes em menos de cinco localidades (1\%), e amplamente distribuídas as presentes em mais de 269 localidades (60\%). As espécies ocasionais são as de ocorrência predominante em outros biomas ou fitofisionomias, mas ocasionalmente registradas em estudos de cerrado.

As espécies não se distribuem ao acaso pela paisagem, sendo seu padrão natural de distribuição geográfica agrupado (Lieberman \& Lieberman 1994). Quando a área de distribuição das espécies foi maior ou igual ao observado ao acaso, sua distribuição foi considerada ocasional. Para identificar essas situações, realizamos aleatorizações de Monte Carlo da área de 
distribuição de cada espécie calculadas pelo mínimo polígono convexo, usando o pacote adehabitatHR (Calenge 2006) no aplicativo R (R Development Core Team 2013). Calculamos 1.000 polígonos mínimos convexos aleatórios para espécies que ocorressem entre cinco e 420 localidades. O intervalo de confiança foi estabelecido em 0,05. Padronizamos esses valores pela área de ocorrência das espécies.

A similaridade utilizada no agrupamento das comunidades foi calculada pelo índice de Sørensen no pacote recluster (Dapporto et al. 2013), usado para dados binários e recomendado por não considerar as ausências compartilhadas em sua equação (Meyer et al. 2004). Considerando que o índice de similaridade baseado em presenças compartilhadas tende a agrupar localidades com baixo número de espécies com as localidades que possuem grande número delas, removemos as localidades com baixa riqueza. Removemos qualquer localidade que tivesse uma riqueza inferior à média menos o desvio padrão do número final de espécies de todas as localidades.

Dentre sete métodos de agrupamento hierárquico aglomerativo (ward, single, complete, average, mcquitty, median e centroid), usamos o método average (Unweighted Pair Group Method with Arithmetic Mean - UPGMA) por apresentar a maior correlação entre a matriz de distância e a matriz cofenética (Sokal \& Rohlf 1962). Agrupamos a matriz de distância usando o pacote hclust (Rousseeuw et al. 2014). Estabelecemos três diferentes níveis de corte de similaridade para maximizar não somente o número de grupos, mas também o maior número de localidades em cada grupo. Desprezamos os grupos compostos por menos de dez localidades.

Para os grupos resultantes, realizamos uma análise de espécies indicadoras (Dufrêne \& Legendre 1997) com o objetivo de identificar o melhor agrupamento dentre os diferentes níveis de dissimilaridades. Usamos o mínimo valor médio do valor de $p$ das espécies indicadoras como valor limite para a definição dos grupos (McCune \& Grace 2002). Verificamos a significância do agrupamento por uma análise de variância da matriz de dissimilaridade explicada pelos grupos no pacote vegan (Oksanen et al. 2014). A estrutura espacial, gerada a partir das coordenadas espaciais com o pacote packfor (Dray et al. 2011), foi usada como co-variável para controlar o efeito do espaço. Delimitamos os DB pela congruência da distribuição das localidades, de modo que a maioria das comunidades pertencentes ao mesmo agrupamento permanecesse no 
mesmo DB. Quando possível, usamos acidentes do relevo, como rios de primeira ordem e divisores de relevo importantes, para delimitar as bordas dos DB.

Para o agrupamento selecionado, usamos a análise de espécies indicadoras para verificar qual a importância de cada bioma na formação dos grupos. Para isso, caracterizamos as espécies indicadoras quanto ao seu bioma de ocorrência (capítulo 1).

Para a caracterização climática das regiões biogeográficas usamos as variáveis Bio01 (temperatura média anual em 으), Bio04 (sazonalidade da temperatura, medida pelo desvio padrão em ำ), Bio05 (temperatura máxima anual em ํㅡ), Bio06 (temperatura mínima anual em 으), Bio12 (precipitação anual em mm), Bio14 (precipitação da semana mais seca em $\mathrm{mm}$ ), Bio15 (sazonalidade da precipitação medida pelo desvio padrão em mm), Bio20 (radiação média anual em $\mathrm{W} \mathrm{m}^{-2}$ ), Bio28 (índice médio de umidade anual) e Bio31 (sazonalidade do índice de umidade em desvio padrão). Essas variáveis climáticas são o resultado da interpolação da média dos dados climáticos mensais de estações climáticas no período de 30 (1960-1990) a 50 anos (1950-2000), de acordo com a sua disponibilidade (Hijmans et al. 2004).

Testamos a diferença entre as características ambientais das regiões usando uma análise multivariada de variância (Manova). Posteriormente, usando o pacote MASS (Venables \& Ripley 2002), realizamos uma análise discriminante por passos para verificar quais variáveis melhor diferenciam as regiões fitogeográficas. As variáveis que melhor predizem os grupos foram selecionadas pelo critério de Uschi que estima a confiabilidade da regra de classificação. Usando as variáveis selecionadas pela discriminantes por passos, testamos a diferença entre os DB com o teste Kruskall-Wallis com correção de Bonferroni. Posteriormente, usamos o teste Duun com o pacote dunn.test (Dinno 2014) para identificar entre quais pares de DB haviam diferenças significativas. Gráficos do tipo boxplot auxiliaram na interpretação da distribuição das variáveis climáticas. Fizemos essas análises no aplicativo R ( $\mathrm{R}$ Development Core Team 2013).

Quantificamos o desmatamento e a cobertura de unidades de conservação de proteção integral para cada região, usando a função tabulate area no ArcGis (ESRI 2011). Essas informações estão disponíveis em http://mapas.mma.gov.br/i3geo/datadownload.htm. 
801

802

803

804

805

806

807

808

809

810

811

812

813

814

815

816

817

818

819

820

821

822

823

824

825

Recuperamos três diferentes agrupamentos das localidades, de acordo com o nível de corte (Figura 2). No corte com um nível de dissimilaridade de 0,70 encontramos três regiões biogeográficas nas extremidades do Cerrado e uma grande região central. No segundo corte $(0,65)$ houve apenas mais uma divisão do grupo central, e no nível seguinte $(0,60)$ dois grupos emergiram desses dois grupos centrais (Figura 2). Selecionamos o terceiro agrupamento, no nível de dissimilaridade de 0,60, por apresentar o menor valor médio do valor de $p$ das análises de espécies indicadoras (Figura 2). Observamos que existem diferenças significativas entre a similaridade dos $D B\left(R_{6,150}=57,76 ; p=0,001\right)$ (Tabela 1$)$.

Os DB recuperadas apresentaram grande concordância com os padrões encontrados por Ratter et al. (2003) (Figura 3), porém com subdivisões na maioria delas. Dessa forma, sempre que possível mantivemos a mesma nomenclatura sugerida por esses autores. São eles: Distrito Biogeográfico Centro (DBC), Distrito Biogeográfico Centro-oeste (DBCW), Distrito Biogeográfico Norte (DBN), Distrito Biogeográfico Nordeste (DBNE), Distrito Biogeográfico Sul (DBS), Distrito Biogeográfico Sudeste (DBSE) e Distrito Biogeográfico Sudoeste (DBSW). Os dados das regiões DA (disjunct amazonian sites) e FWM (widely spread sites of strong mesotrophic character) encontradas por Ratter et al. (2003) não foram incluídos nessa análise, portanto esses DB não aparecem nesse agrupamento.

A análise de espécies indicadoras para o agrupamento selecionado apresentou 263 espécies indicadoras distribuídas entre os sete grupos, das quais 213 foram significativas (Material Suplementar 1). A maioria das espécies é indicadora do DBS, e os DBSW e DBNE apresentam os menores números. Poucas espécies de ampla distribuição suportam os grupos (Figura 4). Os maiores números de Espécies Predominantemente do Cerrado (EPC, como definido no cap. 1) dentre as espécies indicadoras estão nos DBC, DBCW e DBNE. O DBN foi o único com contribuição expressiva da Caatinga. Poucas espécies compartilhadas com a Amazônia sustentam esse agrupamento.

A importância das variáveis climáticas utilizadas para a caracterização dos DB variou conforme pode ser visto na Tabela 2 e Figura 5. As variáveis mais importantes para diferenciálos foram temperatura mínima anual, sazonalidade da temperatura e precipitação anual, com uma taxa de acerto da predição de 0,81 . Os DBN e DBS tiveram $100 \%$ de acerto de predição 
com esse modelo (Tabela 3; Figura 6) e as maiores taxas de erro foram entre DBNE e DBCW. 0 único par que não apresentou diferenças entre as variáveis climáticas selecionadas foram os DBS e DBSE (Tabela 4).

Os DB apresentaram dois principais padrões climáticos. Os DBS, DBSE e DBSW possuem menores temperaturas mínimas e maior sazonalidade da temperatura, enquanto os DBCW, DBN e DBNE apresentaram padrão oposto. A única exceção é o DBC, que apresentou tanto baixas temperaturas mínimas quanto baixa sazonalidade da temperatura. Há grande variação nos parâmetros climáticos do DBNE, especialmente em precipitação anual.

Observamos grande variação de cobertura vegetal remanescente e áreas protegidas entre os DB (Figura 7, Tabela 5). Os remanescentes naturais do DBS apresentaram os menores níveis de cobertura vegetal remanescentes (11\%), enquanto o DBNE apresentou o maior percentual (75\%). As áreas protegidas são também distribuídas de maneira desuniforme, ocupando $0,34 \%$ da área total do DBS e $6,18 \%$ no DBNE.

\section{Caracterização dos Distritos Biogeográficos do Cerrado}

Distrito Biogeográfico Sul (DBS). Corresponde ao Cerrado do estado de São Paulo, destacando-se na primeira divisão do cluster. A grande especificidade desse grupo é corroborada pela grande quantidade de espécies indicadoras, por marcada influência da Mata Atlântica e por evidente diferenciação climática, com invernos rigorosos e verões quentes. A área remanescente é de apenas $11 \%$ da sua cobertura original, sendo que menos de $0,35 \%$ de sua área total está legalmente protegida (Tabela 5).

Distrito Biogeográfico Sudoeste (DBSW). É composto por poucas localidades contíguas ao Pantanal. A maioria das espécies que suportam esse grupo é compartilhada com a Mata Atlântica, além de uma compartilhada com a Caatinga. Assim como o DBS, essa região apresenta invernos rigorosos e verões quentes. Esse grupo aparece na primeira divisão do cluster, evidenciando suas diferenças em relação às demais comunidades arbóreas de cerrado. Sua cobertura remanescente é de 35\%, estando apenas 1,15\% em áreas legalmente protegidas.

Distrito Biogeográfico Norte (DBN). Também bastante dissimilar das demais regiões, está localizado nas áreas baixas do Cerrado, incluindo parte da zona costeira do Maranhão e oeste do Piauí. Localizado em transição com a Caatinga, esse grupo é sustentado basicamente 
por espécies partilhadas com esse bioma. As temperaturas mínimas dessa região são as maiores observadas no Cerrado. Sua área remanescente é de $75 \%$, com menos de $3 \%$ de áreas legalmente protegidas.

Distrito Biogeográfico Nordeste (DBNE). Corresponde ao oeste baiano, extremo norte de Minas Gerais, sul do Maranhão, oeste do Piauí e porção leste do Tocantins (região norte da bacia do Rio Tocantins). Apesar da grande variabilidade climática observada nesse DB, ele é determinado por uma alta proporção de EPC. Nesse grupo são observadas grandes amplitudes das variáveis climáticas. Essa região possui maior área remanescente (75\%) e maior percentual de áreas legalmente protegidas (6,18\%).

Distrito Biogeográfico Centro-oeste $(D B C W)$. Cobre grande extensão do oeste do Cerrado, ocupando a bacia dos Rios Araguaia e Tocantins (especialmente porção sul), além de parte das bacias dos Rios Paraná e Paraguai. Esse DB possui grande extensão territorial, o que confere grande variação climática. A influência do domínio Amazônico sobre esse grupo é predominante. Cerca de metade das áreas naturais é remanescente (49\%), com menos de $2 \%$ protegidas.

Distrito Biogeográfico Sudeste (DBSE). Região de invernos rigorosos e verões quentes. Está localizado principalmente no estado de Minas Gerais, acompanhando os tributários da cabeceira do Rio São Francisco e Espinhaço mineiro. A maioria das espécies indicadoras desse grupo é compartilhada com a Mata Atlântica, porém o número de EPC não é desprezível. 0 total de remanescente é de $48 \%$, e a cobertura de áreas legalmente protegidas é de 2,57\%.

Distrito Biogeográfico Centro (DBC). Localizado nas áreas altas do planalto central, abrange o leste do estado de Goiás e oeste de Minas Gerais. É uma região climaticamente peculiar, pois apresenta baixas temperaturas mínimas e também baixa sazonalidade. É caracterizado principalmente por possuir espécies compartilhadas com a Mata Atlântica e por EPC. Pouco desse DB ainda permanece com cobertura nativa (33\%), e o percentual de áreas legalmente protegidas é inexpressivo (1,09\%).

\section{DISCUSSÃO}

A ampliação da base de dados e o refinamento metodológico nos proporcionaram a recuperação de três novos DB do Cerrado. A caracterização climática é um importante avanço 
para o entendimento dos determinantes das comunidades arbóreas do Cerrado. 0 conhecimento da distribuição das espécies que determinam as divisões do Cerrado levantam novas questões sobre a história dessa vegetação. Além disso, quantificamos os esforços de conservação e a perda de hábitat regional, o que pode direcionar novos planejamentos de conservação. Em geral, os padrões de distribuiç̧ão dos DB foram condizentes com algumas das propostas anteriores.

As regiões propostas por Arruda et al. (2008), por Cochrane et al. (1985) e Silva (2006) trazem regiões descontínuas e com pouco ou nenhum suporte biológico. As Unidades Ecológicas de Cochrane et. al (1985), identificadas com base em aspectos físicos, como solos e geomorfologia, foram estabelecidas para subsidiar atividades agrícolas, não se propondo a delimitar diferentes comunidades naturais. Silva et al. (2006) utilizaram classes de vegetação para a identificação de padrões gerais para a regionalização do Cerrado. Essa camada de informação reflete a estrutura da vegetação, mas não a composição florística das comunidades. Dessa forma, presume-se que os objetivos desses estudos foram identificar áreas putativamente semelhantes no bioma, mas não a delimitação de comunidades vegetais distintas.

As Províncias Fitogeográficas baseadas no gênero Mimosa (Simon \& Proença 2000) têm grande concordância com os DBCW (Províncias Fitogeográficas 2, 3 e 9), DBSE (Províncias Fitogeográficas 7 e 8) e DBC (Província Fitogeográfica 5), mostrando congruência entre os processos de dispersão das mimosas e das comunidades arbóreas do cerrado. As ecorregiões do Cerrado (Arruda et al. 2008) foram elaboradas pelo cruzamento de mapas físicos, climáticos e de vegetação, além das próprias unidades ecológicas de Cochrane et al. (2006). Nesse estudo foram também utilizados dados de ocorrência de oito taxa botânicos (três famílias e cinco gêneros). Duas das ecorregiões do Cerrado, (complexo Bodoquena e Paranapanema-Grande), são coincidentes com os DBSW e DBS. Em termos de endemismo, os mesmos padrões do presente estudo foram observados para a ecorregião Complexo Bodoquena (Arruda et al 2008) (DBSW), caracterizado por baixo endemismo, e muitas espécies de ampla distribuição, e baixo número de espécies endêmicas para a ecorregião Paranapanema-Grande.

O padrão por nós recuperado também foi encontrado por Ratter et al. (2003). No entanto, observamos três novos DB, o que representa um melhor refinamento da proposta anterior e um possível avanço no entendimento dos padrões biogeográficos das árvores do 
cerrado. Os DB ainda corroboraram os três supercentros do Cerrado (Castro 1994), correspondentes ao cerrado de São Paulo, do Planalto Central e do Nordeste. Esses supercentros são estruturados pelos polígonos da geada e da seca, o que coincide com as variáveis de precipitação anual e temperatura mínima, observadas para diferenciação dos DB. A diferenciação climática entre os DB demonstra a heterogeneidade dessa vegetação, o que nem sempre é observado em subdivisões regionais de comunidades vegetais (Gonçalves \& Souza 2014).

Os diferentes níveis de agrupamento nos permitiram identificar padrões hierárquicos nas comunidades vegetais do Cerrado. As regiões periféricas do bioma possuem características climáticas extremas, diferenciando suas comunidades da região nuclear do Cerrado. No norte, as altas temperaturas observadas ao longo do ano, determinam a influência da Caatinga. A transição entre os biomas Caatinga e Cerrado é caracterizada por uma paisagem extremamente diversa (Rejane et al. 2010), com características florísticas e estruturais típicas de cerrado (Castro et al. 1998). Apesar disso, algumas dessas áreas não pertencem ao limite oficial do bioma.

Flutuações climáticas causam alteração na distribuição das populações de árvores do Cerrado (Collevatti et al. 2003, 2009; Ramos et al. 2007). Diferenças máximas de temperatura entre os DB do Cerrado são de aproximadamente 8 으, e promovem importantes diferenças florísticas. Alterações entre +15 e -10 C C em relação à temperatura do presente foram observadas durante as mais recentes flutuações climáticas do Quaternário (9.000 antes do presente) para a região central do Cerrado, promovendo alterações importantes das comunidades vegetais ao longo dos últimos 30 mil anos (Ledru 1993). O DBNE está sobre uma das regiões de maior estabilidade climática ao longo das flutuações do Quaternário (Werneck et al. 2012), o que pode explicar a grande proporção de EPC observada para esse DB. Provavelmente, pela mesma razão, não são observadas espécies indicadoras compartilhadas com Caatinga, apesar da proximidade desse bioma. Futuras investigações sobre as relações históricas dessas espécies podem elucidar o papel dessa região na biota do Cerrado.

Os menores números de EPC estão nos DBS, DBSE, DBSW e DBN. Esses DB estão localizados nas extremidades do bioma, e, com exceção do DBSE, são as comunidades mais dissimilares, tendo aparecido na primeira divisão do agrupamento. Esses DB possuem valores extremos de temperatura mínima, que é a variável climática mais importante para a 
950

951

952

953

954

955

956

957

958

959

960

961

962

963

964

965

966

967

968

969

970

971

972

973

974

975

976

977

978

979

delimitação dos DB. Assim, é possível que essa variável estabeleça limites ótimos para ocorrência de maior número de EPC. Espécies compartilhadas com a Mata Atlântica são predominantes nos DB com menores temperaturas mínimas.

O DBS, a mais consistente e natural divisão do Cerrado (Ratter et al. 2003), tem grande influência da Mata Atlântica. Diversas das espécies indicadoras desse grupo já haviam sido propostas como características dessa região (Ratter et al. 2003). O DBSW, que também se diferenciou na primeira divisão do agrupamento, está localizado próximo ao Pantanal e ao Chaco. As espécies indicadoras desse grupo são comumente observadas em Florestas Estacionais distribuídas por todo o bioma (e. g. Kunz et al. 2008; Santos et al. 2007; Salis et al. 2004; Nascimento et al. 2004; Haidar et al. 2013). No entanto, a composição florística do cerrado desse DB é especialmente influenciada por essa vegetação, ou seja, as espécies características de Florestas Estacionais são mais comumente observadas nas fitofisionomias savânicas nesse DB do que nos demais.

Poucas espécies compartilhadas com a Amazônia suportam os DB, incluindo o DBCW, que possui grande extensão de fronteira com esse bioma. Diversos estudos demonstram menor influência da Amazônia sobre a vegetação do Cerrado em comparação com a Mata Atlântica (Heringer et al. 1977; Rizzini 1963b), inclusive considerando as matas de galeria (Méio et al. 2003). A transição Cerrado-Amazônia é clara, com evidências de turnover total das comunidades entre cerrado e floresta em pequenas distâncias (Marimon et al. 2006).

O DBC possui grande número de EPC e é característico de áreas altas do Cerrado. Muitos são os exemplos da ocorrência de espécies restrita a regiões de altitude (Alves \& Kolbek 2009; Echternacht et al. 2011; Gastauer et al. 2012; Nogueira et al. 2011; Silva 1997; Simon \& Proença 2000). A ocorrência dessas espécies nas áreas altas é atribuída às condições climáticas similares ao clima de períodos anteriores em regiões menos elevadas (Antonelli et al. 2010). Dessa maneira, as populações relictuais de áreas altas são insubstituíveis em termos de conservação, conferindo grande importância ao DBC. No entanto os esforços de conservação são mínimos, e a perda de habitat é elevada.

Diferenças ecológicas e históricas marcam a divisão das comunidades naturais da vegetação do cerrado. Os ecossistemas naturais atuam como substituto de biodiversidade (Noss 1996; Oliver et al. 2004). No entanto, para que os DB sejam usados como a primeira 
980

981

982

983

984

985

986

987

988

989

990

991

992

993

994

995

996

997

998

999

1000

1001

1002

1003

1004

1005

1006

1007

1008

1009

divisão das comunidades naturais do Cerrado, novos estudos são encorajados para avaliar a congruência entre os padrões observados para as árvores do cerrado e para outros grupos taxonômicos, como vertebrados, invertebrados e plantas de outros hábitos e fitofisionomias não avaliadas no presente estudo.

O primeiro diagnóstico do status dos DB mostrou grande discrepância nos esforços de conservação e dos padrões de ocupação das regiões com atributos biológicos distintos. Os resultados aqui apresentados são importantes para refinar o planejamento para a conservação da biodiversidade do Cerrado. Considerando as metas da Convenção da Diversidade Biológica (CDB), 17\% da área dos ecossistemas terrestres devem ser legalmente protegidos. No entanto, a área de remanescentes naturais dentro das unidades de conservação de proteção integral corresponde, a 3\% da área do Cerrado. Mesmo o DBNE, que apresenta a maior proporção de área protegida, está longe de ter seu território satisfatoriamente protegido quando consideramos apenas a área de unidades de conservação.

No entanto, o percentual de cobertura vegetal nativa não é dado suficiente para garantir a manutenção da biodiversidade, visto que, diversos fragmentos de remanescentes naturais encontram-se defaunados (Redford 1992). Diversas abordagens e técnicas consolidadas podem contribuir para esse processo. Modelos de nicho climático (Costa et al. 2010), técnicas de previsões de mudanças climáticas para diferentes grupos taxonômicos (Diniz-Filho et al. 2004, 2008; Margules et al. 2002; Marini et al. 2009; Pinto et al. 2008; Siqueira \& Peterson 2003), modelos de previsão de desmatamento (Faleiro et al. 2013) e estudos de fragmentação de habitat (Bianchi \& Haig 2012; Carvalho et al. 2009), aliados aos critérios do planejamento sistemático da conservação (Margules \& Pressey 2000), podem resultar na proposição de sistemas de áreas protegidas eficientes para a manutenção da biodiversidade.

Esse estudo representa um avanço no entendimento dos padrões biogeográficos para as espécies arbóreas do Cerrado. Novos distritos biogeográficos foram delimitados, mantendo os mesmos padrões observados em estudos anteriores. As espécies indicadoras desses DB e suas diferenças climáticas deram maior suporte às divisões encontradas, e a quantificação do desmatamento e do esforço de conservação são passos fundamentais para novas ações conservacionistas para o Cerrado. 


\section{AGRADECIMENTOS}

À CAPES, CNPq e Programa Ciência Sem Fronteira pelas bolsas de estudos concedidas a RDF. Ao CNPq pela bolsa de produtividade em pesquisa concedida a RBM. À Fundação O Boticário e ao Centro Agroecológico do Cerrado pelo apoio aos trabalhos em campo. À Rede ComCerrado e Royal Botanic Garden Edinburgh pelo fornecimento da estrutura de laboratório.

\section{REFERÊNCIAS}

Alves RJ V. \& Kolbek J, 2009. Can campo rupestre vegetation be floristically delimited based on vascular plant genera? Plant Ecology, 207(1):67-79.

Antonelli A, Verola CF, Parisod C \& Gustafsson a. LS, 2010. Climate cooling promoted the expansion and radiation of a threatened group of South American orchids (Epidendroideae: Laeliinae). Biological Journal of the Linnean Society, 100(3):597-607.

Arruda MB, Proença C, Rodrigues SC, et al., 2008. Ecorregiões, unidades de conservação e representatividade ecológica do bioma Cerrado. In SM Sano SP Almeida \& JF Ribeiro (eds.), Cerrado: ecologia e flora. Embrapa Ce ed., Brasília, DF.

Bianchi CA \& Haig SM, 2012. Deforestation Trends of Tropical Dry Forests in Central Brazil. 45(3):395-400.

Bongers F, Poorter L, Van-Rompaey RSAR \& Parren MPE, 1999. Distribution of twelve moist forest canopy tree species in Liberia and Côte d'Ivoire: response curves to a climatic gradient. Journal of Vegetation Science, 10(3):371-382.

Caetano S, Prado D, Pennington RT, et al., 2008. The history of Seasonally Dry Tropical Forests in eastern South America: inferences from the genetic structure of the tree Astronium urundeuva (Anacardiaceae). Molecular ecology, 17(13):3147-59.

Calenge C, 2006. The package "adehabitat" for the R software: A tool for the analysis of space and habitat use by animals. Ecological Modelling, 197(3-4):516-519.

Carvalho FM V, Marco P De \& Ferreira LG, 2009. The Cerrado into-pieces: Habitat fragmentation as a function of landscape use in the savannas of central Brazil. Biological Conservation, 142(7):1392-1403.

Castro AAJF, 1994. Comparação florístico-geográfica (Brasil) e fitossociologia (Piauíi - São Paulo) de amostras de cerrado. Instituto de Biologia. Campinas, SP: Universidade Estadual de Campinas.

Castro AAJF, Martins FR \& Fernandes AG, 1998. The woody flora of cerrado vegetation in the state of Piaui, northeastern Brazil. Edinburgh Journal of Botany, 55(03):455-472. 
Clements FE \& Shelford V, 1939. Bio-ecology. New York, USA / London, UK: John Wiley \& Sons / Chapman \& Hall, p. 425.

Cochrane TT, Sanchez LG, Azevedo LG, Porras JA \& Garver CL, 1985. Land in tropical America. EMBRAPA-CPAC.

Cole MMM, 1960. Cerrado , Caatinga and Pantanal: The Distribution and Origin of the Savanna Vegetation of Brazil. The Geographical Journal, 126(2):168-179.

Collevatti RG, Gonc S, Vieira RF \& Rabelo SG, 2009. Phylogeography and disjunct distribution in Lychnophora ericoides (Asteraceae), an endangered cerrado shrub species. Annals of Botany, 104(4):655 -664.

Collevatti RG, Grattapaglia D \& Hay JDV, 2003. Evidences for multiple maternal lineages of Caryocar brasiliense populations in the Brazilian Cerrado based on the analysis of chloroplast DNA sequences and microsatellite haplotype variation. Molecular ecology, 12(1):105-15.

Colli GR, 2005. As origens e a diversificação da herpetofauna do Cerrado. In A Scariot JC Sousasilva \& JM Felfili (eds.), Cerrado: Ecologia, Biodiversidade e Conservação. . 1st ed., Brasília, DF: MMA, p. 249-264.

Costa GC, Nogueira CC, Machado RB \& Colli GR, 2010. Sampling bias and the use of ecological niche modeling in conservation planning: a field evaluation in a biodiversity hotspot. Biodiversity and Conservation, 19(3):883-899.

Dapporto L, Ramazzotti M, Fattorini S, et al., 2013. Recluster: an Unbiased Clustering Procedure for Beta-Diversity Turnover. Ecography, 36(5):1070-1075.

Dice LR, 1943. The Biotic Provinces of North America. University of Michigan Press, p. 78.

Diniz-Filho JAF, Bini LM, Terribile LC, et al., 2008. Conservation planning: a macroecological approach using the endemic terrestrial vertebrates of the Brazilian Cerrado. Fauna \& Flora International, Oryx, 42(2):567-577.

Diniz-Filho JAF, Bini LM, Vieira CM, et al., 2004. Spatial patterns in species richness and priority areas for conservation of anurans in the Cerrado region, Central Brazil. Anphibia-Reptilia, 25(1):63-75.

Dinno A, 2014. Dunn's test of multiple comparisons using rank sums. R package version 1.2.0,

Dray S, Legendre P \& Blanchet G, 2011. Packfor: Forward Selection with permutation (Canoco p.46). R package version 0.0-8/r100., (http://r-forge.r-project.org/projects/sedar/).

Dufrêne $M$ \& Legendre $P, 1997$. Species assemblages and indicator species: the need for a flexible asymmetrical approach. Ecological monographs, 67(3):345-366. 
Echternacht L, Trovó M, Oliveira CT, Pirani JR \& Rubens J, 2011. Areas of endemism in the Espinhaço Range in Minas Gerais, Brazil. Flora - Morphology, Distribution, Functional Ecology of Plants, 206(9):782-791.

Eiten G, 1972. The Cerrado vegetation of Brazil. The Botanical Review, 38(2):201-341.

ESRI, 2011. ArcGIS Desktop. Redlands, CA: Environmental Systems Research Institute,

Faleiro F V., Machado RB \& Loyola RD, 2013. Defining spatial conservation priorities in the face of land-use and climate change. Biological Conservation, 158:248-257.

Felfili JM, Silva-Júnior MC, Sevilha AC, et al., 2004. Diversity, floristic and structural patterns of cerrado vegetation in Central Brazil. Plant Ecology, 175(1):37-46.

Furley P, 2006. Tropical savannas. Progress in Physical Geography, 30(1):105-121.

Furley P \& Ratter JA, 1988. Soil resources and plant communities of the central brazilian cerrado and their development. Journal of Biogeography, 15(1):97-108.

Gastauer M, Teixeira Braga Messias MC \& Alves Meira Neto JA, 2012. Floristic Composition, Species Richness and Diversity of Campo Rupestre Vegetation from the Itacolomi State Park, Minas Gerais, Brazil. Environment and Natural Resources Research, 2(3).

Gonçalves ET \& Souza AF, 2014. Floristic variation in ecotonal areas: Patterns, determinants and biogeographic origins of subtropical forests in South America. Austral Ecology, 39(1):122134.

Greve M, Lykke AM, Blach-Overgaard A \& Svenning J-C, 2011. Environmental and anthropogenic determinants of vegetation distribution across Africa. Global Ecology and Biogeography, 20(5):661-674.

Haffer J, 1969. Speciation in Amazonian Forest Birds. Science, 165(3889):131-137.

Haidar RF, Felfili JM, Pinto JRR, et al., 2013. Florestas estacionais e áreas de ecótono no estado do Tocantins, Brasil: parâmetros estruturais, classificação das fitofisionomias florestais e subsídios para conservação. Acta Amazonica, 43(530):261-290.

Heringer EP, Barroso GM, Rizzo JA \& Rizzini CT, 1977. A flora do Cerrado. In MG Ferri (ed.), IV Simpósio sobre o Cerrado. São Paulo, SP: Editora da Universidade de São Paulo, p. 211232.

Hijmans RJ, Cameron S, Parra J, Jones P \& Jarvis A. The WorldClim interpolated global terrestrial climate surfaces. (http://www.worldclim.org).

Holmgren $M$ \& Poorter L, 2007. Does a ruderal strategy dominate the endemic flora of the West African forests? Journal of Biogeography, 34(6):1100-1111.

IBGE. Mapa de Biomas do Brasil. Escala 1:5.000.000. (http://www.ibge.gov.br/home/presidencia/noticias/21052004biomas.shtm). 
IBGE, 2004b. Mapa de vegetação do Brasil. 2004 IEEE Region 10 Conference TENCON 2004. Brasília, DF: IBGE,

JBRJ. Lista de Espécies da Flora do Brasil. (http://floradobrasil.jbrj.gov.br/).

Klink CA \& Machado RB, 2005. Conservation of the Brazilian Cerrado. Conservation Biology, 19(3):707-713.

Kubota Y, Hirao T, Fujii S, Shiono T \& Kusumoto B, 2014. Beta diversity of woody plants in the Japanese archipelago: the roles of geohistorical and ecological processes. Journal of Biogeography, 41(7):1767-1276.

Kunz SH, Ivanauskas NM, Martins SV, Silva E \& Stefanello D, 2008. Aspectos florísticos e fitossociológicos de um trecho de Floresta Estacional Perenifólia na Fazenda Trairão, Bacia do rio das Pacas, Querência-MT. Acta Amazonica, 38(2):245-254.

Ledru M, 1993. Late Quaternary Environmental and Climatic Changes in Central Brazil. Quaternary Research, 39:90-98.

Lehmann CER, Anderson TM, Sankaran M, et al., 2014. Savanna Vegetation-Fire-Climate Relationships Differ Among Continents. Science, 343(January):548-552.

Lieberman M \& Lieberman D, 1994. Patterns of density and dispersion on forest trees. In LA McDade KS Bawa HA Hespenheide \& GS Hartshorn (eds.), La Selva - ecology and natural history of a neotropical rainforest. University of Chicago Press.

Margules CR \& Pressey RL, 2000. Systematic conservation planning. Nature, 405(May):243-253.

Margules CR, Pressey RL \& Williams PH, 2002. Representing biodiversity: data and procedures for identifying priority areas for conservation. Journal of biosciences, 27(2):309-26.

Marimon BS, De S. Lima E, Duarte TG, Chieregatto LC \& Ratter JA, 2006. Observations on the vegetation of northeastern Mato Grosso, Brazil. IV. An analysis of the cerrado-amazonian forest ecotone. Edinburgh Journal of Botany, 63(2-3):323-341.

Marini MÂ, Barbet-Massin M, Lopes LE \& Jiguet F, 2009. Major current and future gaps of Brazilian reserves to protect Neotropical savanna birds. Biological Conservation, 142(12):3039-3050.

McCune B \& Grace JB, 2002. Analysis of Ecological Communities. 1st ed., Gleneden Beach, Oregon: Oregon State University, p. 304.

Mckenzie D, Peterson DW, Peterson DL \& Seattle PET, 2003. Climatic and biophysical controls on conifer species distributions in mountain forests of Washington State, USA. Journal of Biogeography, 30(7):1093-1108.

Méio BB, Freitas C V., Jatobá L, et al., 2003. Influência da flora das florestas Amazônica e Atlântica na vegetação do cerrado sensu stricto. Revista Brasileira de Botânica, 26(4):437444. 
Meyer S, Augusto A, Garcia F \& Souza AP De, 2004. Comparison of similarity coefficients used for cluster analysis with dominant markers in maize (Zea mays L). Genetics and Molecular Biology, 27(1):83-91.

MMA, 2002. Biodiversidade brasileira: avaliação e identificação de áreas e ações prioritárias para a conservação utilização e repartição dos benefícios da biodiversidade nos biomas brasileiros. Brasília, DF.

MMA, 2006. Mapas de cobertura vegetal dos biomas brasileiros. Brasília, DF: Secretaria de Biodiversidade e Florestas, Ministério do Meio Ambiente-MA, (http://www.mma.gov.br).

Morrone JJ, 2001. Biogeografía de América Latina y el Caribe. Zaragoza: UNESCO, p. 148.

Nascimento ART, Felfili JM \& Meirelles M, 2004. Florística e estrutura da comunidade arbórea de um remanescente de Floresta Estacional Decidual de encosta , Monte Alegre , GO , Brasil. Terra, 18(3):659-669.

Nogueira CC, Ribeiro S, Costa G \& Colli GR, 2011. Vicariance and endemism in a Neotropical savanna hotspot: distribution patterns of Cerrado squamate reptiles. Journal of Biogeography, 38:1907-1922.

Noss RF, 1996. Ecosystems as conservation targets. Trends in Ecology \& Evolution, 11(8):351.

Novaes RML, De Lemos Filho JP, Ribeiro RA, et al., 2010. Phylogeography of Plathymenia reticulata (Leguminosae) reveals patterns of recent range expansion towards northeastern Brazil and southern Cerrados in Eastern Tropical South America. Molecular ecology, 19(5):985-998.

Oksanen J, Blanchet FG, Kindt R, et al., 2014. Community Ecology Package. R Package, version 2.0-10, (http://cran.r-project.org/web/packages/vegan/vegan.pdf).

Oliveira-Filho AT \& Ratter JA, 1995. A study of the origin of central Brazilian forests by the analysis of plant species distribution patterns. Edinburgh Journal of Botany, 52(02):141.

Oliver I, Holmes A, Dangerfield JM, et al., 2004. Land systems as surrogates for biodiversity in conservation planning. Ecological Applications, 14(2):485-503.

Oxford, 2010. A dictionary of Ecology. 4th ed., Oxford (United Kingdom), p. 418.

Pearson RG, 2006. Climate change and the migration capacity of species. Trends in Ecology and Evolution, 21(3):111-113.

Pinto MP, Diniz-Filho JAF, Bini LM, Blamires D \& Rangel TF, 2008. Biodiversity surrogate groups and conservation priority areas : birds of the Brazilian Cerrado. Diversity and Distributions, $14: 78-86$.

R Development Core Team, 2013. R: A language and environment for statistical computing. Vienna, Austria.: R Foundation for Statistical Computing, (http://www.r-project.org.). 
Ramos ACS, De Lemos-Filho JP, Lovato MB \& Lemos-Filho JP, 2009. Phylogeographical structure of the neotropical forest tree Hymenaea courbaril (Leguminosae: Caesalpinioideae) and its relationship with the Vicariant Hymenaea stigonocarpa from Cerrado. The Journal of heredity, 100(2):206-16.

Ramos ACS, Lemos-Filho JP, Ribeiro RA, Santos FR \& Lovato MB, 2007. Phylogeography of the tree Hymenaea stigonocarpa (Fabaceae: Caesalpinioideae) and the influence of quaternary climate changes in the Brazilian cerrado. Annals of botany, 100(6):1219-28.

Ratter JA, Bridgewater S, Atkinson R \& Ribeiro JF, 1996. Analysis of the floristic composition of the Brazilian cerrado vegetation II: Comparison of the woody vegetation of 98 areas. Edinburgh Journal of Botany, 53(02):153.

Ratter JA, Bridgewater S \& Ribeiro JF, 2003. Analysis of the Floristic Composition of the Brazilian Cerrado Vegetation III: Comparison of the Woody Vegetation of 376 Areas. Edinburgh Journal of Botany, 60(01):57-109.

Ratter JA, Bridgewater S, Ribeiro JF, et al. Analysis of the floristic composition of the Brazilian cerrado vegetation IV: Presentation of a Revised Data-Base of 367 Areas. (http://cerrado.rbge.org.uk/cerrado/download/download.php).

Ratter JA \& Dargie TCD, 1992. An analysis of the floristic composition of 26 cerrado ares in Brazil. Edinburgh Journal of Botany, 49(02):235.

Redford KH, 1992. The empty forest. BioScience, 42(6):412-422.

Rejane M, Mendes DA, Alberto A \& Farias J, 2010. Vascular flora of semi-arid region , São José do Piauí, state of Piauí , Brazil. Check list, 6(1):39-44.

Ribeiro JF \& Walter BMT, 2008. As principais fitofisionomias do bioma Cerrado. In SM Sano SP Almeida \& JF Ribeiro (eds.), Cerrado: ecologia e flora. . Brasília, DF: EMBRAPA, p. 151-212.

Rizzini CT, 1963a. Nota prévia sobre a divisão fitogeográfica do Brasil. Revista Brasileira de Geografia, 25(3):3-64.

Rizzini CT, 1963b. A flora do Cerrado. In , Simpósio sobre o Cerrado. São Paulo, SP: Editora da Universidade de São Paulo.

Rizzini CT \& Pinto MM, 1964. Áreas climático-vegetacionais do Brasil segundo os métodos de Thrnthwaite e Mohr. Revista Brasileira de Geografia, 26(4):523-547.

Rousseeuw P, Struyf A \& Hubert M, 2014. cluster: Cluster Analysis Extended Rousseeuw et al. (http://cran.r-project.org/web/packages/cluster/index.html).

Salgado-Labouriau ML, 2005. Alguns aspectos sobre a Paleoecologia dos Cerrados. In A Scariot JC Sousa-silva \& JM Felfili (eds.), Cerrado: Ecologia, Biodiversidade e Conservação. 1st ed., Brasília, DF: MMA, p. 107-119. 
Salis SM, Pereira M, Silva DA, et al., 2004. Fitossociologia de remanescentes de floresta estacional decidual em Corumbá , Estado do Mato Grosso do Sul , Brasil. (1895):671-684.

Santos RM Dos, Vieira F de A, Gusmão E \& Nunes YRF, 2007. Florística e estrutura de uma floresta estacional decidual no Parque Municipal da Sapucaia, Montes Claros (MG). Cerne, 13(3):248-256.

Sarmiento G, 1975. The Dry Plant Formations of South America and Their Floristic Connections. Journal of Biogeography, 2(4):233-251.

Scariot A \& Sevilha AC, 2005. Biodiversidade, estrutura e conservação de florestas estacionais deciduais no Cerrado. In A Scariot JC Sousa-Silva \& JM Felfili (eds.), Cerrado: ecologia, biodiversidade e conservação. MMA.

Silva JF, Farinas MR, Felfili JM \& Klink CA, 2006. Spatial heterogeneity, land use and conservation in the cerrado region of Brazil. Journal of Biogeography, 33:536-548.

Silva JMC, 1997. Endemic bird species and conservation in the Cerrado Region, South America. Biodiversity and Conservation, 6:435-450.

Simon MF, Grether R, De Queiroz LP, et al., 2009. Recent assembly of the Cerrado, a neotropical plant diversity hotspot, by in situ evolution of adaptations to fire. Proceedings of the National Academy of Sciences of the United States of America, 106(48):20359-64.

Simon MF \& Proença C, 2000. Phytogeographic patterns of Mimosa (Mimosoideae , Leguminosae) in the Cerrado biome of Brazil: an indicator genus of high-altitude centers of endemism? Biological Conservation, 96:279-296.

Siqueira MF \& Peterson AT, 2003. Consequences of Global climate change for geographic distributions of Cerrado tree species. Biota Neotropica, 3(2):1-14.

Smith LB, 1961. Origins of the flora of Southern Brazil. Contributions from the National Herbarium, 35:215-249.

Sokal RR \& Rohlf FJ, 1962. The comparison of dendrograms by objective methods. Taxon, 11:33-40.

Torello-Raventos M, Feldpausch TR, Veenendaal E, et al., 2013. On the delineation of tropical vegetation types with an emphasis on forest/savanna transitions. Plant Ecology \& Diversity, 6(1):101-137.

Udvardy MDF, 1975. A classification of the biogeographical provinces of the world. Morges, Switzerland: IUCN,

Unesco, 2000. Referencial teórico: o Cerrado, caracterização, ocupação e conservação. In , Vegetação no Distrito Federal: tempo e espaço.

Vanzolini $P$ \& Williams E, 1981. The vanishing refuge: a mechanism for ecogeographic speciation. Papéis Avulsos de Zoologia, 34(23):251-255. 
Veloso HP, 1964. Os grandes clímaces do Brasil. Memórias do Instituto Oswaldo Cruz, 62:203223.

Veloso HP \& Strang HE, 1970. Alguns aspectos fisionomicos da vegetação do Brasil. Memórias do Instituto Oswaldo Cruz, 68(1):9-76.

Venables WN \& Ripley BD, 2002. Modern Applied Statistics with S. New York: Springer,

Wallace AR, 1876. The geographical distribution of animals; with a study of the relations of living and extinct faunas as elucidating the past changes of the Earth's surface. London.

Werneck FP, Nogueira CC, Colli GR, Sites JW \& Costa GC, 2012. Climatic stability in the Brazilian Cerrado: implications for biogeographical connections of South American savannas, species richness and conservation in a biodiversity hotspot. Journal of Biogeography, 39(9):1695-1706. 


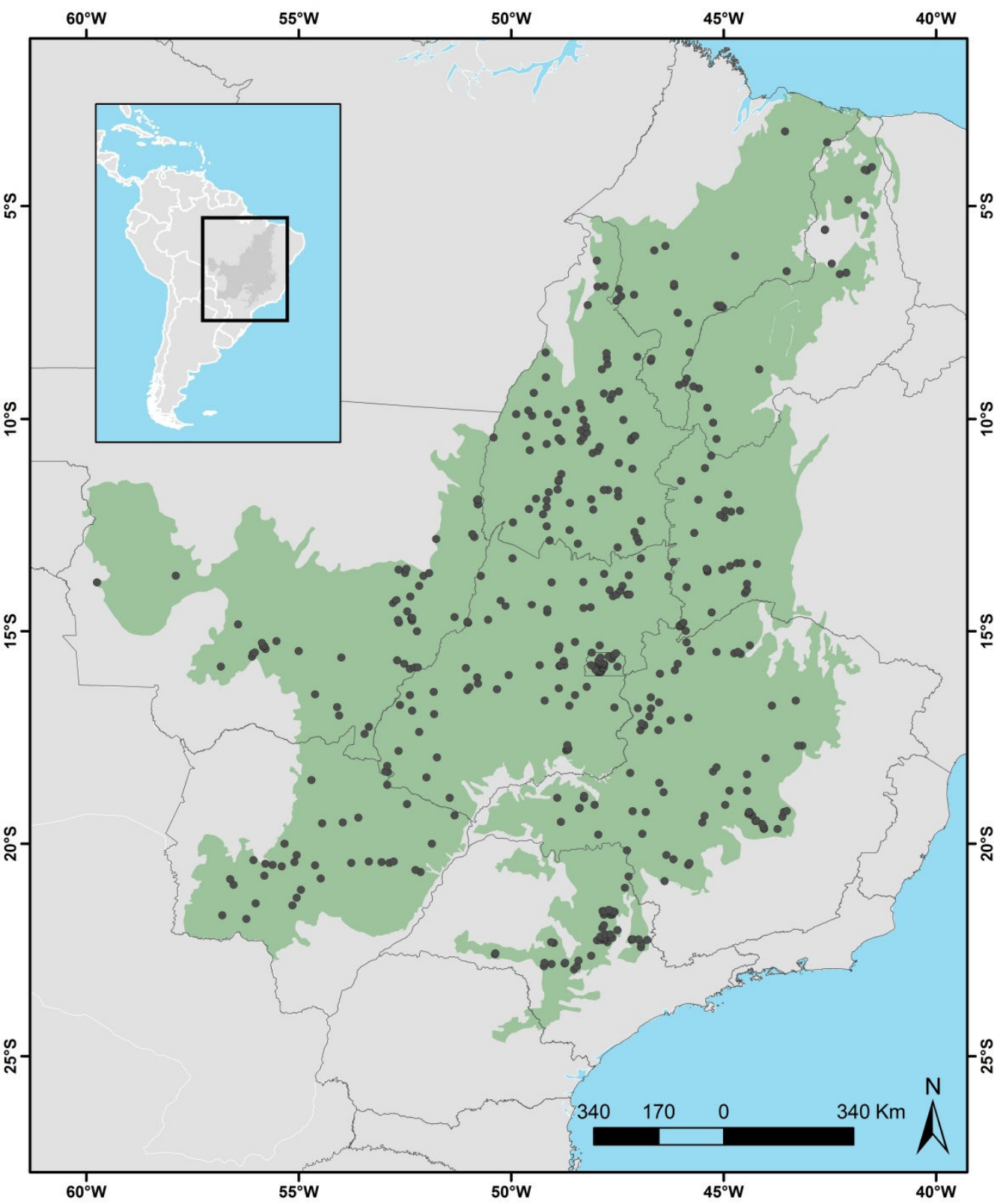

Figura 1. Área de estudo: 448 inventários e levantamentos florísticos em cerrado sentido 1263 amplo, usados para a delimitação dos Distritos Biogeográficos do Cerrado. 


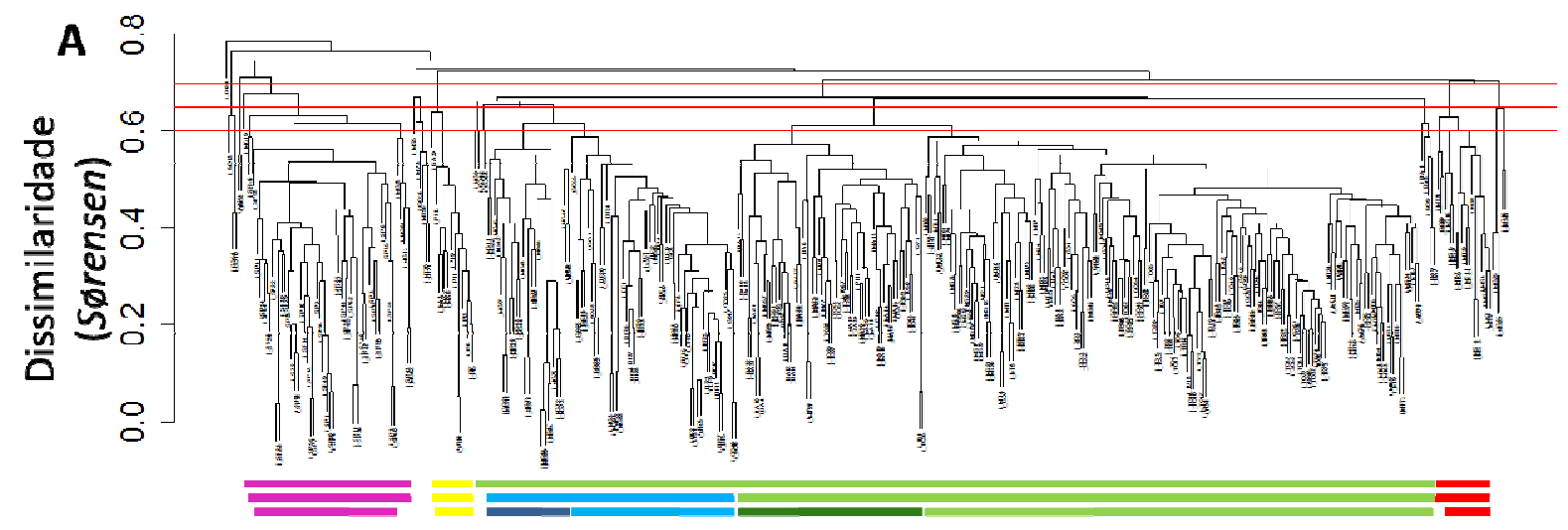

B

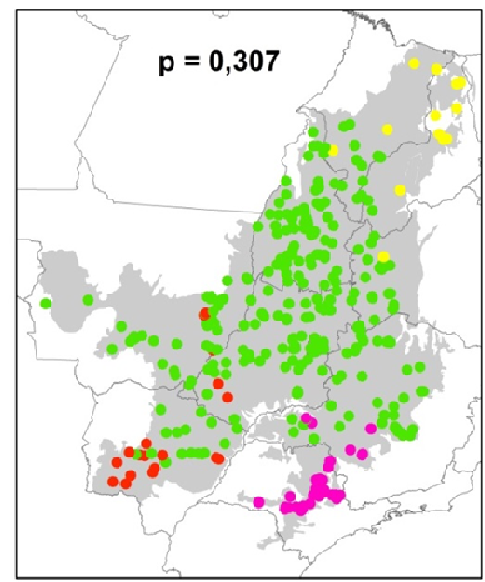

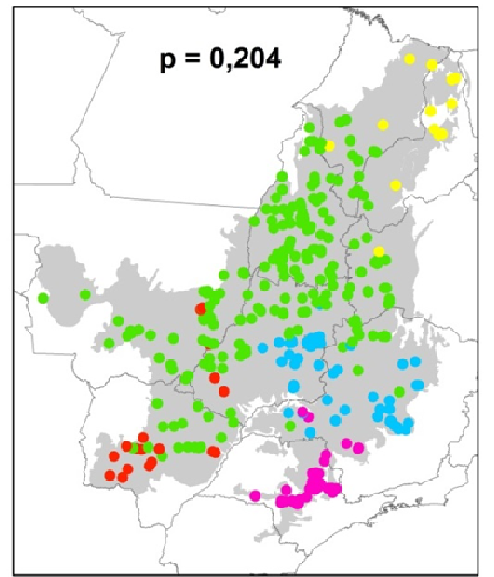

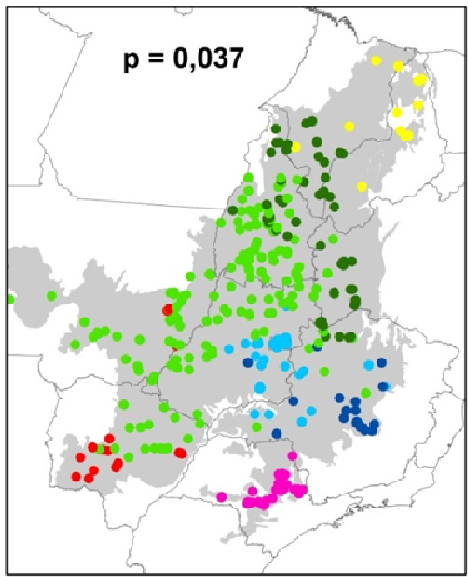

1265

1266

1267

1268

1269

1270

1271

Figura 2. (A) Análise de agrupamento (UPGMA) das comunidades arbóreas de cerrado sentido amplo e corte em diferentes níveis de dissimilaridade de Sørensen (0,70, 0,65 e 0,60). (B) Os Distritos Biogeográficos recuperados em cada nível de dissimilaridade. Na Terceira divisão: Amarelo = DB Norte; Vermelho=DB Sudoeste; Rosa = DB Sul; Verde claro = DB Centro-este; Verde escuro $=$ DB Nordeste; Azul claro $=$ DB Centro; Azul escuro $=$ DB Sudeste. O valor de $p$ é referente à média dos valores de $p$ para as espécies indicadoras em cada uma das divisões. 


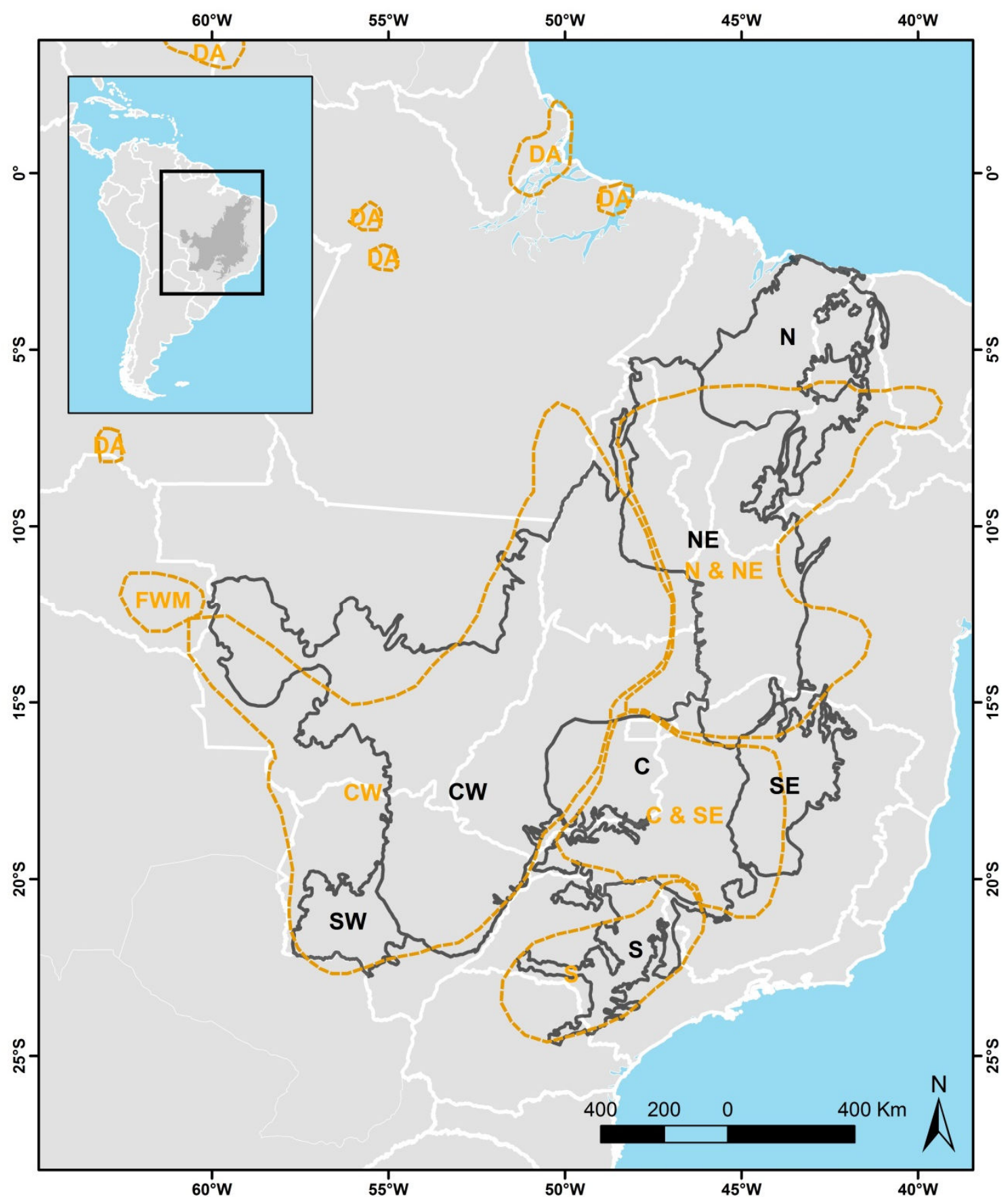

1274 Figura 3. As regiões biogeográficas propostas por Ratter et al. (2003) em traço pontilhado 1275 laranja, e os Distritos Biogeográficos Centro (C), Centro-oeste (CW), Norte (N), Nordeste (NE), 1276 Sul (S), Sudeste (SE) e Sudoeste (SW) propostos no presente estudo, em traço cinza. 


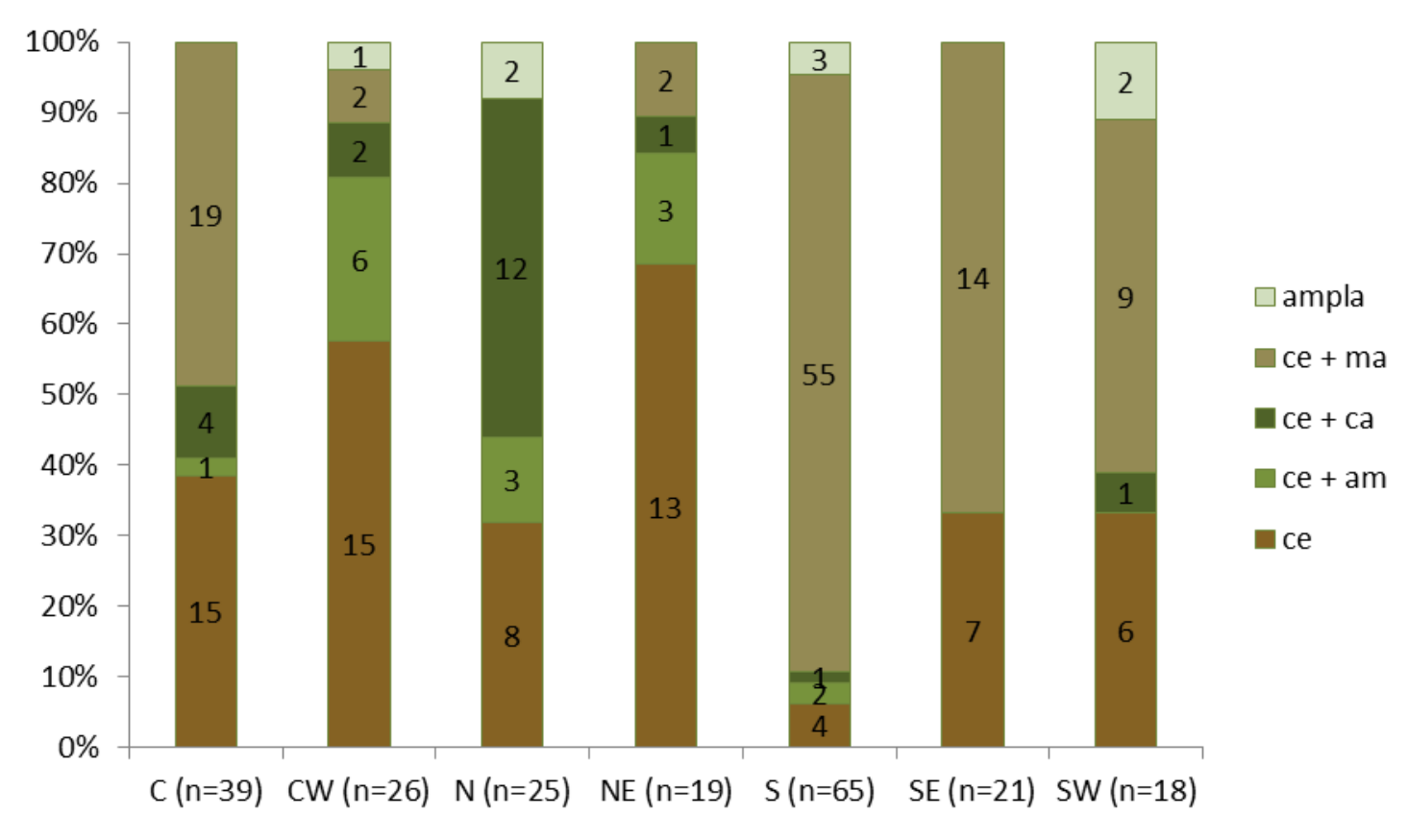

1279

Figura 4. Proporção e número de espécies indicadoras de cada bioma nos Distritos 1281 Biogeográficos Centro (C), Centro-oeste (CW), Norte (N), Nordeste (NE), Sul (S), Sudeste (SE) e 1282 Sudoeste (SW) do Cerrado. 

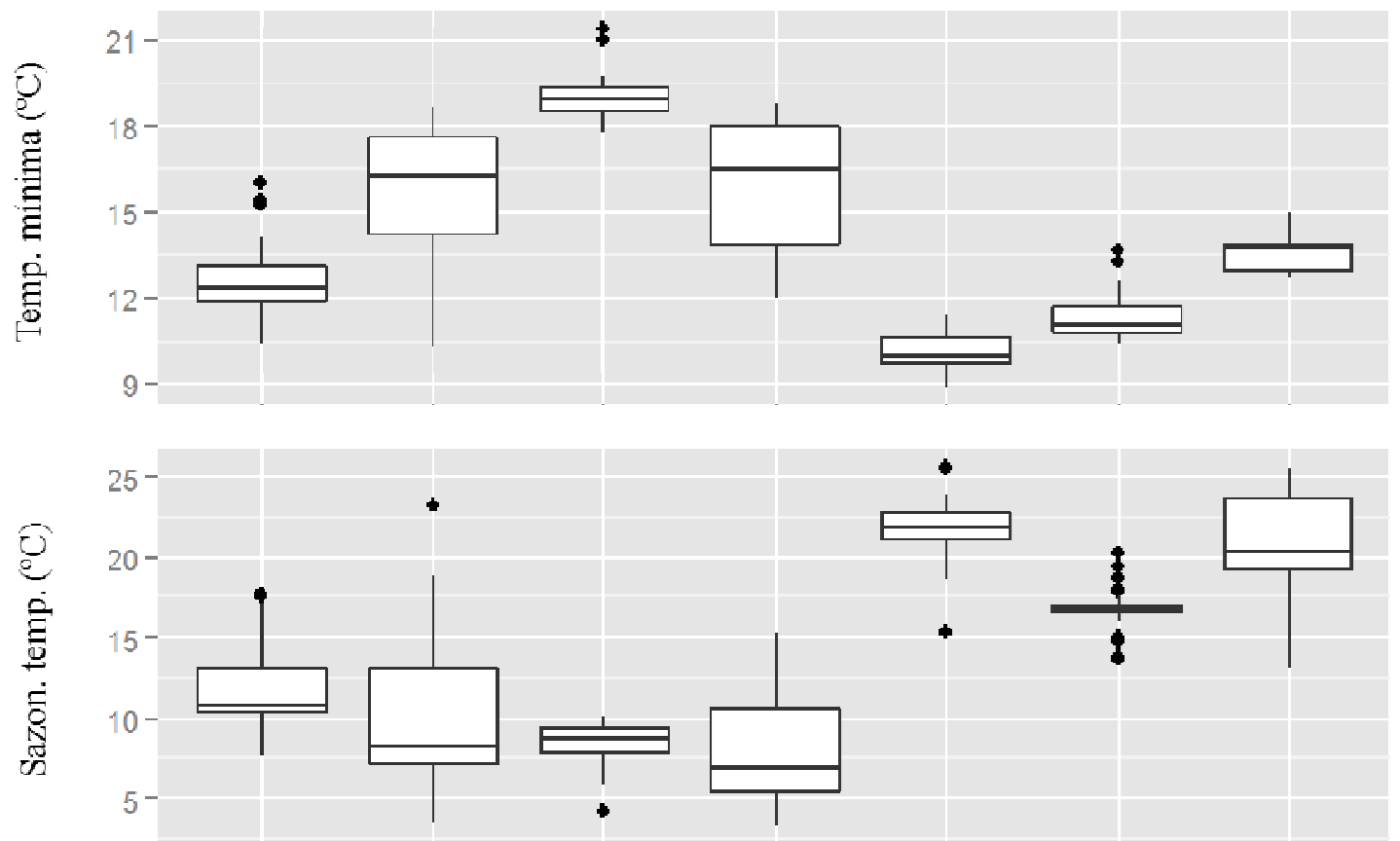

1285

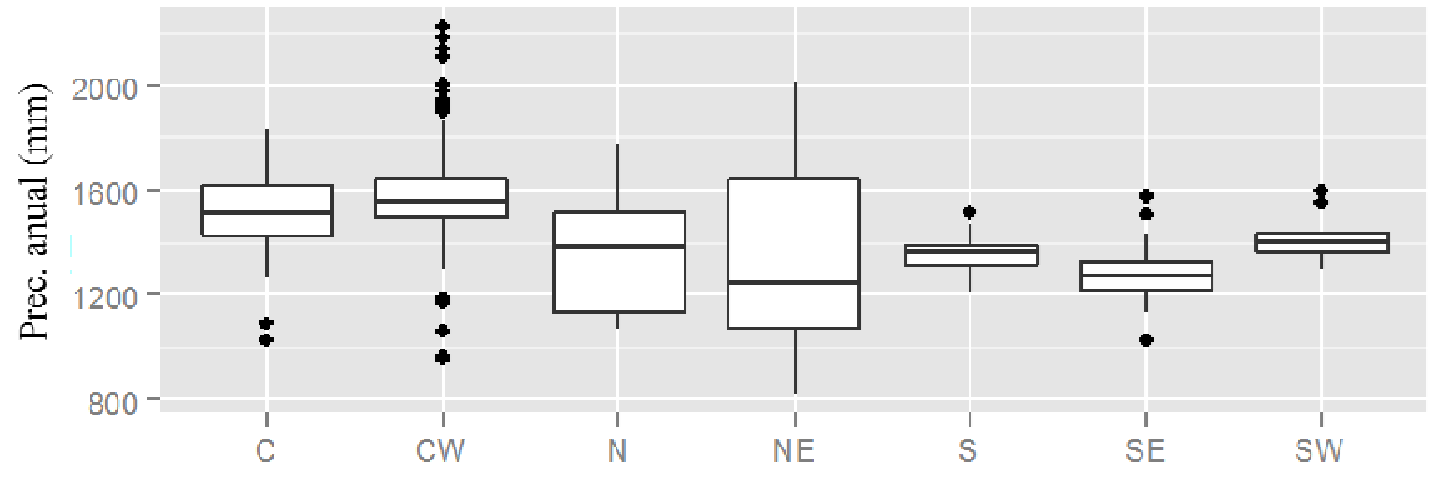

1286 Figura 5. Diferenciação das variáveis ambientais temperatura mínima, sazonalidade da 1287 temperatura e precipitação anual entre os Distritos Biogeográficos Centro (C), Centro-oeste 1288 (CW), Norte (N), Nordeste (NE), Sul (S), Sudeste (SE) e Sudoeste (SW) do Cerrado. 


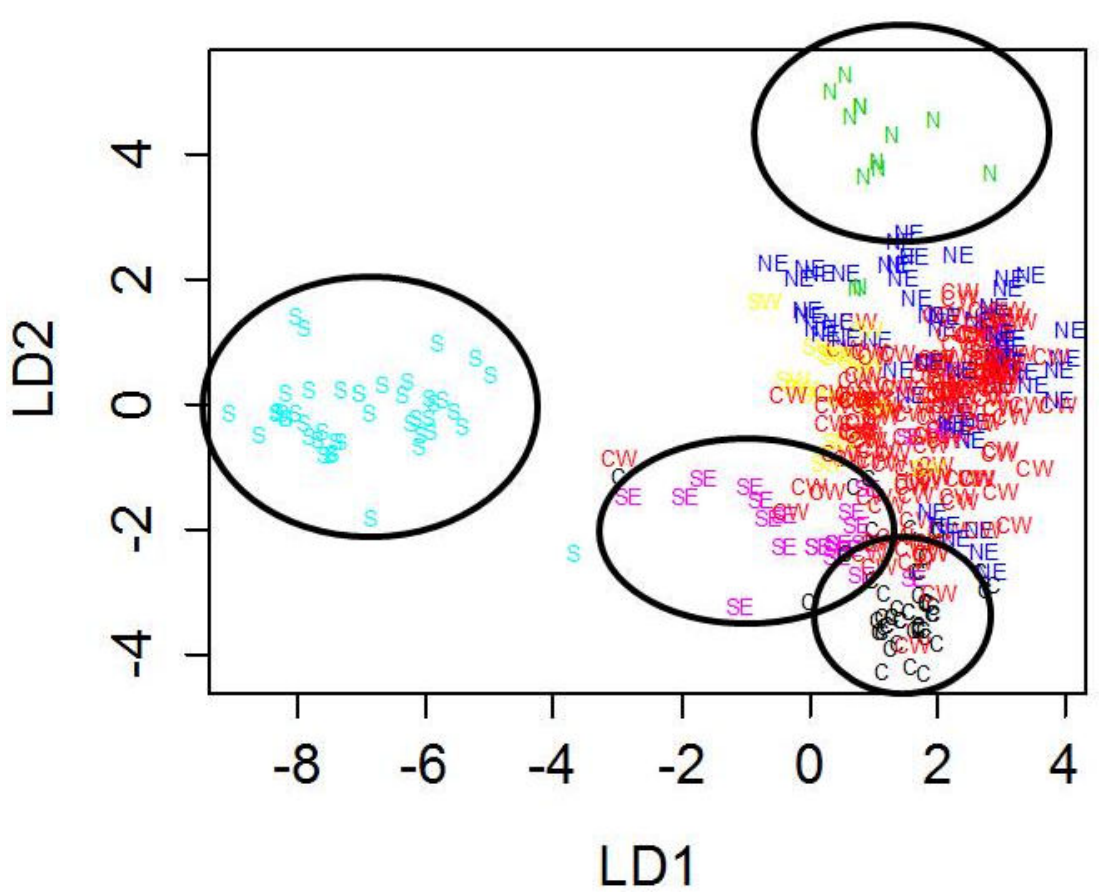

1291 Figura 6. Eixos um e dois da análise discriminante mostrando as principais separações climáticas 1292 dos Distritos Biogeográficos do Cerrado, onde $\mathrm{C}=$ Centro, CW = Centro-oeste; $\mathrm{N}=\mathrm{Norte}, \mathrm{NE}=$ 1293 Nordeste; S = Sul, SE = Sudeste e SW = Sudoeste. 


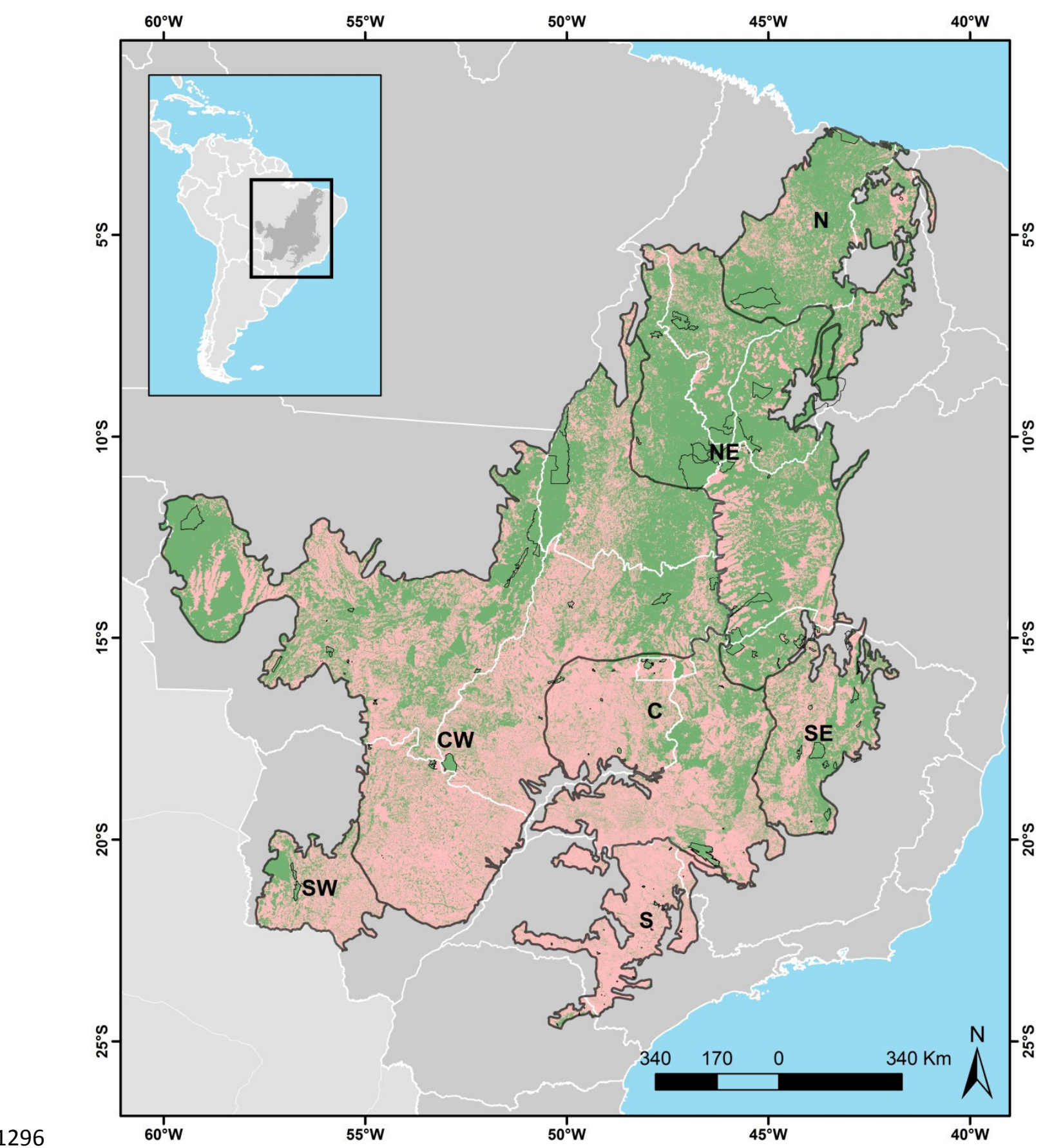

1297 Figura 7. Distritos Biogeográficos Centro (C), Centro-oeste (CW), Norte (N), Nordeste (NE), Sul 1298 (S), Sudeste (SE) e Sudoeste (SW) do Cerrado, com a cobertura vegetal remanescente (verde) e 1299 as unidades de conservação estaduais e federais de proteção integral. Fonte: MMA 2009. 
1302 Tabela 1. Análise de variância de matriz de similaridade entre os 448 inventários e 1303 levantamentos florísticos de vegetação lenhosa de cerrado sentido amplo, divididos em sete 1304 Distritos Biogeográficos no bioma Cerrado.

\begin{tabular}{lrrrrrr}
\hline & GL & $\begin{array}{c}\text { Soma dos } \\
\text { Quadrados }\end{array}$ & $\begin{array}{c}\text { Soma do } \\
\text { Quadrado } \\
\text { Médio }\end{array}$ & $\begin{array}{c}F- \\
\text { modelo }\end{array}$ & $R^{2}$ & $p$ \\
\hline Distritos Biogeográficos & 6 & 30,414 & 5,07 & 57,76 & 0,38 & 0,001 \\
Estrutura espacial & 55 & 17,731 & 0,32 & 3,67 & 0,22 & 0,001 \\
Intersecção & 161 & 18,815 & 0,12 & 1,33 & 0,23 & 0,001 \\
Resíduo & 150 & 13,164 & 0,09 & & 0,16 & \\
Total & 372 & 80,125 & 1 & & 1,00 & \\
\hline
\end{tabular}


1307 Tabela 2. Resultado da análise multivariada de variância (MANOVA) na detecção de diferenças 1308 entre variáveis climáticas dos Distritos Biogeográficos do Cerrado. A MANOVA foi significativa a 13090,05 de probabilidade $(F 8,11=361 ; p<0,001)$.

\begin{tabular}{lrrrrr}
\hline Variáveis & GL & $\begin{array}{c}\text { Soma dos } \\
\text { quadrados }\end{array}$ & $\begin{array}{c}\text { Soma dos } \\
\text { quadrados } \\
\text { médios }\end{array}$ & \multicolumn{1}{c}{$\mathbf{F}$} & p \\
\hline Altitude & 6 & 225.12 & 37.52 & 90.49 & $<0.001$ \\
Bio01 & 6 & 267.97 & 44.66 & 157.14 & $<0.001$ \\
Bio04 & 6 & 247.79 & 41.30 & $121.69<0.001$ \\
Bio05 & 6 & 264.45 & 44.07 & $149.98<0.001$ \\
Bio06 & 6 & 245.45 & 40.91 & $118.32<0.001$ \\
Bio12 & 6 & 97.172 & 16.19 & $21.57<0.001$ \\
Bio14 & 6 & 205.48 & 34.25 & 75.27 & $<0.001$ \\
Bio15 & 6 & 155.16 & 25.86 & $43.65<0.001$ \\
Bio20 & 6 & 191.48 & 31.91 & $64.71<0.001$ \\
Bio28 & 6 & 99.667 & 16.61 & $22.32<0.001$ \\
Bio31 & 6 & 203.52 & 33.92 & $73.69<0.001$ \\
\hline
\end{tabular}


1312 Tabela 3. Número de acertos (diagonál) e erros (coluna x linha) dos Distritos Biogeográficos 1313 Centro (C), Centro-oeste (CW), Norte (N), Nordeste (NE), Sul (S), Sudeste (SE) e Sudoeste (SW) 1314 do Cerrado, usando as variáveis climáticas selecionadas pela análise discriminante com critério 1315 de Uschi (temperatura mínima anual, sazonalidade da temperatura e precipitação total).

\begin{tabular}{lrrrrrrrr}
\hline DB & C & & CW & N & NE & S & SE & SW \\
\hline C & 49 & 1 & 0 & 0 & 0 & 3 & 0 \\
CW & 3 & 141 & 0 & 10 & 0 & 1 & 4 \\
N & 0 & 0 & 10 & 3 & 0 & 0 & 0 \\
NE & 0 & 10 & 0 & 50 & 0 & 0 & 0 \\
S & 0 & 0 & 0 & 0 & 46 & 0 & 0 \\
SE & 3 & 2 & 0 & 0 & 0 & 22 & 0 \\
SW & 0 & 5 & 0 & 0 & 0 & 0 & 10 \\
\hline
\end{tabular}

1316

1317 
Tabela 4. Comparação de variáveis climáticas entre Distritos Biogeográficos Centro (C), Centro-oeste (CW), Norte (N), Nordeste (NE), Sul (S), Sudeste (SE) e Sudoeste (SW) do Cerrado, usando o teste não paramétrico Dunn, conduzida a posteriori do teste de variância não paramétrico Kruskal-Wallis, significativo para todas as variáveis (Bio04: X26 = 246; $p<0.001 ;$ Bio06: X26 = 208; $p<0.001 ;$ Bio12: X26 = 117; $p<0.001$ ). As variáveis comparadas foram sazonalidade da precipitação (Bio04), temperatura mínima anual (Bio06) e precipitação anual (Bio12). Nível de significância adotado em 0,01 para todas as análises devido à correção de Bonferroni.

\begin{tabular}{|c|c|c|c|c|c|c|c|}
\hline & & C & CW & $\mathbf{N}$ & NE & $S$ & SE \\
\hline \multirow{3}{*}{ CW } & Bio06 & $X_{6}^{2}=-7.1 ; p<0.001$ & & & & & \\
\hline & Bio04 & $\mathrm{X}_{6}{ }_{6}=2.6 ; p=0.005$ & & & & & \\
\hline & Bio12 & $\mathrm{X}_{6}^{2}=-1.7 ; p=0.039$ & & & & & \\
\hline \multirow{3}{*}{$\mathbf{N}$} & Bio06 & $X_{6}^{2}=-7.1 ; p<0.001$ & $x_{6}^{2}=-3.7 ; p<0.001$ & & & & \\
\hline & Bio04 & $X^{2}=2.4 ; p=0.007$ & $\mathrm{X}_{6}{ }_{6}=1.2 ; \mathrm{p}=0.112$ & & & & \\
\hline & Bio12 & $X_{6}{ }_{6}=2.4 ; p=0.008$ & $\mathrm{X}_{6}{ }_{6}=3.5 ; p<0.001$ & & & & \\
\hline \multirow{3}{*}{ NE } & Bio06 & $X_{6}^{2}=-6.6 ; p<0.001$ & $X_{6}^{2}=-0.7 ; p=0.222$ & $\mathrm{X}_{6}{ }_{6}=3.1 ; p<0.001$ & & & \\
\hline & Bio04 & $\mathrm{X}_{6}{ }_{6}=4.8 ; \mathrm{p}<0.001$ & $\mathrm{X}_{6}{ }_{6}=3.3 ; \mathrm{p}<0.001$ & $\mathrm{X}_{6}^{2}=0.5 ; p=0.313$ & & & \\
\hline & Bio12 & $X_{6}^{2}=4.0 ; p<0.001$ & $\mathrm{X}_{6}{ }_{6}=6.8 ; \mathrm{p}<0.001$ & $X_{6}^{2}=0.04 ; p=0.48$ & & & \\
\hline \multirow{3}{*}{$\mathbf{S}$} & Bio06 & $X_{6}^{2}=4.2 ; p<0.001$ & $X_{6}^{2}=11.8 ; p<0.001$ & $\mathrm{X}_{6}{ }_{6}=9.7 ; p<0.001$ & $X_{6}^{2}=10.7 ; p<0.001$ & & \\
\hline & Bio04 & $X_{6}^{2}=-6.8 ; P<0.001$ & $X_{6}{ }_{6}=-10.6 ; p<0.001$ & $X^{2}{ }_{6}=-6.8 ; p<0.001$ & $\mathrm{X}_{6}{ }_{6}=-11.7 ; p<0.001$ & & \\
\hline & Bio12 & $\mathrm{X}_{6}{ }_{6}=4.8 ; p<0.001$ & $\mathrm{X}_{6}{ }_{6}=7.4 ; p<0.001$ & $X_{6}^{2}=0.7 ; p=0.240$ & $\mathrm{X}_{6}{ }_{6}=1.1 ; \mathrm{p}=0.145$ & & \\
\hline \multirow{3}{*}{ SE } & Bio06 & $\mathrm{X}_{6}{ }_{6}=1.8 ; \mathrm{p}=0.035$ & $X_{6}^{2}=7.5 ; p<0.001$ & $X_{6}^{2}=7.8 ; p<0.001$ & $\mathrm{X}_{6}{ }_{6}=7.2 ; \mathrm{p}<0.001$ & $\mathrm{X}_{6}{ }_{6}=-1.7 ; p=0.041$ & \\
\hline & Bio04 & $X_{6}^{2}=-3.6 ; p<0.001$ & $X_{6}^{2}=-6.0 ; p<0.001$ & $X^{2}=-4.7 ; p<0.001$ & $X_{6}{ }_{6}=-7.6 ; p<0.001$ & $\mathrm{X}_{6}{ }_{6}=2.2 ; \mathrm{p}=0.014$ & \\
\hline & Bio12 & $X_{6}^{2}=5.2 ;+<0.001$ & $X_{6}^{2}=7.3 ; p<0.001$ & $\mathrm{X}_{6}{ }_{6}=0.7 ; \mathrm{p}=0.240$ & $\mathrm{X}_{6}{ }_{6}=2.1 ; \mathrm{p}=0.017$ & $\mathrm{X}_{6}{ }_{6}=1.2 ; \mathrm{p}=0.123$ & \\
\hline \multirow{3}{*}{ sw } & Bio06 & $X_{6}^{2}=-1.4 ; p=0.084$ & $X_{6}^{2}=2.7 ; p=0.004$ & $\mathrm{X}_{6}^{2}=4.7 ; p<0.001$ & $\mathrm{X}_{6}{ }_{6}=2.9 ; p=0.002$ & $\mathrm{X}_{6}{ }_{6}=-4.2 ; p<0.001$ & $X_{6}^{2}=-2.6 ; p=0.005$ \\
\hline & Bio04 & $\mathrm{X}_{6}{ }_{6}=-4.0 ; \mathrm{p}<0.001$ & $\mathrm{X}_{6}{ }_{6}=-5.8 ; \mathrm{p}<0.001$ & $X_{6}^{2}=-5.1 ; p<0.001$ & $\mathrm{X}_{6}{ }_{6}=-7.2 ; p<0.001$ & $\mathrm{X}_{6}{ }_{6}=0.7 ; \mathrm{p}=0.242$ & $X_{6}^{2}=-1.0 ; p=0.158$ \\
\hline & Bio12 & $\mathrm{X}_{6}{ }_{6}=1.8 ; \mathrm{p}=0.030$ & $\mathrm{X}_{6}{ }_{6}=3.1 ; p=0.001$ & $X_{6}^{2}=-0.5 ; p=0.309$ & $\mathrm{X}_{6}{ }_{6}=-0.7 ; p=0.240$ & $X_{6}^{2}=-1.4 ; p=0.083$ & $X_{6}^{2}=-2.6 ; p=0.016$ \\
\hline
\end{tabular}

Tabela 5. Área total, área remanescente e Unidades de Conservação de Proteção Integral (UCPI) estaduais e federais dos Distritos Biogeográficos Centro (C), Centro-oeste (CW), Norte (N), Nordeste (NE), Sul (S), Sudeste (SE) e Sudoeste (SW) do Cerrado.

\begin{tabular}{|c|c|c|c|c|c|}
\hline $\begin{array}{c}\text { Distrito } \\
\text { Biogeográfico }\end{array}$ & Área (ha) & $\begin{array}{c}\text { Remanescente } \\
\text { (ha) }\end{array}$ & $\begin{array}{c}\% \\
\text { Remanescente }\end{array}$ & $\begin{array}{c}\text { Área } \\
\text { protegida }\end{array}$ & $\%$ UCPI \\
\hline
\end{tabular}




\begin{tabular}{lrrrrr}
\hline & & & & UCPI (ha) \\
& & & & & \\
\hline C & 29009660 & 9521989 & $33 \%$ & 315441,43 & $1,09 \%$ \\
CW & 90581860 & 44240628 & $49 \%$ & 1669189,38 & $1,84 \%$ \\
N & 20791870 & 14627241 & $70 \%$ & 613019,22 & $2,95 \%$ \\
NE & 43594120 & 32836214 & $75 \%$ & 2695550,23 & $6,18 \%$ \\
S & 8414493 & 886277 & $11 \%$ & 28668,62 & $0,34 \%$ \\
SE & 11635200 & 5574026 & $48 \%$ & 298770,39 & $2,57 \%$ \\
SW & 6703749 & 2351199 & $35 \%$ & 77304,02 & $1,15 \%$ \\
\hline
\end{tabular}

1325

1326

1327 


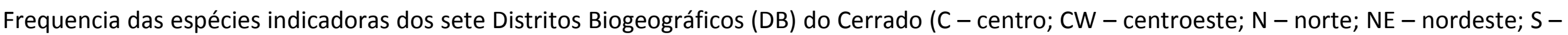
sul; SE - sudeste; SW - sudoeste) baseados na similaridade florística de 448 inventários e levantamentos florísticos da vegetação lenhosa realizada em cerrado sentido restrito e cerradão. O índice de associação (ISA) e sua significância foram calculados como proposto por Dufrêne \& Legendre (1997). Os biomas de ocorrência das espécies são Amazônia (Am), Caatinga (Ca), Cerrado (Ce), Mata Atlântica (MA), Pampas (Pam) e 1333 Pantal (Pan).

\begin{tabular}{|c|c|c|c|c|c|c|c|c|c|c|c|c|c|c|c|c|c|c|}
\hline \multirow[t]{2}{*}{ Família } & \multirow[t]{2}{*}{ Espécie } & \multirow[t]{2}{*}{ DB } & \multirow[t]{2}{*}{ ISA } & \multirow[t]{2}{*}{$\mathbf{p}$} & \multicolumn{7}{|c|}{$\begin{array}{l}\text { Biomas de ocorrência } \\
\text { predominante }\end{array}$} & \multicolumn{7}{|c|}{$\begin{array}{l}\text { Frequência das espécies nos Distritos } \\
\text { Biogeográficos }\end{array}$} \\
\hline & & & & & Am & $\mathrm{Ca}$ & $\mathrm{Ce}$ & $\mathrm{Ch}$ & MA & Pam & Pan & C & CW & $\mathbf{N}$ & NE & $\mathbf{S}$ & SE & sw \\
\hline Anacardiaceae & Anacardium occidentale & $\mathrm{N}$ & 0.384 & 0.001 & $x$ & & $x$ & & $x$ & & & 0.13 & 0.56 & 1 & 0.68 & 0.02 & 0.07 & 0.13 \\
\hline Anacardiaceae & Astronium fraxinifolium & SW & 0.237 & 0.001 & & $x$ & $x$ & & & & & 0.19 & 0.8 & 0.62 & 0.4 & 0 & 0.74 & 0.93 \\
\hline Anacardiaceae & Lithrea molleoides & SW & 0.212 & 0.002 & & & $x$ & & $x$ & & & 0 & 0.02 & 0 & 0 & 0.15 & 0.19 & 0.4 \\
\hline Anacardiaceae & Schinus terebinthifolius & SE & 0.34 & 0.001 & & & $x$ & & $x$ & & & 0 & 0.01 & 0 & 0 & 0.13 & 0.44 & 0 \\
\hline Annonaceae & Annona coriacea & $S$ & 0.174 & 0.03 & & & $x$ & & & & & 0.28 & 0.82 & 0.69 & 0.32 & 0.83 & 0.37 & 0.6 \\
\hline Annonaceae & Annona crassiflora & SE & 0.228 & 0.002 & & & $x$ & & $x$ & & & 0.62 & 0.43 & 0.15 & 0.6 & 0.7 & 0.89 & 0.07 \\
\hline Annonaceae & Cardiopetalum calophyllum & $\mathrm{C}$ & 0.094 & 0.035 & $x$ & & $x$ & & & & & 0.17 & 0.14 & 0 & 0 & 0 & 0 & 0 \\
\hline Annonaceae & Guatteria sellowiana & C & 0.07 & 0.037 & & & $x$ & & $x$ & & & 0.08 & 0.01 & 0 & 0 & 0 & 0 & 0 \\
\hline Annonaceae & Oxandra sessiliflora & $\mathrm{N}$ & 0.098 & 0.01 & & & $x$ & & & & & 0 & 0.04 & 0.15 & 0.05 & 0 & 0 & 0 \\
\hline Annonaceae & Xylopia aromatica & $\mathrm{S}$ & 0.186 & 0.019 & $x$ & & $x$ & & & & & 0.4 & 0.74 & 0.08 & 0.48 & 0.83 & 0.81 & 0.33 \\
\hline Apocynaceae & Aspidosperma macrocarpon & C & 0.226 & 0.001 & & & $x$ & & & & & 0.75 & 0.55 & 0.15 & 0.5 & 0 & 0.3 & 0.27 \\
\hline Apocynaceae & Aspidosperma multiflorum & $\mathrm{N}$ & 0.228 & 0.002 & & $x$ & $x$ & & & & & 0 & 0.11 & 0.31 & 0 & 0 & 0 & 0 \\
\hline Apocynaceae & Aspidosperma nobile & CW & 0.355 & 0.001 & & & $x$ & & & & & 0 & 0.52 & 0 & 0.03 & 0 & 0 & 0.2 \\
\hline Apocynaceae & Aspidosperma tomentosum & C & 0.191 & 0.008 & & & $x$ & & $x$ & & & 0.89 & 0.72 & 0 & 0.38 & 0.78 & 0.74 & 0.6 \\
\hline Apocynaceae & Himatanthus obovatus & NE & 0.243 & 0.001 & & & $x$ & & & & & 0.34 & 0.82 & 0.38 & 0.9 & 0.26 & 0.37 & 0.27 \\
\hline Aquifoliaceae & Ilex cerasifolia & $S$ & 0.076 & 0.04 & & & $x$ & & $x$ & & & 0 & 0.01 & 0 & 0 & 0.09 & 0 & 0 \\
\hline Araliaceae & Schefflera macrocarpa & C & 0.448 & 0.001 & & & $x$ & & $x$ & & & 0.94 & 0.14 & 0 & 0.02 & 0.11 & 0.78 & 0 \\
\hline Araliaceae & Schefflera vinosa & $\mathrm{S}$ & 0.588 & 0.001 & & & $x$ & & $x$ & & & 0.02 & 0.11 & 0 & 0.22 & 0.87 & 0.07 & 0 \\
\hline Asteraceae & Baccharis dracunculifolia & $S$ & 0.199 & 0.001 & & & $x$ & & $x$ & & & 0 & 0.01 & 0 & 0 & 0.26 & 0.07 & 0 \\
\hline
\end{tabular}




\begin{tabular}{|c|c|c|c|c|c|c|c|c|c|c|c|c|c|c|c|c|c|c|}
\hline \multirow[t]{2}{*}{ Família } & \multirow[t]{2}{*}{ Espécie } & \multirow[t]{2}{*}{ DB } & \multirow[t]{2}{*}{ ISA } & \multirow[t]{2}{*}{$\mathbf{p}$} & \multicolumn{7}{|c|}{$\begin{array}{l}\text { Biomas de ocorrência } \\
\text { predominante }\end{array}$} & \multicolumn{7}{|c|}{$\begin{array}{l}\text { Frequência das espécies nos Distritos } \\
\text { Biogeográficos }\end{array}$} \\
\hline & & & & & $\mathrm{Am}$ & $\mathrm{Ca}$ & $\mathrm{Ce}$ & $\mathrm{Ch}$ & MA & Pam & Pan & C & CW & $\mathbf{N}$ & NE & $\mathbf{S}$ & SE & SW \\
\hline Asteraceae & Eremanthus glomeratus & $\mathrm{C}$ & 0.615 & 0.001 & & $x$ & $x$ & & & & & 0.79 & 0.09 & 0 & 0.07 & 0 & 0.07 & 0 \\
\hline Asteraceae & Eremanthus goyazensis & $\mathrm{C}$ & 0.136 & 0.004 & & & $x$ & & & & & 0.17 & 0.03 & 0 & 0.02 & 0 & 0 & 0 \\
\hline Asteraceae & Gochnatia barrosoae & $\mathrm{S}$ & 0.435 & 0.001 & & & $x$ & & $x$ & & & 0 & 0 & 0 & 0 & 0.43 & 0 & 0 \\
\hline Asteraceae & Gochnatia polymorpha & $S$ & 0.154 & 0.004 & & & $x$ & & $x$ & & & 0.02 & 0 & 0 & 0.03 & 0.2 & 0 & 0 \\
\hline Asteraceae & Gochnatia pulchra & $\mathrm{S}$ & 0.487 & 0.001 & & & $x$ & & $x$ & & & 0 & 0 & 0 & 0 & 0.52 & 0.04 & 0 \\
\hline Asteraceae & Piptocarpha rotundifolia & $\mathrm{S}$ & 0.24 & 0.001 & & & $x$ & & $x$ & & & 0.72 & 0.33 & 0 & 0.27 & 0.83 & 0.63 & 0.07 \\
\hline Asteraceae & Vernonanthura ferruginea & SW & 0.347 & 0.001 & & & $x$ & & $x$ & & & 0.09 & 0.3 & 0 & 0.13 & 0.11 & 0.19 & 0.73 \\
\hline Asteraceae & Vernonanthura phosphorica & $\mathrm{S}$ & 0.096 & 0.026 & & & $x$ & & $x$ & & & 0.02 & 0.01 & 0 & 0 & 0.17 & 0.11 & 0 \\
\hline Bignoniaceae & Handroanthus impetiginosus & SW & 0.14 & 0.007 & & $x$ & $x$ & & $x$ & & & 0 & 0.14 & 0.31 & 0.02 & 0 & 0 & 0.33 \\
\hline Bignoniaceae & Jacaranda brasiliana & $\mathrm{N}$ & 0.23 & 0.001 & & $x$ & $x$ & & & & & 0 & 0.28 & 0.46 & 0.02 & 0 & 0.04 & 0.13 \\
\hline Bignoniaceae & Jacaranda caroba & $\mathrm{S}$ & 0.331 & 0.001 & & & $x$ & & $x$ & & & 0.04 & 0.03 & 0 & 0 & 0.46 & 0.04 & 0.07 \\
\hline Bignoniaceae & Jacaranda cuspidifolia & SW & 0.391 & 0.001 & & & $x$ & & $x$ & & & 0 & 0.12 & 0 & 0 & 0 & 0.07 & 0.53 \\
\hline Bignoniaceae & Zeyheria montana & SE & 0.326 & 0.001 & & & $x$ & & $x$ & & & 0.17 & 0.22 & 0.08 & 0.3 & 0.3 & 0.89 & 0.47 \\
\hline Boraginaceae & Cordia glabrata & $\mathrm{CW}$ & 0.204 & 0.001 & & $x$ & $x$ & & & & & 0 & 0.23 & 0 & 0.03 & 0 & 0 & 0 \\
\hline Burseraceae & Protium ovatum & $\mathrm{NE}$ & 0.111 & 0.019 & & & $x$ & & & & & 0.09 & 0.03 & 0 & 0.18 & 0 & 0 & 0 \\
\hline Calophyllaceae & Kielmeyera lathrophyton & NE & 0.258 & 0.001 & & & $x$ & & & & & 0.09 & 0.2 & 0 & 0.43 & 0 & 0 & 0 \\
\hline Calophyllaceae & Kielmeyera rubriflora & $\mathrm{CW}$ & 0.132 & 0.04 & & & $x$ & & & & & 0.17 & 0.38 & 0 & 0.05 & 0.37 & 0.07 & 0.07 \\
\hline Calophyllaceae & Kielmeyera speciosa & $\mathrm{C}$ & 0.442 & 0.001 & & & $x$ & & & & & 0.66 & 0.17 & 0 & 0.02 & 0 & 0.07 & 0.07 \\
\hline Caryocaraceae & Caryocar brasiliense & SE & 0.208 & 0.003 & & & $x$ & & $x$ & & & 0.89 & 0.57 & 0.15 & 0.38 & 0.74 & 0.93 & 0.47 \\
\hline Caryocaraceae & Caryocar coriaceum & $\mathrm{N}$ & 0.614 & 0.001 & & $x$ & $x$ & & & & & 0.02 & 0.16 & 0.85 & 0.12 & 0.02 & 0 & 0 \\
\hline Caryocaraceae & Caryocar cuneatum & $\mathrm{NE}$ & 0.301 & 0.001 & $x$ & & $x$ & & & & & 0 & 0.11 & 0.08 & 0.43 & 0 & 0 & 0 \\
\hline Celastraceae & Plenckia populnea & $\mathrm{C}$ & 0.245 & 0.001 & & & $x$ & & $x$ & & & 0.81 & 0.29 & 0 & 0.12 & 0.72 & 0.56 & 0.2 \\
\hline Celastraceae & Salacia crassifolia & $\mathrm{C}$ & 0.252 & 0.002 & & & $x$ & & & & & 0.74 & 0.53 & 0.08 & 0.47 & 0 & 0.33 & 0 \\
\hline Chrysobalanaceae & Couepia grandiflora & $S$ & 0.22 & 0.004 & & & $x$ & & $x$ & & & 0.45 & 0.6 & 0.08 & 0.5 & 0.78 & 0.37 & 0 \\
\hline Chrysobalanaceae & Hirtella ciliata & $\mathrm{N}$ & 0.375 & 0.001 & & $x$ & $x$ & & & & & 0 & 0.11 & 0.77 & 0.7 & 0 & 0 & 0 \\
\hline Chrysobalanaceae & Licania gardneri & $\mathrm{CW}$ & 0.107 & 0.02 & & & $x$ & & & & & 0 & 0.11 & 0 & 0 & 0 & 0 & 0 \\
\hline Chrysobalanaceae & Licania humilis & $\mathrm{S}$ & 0.382 & 0.001 & & & $x$ & & $x$ & & & 0.15 & 0.16 & 0 & 0 & 0.59 & 0 & 0 \\
\hline Chrysobalanaceae & Licania sclerophylla & $\mathrm{CW}$ & 0.082 & 0.029 & $x$ & & $x$ & & & & & 0 & 0.08 & 0 & 0 & 0 & 0 & 0 \\
\hline
\end{tabular}




\begin{tabular}{|c|c|c|c|c|c|c|c|c|c|c|c|c|c|c|c|c|c|c|}
\hline \multirow[t]{2}{*}{ Família } & \multirow[t]{2}{*}{ Espécie } & \multirow[t]{2}{*}{ DB } & \multirow[t]{2}{*}{ ISA } & \multirow[t]{2}{*}{$\mathbf{p}$} & \multicolumn{7}{|c|}{$\begin{array}{l}\text { Biomas de ocorrência } \\
\text { predominante }\end{array}$} & \multicolumn{7}{|c|}{$\begin{array}{l}\text { Frequência das espécies nos Distritos } \\
\text { Biogeográficos }\end{array}$} \\
\hline & & & & & Am & $\mathrm{Ca}$ & $\mathrm{Ce}$ & $\mathrm{Ch}$ & MA & Pam & Pan & C & CW & $\mathbf{N}$ & NE & $\mathbf{S}$ & SE & sw \\
\hline Clusiaceae & Platonia insignis & $\mathrm{N}$ & 0.227 & 0.001 & $x$ & & $x$ & & & & & 0 & 0.03 & 0.31 & 0.08 & 0 & 0 & 0 \\
\hline Combretaceae & Buchenavia tetraphylla & $\mathrm{N}$ & 0.118 & 0.006 & $x$ & $x$ & $x$ & & & & & 0 & 0.01 & 0.15 & 0.03 & 0 & 0 & 0 \\
\hline Combretaceae & Buchenavia tomentosa & CW & 0.32 & 0.001 & & & $x$ & & & & & 0 & 0.57 & 0 & 0.12 & 0 & 0 & 0.33 \\
\hline Combretaceae & Combretum duarteanum & $\mathrm{N}$ & 0.366 & 0.001 & & $x$ & $x$ & & & & & 0 & 0.04 & 0.46 & 0.08 & 0 & 0 & 0 \\
\hline Combretaceae & Combretum mellifluum & $\mathrm{N}$ & 0.401 & 0.001 & & $x$ & $x$ & & & & & 0 & 0.01 & 0.62 & 0.3 & 0.02 & 0 & 0 \\
\hline Combretaceae & Terminalia argentea & SW & 0.281 & 0.001 & & & $x$ & & $x$ & & & 0.26 & 0.7 & 0 & 0.08 & 0.2 & 0.56 & 0.87 \\
\hline Combretaceae & Terminalia fagifolia & $\mathrm{N}$ & 0.217 & 0.001 & & $x$ & $x$ & & & & & 0.13 & 0.15 & 0.69 & 0.68 & 0.02 & 0.26 & 0.27 \\
\hline Connaraceae & Rourea induta & $\mathrm{CW}$ & 0.242 & 0.002 & & & $x$ & & & & & 0.28 & 0.71 & 0 & 0.55 & 0.09 & 0.26 & 0.2 \\
\hline Dichapetalaceae & Tapura amazonica & $\mathrm{CW}$ & 0.099 & 0.025 & $x$ & & $x$ & & & & & 0.04 & 0.14 & 0 & 0.02 & 0 & 0 & 0 \\
\hline Dilleniaceae & Curatella americana & $\mathrm{CW}$ & 0.208 & 0.001 & & & $x$ & & & & & 0.21 & 0.9 & 0.85 & 0.5 & 0 & 0.78 & 0.67 \\
\hline Ebenaceae & Diospyros coccolobifolia & $\mathrm{CW}$ & 0.12 & 0.022 & & & $x$ & & $x$ & & & 0 & 0.18 & 0 & 0.08 & 0 & 0 & 0 \\
\hline Erythroxylaceae & Erythroxylum cuneifolium & $\mathrm{S}$ & 0.329 & 0.001 & & & $x$ & & $x$ & & & 0 & 0 & 0 & 0 & 0.39 & 0.07 & 0 \\
\hline Erythroxylaceae & Erythroxylum daphnites & SE & 0.162 & 0.011 & & & $x$ & & & & & 0.08 & 0.21 & 0 & 0 & 0 & 0.41 & 0.33 \\
\hline Erythroxylaceae & Erythroxylum deciduum & $\mathrm{C}$ & 0.238 & 0.001 & & & $x$ & & $x$ & & & 0.66 & 0.36 & 0 & 0.25 & 0.24 & 0.19 & 0.13 \\
\hline Erythroxylaceae & Erythroxylum pelleterianum & $\mathrm{S}$ & 0.168 & 0.004 & & & $x$ & & $x$ & & & 0 & 0.01 & 0 & 0 & 0.17 & 0 & 0 \\
\hline Erythroxylaceae & Erythroxylum tortuosum & $\mathrm{C}$ & 0.28 & 0.001 & & & $x$ & & $x$ & & & 0.81 & 0.34 & 0 & 0.08 & 0.52 & 0.59 & 0 \\
\hline Euphorbiaceae & Cnidoscolus vitifolius & $\mathrm{CW}$ & 0.107 & 0.021 & & $x$ & $x$ & & & & & 0 & 0.11 & 0 & 0 & 0 & 0 & 0 \\
\hline Fabaceae & Anadenanthera peregrina & $\mathrm{S}$ & 0.434 & 0.001 & & & $x$ & & $x$ & & & 0.04 & 0.16 & 0 & 0.02 & 0.8 & 0.07 & 0.4 \\
\hline Fabaceae & Andira anthelmia & $\mathrm{S}$ & 0.093 & 0.019 & & & $x$ & & $x$ & & & 0 & 0.02 & 0 & 0 & 0.11 & 0 & 0 \\
\hline Fabaceae & Andira cordata & $\mathrm{NE}$ & 0.277 & 0.001 & & & $x$ & & & & & 0 & 0.01 & 0 & 0.28 & 0 & 0 & 0 \\
\hline Fabaceae & Andira cujabensis & $\mathrm{CW}$ & 0.355 & 0.001 & & & $x$ & & & & & 0 & 0.65 & 0 & 0.35 & 0 & 0 & 0.2 \\
\hline Fabaceae & Andira vermifuga & $\mathrm{NE}$ & 0.212 & 0.005 & & & $x$ & & & & & 0.4 & 0.66 & 0.46 & 0.8 & 0.07 & 0.04 & 0.6 \\
\hline Fabaceae & Apuleia leiocarpa & $\mathrm{CW}$ & 0.08 & 0.044 & & & $x$ & & $x$ & & & 0 & 0.09 & 0 & 0.02 & 0 & 0 & 0 \\
\hline Fabaceae & Bauhinia forficata & SE & 0.259 & 0.001 & & & $x$ & & $x$ & & & 0 & 0 & 0 & 0 & 0 & 0.26 & 0 \\
\hline Fabaceae & Bauhinia holophylla & $S$ & 0.224 & 0.001 & & & $x$ & & $x$ & & & 0 & 0 & 0 & 0 & 0.28 & 0.07 & 0 \\
\hline Fabaceae & Bauhinia rufa & $S$ & 0.256 & 0.001 & & & $x$ & & $x$ & & & 0.04 & 0.28 & 0 & 0 & 0.59 & 0.11 & 0.33 \\
\hline Fabaceae & Copaifera coriacea & $\mathrm{N}$ & 0.205 & 0.001 & & $x$ & $x$ & & & & & 0 & 0.01 & 0.23 & 0.02 & 0 & 0 & 0 \\
\hline Fabaceae & Copaifera langsdorffii & $\mathrm{S}$ & 0.16 & 0.034 & & & $x$ & & $x$ & & & 0.34 & 0.58 & 0.31 & 0.25 & 0.72 & 0.63 & 0.4 \\
\hline
\end{tabular}




\begin{tabular}{|c|c|c|c|c|c|c|c|c|c|c|c|c|c|c|c|c|c|c|}
\hline \multirow[t]{2}{*}{ Família } & \multirow[t]{2}{*}{ Espécie } & \multirow[t]{2}{*}{ DB } & \multirow[t]{2}{*}{ ISA } & \multirow[t]{2}{*}{$\mathbf{p}$} & \multicolumn{7}{|c|}{$\begin{array}{l}\text { Biomas de ocorrência } \\
\text { predominante }\end{array}$} & \multicolumn{7}{|c|}{$\begin{array}{l}\text { Frequência das espécies nos Distritos } \\
\text { Biogeográficos }\end{array}$} \\
\hline & & & & & Am & $\mathrm{Ca}$ & $\mathrm{Ce}$ & $\mathrm{Ch}$ & MA & Pam & Pan & C & CW & $\mathbf{N}$ & NE & $\mathbf{S}$ & SE & sw \\
\hline Fabaceae & Copaifera martii & NE & 0.184 & 0.003 & $x$ & & $x$ & & & & & 0 & 0.13 & 0.23 & 0.43 & 0.02 & 0 & 0.2 \\
\hline Fabaceae & Dalbergia miscolobium & $\mathrm{S}$ & 0.169 & 0.032 & & & $x$ & & $x$ & & & 0.79 & 0.43 & 0.62 & 0.45 & 0.8 & 0.67 & 0.07 \\
\hline Fabaceae & Dimorphandra gardneriana & $\mathrm{N}$ & 0.649 & 0.001 & & $x$ & $x$ & & & & & 0 & 0.16 & 0.85 & 0.1 & 0 & 0 & 0 \\
\hline Fabaceae & Dipteryx alata & SW & 0.314 & 0.001 & & & $x$ & & & & & 0.08 & 0.58 & 0.15 & 0 & 0.02 & 0.41 & 0.8 \\
\hline Fabaceae & Diptychandra aurantiaca & $S$ & 0.112 & 0.029 & & $x$ & $x$ & & & & & 0 & 0.19 & 0 & 0.03 & 0.28 & 0 & 0.2 \\
\hline Fabaceae & Leptolobium elegans & $\mathrm{S}$ & 0.442 & 0.001 & & & $x$ & & $x$ & & & 0.09 & 0.09 & 0 & 0.02 & 0.87 & 0.04 & 0.6 \\
\hline Fabaceae & Luetzelburgia auriculata & $\mathrm{N}$ & 0.518 & 0.001 & & $x$ & $x$ & & & & & 0 & 0 & 0.54 & 0 & 0.02 & 0 & 0 \\
\hline Fabaceae & Luetzelburgia praecox & $\mathrm{CW}$ & 0.1 & 0.018 & & & $x$ & & & & & 0 & 0.15 & 0.08 & 0 & 0 & 0 & 0 \\
\hline Fabaceae & Machaerium acutifolium & $\mathrm{S}$ & 0.182 & 0.021 & & & $x$ & & $x$ & & & 0.28 & 0.76 & 0.77 & 0.55 & 0.89 & 0.37 & 0.73 \\
\hline Fabaceae & Machaerium opacum & SE & 0.322 & 0.001 & & & $x$ & & & & & 0.72 & 0.38 & 0.08 & 0.23 & 0 & 0.85 & 0 \\
\hline Fabaceae & Machaerium villosum & $\mathrm{S}$ & 0.135 & 0.01 & & & $x$ & & $x$ & & & 0.04 & 0 & 0 & 0 & 0.24 & 0.15 & 0 \\
\hline Fabaceae & Martiodendron mediterraneum & $\mathrm{N}$ & 0.323 & 0.001 & $x$ & & $x$ & & & & & 0 & 0.01 & 0.38 & 0.07 & 0 & 0 & 0 \\
\hline Fabaceae & Mimosa claussenii & $\mathrm{C}$ & 0.223 & 0.002 & & & $x$ & & & & & 0.34 & 0.07 & 0 & 0.03 & 0 & 0.07 & 0 \\
\hline Fabaceae & Mimosa laticifera & SE & 0.093 & 0.032 & & & $x$ & & & & & 0 & 0.09 & 0 & 0 & 0 & 0.15 & 0 \\
\hline Fabaceae & Parkia platycephala & $\mathrm{N}$ & 0.646 & 0.001 & & $x$ & $x$ & & & & & 0 & 0.08 & 1 & 0.47 & 0 & 0 & 0 \\
\hline Fabaceae & Plathymenia reticulata & $\mathrm{N}$ & 0.203 & 0.003 & & & $x$ & & & & & 0.53 & 0.67 & 0.92 & 0.75 & 0.41 & 0.44 & 0.47 \\
\hline Fabaceae & Pterodon emarginatus & $\mathrm{NE}$ & 0.356 & 0.001 & & & $x$ & & & & & 0.08 & 0.25 & 0.23 & 0.72 & 0.07 & 0.11 & 0 \\
\hline Fabaceae & Pterodon pubescens & $\mathrm{C}$ & 0.216 & 0.001 & & & $x$ & & $x$ & & & 0.58 & 0.42 & 0 & 0.15 & 0.22 & 0.15 & 0.07 \\
\hline Fabaceae & Senna rugosa & $\mathrm{S}$ & 0.231 & 0.001 & & & $x$ & & $x$ & & & 0.06 & 0.03 & 0.15 & 0.02 & 0.48 & 0.26 & 0 \\
\hline Fabaceae & Stryphnodendron adstringens & SE & 0.26 & 0.001 & & & $x$ & & $x$ & & & 0.85 & 0.23 & 0 & 0.17 & 0.57 & 0.89 & 0.33 \\
\hline Fabaceae & Stryphnodendron coriaceum & $\mathrm{N}$ & 0.558 & 0.001 & & & $x$ & & & & & 0 & 0.09 & 0.85 & 0.35 & 0 & 0 & 0 \\
\hline Fabaceae & Stryphnodendron polyphyllum & $\mathrm{S}$ & 0.252 & 0.001 & & & $x$ & & $x$ & & & 0.13 & 0 & 0.08 & 0.02 & 0.41 & 0.04 & 0 \\
\hline Fabaceae & Stryphnodendron rotundifolium & $\mathrm{NE}$ & 0.215 & 0.002 & & & $x$ & & & & & 0.02 & 0.56 & 0.08 & 0.68 & 0.37 & 0 & 0.47 \\
\hline Fabaceae & Tachigali aurea & SW & 0.188 & 0.015 & & & $x$ & & & & & 0.32 & 0.8 & 0.08 & 0.55 & 0.35 & 0.52 & 0.8 \\
\hline Fabaceae & Tachigali subvelutina & NE & 0.219 & 0.004 & & & $x$ & & & & & 0.85 & 0.6 & 0.85 & 0.9 & 0.07 & 0.44 & 0 \\
\hline Fabaceae & Vatairea macrocarpa & $\mathrm{CW}$ & 0.205 & 0.005 & & & $x$ & & & & & 0.42 & 0.89 & 0.85 & 0.8 & 0.39 & 0.04 & 0.47 \\
\hline Hypericaceae & Vismia guianensis & $\mathrm{N}$ & 0.455 & 0.001 & $x$ & & $x$ & & & & & 0 & 0.03 & 0.54 & 0.07 & 0 & 0 & 0 \\
\hline Icacinaceae & Emmotum nitens & $\mathrm{NE}$ & 0.213 & 0.003 & & & $x$ & & & & & 0.34 & 0.69 & 0.15 & 0.7 & 0.04 & 0.37 & 0 \\
\hline
\end{tabular}




\begin{tabular}{|c|c|c|c|c|c|c|c|c|c|c|c|c|c|c|c|c|c|c|}
\hline \multirow[t]{2}{*}{ Família } & \multirow[t]{2}{*}{ Espécie } & \multirow[t]{2}{*}{ DB } & \multirow[t]{2}{*}{ ISA } & \multirow[t]{2}{*}{$\mathbf{p}$} & \multicolumn{7}{|c|}{$\begin{array}{l}\text { Biomas de ocorrência } \\
\text { predominante }\end{array}$} & \multicolumn{7}{|c|}{$\begin{array}{l}\text { Frequência das espécies nos Distritos } \\
\text { Biogeográficos }\end{array}$} \\
\hline & & & & & $\mathrm{Am}$ & $\mathrm{Ca}$ & $\mathrm{Ce}$ & $\mathrm{Ch}$ & MA & Pam & Pan & C & CW & $\mathbf{N}$ & NE & $\mathbf{S}$ & SE & sw \\
\hline Lacistemataceae & Lacistema hasslerianum & $\mathrm{S}$ & 0.257 & 0.001 & & & $x$ & & $x$ & & & 0 & 0.02 & 0 & 0 & 0.3 & 0.04 & 0 \\
\hline Lamiaceae & Aegiphila verticillata & $\mathrm{S}$ & 0.162 & 0.012 & & & $x$ & & $x$ & & & 0.49 & 0.21 & 0.15 & 0.23 & 0.63 & 0.59 & 0.13 \\
\hline Lamiaceae & Hyptidendron canum & SE & 0.357 & 0.001 & & & $x$ & & & & & 0.15 & 0.06 & 0 & 0 & 0.02 & 0.52 & 0 \\
\hline Lauraceae & Mezilaurus crassiramea & $\mathrm{CW}$ & 0.182 & 0.004 & $x$ & & $x$ & & & & & 0.02 & 0.25 & 0 & 0 & 0 & 0 & 0.07 \\
\hline Lauraceae & Ocotea corymbosa & $S$ & 0.395 & 0.001 & & & $x$ & & $x$ & & & 0 & 0.01 & 0 & 0 & 0.43 & 0.04 & 0 \\
\hline Lauraceae & Ocotea minarum & SW & 0.353 & 0.001 & & & $x$ & & $x$ & & & 0 & 0.03 & 0 & 0 & 0.02 & 0 & 0.4 \\
\hline Lauraceae & Ocotea pomaderroides & $\mathrm{C}$ & 0.07 & 0.036 & & $x$ & $x$ & & & & & 0.08 & 0.01 & 0 & 0 & 0 & 0 & 0 \\
\hline Lauraceae & Ocotea pulchella & $\mathrm{S}$ & 0.709 & 0.001 & & & $x$ & & $x$ & & & 0.02 & 0 & 0 & 0 & 0.76 & 0.04 & 0 \\
\hline Lauraceae & Ocotea spixiana & $\mathrm{C}$ & 0.097 & 0.019 & & & $x$ & & $x$ & & & 0.11 & 0.02 & 0 & 0 & 0 & 0 & 0 \\
\hline Lecythidaceae & Eschweilera nana & NE & 0.108 & 0.038 & & & $x$ & & & & & 0 & 0.17 & 0 & 0.2 & 0 & 0 & 0 \\
\hline Lythraceae & Lafoensia pacari & $\mathrm{C}$ & 0.163 & 0.015 & & & $x$ & & $x$ & & & 0.57 & 0.36 & 0.31 & 0.13 & 0.26 & 0.33 & 0 \\
\hline Lythraceae & Lafoensia vandelliana & SW & 0.204 & 0.006 & & & $x$ & & $x$ & & & 0.3 & 0.52 & 0.54 & 0.62 & 0.28 & 0.56 & 0.87 \\
\hline Lythraceae & Physocalymma scaberrimum & $\mathrm{CW}$ & 0.256 & 0.002 & $x$ & & $x$ & & & & & 0.04 & 0.31 & 0 & 0.03 & 0 & 0 & 0 \\
\hline Malpighiaceae & Banisteriopsis campestris & $\mathrm{S}$ & 0.061 & 0.021 & & & $x$ & & $x$ & & & 0 & 0 & 0 & 0 & 0.09 & 0.04 & 0 \\
\hline Malpighiaceae & Byrsonima correifolia & $\mathrm{N}$ & 0.231 & 0.001 & & $x$ & $x$ & & & & & 0.02 & 0 & 0.31 & 0.08 & 0 & 0 & 0 \\
\hline Malpighiaceae & Byrsonima intermedia & $\mathrm{S}$ & 0.306 & 0.001 & & & $x$ & & $x$ & & & 0.06 & 0.16 & 0 & 0.1 & 0.65 & 0.22 & 0.2 \\
\hline Malpighiaceae & Byrsonima pachyphylla & NE & 0.209 & 0.003 & & & $x$ & & & & & 0.7 & 0.72 & 0 & 0.78 & 0.13 & 0.41 & 0.2 \\
\hline Malpighiaceae & Byrsonima verbascifolia & SE & 0.188 & 0.01 & & & $x$ & & & & & 0.77 & 0.66 & 0.15 & 0.27 & 0.52 & 0.81 & 0.33 \\
\hline Malpighiaceae & Heteropterys byrsonimifolia & $\mathrm{C}$ & 0.175 & 0.01 & & & $x$ & & $x$ & & & 0.57 & 0.47 & 0 & 0.28 & 0.24 & 0.15 & 0.13 \\
\hline Malvaceae & Eriotheca gracilipes & $\mathrm{S}$ & 0.304 & 0.001 & & & $x$ & & $x$ & & & 0.19 & 0.78 & 0.08 & 0.5 & 0.96 & 0.04 & 0.47 \\
\hline Malvaceae & Eriotheca pubescens & $\mathrm{C}$ & 0.361 & 0.001 & & & $x$ & & & & & 0.75 & 0.25 & 0 & 0.02 & 0.07 & 0.3 & 0.2 \\
\hline Malvaceae & Pseudobombax longiflorum & $\mathrm{CW}$ & 0.263 & 0.001 & & & $x$ & & & & & 0.25 & 0.79 & 0 & 0.1 & 0.3 & 0.52 & 0.4 \\
\hline Malvaceae & Pseudobombax tomentosum & SW & 0.441 & 0.001 & & & $x$ & & & & & 0.11 & 0.38 & 0 & 0.05 & 0 & 0.11 & 0.8 \\
\hline Malvaceae & Sterculia striata & $\mathrm{N}$ & 0.096 & 0.036 & & & $x$ & & & & & 0 & 0.08 & 0.23 & 0.05 & 0 & 0 & 0.2 \\
\hline Marcgraviaceae & Schwartzia adamantium & $\mathrm{C}$ & 0.099 & 0.03 & & & $x$ & & & & & 0.13 & 0.04 & 0 & 0 & 0 & 0 & 0 \\
\hline Melastomataceae & Miconia albicans & $\mathrm{S}$ & 0.252 & 0.001 & & & $x$ & & $x$ & & & 0.42 & 0.46 & 0.15 & 0.35 & 0.91 & 0.81 & 0.2 \\
\hline Melastomataceae & Miconia burchellii & $\mathrm{C}$ & 0.226 & 0.002 & & & $x$ & & & & & 0.26 & 0.04 & 0 & 0 & 0 & 0 & 0 \\
\hline Melastomataceae & Miconia ferruginata & C & 0.463 & 0.001 & & & $x$ & & & & & 0.68 & 0.16 & 0 & 0.12 & 0 & 0.04 & 0 \\
\hline
\end{tabular}




\begin{tabular}{|c|c|c|c|c|c|c|c|c|c|c|c|c|c|c|c|c|c|c|}
\hline \multirow[t]{2}{*}{ Família } & \multirow[t]{2}{*}{ Espécie } & \multirow[t]{2}{*}{ DB } & \multirow[t]{2}{*}{ ISA } & \multirow[t]{2}{*}{ p } & \multicolumn{7}{|c|}{$\begin{array}{l}\text { Biomas de ocorrência } \\
\text { predominante }\end{array}$} & \multicolumn{7}{|c|}{$\begin{array}{l}\text { Frequência das espécies nos Distritos } \\
\text { Biogeográficos }\end{array}$} \\
\hline & & & & & $A m$ & $\mathrm{Ca}$ & $\mathrm{Ce}$ & Ch & MA & Pam & Pan & C & CW & $\mathbf{N}$ & NE & $\mathbf{S}$ & SE & sW \\
\hline Melastomataceae & Miconia leucocarpa & C & 0.486 & 0.001 & & & $x$ & & & & & 0.57 & 0.01 & 0 & 0 & 0.09 & 0 & 0 \\
\hline Melastomataceae & Miconia ligustroides & $S$ & 0.447 & 0.001 & & & $x$ & & $x$ & & & 0.02 & 0.02 & 0 & 0 & 0.57 & 0.11 & 0 \\
\hline Melastomataceae & Miconia paucidens & $\mathrm{S}$ & 0.326 & 0.001 & & & $x$ & & $x$ & & & 0 & 0 & 0 & 0 & 0.33 & 0 & 0 \\
\hline Melastomataceae & Miconia pepericarpa & SE & 0.095 & 0.013 & & & $x$ & & $x$ & & & 0.02 & 0 & 0 & 0 & 0 & 0.11 & 0 \\
\hline Melastomataceae & Miconia rubiginosa & $\mathrm{S}$ & 0.441 & 0.001 & & & $x$ & & $x$ & & & 0.11 & 0.04 & 0 & 0.05 & 0.65 & 0.11 & 0 \\
\hline Melastomataceae & Miconia sellowiana & $S$ & 0.102 & 0.019 & & & $x$ & & $x$ & & & 0.08 & 0 & 0 & 0 & 0.15 & 0 & 0 \\
\hline Melastomataceae & Miconia stenostachya & $\mathrm{S}$ & 0.275 & 0.001 & & & $x$ & & $x$ & & & 0.04 & 0.05 & 0 & 0.08 & 0.41 & 0.04 & 0 \\
\hline Melastomataceae & Mouriri elliptica & NE & 0.264 & 0.001 & & & $x$ & & & & & 0 & 0.54 & 0.15 & 0.6 & 0 & 0 & 0.07 \\
\hline Melastomataceae & Mouriri pusa & NE & 0.276 & 0.001 & & $x$ & $x$ & & & & & 0 & 0.36 & 0.23 & 0.57 & 0 & 0 & 0 \\
\hline Melastomataceae & Tibouchina papyrus & $\mathrm{C}$ & 0.07 & 0.036 & & & $x$ & & & & & 0.08 & 0.01 & 0 & 0 & 0 & 0 & 0 \\
\hline Melastomataceae & Tibouchina stenocarpa & $\mathrm{S}$ & 0.211 & 0.001 & & & $x$ & & $x$ & & & 0.02 & 0.01 & 0 & 0 & 0.26 & 0.04 & 0 \\
\hline Meliaceae & Cabralea canjerana & SE & 0.35 & 0.001 & & & $x$ & & $x$ & & & 0 & 0 & 0 & 0 & 0.02 & 0.37 & 0 \\
\hline Moraceae & Brosimum gaudichaudii & CW & 0.179 & 0.027 & & & $x$ & & & & & 0.4 & 0.77 & 0 & 0.48 & 0.52 & 0.7 & 0.47 \\
\hline Moraceae & Ficus citrifolia & $S$ & 0.196 & 0.001 & $x$ & & $x$ & & $x$ & & & 0 & 0 & 0 & 0 & 0.2 & 0 & 0 \\
\hline Myristicaceae & Virola sebifera & $\mathrm{S}$ & 0.223 & 0.001 & $x$ & & $x$ & & & & & 0.25 & 0.2 & 0 & 0.08 & 0.54 & 0.19 & 0.07 \\
\hline Myrtaceae & Blepharocalyx salicifolius & C & 0.26 & 0.001 & & & $x$ & & $x$ & & & 0.47 & 0.02 & 0 & 0.02 & 0.35 & 0 & 0 \\
\hline Myrtaceae & Campomanesia adamantium & $\mathrm{S}$ & 0.14 & 0.007 & & & $x$ & & $x$ & & & 0.08 & 0.02 & 0 & 0 & 0.24 & 0.07 & 0 \\
\hline Myrtaceae & Eugenia aurata & $\mathrm{S}$ & 0.339 & 0.001 & & & $x$ & & $x$ & & & 0.06 & 0.25 & 0 & 0.02 & 0.59 & 0.11 & 0 \\
\hline Myrtaceae & Eugenia dysenterica & SE & 0.311 & 0.001 & & & $x$ & & & & & 0.38 & 0.54 & 0.31 & 0.43 & 0.11 & 0.93 & 0.07 \\
\hline Myrtaceae & Eugenia hiemalis & $\mathrm{S}$ & 0.239 & 0.001 & & & $x$ & & $x$ & & & 0 & 0 & 0 & 0 & 0.24 & 0 & 0 \\
\hline Myrtaceae & Eugenia pitanga & $\mathrm{S}$ & 0.124 & 0.006 & & & $x$ & & $x$ & & & 0 & 0.01 & 0 & 0 & 0.13 & 0 & 0 \\
\hline Myrtaceae & Eugenia punicifolia & $\mathrm{S}$ & 0.268 & 0.001 & & & $x$ & & $x$ & & & 0.09 & 0.07 & 0.23 & 0 & 0.5 & 0.04 & 0 \\
\hline Myrtaceae & Myrcia albotomentosa & $S$ & 0.274 & 0.001 & & & $x$ & & $x$ & & & 0.02 & 0.13 & 0 & 0.02 & 0.5 & 0.11 & 0.13 \\
\hline Myrtaceae & Myrcia bella & $S$ & 0.336 & 0.001 & & & $x$ & & $\mathrm{x}$ & & & 0.06 & 0.04 & 0 & 0 & 0.41 & 0 & 0 \\
\hline Myrtaceae & Myrcia tomentosa & $\mathrm{S}$ & 0.154 & 0.019 & & & $x$ & & $x$ & & & 0.23 & 0.2 & 0 & 0.02 & 0.5 & 0.41 & 0.27 \\
\hline Myrtaceae & Myrcia uberavensis & $\mathrm{S}$ & 0.132 & 0.006 & & & $x$ & & & & & 0.04 & 0.06 & 0 & 0 & 0.2 & 0 & 0 \\
\hline Myrtaceae & Myrcia variabilis & C & 0.093 & 0.043 & & & $x$ & & & & & 0.19 & 0.03 & 0 & 0 & 0.02 & 0.15 & 0 \\
\hline Myrtaceae & Psidium laruotteanum & C & 0.163 & 0.01 & & & $x$ & & $x$ & & & 0.32 & 0.06 & 0 & 0.1 & 0.11 & 0.04 & 0 \\
\hline
\end{tabular}




\begin{tabular}{|c|c|c|c|c|c|c|c|c|c|c|c|c|c|c|c|c|c|c|}
\hline \multirow[t]{2}{*}{ Família } & \multirow[t]{2}{*}{ Espécie } & \multirow[t]{2}{*}{ DB } & \multirow[t]{2}{*}{ ISA } & \multirow[t]{2}{*}{$\mathbf{p}$} & \multicolumn{7}{|c|}{$\begin{array}{l}\text { Biomas de ocorrência } \\
\text { predominante }\end{array}$} & \multicolumn{7}{|c|}{$\begin{array}{l}\text { Frequência das espécies nos Distritos } \\
\text { Biogeográficos }\end{array}$} \\
\hline & & & & & Am & $\mathrm{Ca}$ & $\mathrm{Ce}$ & $\mathrm{Ch}$ & MA & Pam & Pan & C & CW & $\mathbf{N}$ & NE & $\mathbf{S}$ & SE & sw \\
\hline Myrtaceae & Psidium myrsinites & $\mathrm{N}$ & 0.153 & 0.003 & & & $x$ & & & & & 0.17 & 0.08 & 0.31 & 0.07 & 0 & 0 & 0 \\
\hline Myrtaceae & Psidium myrtoides & $\mathrm{NE}$ & 0.316 & 0.001 & & & $x$ & & $x$ & & & 0.21 & 0.35 & 0.08 & 0.63 & 0 & 0 & 0 \\
\hline Myrtaceae & Psidium pohlianum & $\mathrm{C}$ & 0.082 & 0.026 & & $x$ & $x$ & & & & & 0.11 & 0.04 & 0 & 0 & 0 & 0 & 0 \\
\hline Myrtaceae & Siphoneugena densiflora & C & 0.158 & 0.003 & & & $x$ & & $x$ & & & 0.17 & 0.01 & 0 & 0 & 0 & 0 & 0 \\
\hline Nyctaginaceae & Guapira graciliflora & $\mathrm{C}$ & 0.197 & 0.003 & & & $x$ & & $x$ & & & 0.47 & 0.32 & 0 & 0.08 & 0.15 & 0.04 & 0.07 \\
\hline Nyctaginaceae & Guapira noxia & $\mathrm{C}$ & 0.295 & 0.001 & & & $x$ & & $x$ & & & 0.89 & 0.46 & 0 & 0.02 & 0.83 & 0.48 & 0 \\
\hline Nyctaginaceae & Neea theifera & SE & 0.172 & 0.015 & & & $x$ & & $x$ & & & 0.62 & 0.46 & 0 & 0.32 & 0.28 & 0.63 & 0 \\
\hline Ochnaceae & Ouratea castaneifolia & SE & 0.192 & 0.002 & & & $x$ & & $x$ & & & 0.08 & 0.24 & 0 & 0.03 & 0.11 & 0.41 & 0 \\
\hline Ochnaceae & Ouratea hexasperma & $\mathrm{C}$ & 0.242 & 0.001 & & & $x$ & & & & & 0.89 & 0.77 & 0.46 & 0.77 & 0.02 & 0.15 & 0.2 \\
\hline Ochnaceae & Ouratea spectabilis & $\mathrm{S}$ & 0.481 & 0.001 & & & $x$ & & $x$ & & & 0.09 & 0.3 & 0.08 & 0.15 & 0.93 & 0.26 & 0 \\
\hline Olacaceae & Heisteria ovata & NE & 0.202 & 0.002 & $x$ & & $x$ & & & & & 0.04 & 0.15 & 0 & 0.35 & 0 & 0 & 0.07 \\
\hline Oleaceae & Priogymnanthus hasslerianus & SW & 0.142 & 0.002 & & & $x$ & & & & & 0 & 0.08 & 0 & 0 & 0 & 0 & 0.2 \\
\hline Opiliaceae & Agonandra brasiliensis & $\mathrm{N}$ & 0.173 & 0.026 & & & $x$ & & & & & 0.3 & 0.61 & 0.77 & 0.55 & 0.24 & 0.56 & 0.4 \\
\hline Primulaceae & Myrsine coriacea & $\mathrm{S}$ & 0.217 & 0.002 & & & $x$ & & $x$ & & & 0.25 & 0 & 0 & 0 & 0.46 & 0.26 & 0 \\
\hline Primulaceae & Myrsine guianensis & $\mathrm{C}$ & 0.2 & 0.004 & & & $x$ & & $x$ & & & 0.55 & 0.09 & 0 & 0.02 & 0.2 & 0.19 & 0.47 \\
\hline Primulaceae & Myrsine umbellata & $\mathrm{S}$ & 0.185 & 0.001 & & & $x$ & & $x$ & & & 0.06 & 0.01 & 0 & 0 & 0.33 & 0.19 & 0 \\
\hline Proteaceae & Euplassa inaequalis & $\mathrm{CW}$ & 0.103 & 0.033 & & & $x$ & & & & & 0.04 & 0.15 & 0 & 0.03 & 0 & 0 & 0 \\
\hline Rhamnaceae & Rhamnidium elaeocarpum & SW & 0.464 & 0.001 & & & $x$ & & $x$ & & & 0.04 & 0.31 & 0 & 0 & 0 & 0.07 & 0.73 \\
\hline Rosaceae & Prunus myrtifolia & S & 0.222 & 0.002 & & & $x$ & & $x$ & & & 0.02 & 0 & 0 & 0 & 0.24 & 0 & 0 \\
\hline Rubiaceae & Alibertia edulis & $\mathrm{CW}$ & 0.164 & 0.019 & $x$ & & $x$ & & & & & 0.15 & 0.57 & 0.38 & 0.08 & 0.11 & 0.3 & 0.4 \\
\hline Rubiaceae & Chomelia ribesioides & $C$ & 0.126 & 0.014 & & & $x$ & & & & & 0.21 & 0.08 & 0 & 0 & 0.02 & 0.04 & 0 \\
\hline Rubiaceae & Cordiera elliptica & $\mathrm{NE}$ & 0.101 & 0.029 & & & $x$ & & $x$ & & & 0.08 & 0.04 & 0 & 0.18 & 0 & 0.04 & 0 \\
\hline Rubiaceae & Cordiera macrophylla & $\mathrm{S}$ & 0.121 & 0.02 & & & $x$ & & & & & 0.04 & 0.12 & 0 & 0.02 & 0.22 & 0 & 0 \\
\hline Rubiaceae & Ferdinandusa elliptica & $\mathrm{CW}$ & 0.139 & 0.024 & & & $x$ & & & & & 0.13 & 0.33 & 0 & 0.33 & 0 & 0 & 0 \\
\hline Rubiaceae & Genipa americana & sW & 0.081 & 0.034 & $x$ & & $x$ & & $x$ & & & 0.02 & 0.03 & 0 & 0 & 0 & 0.04 & 0.13 \\
\hline Rubiaceae & Palicourea rigida & $C$ & 0.218 & 0.006 & & & $x$ & & $x$ & & & 0.74 & 0.5 & 0.08 & 0.28 & 0.52 & 0.37 & 0 \\
\hline Rubiaceae & Rudgea viburnoides & SE & 0.156 & 0.016 & & & $x$ & & & & & 0.15 & 0.35 & 0 & 0 & 0.3 & 0.48 & 0.2 \\
\hline Rubiaceae & Tocoyena brasiliensis & $\mathrm{S}$ & 0.083 & 0.031 & & & $x$ & & $x$ & & & 0 & 0 & 0 & 0 & 0.13 & 0.07 & 0 \\
\hline
\end{tabular}




\begin{tabular}{|c|c|c|c|c|c|c|c|c|c|c|c|c|c|c|c|c|c|c|}
\hline \multirow[t]{2}{*}{ Família } & \multirow[t]{2}{*}{ Espécie } & \multirow[t]{2}{*}{ DB } & \multirow[t]{2}{*}{ ISA } & \multirow[t]{2}{*}{$\mathbf{p}$} & \multicolumn{7}{|c|}{$\begin{array}{l}\text { Biomas de ocorrência } \\
\text { predominante }\end{array}$} & \multicolumn{7}{|c|}{$\begin{array}{l}\text { Frequência das espécies nos Distritos } \\
\text { Biogeográficos }\end{array}$} \\
\hline & & & & & $A m$ & $\mathrm{Ca}$ & $\mathrm{Ce}$ & $\mathrm{Ch}$ & MA & Pam & Pan & C & CW & $\mathbf{N}$ & NE & $\mathbf{S}$ & SE & SW \\
\hline Rutaceae & Dictyoloma vandellianum & SE & 0.259 & 0.001 & & & $x$ & & $x$ & & & 0 & 0 & 0 & 0 & 0 & 0.26 & 0 \\
\hline Rutaceae & Zanthoxylum riedelianum & SW & 0.37 & 0.001 & & & $x$ & & $x$ & & & 0.04 & 0.2 & 0 & 0 & 0.02 & 0.11 & 0.6 \\
\hline Sapindaceae & Dilodendron bipinnatum & SW & 0.398 & 0.001 & & & $x$ & & & & & 0 & 0.34 & 0 & 0 & 0 & 0.11 & 0.67 \\
\hline Sapindaceae & Magonia pubescens & SW & 0.231 & 0.001 & & & $x$ & & & & & 0.08 & 0.72 & 0.92 & 0.37 & 0.13 & 0.63 & 0.93 \\
\hline Sapindaceae & Matayba guianensis & CW & 0.191 & 0.006 & $x$ & & $x$ & & $x$ & & & 0.21 & 0.46 & 0 & 0.07 & 0 & 0.04 & 0.33 \\
\hline Sapotaceae & Chrysophyllum marginatum & SW & 0.164 & 0.005 & & & $x$ & & $x$ & & & 0.02 & 0.03 & 0 & 0 & 0.09 & 0.04 & 0.27 \\
\hline Sapotaceae & Pouteria torta & $S$ & 0.193 & 0.006 & $x$ & & $x$ & & $x$ & & & 0.47 & 0.21 & 0 & 0.38 & 0.63 & 0.3 & 0.07 \\
\hline Simaroubaceae & Simarouba versicolor & $\mathrm{N}$ & 0.337 & 0.001 & & & $x$ & & & & & 0.25 & 0.74 & 1 & 0.57 & 0.04 & 0.04 & 0.33 \\
\hline Siparunaceae & Siparuna guianensis & $\mathrm{S}$ & 0.247 & 0.001 & $x$ & & $x$ & & $x$ & & & 0.11 & 0.23 & 0 & 0.2 & 0.59 & 0.26 & 0 \\
\hline Styracaceae & Styrax camporum & SE & 0.436 & 0.001 & & & $x$ & & $x$ & & & 0.13 & 0.03 & 0 & 0 & 0.54 & 0.81 & 0 \\
\hline Styracaceae & Styrax ferrugineus & C & 0.306 & 0.001 & & & $x$ & & $x$ & & & 0.83 & 0.24 & 0 & 0.05 & 0.74 & 0.26 & 0.13 \\
\hline Symplocaceae & Symplocos nitens & SE & 0.335 & 0.001 & & & $x$ & & $\mathrm{x}$ & & & 0.04 & 0.01 & 0 & 0 & 0.04 & 0.41 & 0 \\
\hline Symplocaceae & Symplocos pubescens & $S$ & 0.186 & 0.001 & & & $x$ & & $x$ & & & 0 & 0 & 0 & 0 & 0.22 & 0.04 & 0 \\
\hline Symplocaceae & Symplocos rhamnifolia & C & 0.19 & 0.004 & & $x$ & $x$ & & & & & 0.21 & 0.02 & 0 & 0 & 0 & 0 & 0 \\
\hline Thymelaeaceae & Daphnopsis fasciculata & $\mathrm{S}$ & 0.217 & 0.001 & & & $x$ & & $x$ & & & 0 & 0 & 0 & 0 & 0.22 & 0 & 0 \\
\hline Vochysiaceae & Callisthene molissima & CW & 0.107 & 0.024 & & & $x$ & & & & & 0 & 0.11 & 0 & 0 & 0 & 0 & 0 \\
\hline Vochysiaceae & Qualea cordata & SE & 0.254 & 0.001 & & & $x$ & & $x$ & & & 0 & 0 & 0 & 0.02 & 0.15 & 0.37 & 0 \\
\hline Vochysiaceae & Qualea dichotoma & $\mathrm{S}$ & 0.145 & 0.004 & & & $x$ & & $x$ & & & 0.13 & 0.03 & 0 & 0 & 0.33 & 0.11 & 0.13 \\
\hline Vochysiaceae & Salvertia convallariodora & $\mathrm{N}$ & 0.257 & 0.001 & & & $x$ & & & & & 0.36 & 0.79 & 1 & 0.82 & 0.02 & 0.7 & 0.2 \\
\hline Vochysiaceae & Vochysia cinnamomea & $\mathrm{S}$ & 0.137 & 0.014 & & & $x$ & & & & & 0.09 & 0.14 & 0 & 0.13 & 0.3 & 0 & 0 \\
\hline Vochysiaceae & Vochysia elliptica & $\mathrm{C}$ & 0.327 & 0.001 & & & $x$ & & & & & 0.64 & 0.14 & 0 & 0.07 & 0 & 0.41 & 0 \\
\hline Vochysiaceae & Vochysia gardneri & NE & 0.392 & 0.001 & & & $x$ & & & & & 0 & 0.09 & 0.08 & 0.52 & 0 & 0 & 0 \\
\hline Vochysiaceae & Vochysia haenkeana & CW & 0.107 & 0.027 & $x$ & & $x$ & & & & & 0.02 & 0.21 & 0.08 & 0.1 & 0 & 0 & 0 \\
\hline Vochysiaceae & Vochysia rufa & CW & 0.163 & 0.025 & & & $x$ & & & & & 0.55 & 0.6 & 0 & 0.28 & 0.04 & 0.56 & 0.2 \\
\hline Vochysiaceae & Vochysia thyrsoidea & $\mathrm{C}$ & 0.463 & 0.001 & & & $x$ & & $\mathrm{x}$ & & & 0.68 & 0.07 & 0 & 0.1 & 0 & 0.15 & 0 \\
\hline Vochysiaceae & Vochysia tucanorum & $\mathrm{S}$ & 0.36 & 0.001 & & & $x$ & & $x$ & & & 0.17 & 0.04 & 0.08 & 0.1 & 0.7 & 0.26 & 0 \\
\hline
\end{tabular}




\section{CAPÍTULO 3. CONTRIBUIÇÃO DE FATORES CLIMÁTICOS E EDÁFICOS PARA A DISTRIBUIÇÃO DAS ESPÉCIES LENHOSAS DO CERRADO}

\section{RESUMO}

Os filtros ambientais atuam na distribuição das espécies, sendo os gradientes climáticos e edáficos os mais importantes para a distribuição das plantas. As condições ambientais e os recursos atuam como uma força seletiva dos traços das espécies, selecionando espécies com requerimentos semelhantes. Essas características podem ter origem histórica, quando provem de um ancestral comum que partilhava a mesma característica, ou ecológica, quando por pressão externa, espécies de diferentes linhagens convergem em traços semelhantes. No Cerrado muita ênfase tem sido dada ao papel do solo na diferenciação das comunidades, mas não se sabe ao certo sua verdadeira importância. Assim, o objetivo desse estudo é comparar a importância de variáveis climáticas e edáficas na composição das comunidades arbóreas do cerrado, e verificar se há conservatismo de nicho em relação às variáveis mais importantes. Para isso realizei uma análise de partição de variância em três escalas usando 87 comunidades arbóreas de cerrado sentido amplo, 11 variáveis climáticas e 11 variáveis edáficas. Testamos individualmente a importância dessas variáveis para a ocorrência de cada uma das 303 espécies analisadas. Testamso o conservatismo de nicho dessas características. A matriz climática foi mais importante para a determinação da comunidade nas escalas mais amplas, no entanto na menor escala e nas análises específicas o solo teve maior contribuição. Dentre as variáveis mais importantes, apenas preferência por soma de bases apresentou conservatismo de nicho, o que já foi demonstrado em outros ecossistemas. O clima atua como filtro primário, determinando o pool regional de espécies, e o solo atua em uma escala mais fina, aumentando a heterogeneidade ambiental e a diversidade filogenética do cerrado. Estudos sobre potencial de dispersão das árvores do cerrado são importantes no presente cenário de mudanças climáticas aceleradas. 
A composição de comunidades tem sido tópico de grande importância na ecologia. Os princípios que regem a composição das comunidades são derivados da teoria neutra (Hubbell 2001) e da teoria de nicho (Grinnell 1917; Hutchinson 1957). A teoria neutra postula que as comunidades ecológicas são abertas, continuamente em mudança e não existe um equilíbrio, pois a presença, ausência e abundância relativa das espécies são governadas por especiações e dispersões aleatórias, deriva ecológica e extinção (Hubbell 2001). Já a teoria de nicho tem uma visão determinística, onde a ocorrência das espécies é condicionada por fatores ambientais aos quais as espécies estão aptas a tolerar. A teoria de nicho foi formulada inicialmente por Grinnell (1917), como um atributo do habitat em que a espécie vive. Essa teoria foi modificada por Hutchinson (1957), segundo o qual o nicho é uma característica de cada espécie, determinado por um hipervolume multidimensional, composto por todos os requisitos fisiológicos e biológicos da espécie, constituindo o seu nicho fundamental.

Os processos que determinam a composição das comunidades de plantas (e. g. germinação de sementes, estabelecimento de plântulas, tolerâncias fisiológicas, efeitos demográficos decorrentes de distúrbios, competição e outras interações biológicas) são significativamente associados às condições ambientais (Ackerly 2003). O ambiente atua como filtro na medida em que seleciona as espécies com condições mínimas para ocorrerem em uma localidade. Nesse sentido, o clima e o solo têm sido apontados como os maiores preditores da distribuição das plantas (Furley \& Ratter 1988; Punyasena et al. 2008).

As plantas são bastante afetadas pelo clima (Punyasena et al. 2008), o qual determina sua ocorrência em diversas escalas (Clements \& Shelford 1939; Prentice et al. 1992; Schimel et al. 1997; Skarpe 1996). Importantes processos fisiológicos relacionados à constituição estrutural e ao metabolismo são dependentes da temperatura, umidade (condições ambientais), água e radiação (recurso) (Gurevitch et al. 2009). A formação do solo é dependente do regime climático e de intemperismo sendo, portanto, estreitamente relacionado ao clima (Raij 1981). No entanto, as variações edáficas decorrentes de outras causalidades, como material de origem ou relevo, variam em escalas menores na paisagem (Pearson \& Dawson 2003). Textura, acidez e disponibilidade de nutrientes são as principais características edáficas que determinam o sucesso do estabelecimento das plantas (Gurevitch et al. 2009; Raij 1981). 
Diferentes espécies lidam com os recursos e com as condições ambientais de diferentes maneiras (Skarpe 1996). Por isso, comunidades distintas são observadas ao longo de gradientes climáticos e edáficos (Gurevitch et al. 2009; Stephenson 1990). No entanto, quando tolerâncias fisiológicas são compartilhadas por diferentes espécies ao longo de uma mesma linhagem evolutiva, os requerimentos ambientais são semelhantes, caracterizando o conservatismo de nicho (Cavender-Bares et al. 2009).

Examinar o conservatismo de nicho associado aos gradientes ambientais auxilia na interpretação dos processos que geram a composição das comunidades, aspecto de extrema relevância frente às mudanças globais experimentadas em diversas escalas (Cavender-Bares et al. 2009). No entanto, devido à complexidade dos padrões que agem de maneira simultânea sobre diferentes comunidades e em diferentes escalas, determinar o papel dos filtros ambientais, dos eventos históricos e da limitação de dispersão das espécies na composição de comunidades não é uma tarefa trivial (Leibold et al. 2010).

\section{Vegetação do Cerrado e filtros ambientais}

O bioma Cerrado (sensu Clements \& Shelford 1939) é a maior savana neotropical, com cerca de dois milhões de quilômetros quadrados. As formações savânicas, predominantes nessa paisagem, são intercaladas por florestas e campos (Eiten 1972; Ribeiro \& Walter 1998, 2008). Devido ao alto endemismo de plantas e grande alteração ambiental, o Cerrado é considerado um hotspot de biodiversidade (Myers et al. 2000).

O clima e o solo estão entre os principais fatores abióticos que atuam como filtros ambientais para as comunidades arbóreas do Cerrado (Batalha et al. 2011; Castro 1994; Durigan \& Ratter 2006; Furley \& Ratter 1988; Torres et al. 1997). As condições climáticas variam consideravelmente dentro do bioma (Furley \& Ratter 1988), com temperaturas médias oscilando entre 19 e 25 ㄷ, e a precipitação entre 650 e 2800 mm, concentrada nos meses mais quentes (Camargo 1963). Os solos do Cerrado são antigos, profundos, bem drenados, com alto grau de intemperismo, que promove a lixiviação da sílica, preservando sexquióxidos de ferro, alumínio e manganês (Ranzani 1963). Diversas classes de solo são observadas no Cerrado, com predominância de latossolos distróficos com pequenas quantidades de bases trocáveis e alta saturação de Al (Arens 1963; Haridasan 2000; Ranzani 1963), no entanto manchas de solos 
férteis são comuns em todo o bioma (Felfili et al. 1994; Furley \& Ratter 1988; Furley 2006; Moreno et al. 2008).

Estudos palinológicos sugerem que ocorreram grandes modificações na vegetação do Cerrado provocadas por flutuações climáticas durante o Quaternário (Barberi et al. 2000; Ferraz-Vicentini \& Salgado-Labouriau 1996; Ledru 1993; Ledru et al. 1998; Salgado-Labouriau 1997). A reconstrução climática desses períodos mostram flutuações consistentes com essas alterações na vegetação (Werneck et al. 2012). No presente, temperatura mínima anual, sazonalidade da temperatura e precipitação anual são condições climáticas limiares para a ocorrência de comunidades vegetais distintas (Castro 1994; Cap. 2).

As plantas do Cerrado desenvolveram diferentes estratégias para lidar com a estacionalidade climática. Algumas árvores possuem sistemas radiculares desenvolvidos, que atingem camadas constantemente úmidas, o que as permite continuar transpirando livremente durante a estação seca, mesmo nas horas mais quentes do dia (Ferri \& Coutinho 1958). O fechamento estomático noturno reduz a transpiração, estabelecendo o balanço hídrico entre o solo e a planta (Franco 2002). Algumas plantas do cerrado possuem raízes pouco desenvolvidas (Franco 2002), e possivelmente se beneficiam pelo fenômeno conhecido como ascensão hidráulica, pelo qual os horizontes superiores aumentam o potencial hídrico (Gurevitch et al. 2009). Um complexo sistema de deciduidade é observado nas comunidades do cerrado como estratégia para evitar a perda de água (Bucci et al. 2004; Franco 2002). Em relação ao modo reprodutivo, cerca de um terço das espécies possuem estruturas subterrâneas de propagação vegetativa (Borges 2000), já que o estabelecimento de plântulas a partir de sementes é extremamente dificultado pela sazonalidade climática (Franco 2002). Essas e outras adaptações são importantes para o estabelecimento e sobrevivência das plantas em decorrência das condições climáticas.

O gradiente edáfico de fertilidade é acompanhado por um gradiente de densidade arbórea, onde as fitofisionomias mais densas estão sobre solos mais férteis (Goodland \& Pollard 1973). O solo influencia a área basal total em comunidades do cerrado, o que não ocorre nas demais savanas mundiais (Lehmann et al. 2014). No entanto, há a ocorrência tanto de cerrado típico quanto de cerradão em diferentes extremos no gradiente de fertilidade. As comunidades do cerrado se diferenciam devido à textura do solo, fertilidade, concentração de Al e rochosidade (e. g. Amorim \& Batalha 2007; Abreu et al. 2012; Silva \& Batalha 2008; 
1451 Ruggiero et al. 2002; Marimon-Junior et al. 2005; Neri et al. 2012), já que diferentes 1452 propriedades do solo selecionam plantas com diferentes características e requerimentos 1453 (Batalha et al. 2011).

O alumínio (Al) presente no solo em grandes quantidades é tóxico para a maioria das plantas. No entanto, diversas espécies nativas são adaptadas a essa condição, e muitas dessas são acumuladoras, algumas vezes obrigatórias desse elemento (Haridasan 1982, 2008). Essa característica foi observada para 52 famílias de Angiospermas, mas é predominante nas espécies da ordem Myrtales, sendo uma característica simplesiomórfica desse grupo (Jansen 2002). No cerrado, as espécies acumuladoras de Al são predominantemente das famílias Vochysiaceae e Melastomataceae (Andrade et al. 2011; Haridasan 1982), ambas da ordem Myrtales. Essas espécies apresentam altos valores de importância nas comunidades do cerrado, sugerindo que essa adaptação promova alguma vantagem competitiva sobre as demais espécies (Haridasan 2008). Apesar de bem menos relatado, o Manganês também está presente em alguns solos do cerrado, e também apresenta toxidez a algumas espécies de plantas (Haridasan 2008).

Diferenças na fertilidade do solo aparecem como os principais determinantes das comunidades arbóreas no cerrado, condicionando a ocorrência da maioria das espécies (Bueno; Neves; et al. 2013; Felfili et al. 1994; Franco 2002; Furley \& Ratter 1988; Haridasan 2000), sendo algumas muito características de cada uma dessas condições (Ratter et al. 1973a). O predomínio de algumas famílias foi observado em solos férteis (Torres et al. 1997). No entanto, espécies de solos mesotróficos são observadas eventualmente em solos distróficos, sugerindo uma tolerância das espécies aos solos com menor fertilidade (Bueno; Rafael; et al. 2013). As adaptações das plantas relacionadas à absorção de nutrientes são de difícil mensuração, pois dependem do entendimento da estrutura radicular e da complexa fisiologia envolvida nesse processo (Gurevitch et al. 2009).

Tanto as variações nas condições climáticas quanto edáficas têm potencial de produzir diferentes comunidades, e extremidades desses gradientes podem ter selecionado certas linhagens de plantas. Estudos abordando ambiente e filogenia são importantes na elucidação da importância dos filtros ambientais e da história aplicados ao Cerrado (Batalha et al. 2011). Nesse sentido, poucos estudos inferem as relações entre comunidades, clima e solos (Toledo et al. 2012). Dessa forma, o presente estudo tem como objetivo avaliar o papel do clima e dos 
solos na composição das comunidades de plantas do cerrado. Para isso, testei as seguintes hipóteses: (1) a composição das comunidades é igualmente determinada por variáveis climáticas e edáficas; (2) variáveis climáticas e edáficas atuam como filtros ambientais independentemente da escala de análise; (3) existe conservatismo de nicho relacionado às variáveis climáticas e edáficas para as espécies de plantas arbóreas do Cerrado.

\section{MÉTODOS}

\section{Área de estudo}

O bioma Cerrado, sensu Clements \& Shelford (1939) é dividido em três formações, baseado na estrutura da vegetação: formações campestres, savânicas e florestais 0 cerrado sentido amplo engloba o cerrado sentido restrito e o cerradão, pois essa última, apesar de pertencer à formação florestal, floristicamente se assemelha ao cerrado sentido restrito (Ribeiro \& Walter 2008). Dessa forma, nossas análises se concentraram em localidades de cerrado sentido amplo (que será designado apenas como cerrado) por toda a extensão do Cerrado, incluindo encraves nos outros biomas (Figura 1).

Base de dados

Reunimos estudos florísticos e fitossociológicos incluindo análises da composição dos solos. Para isso, partimos das pesquisas realizadas na expedição anglo-brasileira Xavantina Cachimbo e pelo projeto Conservação e Manejo da Biodiversidade do Bioma Cerrado, uma colaboração entre a Empresa Brasileira de Pesquisas Agropecuárias (EMBRAPA), Universidade de Brasília (UnB) e Royal Botanic Garden Edinburgh (RBGE) (Ratter et al. 1973b). Além desses, foram incluídos estudos publicados em periódicos especializados e quatro novas localidades em lacunas importantes de estudos no Cerrado (Capítulo 1 da tese), onde realizamos inventários sistemáticos da vegetação de acordo com o manual de parcelas permanentes para o Cerrado e Pantanal (Felfili et al. 2005). No total, 87 localidades foram compiladas para as quais haviam listas florísticas e análises de solos. A taxonomia e nomenclatura foram atualizadas de acordo com o APG III (2009), e a Lista de Espécies da Flora do Brasil (JBRJ 2014).

Usamos 11 variáveis bioclimáticas (Tabela 1) baseadas em temperatura, precipitação, radiação e umidade (Kriticos et al. 2012). As variáveis bioclimáticas possuem formato matricial e com resolução de 1 km, e são o resultado da interpolação da média dos dados climáticos 
mensais de estações climáticas no período de 30 (1960-1990) a 50 anos (1950-2000), de acordo com a sua disponibilidade (Hijmans et al. 2004). Essas variáveis são frequentemente usadas para a previsão de ocorrência de espécies de diversos grupos taxonômicos, sendo que a flora é significantemente afetada pelo clima (Punyasena et al. 2008).

As variáveis edáficas usadas são baseadas em textura, fertilidade, acidez e toxidez do solo (Tabela 1). Soma de Bases (SB expressa em $\mathrm{cmol}_{\mathrm{c}} / \mathrm{dm}^{3}$ ) indica o número de cargas negativas dos coloides do solo (ou fração argila) ocupadas pelos cátions básicos trocáveis, sendo um parâmetro de alcalinidade do solo. A capacidade de troca catiônica efetiva (CTC = SB + Al expressa em $\mathrm{cmol}_{\mathrm{c}} / \mathrm{dm}^{3}$ ) é composta pelos cátions básicos ( $\mathrm{K}, \mathrm{Ca}$ e $\mathrm{Mg}$ ) e ácidos (Al), e reflete a capacidade do solo em reter cátions no seu pH natural. A saturação por alumínio (m\%) por potássio $(\mathrm{k} \%)$, cálcio (Ca\%) e magnésio $(\mathrm{Mg} \%)$ são as proporções da CTC ocupada por cada um desses elementos. O Al é particularmente importante pela toxidade conferida a esse cátion. A proporção de areia está relacionada à capacidade de retenção de água e nutrientes, sendo que em solos mais arenosos sua retenção é menor, já que a maioria das partículas do solo é de tamanho maior do que $0,2 \mathrm{~mm}$. A acidez do solo $(\mathrm{pH})$ determina a maneira com que as substâncias da solução do solo serão liberadas. (Raij 1981). Alguns estudos consultados traziam informações incompletas. Esses dados são referentes a textura, que não foi analisada em alguns estudos, e informações pontuais da expedição Xavantina-Cachimbo que, pelo desgaste das anotações, foi perdida. Completamos essas informações usando imputação múltipla usando Especificação Totalmente Subordinada (Fully Conditional Specification - FCS) de dados no pacote mice, com comando de mesmo.

\section{Análises}

Para testar a importância das variáveis ambientais na composição das comunidades e quantificar a importância de cada grupo de variáveis (climáticas e edáficas), realizamos uma análise de partição de variância (Borcard et al. 1992) usando o pacote vegan (Oksanen et al. 2014), a qual realiza uma série de ordenações canônicas de cada matriz explicativa em relação à matriz resposta, de maneira combinada e individual. Essa análise foi precedida por uma seleção progressiva de variáveis do pacote packfor (Dray et al. 2011), que seleciona as variáveis explicativas significativas na presença das demais variáveis, por permutação do modelo reduzido, seguindo uma ordem de importância das variáveis (Blanchet et al. 2008). Esse procedimento é realizado para cada conjunto de variáveis climáticas, edáficas e espaciais 
separadamente. Testamos a significância do modelo e dos seus termos por 1000 aleatorizações de Monte Carlo no pacote vegan (Oksanen et al. 2014). Para testar a autocorrelação espacial dos modelos das três escalas (ver abaixo), testamos a significância da explicação do resíduo por uma matriz de coordenada principais da vizinhança (sigla em inglês PCNM), construída usando a matriz de distância euclidiana das coordenadas originais de cada inventário. Em casos de explicação significativa, as variáveis espaciais devem ser usadas como co-variável nos modelos.

Para identificar a contribuição das variáveis climáticas e edáficas em diferentes escalas, realizamos a partição de variância para dois subconjuntos das localidades (Figura 1), sendo a primeira todas as amostras do bioma, a segunda apenas as localidades do Distrito Biogeográfico Nordeste - DBNE (Capítulo 2 dessa tese) e a terceira escala é composta pelas localidades do estado do Tocantins localizadas dentro do DBNE.

Para avaliar a importância das variáveis ambientais na ocorrência das espécies na escala mais ampla, selecionamos as variáveis edáficas e climáticas separadamente pelo critério de informação de Akaike. Essa seleção de modelos foi realizada de maneira progressiva, pela adição de variáveis ao modelo nulo, em uma análise de correspondência canônica (CCA) no pacote vegan (Oksanen et al. 2014). Variáveis selecionadas pelo modelo com alta correlação entre si (maior que 0,7 ) foram excluídas.

Com o intuito de identificar as variáveis ambientais mais importantes para as espécies de maneira individual, realizamos uma regressão logística para cada espécie usando os dados de presença e ausência das espécies e os valores das variáveis climáticas e edáficas por localidade. A significância das variáveis foi testada por 1000 aleatorizações de Monte Carlo. Para essa análise usamos apenas as variáveis selecionadas por critério de Akaike.

Para visualizar a direção da contribuição de cada variável para as espécies, realizamos uma análise de componentes principais usando valores de associação entre cada espécie e cada variável ambiental. Esses valores de associação foram calculados pela média dos valores de cada variável para os sítios em que as espécies ocorreram (Material Suplementar 1).

Construímos uma hipótese filogenética para as 303 espécies registradas, usando filogenias publicadas em estudos anteriores (APGIII et al. 2009; Duarte et al. 2011; Pennington 
2003) e com comprimentos de ramos determinados pelo método de Grafen (1989), usando $\rho=$ 1.

Consideramos os valores da associação das espécies com os gradientes ambientais como parte do nicho das espécies (Prinzing et al. 2001), e testamos o conservatismo de nicho dessas variáveis pelo teste de sinal filogenético no pacote phytools (Revell 2012), usando a estatística $\mathrm{K}$ de Blomberg e $\lambda$ de Pagel. As duas abordagens são complementares, e são encorajadas especialmente em filogenias incipientes (Diniz-Filho et al. 2010). Nos casos de sinal filogenético, os valores de associação entre as espécies e as variáveis foram agrupados em seis classes de preferência de hábitat (mais baixo=1; mais alto=6), as quais foram usadas para reconstruir o estado ancestral, utilizando parcimônia no aplicativo Mesquite (Maddison \& Maddison 2014).

\section{RESULTADOS}

Registramos 303 espécies, pertencentes a 58 famílias e 21 ordens (Material Suplementar 1). As famílias com maior número de espécies foram Fabaceae $(n=58)$, Myrtaceae $(n=19)$, Rubiaceae $(n=16)$ e Vochysiaceae $(n=16)$. Dentre as ordens, Fabales $(n=58)$, Myrtales $(n=57)$ e Malpighiales $(n=49)$ foram as mais numerosas.

\section{Partição de Variância}

Do total de 22 variáveis, oito variáveis bioclimáticas e seis variáveis edáficas resultaram da seleção progressiva para a primeira escala (Tabela 2). Na partição de variância, o poder de explicação de todas as variáveis em conjunto foi de $22 \%$. A maior parte foi explicada pelas duas matrizes em conjunto e nas frações individuais, o clima foi mais importante seguida do solo (Figura 2; Tabela 3). Tanto o modelo $\left(F_{14,72}=2,72 ; p=0,001\right)$ quanto as frações individuais de clima $\left(F_{8,72}=3,43 ; p=0,001\right)$ e solo $\left(F_{6,72}=1,76 ; p=0,001\right)$ foram significativos a $5 \%$ de probabilidade. A explicação dos resíduos pelos $P C N M s$ não foi significativa $\left(F_{11,75}=0,95\right.$; $p=0,806)$.

Na segunda escala (Distrito Biogeográfico Nordeste), quatro variáveis climáticas e três edáficas foram selecionadas (Tabela 2). Vinte e nove por cento da variação dos dados foram significativamente explicados pelas três matrizes, dos quais $23 \%$ foram explicados pela 
1598 intersecção das matrizes. Das frações individuais o clima foi mais importante (Figura 2; Tabela 3). A explicação dos resíduos pelos PCNMs não foi significativa nesta escala $\left(F_{6,27}=0,57 ; p=1\right)$.

1600

1601

1602

1603

1604

1605

Na terceira escala (localidades do estado do Tocantins) apenas uma variável climática e uma edáfica compuseram a partição de variância (Tabela 2), explicando 18\% da composição das comunidades (Figura 2; Tabela 3). Nessa escala, o solo foi mais importante e a variável climática não foi significativa no modelo. A explicação dos resíduos pelos PCNMs não foi significativa $\left(F_{2,10}=1,01 ; p=0,459\right)$.

\section{Filtros ambientais e sua associação com as espécies}

Dentre as 11 variáveis edáficas, o modelo com o menor valor de AIC é composto pelas variáveis Al, pH e SB (Tabela 4). Das 11 variáveis climáticas, o modelo selecionado pelo menor valor de AIC foram B05, B30, B23, B12, B20, B04 e B15 (Tabela 4). Após retirar as variáveis mais correlacionadas, mantive as variáveis B05, B30 e B23.

Nas regressões logísticas, das 303 espécies analisadas, 175 espécies foram significativamente influenciadas por pelo menos uma das variáveis, sendo 39 influenciadas apenas por uma ou mais variáveis climáticas e 52 por variáveis edáficas (Material Suplementar 2). Espécies influenciadas tanto por clima quanto por solo somam 84. As variáveis climáticas com maior número de associações significativas foram temperatura máxima (92), seguido de sazonalidade da radiação (70) e menor índice de umidade anual (55). Dentre as variáveis edáficas, o maior número de associações foi observado para soma de bases (95) e saturação por alumínio (95) e pH (77). Das 239 espécies com associação significativa, 148 apresentaram associação com mais de uma variável.

O primeiro eixo da PCA explicou $46 \%$ da variação dos dados e o segundo explicou $21 \%$. As variáveis mais importantes no primeiro eixo foram sazonalidade da radiação (Bio23) e saturação por Alumínio no eixo negativo e menor índice de umidade semanal (Bio30) no eixo positivo. Soma de bases foi a variável negativa mais importante no eixo dois. Os vetores da soma de bases e do pH têm a mesma direção no segundo eixo, no entanto há um pequeno grupo de espécies mais correlacionado ao $\mathrm{pH}$. A maior parte das espécies apresenta forte associação com soma de bases, que é a variável com maior importância no eixo 2 (Figura 3; Material Suplementar 2). Temperatura máxima da semana mais quente e menor índice de 
umidade exercem a mesma influência na distribuição das espécies. As variáveis sazonalidade da radiação e saturação por Alumínio têm poder explicativo semelhante no primeiro eixo.

\section{Conservatismo de nicho}

Dentre as seis variáveis edáficas e climáticas selecionadas, apenas soma de bases é um gradiente ambiental cuja associação das espécies está conservada filogeneticamente (Tabela 5). A reconstrução do estado ancestral mostrou padrões divergentes entre diferentes ordens (Figura 4). O estado ancestral do clado que engloba as ordens Magnoliales e Laurales (Figura 5), mostrou uma adaptação recente à preferência por solos pobres, já que o estado ancestral mapeado é de níveis intermediários de SB (classe 4) e a maioria das espécies apresentou preferência por níveis mais baixos de SB. As duas espécies representantes da ordem Proteales tiveram preferência por solos com maior quantidade de SB (classe 5), assim como as espécies da ordem Rosales.

Na ordem Fabales, o estado ancestral predominante foi a preferência por solos com quantidades intermediárias/altas de SB (Figura 5). As espécies do gênero Bauhinia são mais basais e tiveram preferência por solos mais férteis, assim como o grupo dos gêneros Copaifera, Peltogyne e Hymenaea, sendo que nesse último são observadas mudanças do estado do caractere. O mesmo é observado para dois grupos derivados, o primeiro composto por espécies dos gêneros Parkia, Stryphnodendron, Mimosa, Enterolobium e Albizia, e o segundo pelos gêneros Andira, Vatairea, Bowdichia e Leptolobium.

As ordens Oxalidades, Celastrales, Malpighales e Asterales (Figura 6) apresentaram preferência por solos com menor quantidade de SB, com algumas reversões do estado de caractere. O clado composto pelas ordens Malvales e Sapindales (Figura 6) apresentou como estado ancestral a preferência por solos férteis, tendo grupos e espécies isoladas com alteração de estado de caractere. Em relação às Myrtales (Figura 6), o estado ancestral mostrou preferência por concentração intermediária/alta de SB, porém diversas espécies apresentaram associação aos solos com menor quantidade de SB, como as espécies dos gêneros Psidium, Vochysia e Miconia.

$\mathrm{Na}$ ordem Ericales (Figura 7), houve alternância no estado de caractere entre espécies do mesmo gênero, como Pouteria, Diospyros e Symplocos, o que também é observado nas 
ordens Boraginales e Apiales. O estado ancestral da ordem Gentinales foi caracterizado por preferência por menores quantidades de SB, no entanto, há uma reversão desse estado na família Rubiaceae e no gênero Aspidosperma, onde a maioria das espécies apresentaram preferência por altos valores de SB (Figura 7). A ordem Lamiales apresentaram estado ancestral de preferência por menores quantidades de SB, no entanto a família Bignoniaceae é predominantemente de solos com alta quantidade de bases (Figura 7).

\section{DISCUSSÃO}

\section{Filtros ambientais}

Nesse estudo avaliamos a importância das variáveis edáficas e climáticas para a composição das comunidades. De acordo com a partição da variância, as variáveis climáticas compõem o filtro ambiental mais importante em maiores escalas para determinar a composição das comunidades do cerrado. Apenas em escala local o solo passou a ser mais importante para determinar as comunidades.

Muitos estudos corroboram diferenças nas comunidades em decorrência de gradientes climáticos, nos diversos ecossistemas (e. g. Mckenzie et al. 2003; Kubota et al. 2014; Greve et al. 2011; Bongers et al. 1999). As diferentes classes de cobertura do solo no continente africano são mais bem diferenciadas pelo clima do que por influência antropogênica, sendo precipitação anual a variável climática mais importante (Greve et al. 2011). Em florestas temperadas no arquipélago do Japão, a diversidade beta é explicada pela temperatura mínima experimentada no inverno (Kubota et al. 2014). Nas florestas temperadas das Rocky Montains, as coníferas respondem significativamente tanto a gradientes de umidade quanto de temperatura (Mckenzie et al. 2003). Nas florestas tropicais no oeste da África, as espécies respondem de diferentes maneiras ao clima, sendo precipitação média anual, comprimento do período seco e déficit hídrico cumulativo as variáveis mais importantes (Bongers et al. 1999). Em outro estudo de maior escala na mesma região, observou-se que a precipitação anual responde pela probabilidade de ocorrência de 73\% das espécies (Holmgren \& Poorter 2007). Esses resultados sugerem que em diversos ecossistemas, o pool regional de espécies é formado pelo clima, provocando migrações, extinções e especiações (Cavender-Bares et al. 2009). 
No Cerrado, quando avaliamos as comunidades arbóreas, observamos que as variáveis mais importantes na escala mais ampla são temperatura máxima anual e precipitação durante a estação seca. Na savana africana, precipitação é a variável mais importante para diferenciar as classes de vegetação (Greve et al. 2011). A precipitação efetiva no Cerrado é a maior dentre as savanas, e ao contrário do observado para as savanas da África ou Austrália, essa variável não explica diferenças na estrutura da vegetação (Lehmann et al. 2014).

Apesar do papel secundário do solo na composição das comunidades do cerrado, acreditamos que esse desempenhe papel fundamental na manutenção desse ecossistema, pois o clima observado no bioma difere em muito do clima observado para as demais savanas (Lehmann et al. 2014). Outras características ambientais de variação local influenciam em menor escala a composição das comunidades sob condições climáticas homogêneas (Leibold et al. 2010; Toledo et al. 2012). Nossos resultados mostraram que no cerrado o solo é mais importante do que o clima em escalas menores, sugerindo que, localmente, esse seja um filtro ambiental determinante para a ocorrência de algumas espécies. O mesmo padrão foi observado na floresta amazônica na Bolívia, onde uma menor proporção das espécies foi associada ao solo do que ao clima (Toledo et al. 2012).

Outros estudos com enfoque apenas nos fatores edáficos e vegetação mostraram associações significativas, em diversas escalas. Na floresta tropical da Costa Rica, $25 \%$ das espécies estudadas tiveram associação significativa às classes de solos (Clark et al. 1999). Em pequena escala eles observaram que o solo é mais importante para determinar a ocorrência das espécies do que o espaço, sugerindo que a teoria de nicho se aplica melhor nessa situação do que a teoria neutra (John et al. 2007). Em uma floresta tropical de Bornéu, observou-se que o gradiente de fertilidade do solo determina a composição das comunidades (Potts et al. 2002).

Parte da explicação da composição das comunidades é ainda dada pelos dois fatores em conjunto. Isso é esperado, já que de modo geral o clima é um dos fatores mais importantes na formação dos solos (Raij 1981). Além disso, certas características climáticas interagem com o solo, promovendo condições únicas. Um exemplo é a umidade do solo, que depende tanto da textura do solo quanto do regime hídrico, e pode ser uma condição determinante para a ocorrência de algumas espécies. Em uma floresta tropical do Istmo do Panamá, a dessecação do solo foi apontada como moldadora da distribuição de diversas espécies, tanto em escala 
regional como local, sendo apontada como um fator seletivo dessas comunidades (Engelbrecht et al. 2007).

Os filtros ambientais em menor escala são importantes para aumentar a diversidade regional (Leibold et al. 2010). A riqueza de espécies do cerrado, por exemplo, do provém principalmente da heterogeneidade de habitats (Felfili et al. 1994), que aumentam a amplitude da similaridade entre as comunidades mais próximas. Não apenas diferentes classes de solos, mas também o relevo, frequência de fogo e interações bióticas atuam na diversificação das comunidades arbóreas (Silva \& Batalha 2009).

\section{Associação entre espécies e variáveis ambientais}

As espécies respondem de diferentes maneiras às variáveis ambientais, por isso os filtros são seletivos. Dessa forma avaliamos a importância das variáveis ambientais para cada espécie. No nível de espécie, o gradiente edáfico foi mais importante, determinando a ocorrência de uma quantidade de espécies maior do que o clima (Material Suplementar 2), como sugerido por Ratter \& Dargie (1992). No Cerrado, como o clima determina o pool regional de espécies, regiões com maior diversificação de solos e outras variáveis locais, como relevo, litologia entre outros devem ter maior diversidade de organismos. Esses padrões devem ser testados em novos estudos.

A diferença nos resultados entre o nível de comunidade e específico sugere que, apesar de existir tendência geral do gradiente climático explicar a composição das comunidades, as variáveis edáficas têm maior poder determinístico. Poucas espécies apresentam forte associação às variáveis climáticas, pois as espécies que apresentam altos scores absolutos são influenciadas pelo eixo 2, que é predominantemente guiado por variáveis edáficas, tais como soma de bases e pH. Apesar de não serem correlacionadas, soma de bases e pH têm um papel muito semelhante no solo em linhas gerais, pois o $\mathrm{pH}$, dentre outras coisas, é dependente da proporção de bases que ocupa a argila do solo. Altos valores de soma de bases e de $\mathrm{pH}$ permitem que os nutrientes sejam liberados com mais facilidade, representando a fertilidade do solo. No entanto soma de bases é um parâmetro mais eficiente para determinar a ocorrência das espécies que têm exigência de solos férteis. Os solos tropicais são ácidos devido à alta lixiviação das bases trocáveis em decorrência da alta precipitação, permanecendo os 
1743 cátions ácidos (Al e H), ou a baixa fertilidade em algumas situações é devido a baixa quantidade 1744 de bases no material de origem do solo (Raij 1981).

\section{Conservatismo de nicho}

Analisamos o conservatismo de nicho das variáveis climáticas e edáficas mais importantes para a diferenciação das comunidades arbóreas. Dentre todas as variáveis, apenas soma de bases foi uma característica conservada. Os fenômenos que contribuem para a distribuição de traços e funções são plasticidade fenotípica, sorteio ecológico e seleção natural, que são dependentes da diferenciação ambiental (Ackerly 2003), seja causada por mudanças globais ou por colonização de novos ambientes. Na história recente da Terra, ocorreram diversas flutuações climáticas na ordem de 10 ํㅡ, no entanto, a divergência de várias das espécies encontradas hoje na região Neotropical é do Mioceno (Pennington et al. 2004; Simon et al. 2009), período anterior às flutuações climáticas do Quaternário. Portanto a maioria das espécies presentes no Cerrado deve ser tolerante a uma amplitude climática maior do que a observada para o bioma, já que se mantiveram mesmo após as flutuações climáticas do Pleistoceno. No entanto, nos limites extremos desse gradiente, observamos diferenças de temperatura média anual de $8^{\circ} \mathrm{C}$ e de $1400 \mathrm{~mm}$ de precipitação, que atuam nas mudanças das comunidades. Por outro lado, as díspares condições edáficas, distribuídas em manchas pelo bioma, representaram oportunidade de especialização na exploração dos nutrientes, o que requer mudanças fisiológicas importantes (Gurevitch et al. 2009).

O conservatismo de nicho é caracterizado por maior sobreposição no uso dos recursos por espécies evolutivamente mais próximas, e é causado pela especiação de linhagens evolutivas sob condições pretéritas semelhantes (Prinzing et al. 2001). Considerando que as mudanças das condições do solo do Cerrado são mais lentas ao longo do tempo evolutivo do que o clima, é bastante plausível que algumas linhagens tenham preferências edáficas. Plantas vasculares da Europa também apresentam conservatismo de nicho para variáveis edáficas, sendo que nitrogênio no solo foi explicado principalmente nos níveis taxonômicos mais baixos

1769 (até família) e reação do solo foi explicado em níveis acima de família (Prinzing et al. 2001). No Cerrado algumas famílias são conhecidas por terem ocorrência preferencial em solos férteis

1771 (Torres et al. 1997). No presente estudo, observamos preferências edáficas em níveis de 1772 famílias e até níveis mais altos, como o clado a que pertencem as ordens Malvales e Sapindales, que apresenta predominância de espécies com preferência por altos valores de soma de bases. 
A acumulação de Al é uma característica conhecida para as plantas do Cerrado, em

1775

1776

1777

1778

1779

1780

1781

1782

1783

1784

1785

1786

1787

1788

1789

1790

1791

1792

1793

1794

1795

1796

1797

1798

1799 especial das famílias Melastomataceae e Vochysiaceae (Andrade et al. 2011; Haridasan 1982), o que é uma característica simplesiomórfica da ordem Myrtales (Jansen 2002), que inclui as duas famílias citadas. No entanto não foi observado conservatismo de saturação por alumínio, pois essa característica não pode ser mensurada pelo ambiente, já que a acumulação de Al não está relacionada necessariamente à sua disponibilidade no solo. Estudos dessa natureza teriam um papel importante para a interpretação da história do cerrado.

\section{Consequências para a conservação}

Baseada na limitada capacidade de dispersão das plantas frente às aceleradas mudanças climáticas (Pearson 2006), a perpetuação das espécies do Cerrado é uma questão preocupante, pois não se sabe se as espécies serão capazes de acompanhar essas mudanças. Na Amazônia boliviana espera-se que $65 \%$ das espécies tenham declínio da sua distribuição com o aumento da temperatura e 39\% experimentem esse fenômeno com a intensificação da estação seca (Toledo et al. 2012). Nesse sentido, é importante que estudos populacionais procurem entender o processo de dispersão das espécies.

A procura por solos de alta qualidade para produção agrícola no Cerrado terá consequências irreparáveis para a perda de diversidade filogenética. Os produtores rurais buscam solos mais férteis para o estabelecimento das culturas (Goedert 1989), suprimindo de imediato a vegetação. Num segundo momento, caso essas áreas precisem ser recuperadas, dificilmente as mesmas espécies irão se estabelecer em um solo empobrecido pelas culturas. Isso porque anualmente cerca de $180 \mathrm{Kg} / \mathrm{ha}$ de bases trocáveis são perdidos anualmente (Fageria 1999). A retirada dessas bases representa o empobrecimento e a acidificação do solo, muitas vezes irreversível em decorrência do mal manejo das culturas (Vendrame et al. 2010). Linhagens de plantas que apresentam preferência por solos mais férteis tendem a desaparecer frente a essas expectativas. Apesar das culturas anuais serem fertilizadas a cada ciclo, a alta fertilidade do solo é uma característica procurada, especialmente para pecuária.

Com esse estudo, pudemos observar que os filtros ambientais climáticos e edáficos atuam em diferentes escalas, sendo 'que o clima determina o pool regional de espécies e o solo diferencia as comunidades em escala local, o que possivelmente aumenta a diversidade em nessas escalas. Dentre as variáveis mais importantes, apenas o requerimento por soma de 
bases é uma característica conservada na filogenia, o que tem implicações para a conservação filogenética das comunidades do Cerrado.

\section{AGRADECIMENTOS}

À CAPES, CNPq e Programa Ciência Sem Fronteira pelas bolsas de estudos concedidas a RDF. Ao CNPq pela bolsa de produtividade em pesquisa concedida a RBM. À Expedição AngloBrasileira Xavantina Cachimbo e ao Projeto Conservação e Manejo da Biodiversidade do Bioma Cerrado, pela concessão dos dados de solos. À Rede ComCerrado e Royal Botanic Garden Edinburgh pelo fornecimento da estrutura de laboratório.

\section{REFERÊNCIAS}

Abreu MF, Pinto JRR, Maracahipes L, et al., 2012. Influence of edaphic variables on the floristic composition and structure of the tree-shrub vegetation in typical and rocky outcorp cerrado areas in Serra Negra, Goias State, Brazil. Brazilian Journal of Botany, 35(3):259272.

Ackerly DD, 2003. Community Assembly, Niche Conservatism, and Adaptive Evolution in Changing Environments. International Journal of Plant Sciences, 164(3):164-184.

Amorim PK \& Batalha MA, 2007. Soil-vegetation relationships in hyperseasonal cerrado, seasonal cerrado, and wet grassland in Emas National Park (central Brazil). Acta Oecologica, 32(3):319-327.

Andrade LRM, Barros LMG, Echevarria GF, et al., 2011. Al-hyperaccumulator Vochysiaceae from the Brazilian Cerrado store aluminum in their chloroplasts without apparent damage. Environmental and Experimental Botany, 70:37-42.

APGIII, Chase MW, Fay MF, et al., 2009. An update of the Angiosperm Phylogeny Group classification for the orders and families of flowering plants: APG III. Botanical Journal of the Linnean Society, 161:105-121.

Arens K, 1963. As plantas lenhosas dos campos cerrados como flora adaptada às deficiências minerais do solo. In , Simpósio sobre o Cerrado. . São Paulo, SP: Editora da Universidade de São Paulo, p. 285-303.

Barberi M, Salgado-Labouriau ML \& Suguio K, 2000. Paleovegetation and paleoclimate of "Vereda de Águas Emendadas", central Brazil. Journal of South American ..., 13:241-254.

Batalha MA, Silva IA, Cianciaruso MV, França H \& De Carvalho GH, 2011. Phylogeny, traits, environment, and space in cerrado plant communities at Emas National Park (Brazil). Flora - Morphology, Distribution, Functional Ecology of Plants, 206(11):949-956. 
Blanchet FG, Legendre P \& Borcard D, 2008. Forward selection of explanatory variables. Ecology, 89(9):2623-32.

Bongers F, Poorter L, Van-Rompaey RSAR \& Parren MPE, 1999. Distribution of twelve moist forest canopy tree species in Liberia and Côte d'Ivoire: response curves to a climatic gradient. Journal of Vegetation Science, 10(3):371-382.

Borcard D, Legendre P \& Drapeau P, 1992. Partialling out the spatial component of ecological variation. Ecology, 73(3):1045-1055.

Borges HBN, 2000. Biologia reprodutiva e conservação do estrato lenhoso numa comunidade do cerrado. Tese de Doutorado. Campinas, SP: PPG Biologia Vegetal, Universidade Estadual de Campinas.

Bucci SJ, Goldstein G, Meinzer FC, et al., 2004. Mechanisms contributing to seasonal homeostasis of minimum leaf water potential and predawn disequilibrium between soil and plant water potential in Neotropical savanna trees. Trees, 19(3):296-304.

Bueno ML, Neves DR, Oliveira-Filho AT, Lehn CR \& Ratter JA, 2013. a Study in an Area of Transition Between Seasonally Dry Tropical Forest and Mesotrophic Cerradão, in Mato Grosso Do Sul, Southwestern Brazil. Edinburgh Journal of Botany, 70(03):469-486.

Bueno ML, Rafael D, Neves $M$, et al., 2013. Influence of edaphic factors on the floristic composition of an area of cerradão in the Brazilian central-west. Acta Botânica Brasílica, 27(2):445-455.

Camargo AP, 1963. Clima do Cerrado. In , Simpósio sobre o Cerrado. . São Paulo, SP: Editora da Universidade de São Paulo, p. 93-115.

Castro AAJF, 1994. Comparação florístico-geográfica (Brasil) e fitossociologia (Piauíi - São Paulo) de amostras de cerrado. Instituto de Biologia. Campinas, SP: Universidade Estadual de Campinas.

Cavender-Bares J, Kozak KH, Fine PV a \& Kembel SW, 2009. The merging of community ecology and phylogenetic biology. Ecology letters, 12(7):693-715.

Clark DB, Palmer MW \& Clark DA, 1999. Edaphic factors and the landscape-scale distributions of tropical rain forest trees. Ecology, 80(8):2662-2675.

Clements FE \& Shelford V, 1939. Bio-ecology. New York, USA / London, UK: John Wiley \& Sons / Chapman \& Hall, p. 425.

Diniz-Filho JAF, Santos T, Rangel TF \& Bini LM, 2010. A comparison of metrics for estimating phylogenetic signal under alternative evolutionary models. 679(2012):673-679.

Dray S, Legendre P \& Blanchet G, 2011. Packfor: Forward Selection with permutation (Canoco p.46). R package version 0.0-8/r100., (http://r-forge.r-project.org/projects/sedar/). 
Duarte MC, Esteves GL, Salatino MLF, Walsh KC \& Baum D a., 2011. Phylogenetic Analyses of $<1>$ Eriotheca $</ 1>$ and Related Genera (Bombacoideae, Malvaceae). Systematic Botany, $36(3): 690-701$.

Durigan G \& Ratter JA, 2006. Successional Changes in Cerrado and Cerrado/Forest Ecotonal Vegetation in Western São Paulo State, Brazil, 1962-2000. Edinburgh Journal of Botany, 63(01):119.

Eiten G, 1972. The Cerrado vegetation of Brazil. The Botanical Review, 38(2):201-341.

Engelbrecht BMJ, Comita LS, Condit R, et al., 2007. Drought sensitivity shapes species distribution patterns in tropical forests. Nature, 447(7140):80-2.

Fageria NK, 1999. Resposta de arroz de terras altas, feijão, milho e soja à saturação por base em solo de cerrado. :416-424.

Felfili JM, Carvalho FA \& Haidar RF, 2005. Manual para o monitoramento de parcelas permanentes nos biomas Cerrado e Pantanal. Brasília, DF: Universidade de Brasília, Departamento de Engenharia Florestal.

Felfili JM, Silva-Júnior MC, Filgueiras TS, et al., 1994. Projeto biogeografia do bioma cerrado: vegetação e solos. Cadernos de Geociências, 12(4):75-167.

Ferraz-Vicentini KR \& Salgado-Labouriau ML, 1996. Palynological analysis of a palm swamp in Central Brazil. Journal of South American Earth Sceinces, 9(3-4):207-219.

Ferri MG \& Coutinho LM, 1958. Contribuição ao conhecimento da ecologia do cerrado. Estudo comparativo da economia d'água de sua vegetacao, em Emas (Est. de São Paulo), Campo Grande (Est. de Mato Grosso) e Goiânia (Est. de Goiás). Boletim da Faculdade de Filosofia, Ciências e Letras, Universidade de São Paulo. Botânica, 12:103-150.

Franco AC, 2002. Ecophysiology of wood plants. In PS Oliveira \& RJ Marquis (eds.), The Cerrados of Brazil: ecology and natural history of a neotropical savanna. . Columbia University Press, p. 178-198.

Furley P, 2006. Tropical savannas. Progress in Physical Geography, 30(1):105-121.

Furley P \& Ratter JA, 1988. Soil resources and plant communities of the central brazilian cerrado and their development. Journal of Biogeography, 15(1):97-108.

Goedert WJ, 1989. Região dos Cerrados: potencial agrícola e política para seu desenvolvimento. Pesquisa Agropecuária Brasileira, 24(1):1-17.

Goodland R \& Pollard R, 1973. The Brazilian Cerrado vegetation: a fertility gradient. Journal of Ecology, 61(1):219-224.

Greve M, Lykke AM, Blach-Overgaard A \& Svenning J-C, 2011. Environmental and anthropogenic determinants of vegetation distribution across Africa. Global Ecology and Biogeography, 20(5):661-674. 
Grinnell J, 1917. The niche-relationships of the California thrasher. Auk, 34:427-433.

Gurevitch J, Scheiner SM \& Fox GAGA, 2009. Ecologia Vegetal. 2a ed., São Paulo, SP: Artmed, p. 592.

Haridasan M, 1982. Aluminium accumulation by some cerrado native species of central. Plant and Soil, 65:265-273.

Haridasan M, 2000. Nutrição mineral das plantas nativas do cerrado. Revista Brasileira de Fisiologia Vegetal, 12(1):54-64.

Haridasan M, 2008. Nutritional adaptations of native plants of the cerrado biome in acid soils. Brazilian Journal Of Plant Physiology, 20(3):183-195.

Hijmans RJ, Cameron S, Parra J, Jones P \& Jarvis A. The WorldClim interpolated global terrestrial climate surfaces. (http://www.worldclim.org).

Holmgren M \& Poorter L, 2007. Does a ruderal strategy dominate the endemic flora of the West African forests? Journal of Biogeography, 34(6):1100-1111.

Hubbell SP, 2001. The unified neutral theory of Biodiversity and Biogeography. Princeton, New Jersey: Princeton University Press, p. 375.

Hutchinson GE, 1957. Population studies - animal ecologyand demography - concluding remarks. Cold Spring Harbor Symposia on Quantitative Biology, 22:415-427.

Jansen S, 2002. Aluminium Accumulation in Leaves of 127 Species in Melastomataceae, with Comments on the Order Myrtales. Annals of Botany, 90(1):53-64.

JBRJ. Lista de Espécies da Flora do Brasil. (http://floradobrasil.jbrj.gov.br/).

John R, Dalling JW, Harms KE, et al., 2007. Soil nutrients influence spatial distributions of tropical tree species. Proceedings of the National Academy of Sciences of the United States of America, 104(3):864-9.

Kriticos DJ, Webber BL, Leriche A, et al., 2012. CliMond: global high-resolution historical and future scenario climate surfaces for bioclimatic modelling. Methods in Ecology and Evolution, 3(1):53-64.

Kubota Y, Hirao T, Fujii S, Shiono T \& Kusumoto B, 2014. Beta diversity of woody plants in the Japanese archipelago: the roles of geohistorical and ecological processes. Journal of Biogeography, 41(7):1767-1276.

Ledru M, 1993. Late Quaternary Environmental and Climatic Changes in Central Brazil. Quaternary Research, 39:90-98.

Ledru M-P, Salgado-Labouriau ML \& Lorscheitter ML, 1998. Vegetation dynamics in southern and central Brazil during the last 10,000 yr B.P. Review of Palaeobotany and Palynology, 99(2):131-142. 
Lehmann CER, Anderson TM, Sankaran M, et al., 2014. Savanna Vegetation-Fire-Climate Relationships Differ Among Continents. Science, 343(January):548-552.

Leibold MA, Economo EP \& Peres-Neto P, 2010. Metacommunity phylogenetics: separating the roles of environmental filters and historical biogeography. Ecology Letters, 13(10):12901299.

Maddison WP \& Maddison DR, 2014. Mesquite: A modular system for evolutionary analysis. University of British Columbia \& Oregon State University, (http://mesquiteproject.wikispaces.com/).

Marimon-Júnior BH \& Haridasan M, 2005. Comparação da vegetação arbórea e características edáficas de um cerradão e um cerrado sensu stricto em áreas adjacentes sobre solo distrófico no leste de Mato Grosso, Brasil. Acta Botanica Brasilica, 19(4):913-926.

Mckenzie D, Peterson DW, Peterson DL \& Seattle PET, 2003. Climatic and biophysical controls on conifer species distributions in mountain forests of Washington State, USA. Journal of Biogeography, 30(7):1093-1108.

Moreno MIC, Schiavini I \& Haridasan M, 2008. Fatores edáficos influenciando na estrutura de fitofisionomias do cerrado. Caminhos de Geografia, 9(25):173 - 194.

Myers N, Mittermier RA, Mittermier CG, et al., 2000. Biodiversity hotspots for conservation priorities. Nature, 403(24):853-858.

Neri AV, Schaefer CEGR, Silva a. F, et al., 2012. the Influence of Soils on the Floristic Composition and Community Structure of an Area of Brazilian Cerrado Vegetation. Edinburgh Journal of Botany, 69(01):1-27.

Oksanen J, Blanchet FG, Kindt R, et al., 2014. Community Ecology Package. R Package, version 2.0-10, (http://cran.r-project.org/web/packages/vegan/vegan.pdf).

Pearson RG, 2006. Climate change and the migration capacity of species. Trends in Ecology and Evolution, 21(3):111-113.

Pearson RG \& Dawson T, 2003. Predicting the impacts of climate change on the distribution of species: are bioclimate envelope models useful? Global ecology and biogeography, 12:361-371.

Pennington RT, 2003. Monography of Andira (Leguminosae-Papilonoideae. Systematic Botany Monographs, 64:1-143.

Pennington RT, Lavin M, Prado DE, et al., 2004. Historical climate change and speciation: neotropical seasonally dry forest plants show patterns of both tertiary and quaternary diversification. Philosophical transactions of the Royal Society of London. Series B, Biological sciences, 359:515-37.

Potts MD, Ashton PS, Kaufman LS \& Plotkin JB, 2002. Habitat patterns in tropical rain forests: a comparision of 105 plots in northwest Borneo. Ecology, 83(10):2782-2797. 
Prentice IC, Cramer W, Harrison SP, et al., 1992. Special paper: a global biome model based on plant physiology and dominance, soil properties and climate. Journal of Biogeography, 19(2):117-134.

Prinzing a, Durka W, Klotz S \& Brandl R, 2001. The niche of higher plants: evidence for phylogenetic conservatism. Proceedings. Biological sciences / The Royal Society, 268(1483):2383-9.

Punyasena SW, Eshel G \& McElwain JC, 2008. The influence of climate on the spatial patterning of Neotropical plant families. Journal of Biogeography, 35:117-130.

Raij B Van, 1981. Avaliação da fertilidade do solo. 1st ed., Piracicaba, SP: Instituto da Potassa \& Fosfato: Instituto Internacional da Potassa, p. 142.

Ranzani G, 1963. Solos do Cerrado. In E da U de S Paulo (ed.), Simpósio sobre o Cerrado. . São Paulo, SP, p. 423.

Ratter JA \& Dargie TCD, 1992. An analysis of the floristic composition of 26 cerrado ares in Brazil. Edinburgh Journal of Botany, 49(02):235.

Ratter JA, Richards PW, Argent G \& Gifford DR, 1973a. Observation on the vegetation of northeastern Mato Grosso: I. The Woody vegetation types of the Xavantina-Cachimbo Expedition Area. Philosophical transactions of the Royal Society of London. Series B, Biological sciences, 266(880):449-492.

Ratter JA, Richards PW, Argent G \& Gifford DR, 1973b. Observations on the vegetation of north eastern Mato Grosso, 1. The woody vegetation types of the Xavantina-Cachimbo expedition area. Phylosophical Transactions of the Royal Society, 226:449-492.

Revell LJ, 2012. phytools: an R package for phylogenetic comparative biology (and other things). Methods in Ecology and Evolution, 3(2):217-223.

Ribeiro JF \& Walter BMT, 1998. Fitofisionomias do Bioma Cerrado. In SM Sano \& SP Almeida (eds.), Cerrado: ambiente e flora. . Planaltina-DF: EMBRAPA-CPAC.

Ribeiro JF \& Walter BMT, 2008. As principais fitofisionomias do bioma Cerrado. In SM Sano SP Almeida \& JF Ribeiro (eds.), Cerrado: ecologia e flora. . Brasília, DF: EMBRAPA, p. 151-212.

Ruggiero PGC, Batalha MA, Pivello VR \& Meirelles ST, 2002. Soil-vegetation relationships in cerrado (Brazilian savanna) and semideciduous forest, Southeastern Brazil. Plant Ecology, 160:1-16.

Salgado-Labouriau ML, 1997. Late quaternary palaeoclimate in the savannas of South America. Journal of Quaternary Science, 12(5):371-379.

Schimel DS, VEMAP \& Braswell BH, 1997. Continental scale variability in ecosystem processes: models, data, and the role of disturbance. Ecological Monographs, 67(2):251-271. 
Silva DM \& Batalha MA, 2008. Soil-vegetation relationships in cerrados under different fire frequencies. Plant and Soil, 311(1-2):87-96.

Silva IA \& Batalha MA, 2009. Woody plant species co-occurrence in Brazilian savannas under different fire frequencies. Acta Oecologica, 36(1):85-91.

Simon MF, Grether R, De Queiroz LP, et al., 2009. Recent assembly of the Cerrado, a neotropical plant diversity hotspot, by in situ evolution of adaptations to fire. Proceedings of the National Academy of Sciences of the United States of America, 106(48):20359-64.

Skarpe C, 1996. Plant functional types and climate in a southern African savanna. Journal of Vegetation Science, 7:397-404.

Stephenson NL, 1990. Climatic control of vegetation distribution: The role of the water balance. The American Naturalist, 135(5):649-670.

Toledo M, Peña-Claros M, Bongers F, et al., 2012. Distribution patterns of tropical woody species in response to climatic and edaphic gradients. Journal of Ecology, 100(1):253-263.

Torres RB, Roberto F \& Kinoshita LS, 1997. Climate, soil and tree flora relationships in forests in the state of São Paulo, southeastern Brasil. Brazilian Journal of Botany, 20(1):41-49.

Vendrame PRS, Brito OR, Guimarães MF, Martins ÉS \& Becquer T, 2010. Fertility and acidity status of latossolos (oxisols) under pasture in the Brazilian Cerrado. Anais da Academia Brasileira de Ciencias, 82(4):1085-1094.

Werneck FP, Nogueira CC, Colli GR, Sites JW \& Costa GC, 2012. Climatic stability in the Brazilian Cerrado: implications for biogeographical connections of South American savannas, species richness and conservation in a biodiversity hotspot. Journal of Biogeography, 39(9):1695-1706. 


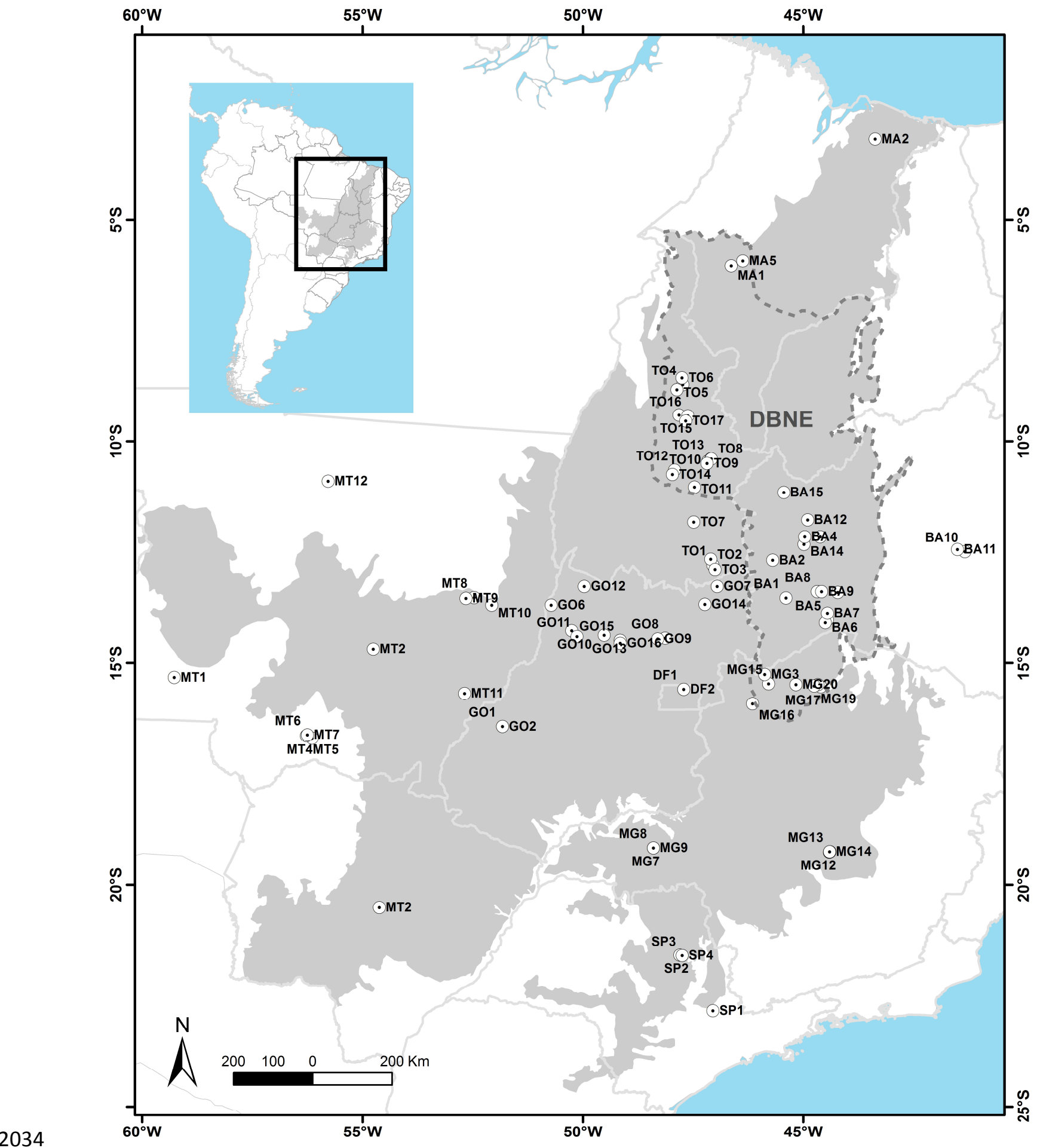

2035 Figura 1. Localidades no bioma Cerrado onde foram realizados levantamentos florísticos e 2036 coletas de solo. Para as mesmas localidades foram extraídas informações climáticas baseadas 2037 em temperatura, precipitação, radiação e umidade (Kriticos et al., 2012). 
Escala 1

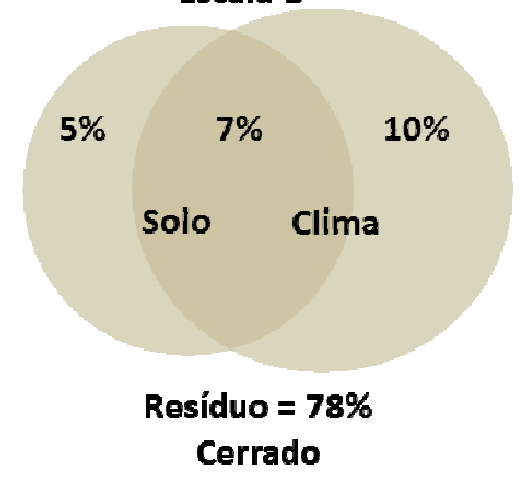

2039

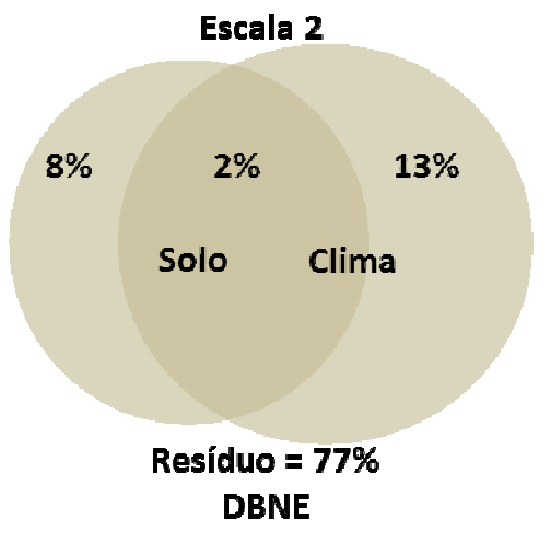

Escala 3

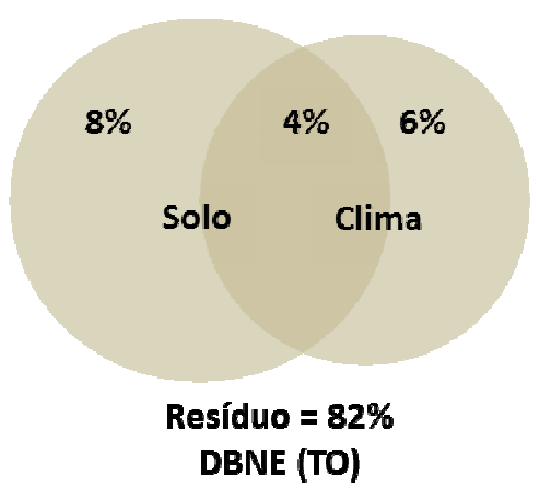

2040 Figura 2. Contribuição dos diferentes conjuntos de variáveis ambientais para a composição das 2041 comunidades arbóreas do cerrado em três escalas: (1) bioma Cerrado; (2) Distrito Biogeográfico 2042 Nordeste (DBNE); e (3) localidades do estado do Tocantins dentro do DBNE. Resultados 2043 baseados na partição de variância entre dados climáticos, edáficos e espaciais na explicação da 2044 composição de 87 comunidades arbóreas do Cerrado. 


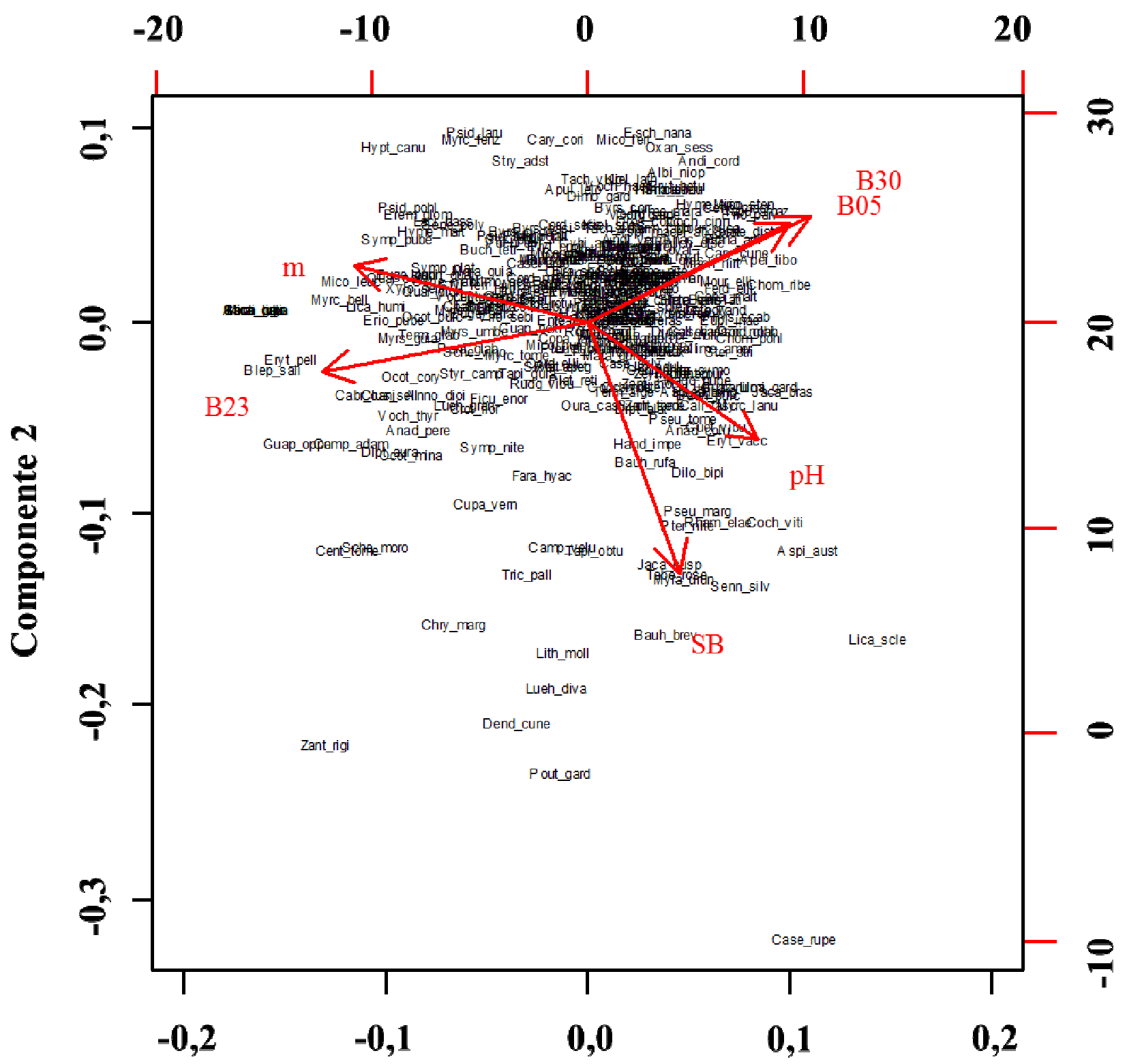

Componente 1

2047 Figura 3. Influência das variáveis ambientais temperatura máxima da semana mais quente (B5), 2048 sazonalidade da radiação (B23), menor índice de umidade semanal (B30), soma de bases (SB), 2049 saturação por Alumínio $(\mathrm{m})$ e acidez do solo (pH em água) em relação à distribuição das 303 2050 espécies lenhosas de 87 comunidades de Cerrado sentido amplo. 


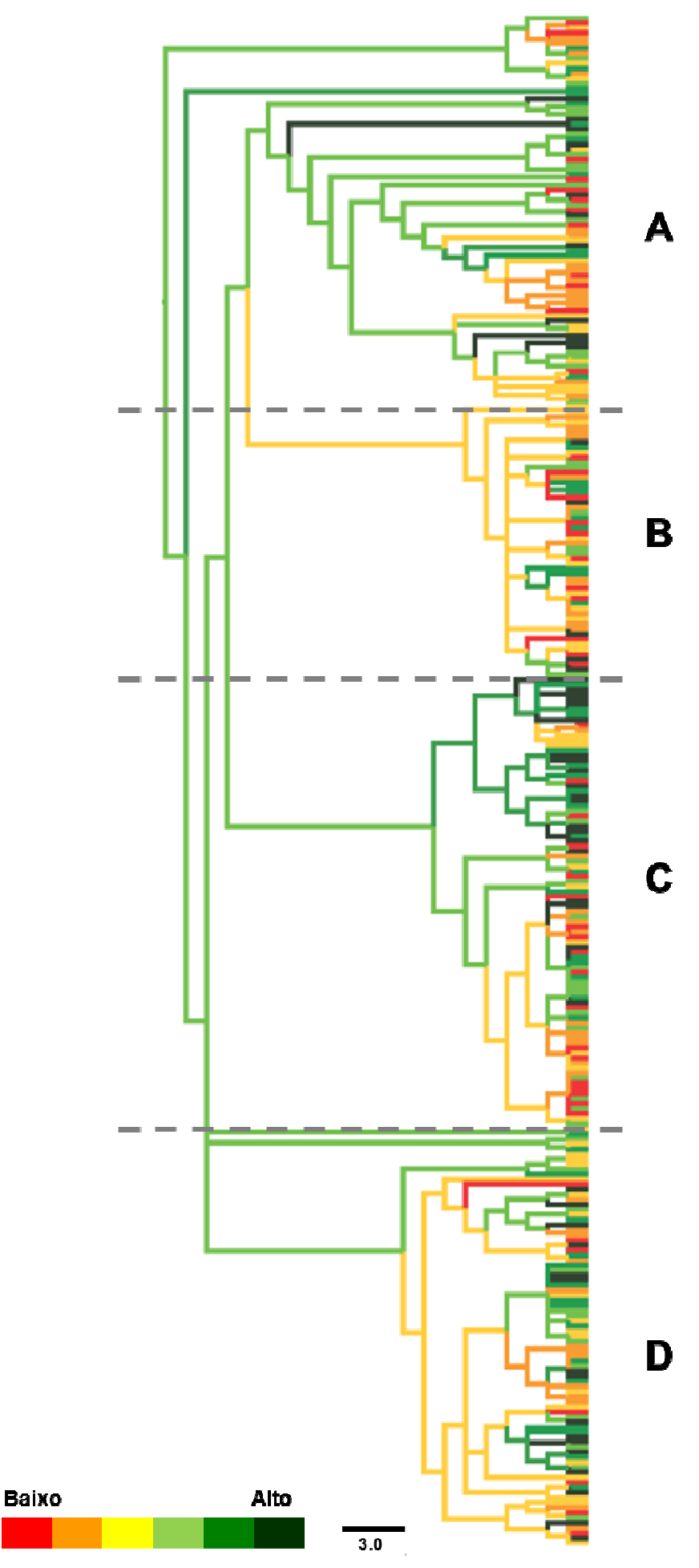

2053 Figura 4. Hipótese filogenética para as espécies lenhosas de 87 comunidades do cerrado 2054 sentido amplo baseada em filogenias pré-existentes. As cores correspondem à reconstrução do 2055 estado ancestral baseado em parcimônia por preferência à quantidade de somas de bases 2056 presente no solo. As divisões (A, B, C e D) referem-se às partes do cladograma analisadas 2057 individualmente. 


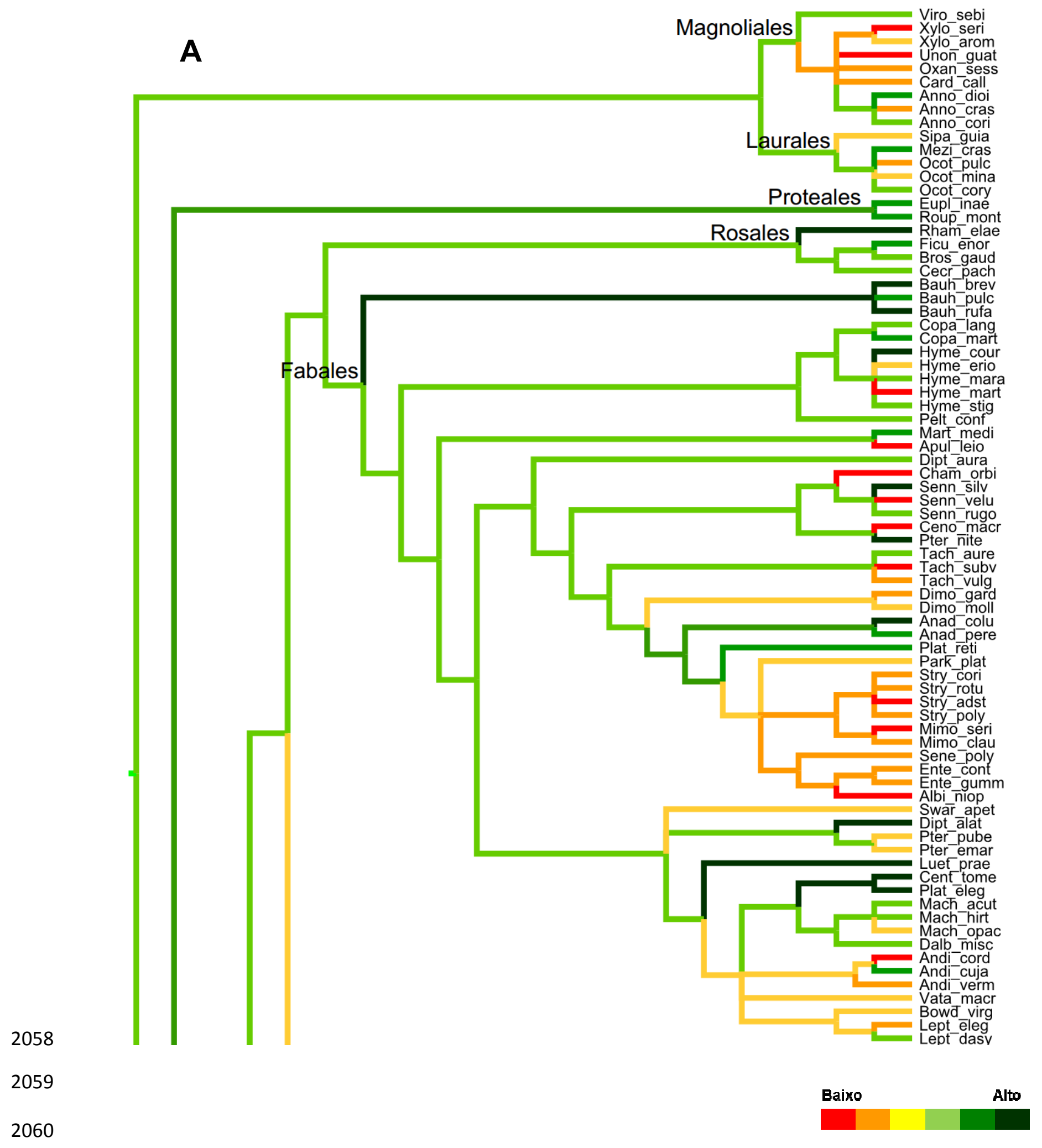

2061 Figura 5. Reconstrução do estado ancestral baseado em parcimônia por preferência à 2062 quantidade de somas de bases presente no solo das espécies lenhosas do Cerrado 2063 pertencentes às ordens (A) Magnoliales, Laurales, Proteales, Rosales e Fabales. As cores 2064 correspondem à reconstrução do estado ancestral baseado em parcimônia por preferência à 2065 quantidade de somas de bases presente no solo. As siglas das espécies podem ser consultadas 2066 em MS1. 

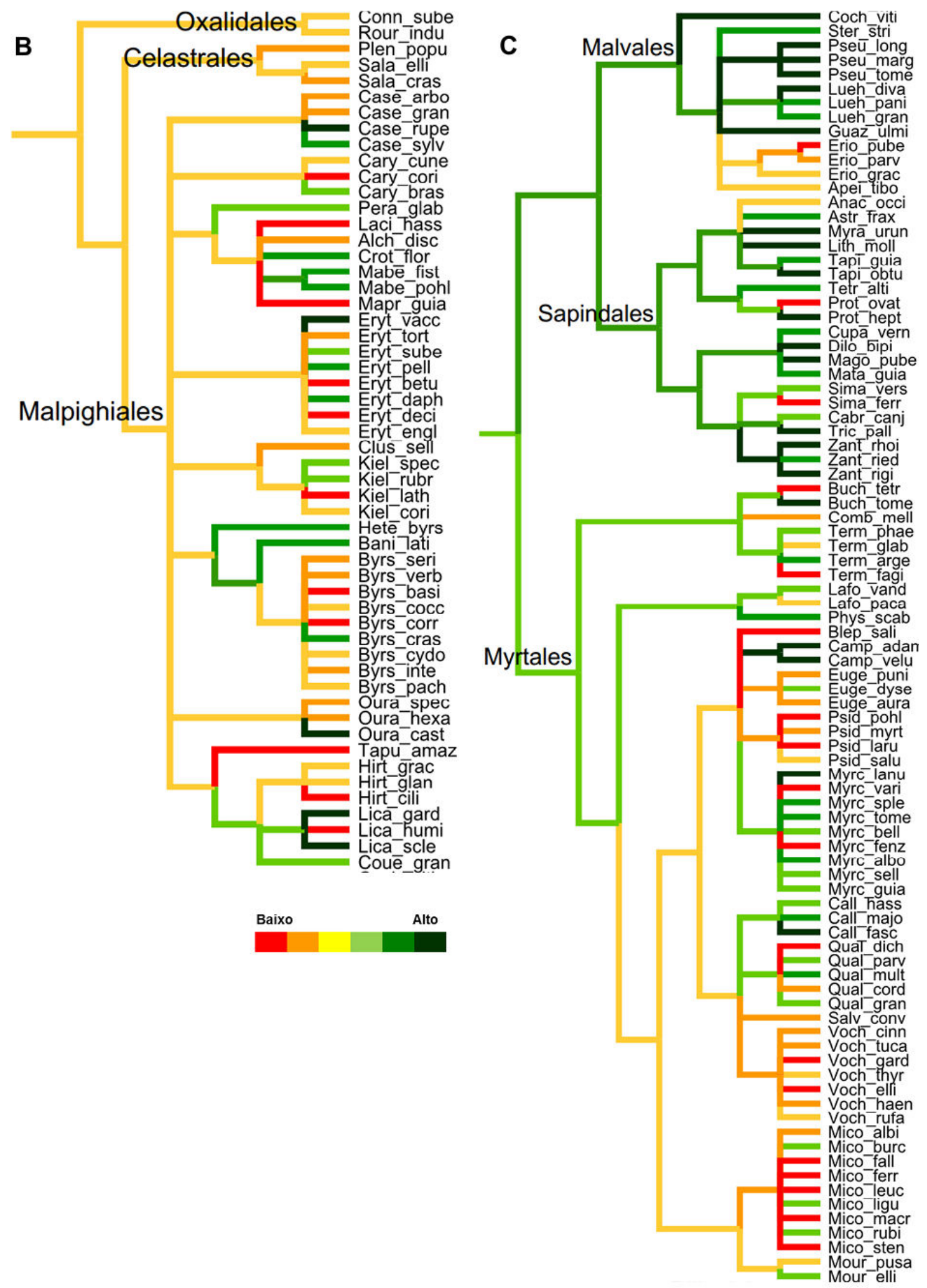

Figura 6. Reconstrução do estado ancestral baseado em parcimônia por preferência à 2069 quantidade de somas de bases presente no solo das espécies lenhosas do Cerrado 2070 pertencentes às ordens (B) Oxalidades, Celastrales, Malpighales; e (C) Malvales, Sapindales e 2071 Myrtales. As cores correspondem à reconstrução do estado ancestral baseado em parcimônia 2072 por preferência à quantidade de somas de bases presente no solo. As siglas das espécies 2073 podem ser consultadas em MS1. 
D

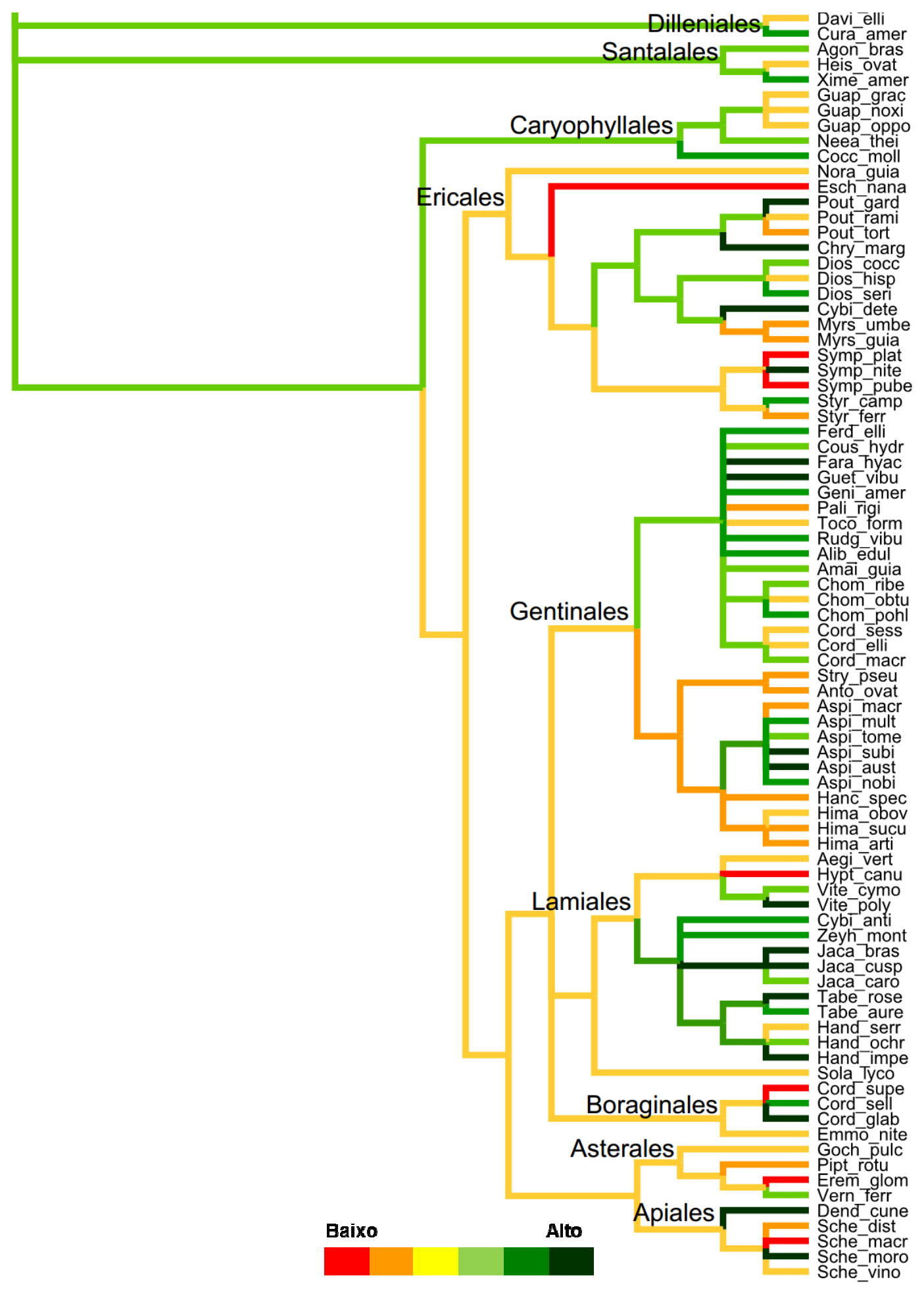

2076 Figura 7. Reconstrução do estado ancestral baseado em parcimônia por preferência à 2077 quantidade de somas de bases presente no solo das espécies lenhosas do Cerrado 2078 pertencentes às ordens (D) Dilleniales, Santalales, Caryophyllales, Ericales, Gentiales, Lamiales, 2079 Boraginales, Asterales e Apiales. As cores correspondem à reconstrução do estado ancestral. As 2080 siglas das espécies podem ser consultadas em MS1. 
2083 Tabela1. Variáveis bioclimáticas e edáficas usadas na seleção progressiva a priori da partição de 2084 variância (Vpar).

\begin{tabular}{llll}
\hline Sigla & Variáveis climáticas & Sigla & Variáveis edáficas \\
\hline Bio04 & Sazonalidade da temperatura (coef. de var.) & $\mathrm{m}$ & Saturação por Alumínio (\%) \\
Bio05 & Temperatura máxima da semana mais quente $\left({ }^{\circ} \mathrm{C}\right)$ & $\mathrm{K}$ & Saturação por Potássio (\%) \\
Bio06 & Temperatura mínima da semana mais fria $\left({ }^{\circ} \mathrm{C}\right)$ & $\mathrm{Ca}$ & Saturação por Cálcio (\%) \\
Bio12 & Precipitação anual $(\mathrm{mm})$ & $\mathrm{Mg}$ & Saturação por Magnésio $(\%)$ \\
Bio15 & Sazonalidade da precipitação & $T$ & Capacidade de troca catiônica $\left(\mathrm{cmolc} / \mathrm{dm}^{3}\right)$ \\
Bio17 & Precipitação do trimestre menos chuvoso $(\mathrm{mm})$ & $\mathrm{pH}$ & Acidez do solo (pH em água) \\
Bio20 & Radiação média anual (W m-2) & $\mathrm{V}$ & Saturação por bases $(\%)$ \\
Bio23 & Sazonalidade da radiação & Argila & Percentual de argila no solo $(\%)$ \\
Bio29 & Máximo índice de umidade semanal & Silte & Percentual de silte no solo $(\%)$ \\
Bio30 & Mínimo índice de umidade semanal & Areia & Percentual de areia no solo $(\%)$ \\
Bio31 & Sazonalidade do índice de umidade & SB & Soma de bases trocáveis $\left(\mathrm{cmolc} / \mathrm{dm}^{3}\right)$ \\
\hline
\end{tabular}


Tabela 2. Variáveis climáticas e edáficas selecionadas a priori por seleção progressiva para análise de partição de variância para três escalas no bioma Cerrado. Escala 1: bioma Cerrado; escala 2: Distrito Biogeográfico Nordeste (DBNE); escala 3: comunidades localizadas no estado do Tocantins dentro do DBNE.

\begin{tabular}{|c|c|c|c|c|c|c|c|c|c|c|c|c|}
\hline & \multicolumn{6}{|c|}{ Clima } & \multicolumn{6}{|c|}{ Solos } \\
\hline & Var. & $R^{2}$ & $\begin{array}{c}\mathrm{R}^{2} \\
\text { cum. }\end{array}$ & $\begin{array}{l}\mathrm{R}^{2} \text { cum. } \\
\text { aj. }\end{array}$ & $\mathbf{F}$ & $\mathbf{p}$ & Var. & $\mathbf{R}^{2}$ & $\begin{array}{l}\mathbf{R}^{2} \\
\text { cum. }\end{array}$ & $\begin{array}{l}\mathrm{R}^{2} \text { cum. } \\
\text { aj. }\end{array}$ & $\mathbf{F}$ & $\mathbf{p}$ \\
\hline \multirow{8}{*}{ Escala 1} & Bio05 & 0.060 & 0.060 & 0.050 & 5.370 & 0.001 & $\mathrm{~m}$ & 0.050 & 0.050 & 0.030 & 4.04 & 0.001 \\
\hline & Bio17 & 0.050 & 0.110 & 0.080 & 4.300 & 0.001 & SB & 0.040 & 0.090 & 0.070 & 4.06 & 0.001 \\
\hline & Bio12 & 0.040 & 0.150 & 0.110 & 3.940 & 0.001 & $\mathrm{~K}$ & 0.030 & 0.120 & 0.090 & 2.89 & 0.001 \\
\hline & Bio23 & 0.030 & 0.170 & 0.130 & 2.760 & 0.001 & $\mathrm{pH}$ & 0.020 & 0.140 & 0.100 & 2.28 & 0.001 \\
\hline & Bio20 & 0.020 & 0.190 & 0.140 & 2.020 & 0.001 & Areia & 0.020 & 0.160 & 0.110 & 1.81 & 0.003 \\
\hline & Bio06 & 0.020 & 0.210 & 0.150 & 2.020 & 0.002 & $t$ & 0.020 & 0.180 & 0.120 & 1.58 & 0.014 \\
\hline & Bio29 & 0.020 & 0.230 & 0.160 & 1.890 & 0.001 & & & & & & \\
\hline & Bio15 & 0.020 & 0.250 & 0.170 & 1.830 & 0.002 & & & & & & \\
\hline \multirow{4}{*}{ Escala 2} & Bio23 & 0.117 & 0.117 & 0.090 & 4.250 & 0.001 & $t$ & 0.065 & 0.065 & 0.036 & 2.22 & 0.001 \\
\hline & Bio12 & 0.048 & 0.165 & 0.111 & 1.784 & 0.003 & $\mathrm{pH}$ & 0.055 & 0.120 & 0.063 & 1.93 & 0.005 \\
\hline & Bio20 & 0.047 & 0.212 & 0.133 & 1.777 & 0.003 & $\mathrm{Ca}$ & 0.050 & 0.170 & 0.087 & 1.82 & 0.014 \\
\hline & Bio04 & 0.038 & 0.250 & 0.146 & 1.455 & 0.034 & & & & & & \\
\hline Escala 3 & Bio29 & 0.174 & 0.174 & 0.099 & 2.317 & 0.008 & $\mathrm{pH}$ & 0.198 & 0.198 & 0.125 & 2.71 & 0.005 \\
\hline
\end{tabular}

2091

2092 
2093 Tabela 3. Resultado da análise de partição da variância. A composição de 87 comunidades de 2094 espécies lenhosas em cerrado sentido amplo é explicada por variáveis climáticas (X1) e edáficas 2095 (X2).

\begin{tabular}{|c|c|c|c|c|c|c|c|c|c|}
\hline \multirow{4}{*}{ VARIÁVEIS } & \multirow{2}{*}{\multicolumn{3}{|c|}{$\begin{array}{l}E 2 F_{14,72}=2,72 ; p=0,001 \\
X 1 F 8,72=3,43 ; p=0,001 \\
X 2 F 6,72=1,76 ; p=0,001\end{array}$}} & \multirow{2}{*}{\multicolumn{3}{|c|}{$\begin{array}{l}E 2 F_{8,25}=2,23 ; p=0,001 \\
X 1 F 4,25=2,67 ; p=0,001 \\
X 2 F 4,25=1,79 ; p=0,001\end{array}$}} & \multirow{2}{*}{\multicolumn{3}{|c|}{$\begin{array}{l}E 3 F_{2,8}=2,32 ; p=0,001 \\
X 1 F 1,10=2,55 ; p=0,004 \\
X 2 F 1,10=2,09 ; p=0,017\end{array}$}} \\
\hline & & & & & & & & & \\
\hline & & \multicolumn{3}{|c|}{ GL $\quad R^{2} \quad R^{2}$ ajust. } & \multicolumn{3}{|c|}{$R^{2} \quad R^{2}$ aiuct } \\
\hline & & & & & & $n$ djuse. & & & \\
\hline$[a+d+f+g]=X 1$ & 8 & 0.25 & 0.17 & 4 & 0.25 & 0.15 & 1 & 0.17 & 0.10 \\
\hline$[b+d+e+g]=X 2$ & 6 & 0.18 & 0.12 & 4 & 0.21 & 0.10 & 1 & 0.20 & 0.12 \\
\hline$[a+b+d+e+f+g]=X 1+X 2$ & 14 & 0.35 & 0.22 & 8 & 0.42 & 0.23 & 2 & 0.32 & 0.18 \\
\hline \multicolumn{10}{|l|}{ Frações individuais } \\
\hline$[a]=X 1 \mid X 2$ & 8 & & 0.10 & 4 & & 0.13 & 1 & & 0.06 \\
\hline [b] & 0 & & 0.07 & 0 & & 0.02 & 0 & & 0.04 \\
\hline$[c]=X 2 \mid X 1$ & 6 & & 0.05 & 4 & & 0.08 & 1 & & 0.08 \\
\hline$[\mathrm{h}]=$ Resíduo & & & 0.78 & & & 0.77 & & & 0.82 \\
\hline
\end{tabular}

2096

2097

2098 
2099 Tabela 4. Seleção de modelos por critério de Akaike para as variáveis climáticas e edáficas como 2100 parâmetros explicativos para a composição de 87 comunidades de espécies lenhosas em 2101 cerrado sentido amplo.

\begin{tabular}{|c|c|c|c|c|c|c|c|c|}
\hline & Var. & \multirow{2}{*}{$\frac{\text { GL }}{\text { Bio3 }}$} & \multicolumn{2}{|c|}{ AIC } & $\mathbf{F}$ & & \multicolumn{2}{|l|}{$\mathbf{p}$} \\
\hline \multicolumn{2}{|c|}{$\begin{array}{l}\mathrm{Y} \sim \mathrm{Bio} 05+ \\
\mathrm{AIC}=472.8\end{array}$} & & Bio & $3+$ Bio12 & $+\mathrm{Bio} 20+\mathrm{B}$ & $\frac{\text { N.Perm }}{\text { 3io04 + Bio15 }}$ & & \\
\hline- & Bio15 & & 1 & 472.88 & 1.86 & 199 & 0.005 & $* *$ \\
\hline+ & Bio31 & & 1 & 473.09 & 1.60 & 199 & 0.005 & $* *$ \\
\hline+ & Bio06 & & 1 & 473.13 & 1.57 & 199 & 0.005 & $* *$ \\
\hline+ & Bio29 & & 1 & 473.14 & 1.56 & 199 & 0.005 & $* *$ \\
\hline+ & Bio17 & & 1 & 473.4 & 1.32 & 199 & 0.010 & $* *$ \\
\hline- & Bio30 & & 1 & 473.42 & 2.36 & 199 & 0.005 & $* *$ \\
\hline - & Bio04 & & 1 & 473.48 & 2.42 & 199 & 0.005 & $* *$ \\
\hline+ & alt & & 1 & 473.53 & 1.20 & 199 & 0.070 & . \\
\hline- & Bio20 & & 1 & 473.75 & 2.67 & 199 & 0.005 & $* *$ \\
\hline - & Bio23 & & 1 & 473.79 & 2.71 & 199 & 0.005 & $* *$ \\
\hline - & Bio05 & & 1 & 474.09 & 2.99 & 199 & 0.005 & $* *$ \\
\hline- & Bio12 & & 1 & 474.11 & 3.01 & 199 & 0.005 & $* *$ \\
\hline \multicolumn{9}{|c|}{$Y \sim m+p H+S B ; A I C=478.35$} \\
\hline+ & Areia & & 1 & 478.36 & 1.90 & 199 & 0.005 & $* *$ \\
\hline- & SB & & 1 & 478.65 & 2.22 & 199 & 0.005 & $* *$ \\
\hline- & $\mathrm{pH}$ & & 1 & 478.67 & 2.24 & 199 & 0.005 & $* *$ \\
\hline+ & $\mathrm{K}$ & & 1 & 478.73 & 1.55 & 199 & 0.010 & $* *$ \\
\hline+ & $t$ & & 1 & 478.82 & 1.46 & 199 & 0.010 & $* *$ \\
\hline+ & $\mathrm{Ca}$ & & 1 & 478.83 & 1.44 & 199 & 0.005 & $* *$ \\
\hline+ & Mg & & 1 & 479.18 & 1.11 & 99 & 0.170 & \\
\hline- & $m$ & & 1 & 480.03 & 3.58 & 199 & 0.005 & $* *$ \\
\hline
\end{tabular}

2102 
2104 Tabela 5. Testes de sinal filogenético usando as estatísticas $\mathrm{K}$ de Blomberg e $\lambda$ de Pagel, para 2105 preferência de hábitat usando variáveis climáticas e edáficas selecionadas por critério de 2106 Akaike. Bio05 - temperature máxima da semana mais quente; Bio23 - sazonalidade da radiação; 2107 Bio30 - Menor índice de umidade semanal; SB - soma de bases; $\mathrm{m}$ - saturação por Alumínio; $2108 \mathrm{pH}-$ acidez do solo.

\begin{tabular}{lrrrrrr}
\hline Teste & \multicolumn{1}{c}{ Bio05 } & Bio23 & Bio30 & SB & m & pH \\
\hline Estatística & $4.84 \mathrm{E}-05$ & $1.1 \mathrm{E}-02$ & $6.8 \mathrm{E}-05$ & $5.0 \mathrm{E}-05$ & $4.4 \mathrm{E}-02$ & 0.004 \\
$<$ LogL & -700.99 & 111.35 & 476.15 & -432.07 & -26.53 & -81.50 \\
Log0 & -700.98 & 111.07 & 476.16 & -432.06 & -27.63 & -81.53 \\
$\mathbf{p}$ & 1 & 0.456 & 1 & 1 & 0.138 & 0.807 \\
$\mathbf{Y}$ & 0.097 & 0.086 & 0.090 & 0.108 & 0.098 & 0.102 \\
$\mathbf{p}$ & 0.308 & 0.855 & 0.558 & 0.029 & 0.193 & 0.073 \\
\hline
\end{tabular}


2110 Espécies registradas em 87 localidades de cerrado sentido amplo, distribuídas em 161 gêneros, 58 famílias e 21 ordens. Suas associações às 2111 variáveis ambientais temperatura máxima (Bio05), sazonalidade da radiação (Bio23), menor índice de umidade anual (Bio31), soma de bases (SB), 2112 saturação por Alumínio $(\mathrm{m})$ e acidez do solo ( $\mathrm{pH}$ em água) estão representadas em porcentagem, referente ao intervalo dos valores médios por 2113 localidade de ocorrência das espécies. As classes (A a H) representam classes de associação das espécies em relação à soma de bases (SB), variável 2114 que apresentou sinal filogenético na hipótese filogenética apresentada.

\begin{tabular}{|c|c|c|c|c|c|c|c|c|c|c|}
\hline Acrônimo & Ordem & Família & Espécie & B05 & B23 & B30 & SB & m & $\mathrm{pH}$ & Classe \\
\hline Viro_sebi & Magnoliales & Myristicaceae & Virola sebifera Aubl. & 0.37 & 0.71 & 0.21 & 0.50 & 0.82 & 0.28 & $\mathrm{D}$ \\
\hline Xylo_seri & Magnoliales & Annonaceae & Xylopia sericea A.St.-Hil. & 0.03 & 0.75 & 0.24 & 0.03 & 0.78 & 0.17 & $A$ \\
\hline Xylo_arom & Magnoliales & Annonaceae & Xylopia aromatica (Lam.) Mart. & 0.49 & 0.52 & 0.39 & 0.47 & 0.65 & 0.32 & D \\
\hline Unon_guat & Magnoliales & Annonaceae & Unonopsis guatterioides (A.DC.) R.E.Fr. & 0.21 & 0.75 & 0.15 & 0.04 & 0.99 & 0.91 & A \\
\hline Oxan_sess & Magnoliales & Annonaceae & Oxandra sessiliflora R.E.Fr. & 0.96 & 0.03 & 0.75 & 0.23 & 0.20 & 0.06 & C \\
\hline Card_calo & Magnoliales & Annonaceae & Cardiopetalum calophyllum Schltdl. & 0.38 & 0.62 & 0.23 & 0.26 & 0.91 & 0.65 & C \\
\hline Anno_dioi & Magnoliales & Annonaceae & Annona dioica A.St.-Hil. & 0.29 & 0.96 & 0.09 & 0.81 & 0.28 & 0.05 & G \\
\hline Anno_cras & Magnoliales & Annonaceae & Annona crassiflora Mart. & 0.28 & 0.69 & 0.56 & 0.21 & 0.67 & 0.22 & C \\
\hline Anno_cori & Magnoliales & Annonaceae & Annona coriacea Mart. & 0.64 & 0.36 & 0.63 & 0.55 & 0.34 & 0.46 & E \\
\hline Sipa_guia & Laurales & Siparunaceae & Siparuna guianensis Aubl. & 0.52 & 0.52 & 0.32 & 0.41 & 0.69 & 0.31 & D \\
\hline Mezi_cras & Laurales & Lauraceae & Mezilaurus crassiramea (Meisn.) Taub. & 0.83 & 0.44 & 0.70 & 0.71 & 0.27 & 0.18 & $\mathrm{~F}$ \\
\hline Ocot_pulc & Laurales & Lauraceae & Ocotea pulchella (Nees \& Mart.) Mez & 0.17 & 0.84 & 0.13 & 0.21 & 0.98 & 0.60 & C \\
\hline Ocot_mina & Laurales & Lauraceae & Ocotea minarum (Nees \& Mart.) Mez & 0.04 & 0.87 & 0.10 & 0.47 & 0.90 & 0.92 & D \\
\hline Ocot_cory & Laurales & Lauraceae & Ocotea corymbosa (Meisn.) Mez & 0.14 & 0.92 & 0.08 & 0.55 & 0.90 & 0.29 & E \\
\hline Eupl_inae & Proteales & Proteaceae & Euplassa inaequalis (Pohl) Engl. & 0.85 & 0.23 & 0.92 & 0.80 & 0.08 & 0.66 & G \\
\hline Roup_mont & Proteales & Proteaceae & Roupala montana Aubl. & 0.41 & 0.54 & 0.48 & 0.68 & 0.60 & 0.41 & $\mathrm{~F}$ \\
\hline Rham_elae & Rosales & Rhamnaceae & Rhamnidium elaeocarpum Reissek & 0.81 & 0.46 & 0.42 & 0.95 & 0.08 & 0.99 & H \\
\hline Ficu_enor & Rosales & Moraceae & Ficus enormis Mart. ex Miq. & 0.22 & 0.75 & 0.15 & 0.72 & 0.86 & 0.76 & $\mathrm{~F}$ \\
\hline Bros_gaud & Rosales & Moraceae & Brosimum gaudichaudii Trécul & 0.42 & 0.55 & 0.65 & 0.59 & 0.52 & 0.70 & $\mathrm{E}$ \\
\hline Cecr_pach & Rosales & Urticaceae & Cecropia pachystachya Trécul & 0.66 & 0.21 & 0.46 & 0.54 & 0.48 & 0.41 & $\mathrm{E}$ \\
\hline
\end{tabular}




\begin{tabular}{|c|c|c|c|c|c|c|c|c|c|c|}
\hline Acrônimo & Ordem & Família & Espécie & B05 & B23 & B30 & SB & $\mathbf{m}$ & $\mathrm{pH}$ & Classe \\
\hline Bauh_brev & Fabales & Fabaceae & Bauhinia brevipes Vogel & 0.02 & 0.19 & 0.83 & 0.97 & 0.04 & 0.95 & $\mathrm{H}$ \\
\hline Bauh_pulc & Fabales & Fabaceae & Bauhinia pulchella Benth. & 0.15 & 0.18 & 0.96 & 0.83 & 0.20 & 0.91 & G \\
\hline Bauh_rufa & Fabales & Fabaceae & Bauhinia rufa (Bong.) Steud. & 0.25 & 0.33 & 0.44 & 0.92 & 0.32 & 0.78 & $\mathrm{H}$ \\
\hline Copa_lang & Fabales & Fabaceae & Copaifera langsdorffii Desf. & 0.29 & 0.68 & 0.43 & 0.64 & 0.54 & 0.36 & $\mathrm{E}$ \\
\hline Copa_mart & Fabales & Fabaceae & Copaifera martii Hayne & 0.83 & 0.23 & 0.92 & 0.66 & 0.14 & 0.79 & $\mathrm{~F}$ \\
\hline Hyme_cour & Fabales & Fabaceae & Hymenaea courbaril L. & 0.88 & 0.18 & 0.35 & 0.84 & 0.09 & 0.55 & G \\
\hline Hyme_erio & Fabales & Fabaceae & Hymenaea eriogyne Benth. & 0.98 & 0.03 & 0.86 & 0.47 & 0.63 & 0.35 & D \\
\hline Hyme_mara & Fabales & Fabaceae & Hymenaea maranhensis Y.T.Lee \& Langenh. & 0.93 & 0.08 & 0.43 & 0.59 & 0.04 & 0.05 & $\mathrm{E}$ \\
\hline Hyme_mart & Fabales & Fabaceae & Hymenaea martiana Hayne & 0.30 & 0.91 & 0.28 & 0.13 & 0.87 & 0.10 & B \\
\hline Hyme_stig & Fabales & Fabaceae & Hymenaea stigonocarpa Mart. ex Hayne & 0.57 & 0.57 & 0.60 & 0.64 & 0.50 & 0.58 & $\mathrm{~F}$ \\
\hline Pelt_conf & Fabales & Fabaceae & Peltogyne confertiflora (Mart. ex Hayne) Benth. & 0.09 & 0.27 & 0.55 & 0.50 & 0.34 & 0.21 & $\mathrm{E}$ \\
\hline Mart_medi & Fabales & Fabaceae & Martiodendron mediterraneum (Mart. ex Benth.) R.C.Koeppen & 0.77 & 0.06 & 0.29 & 0.74 & 0.17 & 0.04 & $\mathrm{~F}$ \\
\hline Apul_leio & Fabales & Fabaceae & Apuleia leiocarpa (Vogel) J.F.Macbr. & 0.60 & 0.30 & 0.36 & 0.02 & 0.71 & 0.28 & A \\
\hline Dipt_aura & Fabales & Fabaceae & Diptychandra aurantiaca Tul. & 0.04 & 0.93 & 0.05 & 0.62 & 0.74 & 0.23 & $\mathrm{E}$ \\
\hline Cham_orbi & Fabales & Fabaceae & Chamaecrista orbiculata (Benth.) H.S.Irwin \& Barneby & 0.01 & 0.68 & 0.73 & 0.10 & 0.45 & 0.15 & A \\
\hline Senn_silv & Fabales & Fabaceae & Senna silvestris (Vell.) H.S.Irwin \& Barneby & 0.82 & 0.20 & 0.41 & 0.99 & 0.05 & 0.85 & $\mathrm{H}$ \\
\hline Senn_velu & Fabales & Fabaceae & Senna velutina (Vogel) H.S.Irwin \& Barneby & 0.84 & 0.90 & 0.59 & 0.14 & 0.53 & 0.47 & B \\
\hline Senn_rugo & Fabales & Fabaceae & Senna rugosa (G.Don) H.S.Irwin \& Barneby & 0.09 & 0.98 & 0.02 & 0.51 & 0.95 & 0.00 & $\mathrm{E}$ \\
\hline Ceno_macr & Fabales & Fabaceae & Cenostigma macrophyllum Tul. & 0.97 & 0.05 & 0.89 & 0.14 & 0.35 & 0.81 & B \\
\hline Pter_nite & Fabales & Fabaceae & Pterogyne nitens Tul. & 0.71 & 0.78 & 0.78 & 0.97 & 0.01 & 0.52 & $\mathrm{H}$ \\
\hline Tach_aure & Fabales & Fabaceae & Tachigali aurea Tul. & 0.51 & 0.47 & 0.69 & 0.63 & 0.53 & 0.75 & $\mathrm{E}$ \\
\hline Tach_subv & Fabales & Fabaceae & Tachigali subvelutina (Benth.) Oliveira-Filho & 0.53 & 0.35 & 0.73 & 0.11 & 0.59 & 0.42 & B \\
\hline Tach_vulg & Fabales & Fabaceae & Tachigali vulgaris L.G.Silva \& H.C.Lima & 0.81 & 0.12 & 0.28 & 0.31 & 0.36 & 0.03 & D \\
\hline Dimo_gard & Fabales & Fabaceae & Dimorphandra gardneriana Tul. & 0.86 & 0.41 & 0.59 & 0.25 & 0.67 & 0.13 & $\mathrm{C}$ \\
\hline Dimo_moll & Fabales & Fabaceae & Dimorphandra mollis Benth. & 0.55 & 0.50 & 0.72 & 0.42 & 0.55 & 0.50 & D \\
\hline Anad_colu & Fabales & Fabaceae & Anadenanthera colubrina (Vell.) Brenan & 0.88 & 0.32 & 0.34 & 0.90 & 0.14 & 0.89 & $\mathrm{H}$ \\
\hline Anad_pere & Fabales & Fabaceae & Anadenanthera peregrina (L.) Speg. & 0.06 & 0.87 & 0.07 & 0.74 & 0.68 & 0.13 & $\mathrm{~F}$ \\
\hline Plat_reti & Fabales & Fabaceae & Plathymenia reticulata Benth. & 0.35 & 0.43 & 0.14 & 0.67 & 0.61 & 0.63 & $\mathrm{~F}$ \\
\hline Park_plat & Fabales & Fabaceae & Parkia platycephala Benth. & 0.94 & 0.11 & 0.78 & 0.41 & 0.12 & 0.33 & D \\
\hline
\end{tabular}




\begin{tabular}{|c|c|c|c|c|c|c|c|c|c|c|}
\hline Acrônimo & Ordem & Família & Espécie & B05 & B23 & B30 & SB & m & $\mathrm{pH}$ & Classe \\
\hline Stry_cori & Fabales & Fabaceae & Stryphnodendron coriaceum Benth. & 0.20 & 0.09 & 0.18 & 0.18 & 0.38 & 0.78 & B \\
\hline Stry_rotu & Fabales & Fabaceae & Stryphnodendron rotundifolium Mart. & 0.70 & 0.32 & 0.58 & 0.32 & 0.37 & 0.34 & D \\
\hline Stry_adst & Fabales & Fabaceae & Stryphnodendron adstringens (Mart.) Coville & 0.91 & 0.80 & 0.68 & 0.08 & 0.94 & 0.25 & A \\
\hline Stry_poly & Fabales & Fabaceae & Stryphnodendron polyphyllum Mart. & 0.50 & 0.75 & 0.70 & 0.17 & 0.97 & 0.68 & B \\
\hline Mimo_seri & Fabales & Fabaceae & Mimosa sericantha Benth. & 0.54 & 0.85 & 0.09 & 0.05 & 0.22 & 0.18 & A \\
\hline Mimo_clau & Fabales & Fabaceae & Mimosa claussenii Benth. & 0.03 & 0.12 & 0.87 & 0.29 & 0.22 & 0.82 & C \\
\hline Sene_poly & Fabales & Fabaceae & Senegalia polyphylla (DC.) Britton \& Rose & 0.36 & 0.75 & 0.15 & 0.28 & 0.70 & 0.03 & C \\
\hline Ente_cont & Fabales & Fabaceae & Enterolobium contortisiliquum (Vell.) Morong & 0.02 & 0.07 & 0.47 & 0.19 & 0.24 & 0.19 & B \\
\hline Ente_gumm & Fabales & Fabaceae & Enterolobium gummiferum (Mart.) J.F.Macbr. & 0.47 & 0.61 & 0.76 & 0.21 & 0.60 & 0.40 & C \\
\hline Albi_niop & Fabales & Fabaceae & Albizia niopoides (Spruce ex Benth.) Burkart & 0.72 & 0.27 & 0.98 & 0.08 & 0.41 & 0.37 & A \\
\hline Swar_apet & Fabales & Fabaceae & Swartzia apetala Raddi & 0.65 & 0.58 & 0.05 & 0.37 & 0.10 & 0.34 & D \\
\hline Dipt_alat & Fabales & Fabaceae & Dipteryx alata Vogel & 0.36 & 0.34 & 0.38 & 0.86 & 0.27 & 0.57 & G \\
\hline Pter_pube & Fabales & Fabaceae & Pterodon pubescens (Benth.) Benth. & 0.96 & 0.56 & 0.06 & 0.38 & 0.66 & 0.84 & D \\
\hline Pter_emar & Fabales & Fabaceae & Pterodon emarginatus Vogel & 0.41 & 0.46 & 0.97 & 0.33 & 0.26 & 0.77 & D \\
\hline Luet_prae & Fabales & Fabaceae & Luetzelburgia praecox (Harms) Harms & 0.79 & 0.70 & 0.40 & 0.87 & 0.58 & 0.16 & G \\
\hline Cent_tome & Fabales & Fabaceae & Centrolobium tomentosum Guillem. ex Benth. & 0.07 & 0.97 & 0.00 & 0.88 & 0.55 & 0.14 & H \\
\hline Plat_eleg & Fabales & Fabaceae & Platypodium elegans Vogel & 0.68 & 0.75 & 0.38 & 0.84 & 0.77 & 0.36 & G \\
\hline Mach_acut & Fabales & Fabaceae & Machaerium acutifolium Vogel & 0.62 & 0.40 & 0.57 & 0.51 & 0.51 & 0.48 & E \\
\hline Mach_hirt & Fabales & Fabaceae & Machaerium hirtum (Vell.) Stellfeld & 0.88 & 0.21 & 0.90 & 0.51 & 0.06 & 0.50 & $\mathrm{E}$ \\
\hline Mach_opac & Fabales & Fabaceae & Machaerium opacum Vogel & 0.44 & 0.66 & 0.84 & 0.45 & 0.69 & 0.67 & D \\
\hline Dalb_misc & Fabales & Fabaceae & Dalbergia miscolobium Benth. & 0.50 & 0.61 & 0.35 & 0.58 & 0.73 & 0.30 & E \\
\hline Andi_cord & Fabales & Fabaceae & Andira cordata Arroyo ex R.T.Penn. \& H.C.Lima & 0.94 & 0.09 & 0.93 & 0.10 & 0.40 & 0.39 & A \\
\hline Andi_cuja & Fabales & Fabaceae & Andira cujabensis Benth. & 0.42 & 0.23 & 0.60 & 0.75 & 0.32 & 0.56 & G \\
\hline Andi_verm & Fabales & Fabaceae & Andira vermifuga (Mart.) Benth. & 0.73 & 0.49 & 0.82 & 0.18 & 0.59 & 0.64 & B \\
\hline Vata_macr & Fabales & Fabaceae & Vatairea macrocarpa (Benth.) Ducke & 0.64 & 0.44 & 0.77 & 0.45 & 0.46 & 0.49 & D \\
\hline Bowd_virg & Fabales & Fabaceae & Bowdichia virgilioides Kunth & 0.55 & 0.54 & 0.53 & 0.49 & 0.63 & 0.41 & D \\
\hline Lept_eleg & Fabales & Fabaceae & Leptolobium elegans Vogel & 0.28 & 0.71 & 0.19 & 0.17 & 0.91 & 0.58 & B \\
\hline Lept_dasy & Fabales & Fabaceae & Leptolobium dasycarpum Vogel & 0.45 & 0.53 & 0.75 & 0.60 & 0.65 & 0.61 & $E$ \\
\hline Conn_sube & Oxalidales & Connaraceae & Connarus suberosus Planch. & 0.45 & 0.53 & 0.75 & 0.34 & 0.62 & 0.46 & D \\
\hline
\end{tabular}




\begin{tabular}{|c|c|c|c|c|c|c|c|c|c|c|}
\hline Acrônimo & Ordem & Família & Espécie & B05 & B23 & B30 & SB & m & $\mathrm{pH}$ & Classe \\
\hline Rour_indu & Oxalidales & Connaraceae & Rourea induta Planch. & 0.36 & 0.25 & 0.72 & 0.37 & 0.57 & 0.64 & D \\
\hline Plen_popu & Celastrales & Celastraceae & Plenckia populnea Reissek & 0.51 & 0.70 & 0.53 & 0.19 & 0.86 & 0.30 & B \\
\hline Sala_elli & Celastrales & Celastraceae & Salacia elliptica (Mart. ex Schult.) G.Don & 0.86 & 0.45 & 0.47 & 0.44 & 0.66 & 0.55 & D \\
\hline Sala_cras & Celastrales & Celastraceae & Salacia crassifolia (Mart. ex Schult.) G.Don & 0.62 & 0.31 & 0.85 & 0.30 & 0.24 & 0.54 & C \\
\hline Case_arbo & Malpighiales & Salicaceae & Casearia arborea (Rich.) Urb. & 0.65 & 0.60 & 0.18 & 0.32 & 0.64 & 0.13 & D \\
\hline Case_gran & Malpighiales & Salicaceae & Casearia grandiflora Cambess. & 0.66 & 0.14 & 0.35 & 0.22 & 0.35 & 0.22 & C \\
\hline Case_rupe & Malpighiales & Salicaceae & Casearia rupestris Eichler & 0.37 & 0.39 & 0.49 & 1.00 & 0.02 & 1.00 & H \\
\hline Case_sylv & Malpighiales & Salicaceae & Casearia sylvestris Sw. & 0.43 & 0.50 & 0.48 & 0.73 & 0.36 & 0.66 & $\mathrm{~F}$ \\
\hline Cary_cune & Malpighiales & Caryocaraceae & Caryocar cuneatum Wittm. & 0.93 & 0.10 & 0.88 & 0.35 & 0.12 & 0.83 & D \\
\hline Cary_cori & Malpighiales & Caryocaraceae & Caryocar coriaceum Wittm. & 0.80 & 0.65 & 0.82 & 0.07 & 0.85 & 0.16 & A \\
\hline Cary_bras & Malpighiales & Caryocaraceae & Caryocar brasiliense Cambess. & 0.27 & 0.65 & 0.63 & 0.56 & 0.58 & 0.48 & E \\
\hline Pera_glab & Malpighiales & Peraceae & Pera glabrata (Schott) Poepp. ex Baill. & 0.24 & 0.79 & 0.20 & 0.65 & 0.93 & 0.40 & $\mathrm{~F}$ \\
\hline Laci_hass & Malpighiales & Lacistemataceae & Lacistema hasslerianum Chodat & 0.16 & 0.82 & 0.25 & 0.07 & 0.88 & 0.14 & A \\
\hline Alch_disc & Malpighiales & Euphorbiaceae & Alchornea discolor Poepp. & 0.99 & 0.10 & 0.39 & 0.33 & 0.09 & 0.31 & D \\
\hline Crot_flor & Malpighiales & Euphorbiaceae & Croton floribundus Spreng. & 0.26 & 0.84 & 0.07 & 0.76 & 0.45 & 0.16 & G \\
\hline Mabe_fist & Malpighiales & Euphorbiaceae & Mabea fistulifera Mart. & 0.71 & 0.48 & 0.20 & 0.76 & 0.20 & 0.09 & G \\
\hline Mabe_pohl & Malpighiales & Euphorbiaceae & Mabea pohliana (Benth.) Müll.Arg. & 0.77 & 0.06 & 0.29 & 0.74 & 0.17 & 0.04 & $\mathrm{~F}$ \\
\hline Mapr_guia & Malpighiales & Euphorbiaceae & Maprounea guianensis Aubl. & 0.18 & 0.55 & 0.38 & 0.16 & 0.63 & 0.20 & B \\
\hline Eryt_vacc & Malpighiales & Erythroxylaceae & Erythroxylum vacciniifolium Mart. & 0.86 & 0.48 & 0.81 & 0.92 & 0.02 & 0.97 & $\mathrm{H}$ \\
\hline Eryt_tort & Malpighiales & Erythroxylaceae & Erythroxylum tortuosum Mart. & 0.32 & 0.60 & 0.46 & 0.27 & 0.72 & 0.57 & C \\
\hline Eryt_sube & Malpighiales & Erythroxylaceae & Erythroxylum suberosum A.St.-Hil. & 0.35 & 0.52 & 0.54 & 0.61 & 0.67 & 0.53 & E \\
\hline Eryt_pell & Malpighiales & Erythroxylaceae & Erythroxylum pelleterianum A.St.-Hil. & 0.09 & 0.98 & 0.01 & 0.71 & 0.87 & 0.03 & $\mathrm{~F}$ \\
\hline Eryt_betu & Malpighiales & Erythroxylaceae & Erythroxylum betulaceum Mart. & 0.69 & 0.74 & 1.00 & 0.04 & 0.15 & 0.79 & A \\
\hline Eryt_daph & Malpighiales & Erythroxylaceae & Erythroxylum daphnites Mart. & 0.70 & 0.49 & 0.51 & 0.81 & 0.31 & 0.26 & G \\
\hline Eryt_deci & Malpighiales & Erythroxylaceae & Erythroxylum deciduum A.St.-Hil. & 0.48 & 0.57 & 0.68 & 0.15 & 0.73 & 0.72 & B \\
\hline Eryt_engl & Malpighiales & Erythroxylaceae & Erythroxylum engleri O.E.Schulz & 0.78 & 0.73 & 0.44 & 0.39 & 0.58 & 0.12 & D \\
\hline Clus_sell & Malpighiales & Clusiaceae & Clusia sellowiana Schltdl. & 0.19 & 0.93 & - & 0.27 & 0.26 & 0.08 & C \\
\hline Kiel_spec & Malpighiales & Calophyllaceae & Kielmeyera speciosa A.St.-Hil. & 0.78 & 0.32 & 0.80 & 0.49 & 0.80 & 0.20 & D \\
\hline Kiel_rubr & Malpighiales & Calophyllaceae & Kielmeyera rubriflora Cambess. & 0.13 & 0.16 & 0.62 & 0.53 & 0.46 & 0.25 & $\mathrm{E}$ \\
\hline
\end{tabular}




\begin{tabular}{|c|c|c|c|c|c|c|c|c|c|c|}
\hline Acrônimo & Ordem & Família & Espécie & B05 & B23 & B30 & SB & m & $\mathrm{pH}$ & Classe \\
\hline Kiel_lath & Malpighiales & Calophyllaceae & Kielmeyera lathrophyton Saddi & 0.54 & 0.38 & 0.96 & 0.06 & 0.68 & 0.48 & A \\
\hline Kiel_cori & Malpighiales & Calophyllaceae & Kielmeyera coriacea Mart. \& Zucc. & 0.40 & 0.59 & 0.64 & 0.48 & 0.56 & 0.68 & D \\
\hline Hete_byrs & Malpighiales & Malpighiaceae & Heteropterys byrsonimiifolia A.Juss. & 0.81 & 0.19 & 0.74 & 0.70 & 0.30 & 0.47 & $\mathrm{~F}$ \\
\hline Bani_lati & Malpighiales & Malpighiaceae & Banisteriopsis latifolia (A.Juss.) B.Gates & 0.74 & 0.11 & 0.86 & 0.69 & 0.25 & 0.71 & $\mathrm{~F}$ \\
\hline Byrs_seri & Malpighiales & Malpighiaceae & Byrsonima sericea DC. & 0.59 & 0.59 & 0.07 & 0.27 & 0.16 & 0.09 & c \\
\hline Byrs_verb & Malpighiales & Malpighiaceae & Byrsonima verbascifolia (L.) DC. & 0.20 & 0.63 & 0.57 & 0.20 & 0.78 & 0.60 & B \\
\hline Byrs_basi & Malpighiales & Malpighiaceae & Byrsonima basiloba A.Juss. & 0.56 & 0.69 & 0.43 & 0.06 & 0.84 & 0.65 & A \\
\hline Byrs_cocc & Malpighiales & Malpighiaceae & Byrsonima coccolobifolia Kunth & 0.40 & 0.56 & 0.56 & 0.33 & 0.71 & 0.44 & D \\
\hline Byrs_corr & Malpighiales & Malpighiaceae & Byrsonima correifolia A.Juss. & 0.67 & 0.74 & 0.93 & 0.10 & 0.21 & 0.27 & A \\
\hline Byrs_cras & Malpighiales & Malpighiaceae & Byrsonima crassifolia (L.) Kunth & 0.79 & 0.22 & 0.33 & 0.76 & 0.25 & 0.24 & G \\
\hline Byrs_cydo & Malpighiales & Malpighiaceae & Byrsonima cydoniifolia A.Juss. & 0.39 & 0.66 & 0.37 & 0.36 & 0.70 & 0.06 & D \\
\hline Byrs_inte & Malpighiales & Malpighiaceae & Byrsonima intermedia A.Juss. & 0.56 & 0.86 & 0.55 & 0.23 & 0.43 & 0.21 & C \\
\hline Byrs_pach & Malpighiales & Malpighiaceae & Byrsonima pachyphylla A.Juss. & 0.57 & 0.40 & 0.86 & 0.40 & 0.49 & 0.72 & D \\
\hline Oura_spec & Malpighiales & Ochnaceae & Ouratea spectabilis (Mart.) Engl. & 0.33 & 0.65 & 0.46 & 0.20 & 0.44 & 0.27 & B \\
\hline Oura_hexa & Malpighiales & Ochnaceae & Ouratea hexasperma (A.St.-Hil.) Baill. & 0.63 & 0.39 & 0.80 & 0.29 & 0.61 & 0.65 & C \\
\hline Oura_cast & Malpighiales & Ochnaceae & Ouratea castaneifolia (DC.) Engl. & 0.53 & 0.64 & 0.34 & 0.85 & 0.75 & 0.77 & G \\
\hline Tapu_amaz & Malpighiales & Dichapetalaceae & Tapura amazonica Poepp. \& Endl. & 0.90 & 0.02 & 0.85 & 0.09 & 0.16 & 0.92 & A \\
\hline Hirt_grac & Malpighiales & Chrysobalanaceae & Hirtella gracilipes (Hook.f.) Prance & 0.46 & 0.81 & 0.50 & 0.41 & 0.89 & 0.51 & D \\
\hline Hirt_glan & Malpighiales & Chrysobalanaceae & Hirtella glandulosa Spreng. & 0.69 & 0.42 & 0.52 & 0.46 & 0.45 & 0.42 & D \\
\hline Hirt_cili & Malpighiales & Chrysobalanaceae & Hirtella ciliata Mart. \& Zucc. & 0.89 & 0.17 & 0.61 & 0.12 & 0.39 & 0.24 & B \\
\hline Lica_gard & Malpighiales & Chrysobalanaceae & Licania gardneri (Hook.f.) Fritsch & 0.89 & 0.01 & 0.69 & 0.94 & 0.02 & 0.38 & $\mathrm{H}$ \\
\hline Lica_humi & Malpighiales & Chrysobalanaceae & Licania humilis Cham. \& Schltdl. & 0.07 & 0.91 & 0.12 & 0.11 & 0.95 & 0.22 & A \\
\hline Lica_scle & Malpighiales & Chrysobalanaceae & Licania sclerophylla (Hook.f.) Fritsch & 1.00 & 0.00 & 0.84 & 0.99 & - & 1.00 & $\mathrm{H}$ \\
\hline Coue_gran & Malpighiales & Chrysobalanaceae & Couepia grandiflora (Mart. \& Zucc.) Benth. & 0.45 & 0.63 & 0.80 & 0.54 & 0.66 & 0.59 & E \\
\hline Coch_viti & Malvales & Bixaceae & Cochlospermum vitifolium (Willd.) Spreng. & 0.80 & 0.15 & 0.81 & 0.97 & 0.01 & 0.93 & H \\
\hline Ster_stri & Malvales & Malvaceae & Sterculia striata A.St.-Hil. \& Naudin & 0.99 & 0.21 & 0.37 & 0.67 & 0.05 & 0.96 & $\mathrm{~F}$ \\
\hline Pseu_long & Malvales & Malvaceae & Pseudobombax longiflorum (Mart. \& Zucc.) A.Robyns & 0.65 & 0.22 & 0.66 & 0.86 & 0.25 & 0.85 & G \\
\hline Pseu_marg & Malvales & Malvaceae & Pseudobombax marginatum (A.St.-Hil.) A. Robyns & 0.06 & 0.20 & 0.92 & 0.94 & 0.10 & 0.93 & H \\
\hline Pseu_tome & Malvales & Malvaceae & Pseudobombax tomentosum (Mart. \& Zucc.) A.Robyns & 0.82 & 0.36 & 0.41 & 0.90 & 0.28 & 0.88 & $\mathrm{H}$ \\
\hline
\end{tabular}




\begin{tabular}{|c|c|c|c|c|c|c|c|c|c|c|}
\hline Acrônimo & Ordem & Família & Espécie & B05 & B23 & B30 & SB & m & $\mathrm{pH}$ & Classe \\
\hline Lueh_diva & Malvales & Malvaceae & Luehea divaricata Mart. \& Zucc. & 0.26 & 0.83 & 0.08 & 0.99 & 0.59 & 0.86 & $\mathrm{H}$ \\
\hline Lueh_pani & Malvales & Malvaceae & Luehea paniculata Mart. \& Zucc. & 0.73 & 0.18 & 0.54 & 0.82 & 0.13 & 0.90 & G \\
\hline Lueh_gran & Malvales & Malvaceae & Luehea grandiflora Mart. \& Zucc. & 0.18 & 0.90 & 0.11 & 0.70 & 0.72 & 0.30 & $\mathrm{~F}$ \\
\hline Guaz_ulmi & Malvales & Malvaceae & Guazuma ulmifolia Lam. & 0.85 & 0.16 & 0.61 & 0.83 & 0.08 & 0.94 & G \\
\hline Erio_grac & Malvales & Malvaceae & Eriotheca gracilipes (K.Schum.) A.Robyns & 0.52 & 0.40 & 0.71 & 0.36 & 0.64 & 0.45 & D \\
\hline Erio_pube & Malvales & Malvaceae & Eriotheca pubescens (Mart. \& Zucc.) Schott \& Endl. & 0.05 & 0.96 & 0.26 & 0.11 & 0.87 & 0.28 & A \\
\hline Erio_parv & Malvales & Malvaceae & Eriotheca parvifolia (Mart. \& Zucc.) A.Robyns & 0.99 & 0.01 & 0.88 & 0.26 & 0.37 & 0.80 & C \\
\hline Apei_tibo & Malvales & Malvaceae & Apeiba tibourbou Aubl. & 1.00 & 0.04 & 0.83 & 0.39 & 0.04 & 0.89 & D \\
\hline Anac_occi & Sapindales & Anacardiaceae & Anacardium occidentale L. & 0.76 & 0.19 & 0.91 & 0.35 & 0.33 & 0.55 & D \\
\hline Astr_frax & Sapindales & Anacardiaceae & Astronium fraxinifolium Schott & 0.60 & 0.43 & 0.70 & 0.77 & 0.37 & 0.75 & G \\
\hline Myra_urun & Sapindales & Anacardiaceae & Myracrodruon urundeuva Allemão & 0.15 & 0.45 & 0.49 & 0.95 & 0.07 & 0.97 & $\mathrm{H}$ \\
\hline Lith_moll & Sapindales & Anacardiaceae & Lithrea molleoides (Vell.) Engl. & 0.16 & 0.89 & 0.15 & 0.98 & 0.47 & 0.82 & H \\
\hline Tapi_guia & Sapindales & Anacardiaceae & Tapirira guianensis Aubl. & 0.12 & 0.68 & 0.25 & 0.68 & 0.75 & 0.38 & $\mathrm{~F}$ \\
\hline Tapi_obtu & Sapindales & Anacardiaceae & Tapirira obtusa (Benth.) J.D.Mitch. & 0.21 & 0.75 & 0.15 & 0.93 & 0.35 & 0.98 & $\mathrm{H}$ \\
\hline Tetr_alti & Sapindales & Burseraceae & Tetragastris altissima (Aubl.) Swart & 0.77 & 0.06 & 0.29 & 0.74 & 0.17 & 0.04 & $\mathrm{~F}$ \\
\hline Prot_ovat & Sapindales & Burseraceae & Protium ovatum Engl. & 0.07 & 0.81 & 0.99 & 0.05 & 0.16 & 0.62 & A \\
\hline Prot_hept & Sapindales & Burseraceae & Protium heptaphyllum (Aubl.) Marchand & 0.75 & 0.24 & 0.44 & 0.85 & 0.23 & 0.57 & G \\
\hline Cupa_vern & Sapindales & Sapindaceae & Cupania vernalis Cambess. & 0.13 & 0.88 & 0.08 & 0.80 & 0.79 & 0.95 & G \\
\hline Dilo_bipi & Sapindales & Sapindaceae & Dilodendron bipinnatum Radlk. & 0.32 & 0.29 & 0.71 & 0.93 & 0.17 & 0.95 & $\mathrm{H}$ \\
\hline Mago_pube & Sapindales & Sapindaceae & Magonia pubescens A.St.-Hil. & 0.72 & 0.28 & 0.67 & 0.85 & 0.19 & 0.77 & G \\
\hline Mata_guia & Sapindales & Sapindaceae & Matayba guianensis Aubl. & 0.38 & 0.30 & 0.34 & 0.69 & 0.61 & 0.74 & $\mathrm{~F}$ \\
\hline Sima_vers & Sapindales & Simaroubaceae & Simarouba versicolor A.St.-Hil. & 0.72 & 0.26 & 0.77 & 0.62 & 0.25 & 0.70 & E \\
\hline Sima_ferr & Sapindales & Simaroubaceae & Simaba ferruginea A.St.-Hil. & 0.77 & 0.72 & 1.00 & 0.07 & 0.14 & 0.63 & A \\
\hline Cabr_canj & Sapindales & Meliaceae & Cabralea canjerana (Vell.) Mart. & 0.08 & 0.95 & 0.04 & 0.56 & 0.83 & 0.15 & E \\
\hline Tric_pall & Sapindales & Meliaceae & Trichilia pallida Sw. & 0.13 & 0.88 & 0.14 & 0.95 & 0.68 & 0.82 & $\mathrm{H}$ \\
\hline Zant_rhoi & Sapindales & Rutaceae & Zanthoxylum rhoifolium Lam. & 0.49 & 0.33 & 0.40 & 0.84 & 0.12 & 0.33 & G \\
\hline Zant_ried & Sapindales & Rutaceae & Zanthoxylum riedelianum Engl. & 0.31 & 0.41 & 0.49 & 0.75 & 0.26 & 0.93 & G \\
\hline Zant_rigi & Sapindales & Rutaceae & Zanthoxylum rigidum Humb. \& Bonpl. exWilld. & 0.00 & 0.95 & 0.01 & 0.89 & 0.76 & 0.99 & H \\
\hline Buch_tetr & Myrtales & Combretaceae & Buchenavia tetraphylla (Aubl.) R.A.Howard & 0.61 & 0.62 & 0.04 & 0.05 & 0.24 & 0.07 & A \\
\hline
\end{tabular}




\begin{tabular}{|c|c|c|c|c|c|c|c|c|c|c|}
\hline Acrônimo & Ordem & Família & Espécie & B05 & B23 & B30 & SB & m & $\mathrm{pH}$ & Classe \\
\hline Buch_tome & Myrtales & Combretaceae & Buchenavia tomentosa Eichler & 0.34 & 0.42 & 0.61 & 0.83 & 0.34 & 0.86 & G \\
\hline Comb_mell & Myrtales & Combretaceae & Combretum mellifluum Eichler & 0.33 & 0.64 & 0.93 & 0.16 & 0.22 & 0.52 & B \\
\hline Term_phae & Myrtales & Combretaceae & Terminalia phaeocarpa Eichler & 0.64 & 0.35 & 0.32 & 0.64 & 0.39 & 0.84 & $\mathrm{~F}$ \\
\hline Term_glab & Myrtales & Combretaceae & Terminalia glabrescens Mart. & 0.25 & 0.90 & 0.13 & 0.35 & 0.92 & 0.38 & D \\
\hline Term_arge & Myrtales & Combretaceae & Terminalia argentea Mart. & 0.33 & 0.56 & 0.60 & 0.82 & 0.54 & 0.79 & G \\
\hline Term_fagi & Myrtales & Combretaceae & Terminalia fagifolia Mart. & 0.63 & 0.57 & 0.98 & 0.15 & 0.43 & 0.74 & B \\
\hline Lafo_vand & Myrtales & Lythraceae & Lafoensia vandelliana subsp. replicata (Pohl) Lourteig & 0.59 & 0.25 & 0.96 & 0.63 & 0.13 & 0.86 & $\mathrm{E}$ \\
\hline Lafo_paca & Myrtales & Lythraceae & Lafoensia pacari A.St.-Hil. & 0.34 & 0.83 & 0.26 & 0.48 & 0.82 & 0.21 & D \\
\hline Phys_scab & Myrtales & Lythraceae & Physocalymma scaberrimum Pohl & 0.90 & 0.07 & 0.75 & 0.79 & 0.06 & 0.53 & G \\
\hline Blep_sali & Myrtales & Myrtaceae & Blepharocalyx salicifolius (Kunth) O.Berg & - & 0.96 & 0.45 & 0.02 & 0.82 & 0.11 & A \\
\hline Camp_adam & Myrtales & Myrtaceae & Campomanesia adamantium (Cambess.) O.Berg & 0.09 & 0.98 & 0.02 & 0.89 & 0.42 & - & H \\
\hline Camp_velu & Myrtales & Myrtaceae & Campomanesia velutina (Cambess.) O.Berg & 0.24 & 0.69 & 0.31 & 0.98 & 0.77 & 0.25 & H \\
\hline Euge_puni & Myrtales & Myrtaceae & Eugenia punicifolia (Kunth) DC. & 0.27 & 0.87 & 0.10 & 0.18 & 0.90 & 0.12 & B \\
\hline Euge_dyse & Myrtales & Myrtaceae & Eugenia dysenterica DC. & 0.44 & 0.46 & 0.94 & 0.66 & 0.43 & 0.75 & $\mathrm{~F}$ \\
\hline Euge_aura & Myrtales & Myrtaceae & Eugenia aurata O.Berg & 0.09 & 0.72 & 0.20 & 0.31 & 0.78 & 0.19 & D \\
\hline Psid_pohl & Myrtales & Myrtaceae & Psidium pohlianum O.Berg & 0.22 & 0.75 & 0.15 & 0.01 & 0.98 & 0.23 & A \\
\hline Psid_myrt & Myrtales & Myrtaceae & Psidium myrtoides O.Berg & 0.51 & 0.41 & 0.97 & 0.24 & 0.41 & 0.80 & C \\
\hline Psid_laru & Myrtales & Myrtaceae & Psidium laruotteanum Cambess. & 0.60 & 0.94 & 0.99 & - & 0.99 & 0.46 & A \\
\hline Psid_salu & Myrtales & Myrtaceae & Psidium salutare (Kunth) O.Berg & 0.56 & 0.89 & 0.58 & 0.49 & 0.69 & 0.06 & D \\
\hline Myrc_lanu & Myrtales & Myrtaceae & Myrcia lanuginosa O.Berg & 0.92 & 0.14 & 0.71 & 0.94 & 0.01 & 0.51 & $\mathrm{H}$ \\
\hline Myrc_vari & Myrtales & Myrtaceae & Myrcia variabilis DC. & 0.20 & 0.75 & 0.18 & 0.15 & 0.94 & 0.80 & B \\
\hline Myrc_sple & Myrtales & Myrtaceae & Myrcia splendens (Sw.) DC. & 0.74 & 0.29 & 0.57 & 0.68 & 0.48 & 0.53 & $\mathrm{~F}$ \\
\hline Myrc_tome & Myrtales & Myrtaceae & Myrcia tomentosa (Aubl.) DC. & 0.32 & 0.70 & 0.27 & 0.77 & 0.83 & 0.31 & G \\
\hline Myrc_bell & Myrtales & Myrtaceae & Myrcia bella Cambess. & 0.16 & 0.97 & 0.11 & 0.50 & 0.89 & 0.05 & D \\
\hline Myrc_fenz & Myrtales & Myrtaceae & Myrcia fenzliana O.Berg & 0.61 & 0.94 & 0.98 & 0.00 & 0.99 & 0.69 & A \\
\hline Myrc_albo & Myrtales & Myrtaceae & Myrcia albotomentosa DC. & 0.62 & 0.51 & 0.45 & 0.71 & 0.30 & 0.50 & $\mathrm{~F}$ \\
\hline Myrc_sell & Myrtales & Myrtaceae & Myrcia selloi (Spreng.) N.Silveira & 0.82 & 0.07 & 0.29 & 0.62 & 0.11 & 0.08 & $\mathrm{E}$ \\
\hline Myrc_guia & Myrtales & Myrtaceae & Myrcia guianensis (Aubl.) DC. & 0.37 & 0.33 & 0.40 & 0.65 & 0.53 & 0.42 & $\mathrm{~F}$ \\
\hline Call_hass & Myrtales & Vochysiaceae & Callisthene hassleri Briq. & 0.28 & 0.10 & 0.87 & 0.61 & 0.29 & 0.90 & $\mathrm{E}$ \\
\hline
\end{tabular}




\begin{tabular}{|c|c|c|c|c|c|c|c|c|c|c|}
\hline Acrônimo & Ordem & Família & Espécie & B05 & B23 & B30 & SB & m & $\mathrm{pH}$ & Classe \\
\hline Call_majo & Myrtales & Vochysiaceae & Callisthene major Mart. \& Zucc. & 0.63 & 0.24 & 0.84 & 0.72 & 0.47 & 0.25 & $\mathrm{~F}$ \\
\hline Call_fasc & Myrtales & Vochysiaceae & Callisthene fasciculata Mart. & 0.70 & 0.22 & 0.58 & 0.89 & 0.15 & 0.83 & $\mathrm{H}$ \\
\hline Qual_dich & Myrtales & Vochysiaceae & Qualea dichotoma (Mart.) Warm. & 0.26 & 0.85 & 0.11 & 0.12 & 0.88 & 0.23 & B \\
\hline Qual_parv & Myrtales & Vochysiaceae & Qualea parviflora Mart. & 0.52 & 0.54 & 0.66 & 0.58 & 0.50 & 0.45 & $\mathrm{E}$ \\
\hline Qual_mult & Myrtales & Vochysiaceae & Qualea multiflora Mart. & 0.53 & 0.51 & 0.50 & 0.70 & 0.62 & 0.45 & $\mathrm{~F}$ \\
\hline Qual_cord & Myrtales & Vochysiaceae & Qualea cordata (Mart.) Spreng. & 0.23 & 0.95 & 0.14 & 0.22 & 0.84 & 0.08 & C \\
\hline Qual_gran & Myrtales & Vochysiaceae & Qualea grandiflora Mart. & 0.43 & 0.59 & 0.64 & 0.60 & 0.56 & 0.54 & $\mathrm{E}$ \\
\hline Salv_conv & Myrtales & Vochysiaceae & Salvertia convallariodora A.St.-Hil. & 0.39 & 0.38 & 0.76 & 0.30 & 0.52 & 0.61 & C \\
\hline Voch_cinn & Myrtales & Vochysiaceae & Vochysia cinnamomea Pohl & 0.92 & 0.09 & 0.63 & 0.20 & 0.32 & 0.43 & B \\
\hline Voch_tuca & Myrtales & Vochysiaceae & Vochysia tucanorum Mart. & 0.30 & 0.84 & 0.22 & 0.29 & 0.88 & 0.29 & c \\
\hline Voch_gard & Myrtales & Vochysiaceae & Vochysia gardneri Warm. & 0.41 & 0.55 & 0.97 & 0.09 & 0.29 & 0.37 & A \\
\hline Voch_thyr & Myrtales & Vochysiaceae & Vochysia thyrsoidea Pohl & 0.01 & 0.86 & 0.28 & 0.40 & 0.64 & 0.19 & D \\
\hline Voch_elli & Myrtales & Vochysiaceae & Vochysia elliptica Mart. & 0.04 & 0.66 & 0.91 & 0.03 & 0.85 & 0.58 & $A$ \\
\hline Voch_haen & Myrtales & Vochysiaceae & Vochysia haenkeana Mart. & 0.95 & 0.31 & 0.50 & 0.19 & 0.49 & 0.15 & B \\
\hline Voch_rufa & Myrtales & Vochysiaceae & Vochysia rufa Mart. & 0.59 & 0.62 & 0.55 & 0.37 & 0.81 & 0.33 & D \\
\hline Mico_albi & Myrtales & Melastomataceae & Miconia albicans (Sw.) Triana & 0.23 & 0.67 & 0.32 & 0.25 & 0.70 & 0.24 & C \\
\hline Mico_burc & Myrtales & Melastomataceae & Miconia burchellii Triana & 0.30 & 0.71 & 0.41 & 0.54 & 0.81 & 0.80 & E \\
\hline Mico_fall & Myrtales & Melastomataceae & Miconia fallax DC. & 0.43 & 0.74 & 0.51 & 0.04 & 0.79 & 0.70 & A \\
\hline Mico_ferr & Myrtales & Melastomataceae & Miconia ferruginata DC. & 0.75 & 0.28 & 0.89 & 0.03 & 0.71 & 0.27 & A \\
\hline Mico_leuc & Myrtales & Melastomataceae & Miconia leucocarpa DC. & 0.12 & 0.97 & 0.12 & 0.13 & 0.89 & 0.08 & B \\
\hline Mico_ligu & Myrtales & Melastomataceae & Miconia ligustroides (DC.) Naudin & 0.09 & 0.98 & 0.02 & 0.51 & 0.95 & 0.00 & E \\
\hline Mico_macr & Myrtales & Melastomataceae & Miconia macrothyrsa Benth. & 0.48 & 0.25 & 0.13 & 0.12 & 0.36 & 0.14 & B \\
\hline Mico_rubi & Myrtales & Melastomataceae & Miconia rubiginosa (Bonpl.) DC. & 0.09 & 0.98 & 0.02 & 0.51 & 0.95 & 0.00 & $\mathrm{E}$ \\
\hline Mico_sten & Myrtales & Melastomataceae & Miconia stenostachya DC. & 0.98 & 0.03 & 0.83 & 0.13 & 0.13 & 0.69 & B \\
\hline Mour_pusa & Myrtales & Melastomataceae & Mouriri pusa Gardner & 0.91 & 0.13 & 0.88 & 0.38 & 0.29 & 0.43 & D \\
\hline Mour_elli & Myrtales & Melastomataceae & Mouriri elliptica Mart. & 0.91 & 0.15 & 0.89 & 0.65 & 0.19 & 0.67 & $\mathrm{~F}$ \\
\hline Davi_elli & Dilleniales & Dilleniaceae & Davilla elliptica A.St.-Hil. & 0.58 & 0.36 & 0.77 & 0.43 & 0.44 & 0.60 & D \\
\hline Cura_amer & Dilleniales & Dilleniaceae & Curatella americana L. & 0.57 & 0.34 & 0.52 & 0.73 & 0.40 & 0.72 & $\mathrm{~F}$ \\
\hline Agon_bras & Santalales & Opiliaceae & Agonandra brasiliensis Miers ex Benth. \& Hook.f. & 0.35 & 0.49 & 0.69 & 0.56 & 0.47 & 0.73 & E \\
\hline
\end{tabular}




\begin{tabular}{|c|c|c|c|c|c|c|c|c|c|c|}
\hline Acrônimo & Ordem & Família & Espécie & B05 & B23 & B30 & SB & m & $\mathrm{pH}$ & Classe \\
\hline Heis_ovat & Santalales & Olacaceae & Heisteria ovata Benth. & 0.44 & 0.73 & 0.95 & 0.34 & 0.57 & 0.63 & D \\
\hline Xime_amer & Santalales & Olacaceae & Ximenia americana L. & 0.76 & 0.47 & 0.94 & 0.74 & 0.03 & 0.89 & $\mathrm{~F}$ \\
\hline Guap_grac & Caryophyllales & Nyctaginaceae & Guapira graciliflora (Mart. ex Schmidt) Lundell & 0.74 & 0.24 & 0.62 & 0.34 & 0.75 & 0.74 & D \\
\hline Guap_noxi & Caryophyllales & Nyctaginaceae & Guapira noxia (Netto) Lundell & 0.25 & 0.67 & 0.31 & 0.59 & 0.81 & 0.35 & $\mathrm{E}$ \\
\hline Guap_oppo & Caryophyllales & Nyctaginaceae & Guapira opposita (Vell.) Reitz & 0.01 & 0.92 & 0.05 & 0.48 & 0.86 & 0.11 & D \\
\hline Neea_thei & Caryophyllales & Nyctaginaceae & Neea theifera Oerst. & 0.34 & 0.37 & 0.68 & 0.57 & 0.56 & 0.71 & E \\
\hline Cocc_moll & Caryophyllales & Polygonaceae & Coccoloba mollis Casar. & 0.55 & 0.30 & 0.36 & 0.81 & 0.74 & 0.87 & G \\
\hline Nora_guia & Ericales & Marcgraviaceae & Norantea guianensis Aubl. & 0.75 & 0.94 & 0.29 & 0.44 & 0.79 & 0.12 & D \\
\hline Esch_nana & Ericales & Lecythidaceae & Eschweilera nana (O.Berg) Miers & 0.96 & 0.17 & 0.91 & 0.08 & 0.75 & 0.29 & A \\
\hline Pout_gard & Ericales & Sapotaceae & Pouteria gardneri (Mart. \& Miq.) Baehni & 0.16 & 0.82 & 0.25 & 1.00 & 0.94 & 0.94 & H \\
\hline Pout_rami & Ericales & Sapotaceae & Pouteria ramiflora (Mart.) Radlk. & 0.67 & 0.50 & 0.74 & 0.36 & 0.46 & 0.37 & D \\
\hline Pout_tort & Ericales & Sapotaceae & Pouteria torta (Mart.) Radlk. & 0.31 & 0.80 & 0.78 & 0.30 & 0.76 & 0.59 & C \\
\hline Chry_marg & Ericales & Sapotaceae & Chrysophyllum marginatum (Hook. \& Arn.) Radlk. & 0.03 & 0.91 & 0.06 & 0.87 & 0.48 & 0.96 & G \\
\hline Dios_cocc & Ericales & Ebenaceae & Diospyros coccolobifolia Mart. ex Miq. & 0.87 & 0.08 & 0.33 & 0.58 & 0.11 & 0.06 & E \\
\hline Dios_hisp & Ericales & Ebenaceae & Diospyros hispida A.DC. & 0.68 & 0.44 & 0.79 & 0.44 & 0.51 & 0.69 & D \\
\hline Dios_seri & Ericales & Ebenaceae & Diospyros sericea A.DC. & 0.73 & 0.11 & 0.48 & 0.78 & 0.33 & 0.44 & G \\
\hline Cybi_dete & Ericales & Primulaceae & Cybianthus detergens Mart. & 0.47 & 0.35 & 0.67 & 0.91 & 0.73 & 0.59 & H \\
\hline Myrs_umbe & Ericales & Primulaceae & Myrsine umbellata Mart. & 0.19 & 0.79 & 0.19 & 0.22 & 0.92 & 0.85 & C \\
\hline Myrs_guia & Ericales & Primulaceae & Myrsine guianensis (Aubl.) Kuntze & 0.05 & 0.73 & 0.10 & 0.25 & 0.83 & 0.17 & C \\
\hline Symp_plat & Ericales & Symplocaceae & Symplocos platyphylla (Pohl) Benth. & 0.21 & 0.75 & 0.15 & 0.02 & 0.98 & 0.91 & A \\
\hline Symp_nite & Ericales & Symplocaceae & Symplocos nitens (Pohl) Benth. & 0.17 & 0.82 & 0.23 & 0.86 & 0.95 & 0.92 & G \\
\hline Symp_pube & Ericales & Symplocaceae & Symplocos pubescens Klotzsch exBenth. & 0.21 & 0.75 & 0.15 & 0.01 & 1.00 & 0.94 & A \\
\hline Styr_camp & Ericales & Styracaceae & Styrax camporum Pohl & 0.19 & 0.80 & 0.23 & 0.73 & 0.93 & 0.47 & $\mathrm{~F}$ \\
\hline Styr_ferr & Ericales & Styracaceae & Styrax ferrugineus Nees \& Mart. & 0.25 & 0.79 & 0.24 & 0.17 & 0.92 & 0.39 & B \\
\hline Ferd_elli & Gentianales & Rubiaceae & Ferdinandusa elliptica (Pohl) Pohl & 0.89 & 0.05 & 0.76 & 0.67 & 0.27 & 0.66 & $\mathrm{~F}$ \\
\hline Cous_hydr & Gentianales & Rubiaceae & Coussarea hydrangeifolia (Benth.) Müll.Arg. & 0.67 & 0.29 & 0.42 & 0.61 & 0.65 & 0.44 & E \\
\hline Fara_hyac & Gentianales & Rubiaceae & Faramea hyacinthina Mart. & 0.21 & 0.75 & 0.15 & 0.91 & 0.60 & 0.56 & H \\
\hline Guet_vibu & Gentianales & Rubiaceae & Guettarda viburnoides Cham. \& Schltdl. & 0.80 & 0.25 & 0.54 & 0.93 & 0.23 & 0.90 & H \\
\hline Geni_amer & Gentianales & Rubiaceae & Genipa americana L. & 0.84 & 0.26 & 0.22 & 0.78 & 0.21 & 0.62 & G \\
\hline
\end{tabular}




\begin{tabular}{|c|c|c|c|c|c|c|c|c|c|c|}
\hline Acrônimo & Ordem & Família & Espécie & B05 & B23 & B30 & SB & m & $\mathrm{pH}$ & Classe \\
\hline Pali_rigi & Gentianales & Rubiaceae & Palicourea rigida Kunth & 0.29 & 0.37 & 0.42 & 0.23 & 0.74 & 0.43 & C \\
\hline Toco_form & Gentianales & Rubiaceae & Tocoyena formosa (Cham. \& Schltdl.) K.Schum. & 0.66 & 0.28 & 0.66 & 0.42 & 0.49 & 0.62 & D \\
\hline Rudg_vibu & Gentianales & Rubiaceae & Rudgea viburnoides (Cham.) Benth. & 0.24 & 0.67 & 0.27 & 0.77 & 0.80 & 0.54 & G \\
\hline Alib_edul & Gentianales & Rubiaceae & Alibertia edulis (Rich.) A.Rich. & 0.46 & 0.34 & 0.62 & 0.79 & 0.38 & 0.71 & G \\
\hline Amai_guia & Gentianales & Rubiaceae & Amaioua guianensis Aubl. & 0.09 & 0.98 & 0.02 & 0.51 & 0.95 & 0.00 & $\mathrm{E}$ \\
\hline Chom_ribe & Gentianales & Rubiaceae & Chomelia ribesioides Benth. ex A.Gray & 0.75 & - & 0.95 & 0.63 & 0.06 & 0.87 & E \\
\hline Chom_obtu & Gentianales & Rubiaceae & Chomelia obtusa Cham. \& Schltdl. & 0.06 & 0.48 & 0.95 & 0.39 & 0.38 & 0.84 & D \\
\hline Chom_pohl & Gentianales & Rubiaceae & Chomelia pohliana Müll.Arg. & 0.76 & 0.01 & 0.73 & 0.78 & 0.18 & 0.88 & G \\
\hline Cord_sess & Gentianales & Rubiaceae & Cordiera sessilis (Vell.) Kuntze & 0.97 & 0.81 & 0.82 & 0.42 & 0.85 & 0.34 & D \\
\hline Cord_elli & Gentianales & Rubiaceae & Cordiera elliptica (Cham.) Kuntze & 0.27 & 0.43 & 0.09 & 0.43 & 0.28 & 0.32 & D \\
\hline Cord_macr & Gentianales & Rubiaceae & Cordiera macrophylla (K.Schum.) Kuntze & 0.39 & 0.45 & 0.21 & 0.16 & 0.84 & 0.56 & B \\
\hline Stry_pseu & Gentianales & Loganiaceae & Strychnos pseudoquina A.St.-Hil. & 0.08 & 0.53 & 0.33 & 0.24 & 0.76 & 0.26 & C \\
\hline Anto_ovat & Gentianales & Loganiaceae & Antonia ovata Pohl & 0.87 & 0.14 & 0.36 & 0.31 & 0.31 & 0.35 & C \\
\hline Aspi_macr & Gentianales & Apocynaceae & Aspidosperma macrocarpon Mart. & 0.71 & 0.37 & 0.87 & 0.25 & 0.54 & 0.49 & C \\
\hline Aspi_mult & Gentianales & Apocynaceae & Aspidosperma multiflorum A.DC. & 0.84 & 0.16 & 0.45 & 0.79 & 0.30 & 0.49 & G \\
\hline Aspi_tome & Gentianales & Apocynaceae & Aspidosperma tomentosum Mart. & 0.46 & 0.51 & 0.90 & 0.57 & 0.52 & 0.68 & E \\
\hline Aspi_subi & Gentianales & Apocynaceae & Aspidosperma subincanum Mart. & 0.50 & 0.15 & 0.67 & 0.88 & 0.33 & 0.76 & H \\
\hline Aspi_aust & Gentianales & Apocynaceae & Aspidosperma australe Müll.Arg. & 0.97 & 0.13 & 0.81 & 0.98 & 0.00 & 0.96 & $\mathrm{H}$ \\
\hline Aspi_nobi & Gentianales & Apocynaceae & Aspidosperma nobile Müll.Arg. & 0.85 & 0.08 & 0.79 & 0.80 & 0.10 & 0.78 & G \\
\hline Hanc_spec & Gentianales & Apocynaceae & Hancornia speciosa Gomes & 0.61 & 0.39 & 0.64 & 0.28 & 0.51 & 0.32 & C \\
\hline Hima_obov & Gentianales & Apocynaceae & Himatanthus obovatus (Müll.Arg.) Woodson & 0.58 & 0.38 & 0.79 & 0.43 & 0.44 & 0.67 & D \\
\hline Hima_sucu & Gentianales & Apocynaceae & Himatanthus sucuuba (Spruce ex Müll.Arg.) Woodson & 0.90 & 0.05 & 0.47 & 0.32 & 0.18 & 0.10 & D \\
\hline Hima_arti & Gentianales & Apocynaceae & Himatanthus articulatus (Vahl) Woodson & 0.98 & 0.04 & 0.53 & 0.28 & 0.07 & 0.64 & C \\
\hline Aegi_vert & Lamiales & Lamiaceae & Aegiphila verticillata Vell. & 0.14 & 0.72 & 0.31 & 0.40 & 0.80 & 0.18 & D \\
\hline Hypt_canu & Lamiales & Lamiaceae & Hyptidendron canum (Pohl ex Benth.) Harley & 0.15 & 0.85 & 0.37 & 0.09 & 0.91 & 0.02 & A \\
\hline Vite_cymo & Lamiales & Lamiaceae & Vitex cymosa Bertero ex Spreng. & 0.95 & 0.31 & 0.21 & 0.55 & 0.05 & 0.99 & E \\
\hline Vite_poly & Lamiales & Lamiaceae & Vitex polygama Cham. & 0.93 & 0.04 & 0.51 & 0.91 & 0.03 & 0.10 & H \\
\hline Cybi_anti & Lamiales & Bignoniaceae & Cybistax antisyphilitica (Mart.) Mart. & 0.54 & 0.27 & 0.52 & 0.66 & 0.62 & 0.07 & $\mathrm{~F}$ \\
\hline Zeyh_mont & Lamiales & Bignoniaceae & Zeyheria montana Mart. & 0.40 & 0.60 & 0.90 & 0.82 & 0.31 & 0.81 & G \\
\hline
\end{tabular}




\begin{tabular}{|c|c|c|c|c|c|c|c|c|c|c|}
\hline Acrônimo & Ordem & Família & Espécie & B05 & B23 & B30 & SB & $\mathbf{m}$ & $\mathrm{pH}$ & Classe \\
\hline Jaca_bras & Lamiales & Bignoniaceae & Jacaranda brasiliana (Lam.) Pers. & 0.92 & 0.12 & 0.94 & 0.90 & 0.07 & 0.97 & $\mathrm{H}$ \\
\hline Jaca_cusp & Lamiales & Bignoniaceae & Jacaranda cuspidifolia Mart. & 0.94 & 0.61 & 0.12 & 0.96 & 0.03 & 0.83 & $\mathrm{H}$ \\
\hline Jaca_caro & Lamiales & Bignoniaceae & Jacaranda caroba (Vell.) DC. & 0.09 & 0.98 & 0.02 & 0.51 & 0.95 & 0.00 & $\mathrm{E}$ \\
\hline Tabe_rose & Lamiales & Bignoniaceae & Tabebuia roseoalba (Ridl.) Sandwith & 0.79 & 0.63 & 0.19 & 0.96 & 0.15 & 0.98 & $\mathrm{H}$ \\
\hline Tabe_aure & Lamiales & Bignoniaceae & Tabebuia aurea (Silva Manso) Benth. \& Hook.f. ex S.Moore & 0.58 & 0.47 & 0.59 & 0.69 & 0.50 & 0.76 & $\mathrm{~F}$ \\
\hline Hand_serr & Lamiales & Bignoniaceae & Handroanthus serratifolius (A.H.Gentry) S.Grose & 0.47 & 0.13 & 0.65 & 0.38 & 0.40 & 0.52 & $\mathrm{D}$ \\
\hline Hand_ochr & Lamiales & Bignoniaceae & Handroanthus ochraceus (Cham.) Mattos & 0.49 & 0.58 & 0.56 & 0.56 & 0.55 & 0.61 & $\mathrm{E}$ \\
\hline Hand_impe & Lamiales & Bignoniaceae & Handroanthus impetiginosus Mattos & 0.83 & 0.58 & 0.24 & 0.92 & 0.23 & 0.75 & $\mathrm{H}$ \\
\hline Sola_lyco & Solanales & Solanaceae & Solanum lycocarpum A.St.-Hil. & 0.38 & 0.92 & 0.22 & 0.45 & 0.41 & 0.11 & $\mathrm{D}$ \\
\hline Cord_supe & Boraginales & Boraginaceae & Cordia superba Cham. & 0.48 & 0.02 & 0.25 & 0.06 & 0.21 & 0.25 & $A$ \\
\hline Cord_sell & Boraginales & Boraginaceae & Cordia sellowiana Cham. & 0.69 & 0.42 & 0.65 & 0.72 & 0.42 & 0.88 & $\mathrm{~F}$ \\
\hline Cord_glab & Boraginales & Boraginaceae & Cordia glabrata (Mart.) A.DC. & 0.87 & 0.17 & 0.99 & 0.87 & 0.11 & 0.51 & G \\
\hline Emmo_nite & Boraginales & Icacinaceae & Emmotum nitens (Benth.) Miers & 0.68 & 0.26 & 0.72 & 0.46 & 0.39 & 0.39 & D \\
\hline Goch_pulc & Asterales & Asteraceae & Gochnatia pulchra Cabrera & 0.09 & 0.98 & 0.02 & 0.51 & 0.95 & 0.00 & $\mathrm{E}$ \\
\hline Pipt_rotu & Asterales & Asteraceae & Piptocarpha rotundifolia (Less.) Baker & 0.14 & 0.64 & 0.39 & 0.26 & 0.72 & 0.40 & C \\
\hline Erem_glom & Asterales & Asteraceae & Eremanthus glomeratus Less. & 0.31 & 0.86 & 0.30 & 0.01 & 1.00 & 0.73 & $A$ \\
\hline Vern_ferr & Asterales & Asteraceae & Vernonanthura ferruginea (Less.) H.Rob. & 0.42 & 0.20 & 0.85 & 0.60 & 0.42 & 0.87 & $\mathrm{E}$ \\
\hline Dend_cune & Apiales & Araliaceae & Dendropanax cuneatus (DC.) Decne. \& Planch. & 0.08 & 0.93 & 0.01 & 0.96 & 0.19 & 0.98 & $\mathrm{H}$ \\
\hline Sche_dist & Apiales & Araliaceae & Schefflera distractiflora (Harms) Frodin & 0.95 & 0.02 & 0.74 & 0.24 & 0.09 & 0.73 & C \\
\hline Sche_macr & Apiales & Araliaceae & Schefflera macrocarpa (Cham. \& Schltdl.) Frodin & 0.18 & 0.88 & 0.25 & 0.14 & 0.93 & 0.35 & B \\
\hline Sche_moro & Apiales & Araliaceae & Schefflera morototoni (Aubl.) Maguire et al. & 0.02 & 0.89 & 0.06 & 0.88 & 0.77 & 0.20 & $\mathrm{H}$ \\
\hline Sche_vino & Apiales & Araliaceae & Schefflera vinosa (Cham. \& Schltdl.) Frodin \& Fiaschi & 0.05 & 0.82 & 0.27 & 0.46 & 0.57 & 0.17 & D \\
\hline
\end{tabular}




\section{MATERIAL SUPLEMENTAR 2}

2119 Pontuação das espécies nos eixos PC1 e PC2 da análise de componentes principais dos valores de associação entre espécies arbóreas do Cerrado, 2120 variáveis edáficas e climáticas; e resultados significativos $(\alpha=0,05)$ das regressões lineares realizadas por espécie para todas as variáveis 2121 climáticas e edáficas selecionadas. PC1= componente principal $1 ; \mathrm{PC2}=$ componente principal $2 ; \mathrm{SB}=$ soma de bases $\left(\mathrm{cmolc} \mathrm{dm}{ }^{-1}\right) ; m=$ saturação por alumínio (\%); pH = acidez; Bio05 = temperatura máxima (ํ) ; Bio23 = sazonalidade da radiação (W m-2); Bio30 = menor índice de umidade.

\begin{tabular}{|c|c|c|c|c|c|c|c|c|c|c|c|c|c|c|c|c|}
\hline Família & Espécie & PC1 & PC2 & $\begin{array}{c}\text { SB } \\
F_{1,78} \\
\end{array}$ & $p$ & $\begin{array}{c}\mathbf{m} \\
\mathbf{F}_{1,78}\end{array}$ & $p$ & $\begin{array}{c}\mathrm{pH} \\
\mathrm{F}_{1,78}\end{array}$ & $p$ & $\begin{array}{c}\text { Bio05 } \\
F_{1,78} \\
\end{array}$ & $p$ & $\begin{array}{c}\text { Bio23 } \\
F_{1,78} \\
\end{array}$ & $p$ & $\begin{array}{c}\text { Bio30 } \\
F_{1,78} \\
\end{array}$ & $p$ & $\mathbf{N}$ \\
\hline Anacardiaceae & Anacardium occidentale L. & 1.52 & 0.87 & N.S. & N.S. & 32.58 & $<0.001$ & N.S. & N.S. & 23.91 & $<0.001$ & 14.36 & $<0.001$ & 15.35 & $<0.001$ & 44 \\
\hline Anacardiaceae & Astronium fraxinifolium Schott & 1.03 & -0.27 & 14.80 & $<0.001$ & 6.49 & 0.013 & 4.11 & 0.046 & 6.92 & 0.010 & N.S. & N.S. & N.S. & N.S. & 53 \\
\hline Anacardiaceae & Lithrea molleoides (Vell.) Engl. & -0.35 & -3.33 & 8.72 & 0.004 & N.S. & N.S. & N.S. & N.S. & 15.76 & $<0.001$ & N.S. & N.S. & N.S. & N.S. & 5 \\
\hline Anacardiaceae & Myracrodruon urundeuva Allemão & 1.35 & -2.57 & 33.26 & $<0.001$ & N.S. & N.S. & 13.25 & $<0.001$ & N.S. & N.S. & N.S. & N.S. & N.S. & N.S. & 16 \\
\hline Anacardiaceae & Tapirira guianensis Aubl. & -0.85 & -0.48 & N.S. & N.S. & 10.08 & 0.002 & N.S. & N.S. & 8.83 & 0.004 & N.S. & N.S. & N.S. & N.S. & 26 \\
\hline Anacardiaceae & Tapirira obtusa (Benth.) J.D.Mitch. & 0.09 & -2.27 & N.S. & N.S. & N.S. & N.S. & N.S. & N.S. & 4.04 & 0.047 & N.S. & N.S. & N.S. & N.S. & 2 \\
\hline Annonaceae & Annona coriacea Mart. & 0.82 & 0.26 & N.S. & N.S. & 9.65 & 0.003 & N.S. & N.S. & N.S. & N.S. & 4.90 & 0.029 & N.S. & N.S. & 43 \\
\hline Annonaceae & Annona crassiflora Mart. & -0.50 & 0.65 & 4.90 & 0.030 & N.S. & N.S. & 4.71 & 0.033 & 5.54 & 0.021 & N.S. & N.S. & N.S. & N.S. & 39 \\
\hline Annonaceae & Annona dioica A.St.-Hil. & -2.21 & -0.69 & N.S. & N.S. & N.S. & N.S. & 7.40 & 0.008 & 4.19 & 0.043 & N.S. & N.S. & N.S. & N.S. & 3 \\
\hline Annonaceae & Cardiopetalum calophyllum Schltdl. & -1.09 & 0.30 & N.S. & N.S. & 6.64 & 0.012 & N.S. & N.S. & N.S. & N.S. & N.S. & N.S. & N.S. & N.S. & 8 \\
\hline Annonaceae & Oxandra sessiliflora R.E.Fr. & 1.32 & 1.80 & N.S. & N.S. & N.S. & N.S. & N.S. & N.S. & N.S. & N.S. & N.S. & N.S. & N.S. & N.S. & 2 \\
\hline Annonaceae & Unonopsis guatterioides (A.DC.) R.E.Fr. & -2.10 & 0.50 & N.S. & N.S. & N.S. & N.S. & N.S. & N.S. & N.S. & N.S. & N.S. & N.S. & N.S. & N.S. & 2 \\
\hline Annonaceae & Xylopia aromatica (Lam.) Mart. & -0.09 & 0.34 & N.S. & N.S. & 6.22 & 0.015 & N.S. & N.S. & N.S. & N.S. & 16.00 & $<0.001$ & N.S. & N.S. & 49 \\
\hline Annonaceae & Xylopia sericea A.St.-Hil. & -2.50 & 0.36 & 3.98 & 0.049 & N.S. & N.S. & N.S. & N.S. & N.S. & N.S. & N.S. & N.S. & N.S. & N.S. & 4 \\
\hline Apocynaceae & Aspidosperma australe Müll.Arg. & 3.15 & -2.28 & 8.39 & 0.004 & N.S. & N.S. & N.S. & N.S. & N.S. & N.S. & N.S. & N.S. & N.S. & N.S. & 4 \\
\hline Apocynaceae & Aspidosperma macrocarpon Mart. & 0.85 & 0.93 & 5.08 & 0.026 & N.S. & N.S. & N.S. & N.S. & N.S. & N.S. & N.S. & N.S. & 4.02 & 0.048 & 30 \\
\hline Apocynaceae & Aspidosperma multiflorum A.DC. & 1.39 & -0.12 & N.S. & N.S. & N.S. & N.S. & N.S. & N.S. & N.S. & N.S. & N.S. & N.S. & N.S. & N.S. & 4 \\
\hline Apocynaceae & Aspidosperma nobile Müll.Arg. & 2.24 & -0.07 & 6.45 & 0.013 & N.S. & N.S. & N.S. & N.S. & N.S. & N.S. & N.S. & N.S. & N.S. & N.S. & 14 \\
\hline Apocynaceae & Aspidosperma subincanum Mart. & 1.48 & -0.66 & 6.92 & 0.010 & N.S. & N.S. & N.S. & N.S. & N.S. & N.S. & N.S. & N.S. & N.S. & N.S. & 21 \\
\hline Apocynaceae & Aspidosperma tomentosum Mart. & 0.78 & 0.34 & N.S. & N.S. & N.S. & N.S. & 4.95 & 0.029 & N.S. & N.S. & N.S. & N.S. & 18.25 & $<0.001$ & 46 \\
\hline Apocynaceae & Hancornia speciosa Gomes & 0.35 & 0.73 & N.S. & N.S. & N.S. & N.S. & 4.67 & 0.033 & N.S. & N.S. & N.S. & N.S. & N.S. & N.S. & 42 \\
\hline
\end{tabular}




\begin{tabular}{|c|c|c|c|c|c|c|c|c|c|c|c|c|c|c|c|c|}
\hline Família & Espécie & PC1 & PC2 & $\begin{array}{c}\text { SB } \\
\mathbf{F}_{1,78}\end{array}$ & $p$ & $\begin{array}{c}m \\
F_{1,78}\end{array}$ & $p$ & $\begin{array}{c}\mathrm{pH} \\
\mathrm{F}_{1,78}\end{array}$ & $p$ & $\begin{array}{c}\text { Bio05 } \\
F_{1,78} \\
\end{array}$ & $p$ & $\begin{array}{c}\text { Bio23 } \\
F_{1,78} \\
\end{array}$ & $p$ & $\begin{array}{c}\text { Bio30 } \\
F_{1,78} \\
\end{array}$ & $p$ & $\mathbf{N}$ \\
\hline Apocynaceae & Himatanthus articulatus (Vahl) Woodson & 2.08 & 0.86 & N.S. & N.S. & N.S. & N.S. & N.S. & N.S. & N.S. & N.S. & 11.33 & 0.001 & N.S. & N.S. & 3 \\
\hline Apocynaceae & $\begin{array}{l}\text { Himatanthus obovatus (Müll.Arg.) Woodson } \\
\text { Himatanthus sucuuba (Spruce ex Müll.Arg.) }\end{array}$ & 0.87 & 0.42 & N.S. & N.S. & 14.70 & $<0.001$ & N.S. & N.S. & N.S. & N.S. & N.S. & N.S. & N.S. & N.S. & 53 \\
\hline Apocynaceae & Woodson & 1.15 & 1.35 & N.S. & N.S. & N.S. & N.S. & 5.64 & 0.020 & N.S. & N.S. & 9.31 & 0.003 & N.S. & N.S. & 4 \\
\hline Araliaceae & Dendropanax cuneatus (DC.) Decne. \& Planch. & -1.01 & -4.05 & 4.48 & 0.037 & N.S. & N.S. & N.S. & N.S. & 11.34 & 0.001 & N.S. & N.S. & N.S. & N.S. & 2 \\
\hline Araliaceae & Schefflera distractiflora (Harms) Frodin & 2.22 & 0.94 & N.S. & N.S. & N.S. & N.S. & N.S. & N.S. & N.S. & N.S. & N.S. & N.S. & N.S. & N.S. & 2 \\
\hline Araliaceae & Schefflera macrocarpa (Cham. \& Schltdl.) Frodin & -2.02 & 0.44 & 5.52 & 0.021 & 26.95 & $<0.001$ & N.S. & N.S. & 11.68 & 0.001 & N.S. & N.S. & N.S. & N.S. & 12 \\
\hline Araliaceae & $\begin{array}{l}\text { Schefflera morototoni (Aubl.) Maguire et al. } \\
\text { Schefflera vinosa (Cham. \& Schltdl.) Frodin \& }\end{array}$ & -3.04 & -2.25 & N.S. & N.S. & N.S. & N.S. & N.S. & N.S. & N.S. & N.S. & N.S. & N.S. & N.S. & N.S. & 4 \\
\hline Araliaceae & Fiaschi & -1.64 & -0.29 & N.S. & N.S. & N.S. & N.S. & N.S. & N.S. & 5.49 & 0.022 & N.S. & N.S. & N.S. & N.S. & 7 \\
\hline Asteraceae & Eremanthus glomeratus Less. & -2.47 & 1.09 & 7.53 & 0.007 & 5.88 & 0.017 & 4.11 & 0.045 & N.S. & N.S. & N.S. & N.S. & N.S. & N.S. & 4 \\
\hline Asteraceae & Gochnatia pulchra Cabrera & -4.77 & 0.15 & N.S. & N.S. & N.S. & N.S. & 5.55 & 0.020 & N.S. & N.S. & N.S. & N.S. & N.S. & N.S. & 2 \\
\hline Asteraceae & Piptocarpha rotundifolia (Less.) Baker & -0.63 & 0.23 & N.S. & N.S. & 4.49 & 0.037 & N.S. & N.S. & N.S. & N.S. & N.S. & N.S. & N.S. & N.S. & 24 \\
\hline Asteraceae & Vernonanthura ferruginea (Less.) H.Rob. & 1.36 & 0.12 & N.S. & N.S. & N.S. & N.S. & N.S. & N.S. & N.S. & N.S. & 4.52 & 0.036 & N.S. & N.S. & 15 \\
\hline Bignoniaceae & Cybistax antisyphilitica (Mart.) Mart. & 0.00 & 0.83 & N.S. & N.S. & N.S. & N.S. & 5.78 & 0.018 & N.S. & N.S. & 4.38 & 0.039 & N.S. & N.S. & 24 \\
\hline Bignoniaceae & Handroanthus impetiginosus Mattos & 0.85 & -1.20 & 6.73 & 0.011 & N.S. & N.S. & N.S. & N.S. & N.S. & N.S. & N.S. & N.S. & 6.08 & 0.016 & 10 \\
\hline Bignoniaceae & Handroanthus ochraceus (Cham.) Mattos & 0.32 & 0.13 & N.S. & N.S. & N.S. & N.S. & N.S. & N.S. & N.S. & N.S. & N.S. & N.S. & N.S. & N.S. & 55 \\
\hline Bignoniaceae & Handroanthus serratifolius (A.H.Gentry) S.Grose & 1.07 & 0.51 & N.S. & N.S. & N.S. & N.S. & N.S. & N.S. & N.S. & N.S. & 13.39 & $<0.001$ & N.S. & N.S. & 19 \\
\hline Bignoniaceae & Jacaranda brasiliana (Lam.) Pers. & 2.76 & -0.68 & 7.16 & 0.009 & 5.99 & 0.016 & 9.65 & 0.003 & N.S. & N.S. & N.S. & N.S. & N.S. & N.S. & 14 \\
\hline Bignoniaceae & Jacaranda caroba (Vell.) DC. & -4.77 & 0.15 & N.S. & N.S. & N.S. & N.S. & 5.55 & 0.020 & N.S. & N.S. & N.S. & N.S. & N.S. & N.S. & 2 \\
\hline Bignoniaceae & $\begin{array}{l}\text { Jacaranda cuspidifolia Mart. } \\
\text { Tabebuia aurea (Silva Manso) Benth. \& Hook.f. ex }\end{array}$ & 1.17 & -2.41 & 9.48 & 0.002 & N.S. & N.S. & N.S. & N.S. & 5.19 & 0.025 & 6.59 & 0.012 & 7.17 & 0.009 & 4 \\
\hline Bignoniaceae & S.Moore & 0.65 & -0.14 & N.S. & N.S. & N.S. & N.S. & 17.38 & $<0.001$ & 8.38 & 0.005 & N.S. & N.S. & N.S. & N.S. & 57 \\
\hline Bignoniaceae & Tabebuia roseoalba (Ridl.) Sandwith & 1.25 & -2.53 & 6.93 & 0.010 & N.S. & N.S. & 5.16 & 0.026 & N.S. & N.S. & N.S. & N.S. & 9.45 & 0.003 & 7 \\
\hline Bignoniaceae & Zeyheria montana Mart. & 1.10 & -0.51 & N.S. & N.S. & N.S. & N.S. & N.S. & N.S. & 4.23 & 0.042 & N.S. & N.S. & 14.34 & $<0.001$ & 23 \\
\hline Bixaceae & Cochlospermum vitifolium (Willd.) Spreng. & 2.69 & -1.99 & 11.04 & 0.001 & N.S. & N.S. & N.S. & N.S. & N.S. & N.S. & N.S. & N.S. & N.S. & N.S. & 6 \\
\hline Boraginaceae & Cordia glabrata (Mart.) A.DC. & 2.27 & -0.06 & 11.40 & 0.001 & N.S. & N.S. & 4.06 & 0.047 & 10.29 & 0.002 & N.S. & N.S. & N.S. & N.S. & 20 \\
\hline Boraginaceae & Cordia sellowiana Cham. & 1.17 & -0.26 & N.S. & N.S. & N.S. & N.S. & N.S. & N.S. & N.S. & N.S. & N.S. & N.S. & N.S. & N.S. & 3 \\
\hline Boraginaceae & Cordia superba Cham. & 0.85 & 1.12 & N.S. & N.S. & N.S. & N.S. & N.S. & N.S. & N.S. & N.S. & N.S. & N.S. & N.S. & N.S. & 3 \\
\hline Burseraceae & Protium heptaphyllum (Aubl.) Marchand & 1.36 & -0.50 & 14.42 & $<0.001$ & N.S. & N.S. & N.S. & N.S. & N.S. & N.S. & 12.55 & 0.001 & N.S. & N.S. & 31 \\
\hline Burseraceae & Protium ovatum Engl. & 0.15 & 0.74 & 4.41 & 0.038 & 11.09 & 0.001 & N.S. & N.S. & 6.06 & 0.016 & 5.39 & 0.023 & N.S. & N.S. & 6 \\
\hline
\end{tabular}




\begin{tabular}{|c|c|c|c|c|c|c|c|c|c|c|c|c|c|c|c|c|}
\hline Família & Espécie & PC1 & PC2 & $\begin{array}{c}\text { SB } \\
F_{1,78} \\
\end{array}$ & $p$ & $\begin{array}{c}\mathbf{m} \\
\mathrm{F}_{1,78} \\
\end{array}$ & $p$ & $\begin{array}{c}\mathrm{pH} \\
\mathrm{F}_{1,78} \\
\end{array}$ & $p$ & $\begin{array}{c}\text { Bio05 } \\
F_{1,78}\end{array}$ & $p$ & $\begin{array}{c}\text { Bio23 } \\
F_{1,78}\end{array}$ & $p$ & $\begin{array}{c}\text { Bio30 } \\
F_{1,78}\end{array}$ & $p$ & $\mathbf{N}$ \\
\hline Burseraceae & Tetragastris altissima (Aubl.) Swart & 0.61 & 0.78 & N.S. & N.S. & N.S. & N.S. & 5.72 & 0.019 & N.S. & N.S. & N.S. & N.S. & N.S. & N.S. & 2 \\
\hline Calophyllaceae & Kielmeyera coriacea Mart. \& Zucc. & 0.22 & 0.11 & 7.29 & 0.008 & N.S. & N.S. & 10.28 & 0.002 & N.S. & N.S. & N.S. & N.S. & N.S. & N.S. & 61 \\
\hline Calophyllaceae & Kielmeyera lathrophyton Saddi & 0.62 & 1.46 & 13.19 & 0.001 & N.S. & N.S. & N.S. & N.S. & 8.95 & 0.004 & N.S. & N.S. & 5.52 & 0.012 & 15 \\
\hline Calophyllaceae & Kielmeyera rubriflora Cambess. & 0.29 & 0.25 & N.S. & N.S. & N.S. & N.S. & N.S. & N.S. & N.S. & N.S. & N.S. & N.S. & N.S. & N.S. & 17 \\
\hline Calophyllaceae & Kielmeyera speciosa A.St.-Hil. & 0.34 & 1.02 & N.S. & N.S. & 5.40 & 0.023 & N.S. & N.S. & 6.26 & 0.014 & N.S. & N.S. & 5.15 & 0.025 & 6 \\
\hline Caryocaraceae & Caryocar brasiliense Cambess. & -0.04 & 0.02 & N.S. & N.S. & N.S. & N.S. & N.S. & N.S. & 7.28 & 0.009 & N.S. & N.S. & N.S. & N.S. & 50 \\
\hline Caryocaraceae & Caryocar coriaceum Wittm. & -0.49 & 1.87 & N.S. & N.S. & N.S. & N.S. & N.S. & N.S. & N.S. & N.S. & N.S. & N.S. & N.S. & N.S. & 4 \\
\hline Caryocaraceae & Caryocar cuneatum Wittm. & 2.15 & 0.73 & N.S. & N.S. & 12.39 & 0.001 & N.S. & N.S. & N.S. & N.S. & 5.33 & 0.024 & N.S. & N.S. & 12 \\
\hline Celastraceae & Plenckia populnea Reissek & -0.76 & 0.91 & 4.63 & 0.034 & 6.97 & 0.010 & N.S. & N.S. & N.S. & N.S. & N.S. & N.S. & N.S. & N.S. & 14 \\
\hline Celastraceae & Salacia crassifolia (Mart. ex Schult.) G.Don & 1.22 & 0.66 & N.S. & N.S. & 25.55 & $<0.001$ & N.S. & N.S. & N.S. & N.S. & N.S. & N.S. & 5.73 & 0.019 & 34 \\
\hline Celastraceae & Salacia elliptica (Mart. ex Schult.) G.Don & 0.52 & 0.52 & N.S. & N.S. & N.S. & N.S. & N.S. & N.S. & N.S. & N.S. & N.S. & N.S. & 4.07 & 0.047 & 8 \\
\hline Chrysobalanaceae & Couepia grandiflora (Mart. \& Zucc.) Benth. & 0.29 & 0.29 & N.S. & N.S. & N.S. & N.S. & N.S. & N.S. & N.S. & N.S. & N.S. & N.S. & N.S. & N.S. & 37 \\
\hline Chrysobalanaceae & Hirtella ciliata Mart. \& Zucc. & 0.98 & 1.34 & 5.65 & 0.020 & 9.57 & 0.003 & 5.34 & 0.023 & 9.81 & 0.002 & 21.10 & $<0.001$ & N.S. & N.S. & 19 \\
\hline Chrysobalanaceae & Hirtella glandulosa Spreng. & 0.63 & 0.45 & N.S. & N.S. & N.S. & N.S. & N.S. & N.S. & N.S. & N.S. & N.S. & N.S. & N.S. & N.S. & 17 \\
\hline Chrysobalanaceae & Hirtella gracilipes (Hook.f.) Prance & -0.98 & 0.36 & N.S. & N.S. & 4.51 & 0.036 & N.S. & N.S. & N.S. & N.S. & N.S. & N.S. & N.S. & N.S. & 7 \\
\hline Chrysobalanaceae & Licania gardneri (Hook.f.) Fritsch & 2.58 & -0.63 & N.S. & N.S. & N.S. & N.S. & N.S. & N.S. & N.S. & N.S. & N.S. & N.S. & N.S. & N.S. & 2 \\
\hline Chrysobalanaceae & Licania humilis Cham. \& Schltdl. & -3.08 & 0.18 & 7.39 & 0.008 & 25.48 & $<0.001$ & N.S. & N.S. & 6.17 & 0.015 & N.S. & N.S. & 13.31 & $<0.001$ & 11 \\
\hline Chrysobalanaceae & Licania sclerophylla (Hook.f.) Fritsch & 4.13 & -3.17 & 9.86 & 0.002 & N.S. & N.S. & N.S. & N.S. & N.S. & N.S. & N.S. & N.S. & N.S. & N.S. & 3 \\
\hline Clusiaceae & Clusia sellowiana Schltdl. & -2.88 & -0.72 & N.S. & N.S. & N.S. & N.S. & N.S. & N.S. & 8.33 & 0.005 & N.S. & N.S. & N.S. & N.S. & 2 \\
\hline Combretaceae & Buchenavia tetraphylla (Aubl.) R.A.Howard & -1.43 & 0.77 & N.S. & N.S. & N.S. & N.S. & N.S. & N.S. & N.S. & N.S. & N.S. & N.S. & 7.19 & 0.009 & 2 \\
\hline Combretaceae & Buchenavia tomentosa Eichler & 0.90 & -0.82 & 10.53 & 0.002 & N.S. & N.S. & 5.14 & 0.026 & 7.94 & 0.006 & N.S. & N.S. & N.S. & N.S. & 21 \\
\hline Combretaceae & Combretum mellifluum Eichler & 0.71 & 0.64 & 5.59 & 0.020 & 19.24 & $<0.001$ & N.S. & N.S. & N.S. & N.S. & 5.46 & 0.022 & N.S. & N.S. & 13 \\
\hline Combretaceae & Terminalia argentea Mart. & 0.48 & -0.67 & 7.74 & 0.007 & N.S. & N.S. & 8.04 & 0.006 & N.S. & N.S. & N.S. & N.S. & N.S. & N.S. & 32 \\
\hline Combretaceae & Terminalia fagifolia Mart. & 1.02 & 0.96 & 9.85 & 0.002 & 15.90 & $<0.001$ & N.S. & N.S. & N.S. & N.S. & 7.87 & 0.006 & 14.13 & $<0.001$ & 24 \\
\hline Combretaceae & Terminalia glabrescens Mart. & -2.31 & -0.11 & N.S. & N.S. & 14.27 & $<0.001$ & N.S. & N.S. & 7.98 & 0.006 & N.S. & N.S. & 5.71 & 0.019 & 10 \\
\hline Combretaceae & Terminalia phaeocarpa Eichler & 0.77 & -0.30 & N.S. & N.S. & N.S. & N.S. & N.S. & N.S. & N.S. & N.S. & N.S. & N.S. & N.S. & N.S. & 4 \\
\hline Connaraceae & Connarus suberosus Planch. & 0.28 & 0.52 & 23.84 & $<0.001$ & N.S. & N.S. & N.S. & N.S. & N.S. & N.S. & 4.56 & 0.036 & 6.14 & 0.015 & 69 \\
\hline Connaraceae & Rourea induta Planch. & 0.64 & 0.41 & N.S. & N.S. & N.S. & N.S. & N.S. & N.S. & 8.89 & 0.004 & 8.82 & 0.004 & N.S. & N.S. & 38 \\
\hline Dichapetalaceae & Tapura amazonica Poepp. \& Endl. & 2.45 & 1.14 & N.S. & N.S. & N.S. & N.S. & N.S. & N.S. & N.S. & N.S. & N.S. & N.S. & N.S. & N.S. & 3 \\
\hline
\end{tabular}




\begin{tabular}{|c|c|c|c|c|c|c|c|c|c|c|c|c|c|c|c|c|}
\hline Família & Espécie & PC1 & PC2 & $\begin{array}{c}\text { SB } \\
F_{1,78} \\
\end{array}$ & $p$ & $\begin{array}{c}\mathbf{m} \\
\mathrm{F}_{1,78} \\
\end{array}$ & $p$ & $\begin{array}{c}\text { pH } \\
\mathrm{F}_{1,78} \\
\end{array}$ & $p$ & $\begin{array}{c}\text { Bio05 } \\
F_{1,78}\end{array}$ & $p$ & $\begin{array}{c}\text { Bio23 } \\
F_{1,78}\end{array}$ & $p$ & $\begin{array}{c}\text { Bio30 } \\
F_{1,78} \\
\end{array}$ & $p$ & $\mathbf{N}$ \\
\hline Dilleniaceae & Curatella americana L. & 0.90 & -0.20 & 18.01 & $<0.001$ & N.S. & N.S. & N.S. & N.S. & 9.56 & 0.003 & N.S. & N.S. & N.S. & N.S. & 50 \\
\hline Dilleniaceae & Davilla elliptica A.St.-Hil. & 0.88 & 0.46 & N.S. & N.S. & 9.03 & 0.004 & N.S. & N.S. & 4.01 & 0.005 & N.S. & N.S. & N.S. & N.S. & 55 \\
\hline Ebenaceae & Diospyros coccolobifolia Mart. ex Miq. & 0.87 & 1.05 & N.S. & N.S. & N.S. & N.S. & 10.56 & 0.002 & N.S. & N.S. & 15.49 & $<0.001$ & N.S. & N.S. & 4 \\
\hline Ebenaceae & Diospyros hispida A.DC. & 0.83 & 0.47 & N.S. & N.S. & N.S. & N.S. & 4.89 & 0.029 & 9.62 & 0.003 & N.S. & N.S. & N.S. & N.S. & 54 \\
\hline Ebenaceae & Diospyros sericea A.DC. & 1.29 & -0.06 & N.S. & N.S. & N.S. & N.S. & N.S. & N.S. & N.S. & N.S. & N.S. & N.S. & N.S. & N.S. & 11 \\
\hline Erythroxylaceae & Erythroxylum betulaceum Mart. & 1.24 & 1.39 & 7.27 & 0.008 & 15.85 & $<0.001$ & N.S. & N.S. & 3.99 & 0.049 & 7.73 & 0.006 & N.S. & N.S. & 6 \\
\hline Erythroxylaceae & Erythroxylum daphnites Mart. & 0.77 & -0.15 & 6.20 & 0.014 & N.S. & N.S. & N.S. & N.S. & N.S. & N.S. & N.S. & N.S. & N.S. & N.S. & 13 \\
\hline Erythroxylaceae & Erythroxylum deciduum A.St.-Hil. & 0.14 & 0.69 & 13.34 & $<0.001$ & N.S. & N.S. & 5.70 & 0.019 & N.S. & N.S. & N.S. & N.S. & N.S. & N.S. & 30 \\
\hline Erythroxylaceae & Erythroxylum engleri O.E.Schulz & -0.47 & 0.80 & N.S. & N.S. & N.S. & N.S. & N.S. & N.S. & N.S. & N.S. & N.S. & N.S. & N.S. & N.S. & 4 \\
\hline Erythroxylaceae & Erythroxylum pelleterianum A.St.-Hil. & -4.28 & -0.35 & N.S. & N.S. & 5.13 & 0.026 & 11.44 & 0.001 & 8.29 & 0.005 & N.S. & N.S. & N.S. & N.S. & 3 \\
\hline Erythroxylaceae & Erythroxylum suberosum A.St.-Hil. & 0.16 & 0.05 & N.S. & N.S. & N.S. & N.S. & N.S. & N.S. & N.S. & N.S. & 6.16 & 0.015 & N.S. & N.S. & 55 \\
\hline Erythroxylaceae & Erythroxylum tortuosum Mart. & -0.24 & 0.33 & N.S. & N.S. & 5.35 & 0.023 & N.S. & N.S. & N.S. & N.S. & N.S. & N.S. & N.S. & N.S. & 21 \\
\hline Erythroxylaceae & Erythroxylum vacciniifolium Mart. & 2.14 & -1.18 & N.S. & N.S. & N.S. & N.S. & N.S. & N.S. & N.S. & N.S. & N.S. & N.S. & N.S. & N.S. & 3 \\
\hline Euphorbiaceae & Alchornea discolor Poepp. & 1.49 & 0.84 & N.S. & N.S. & N.S. & N.S. & N.S. & N.S. & 6.49 & 0.013 & 7.90 & 0.006 & 4.72 & 0.033 & 6 \\
\hline Euphorbiaceae & Croton floribundus Spreng. & -1.63 & -0.86 & N.S. & N.S. & N.S. & N.S. & N.S. & N.S. & N.S. & N.S. & N.S. & N.S. & N.S. & N.S. & 3 \\
\hline Euphorbiaceae & Mabea fistulifera Mart. & 0.00 & 0.12 & N.S. & N.S. & N.S. & N.S. & 4.10 & 0.046 & N.S. & N.S. & 5.70 & 0.019 & N.S. & N.S. & 3 \\
\hline Euphorbiaceae & Mabea pohliana (Benth.) Müll.Arg. & 0.61 & 0.78 & N.S. & N.S. & N.S. & N.S. & 5.72 & 0.019 & N.S. & N.S. & N.S. & N.S. & N.S. & N.S. & 2 \\
\hline Euphorbiaceae & Maprounea guianensis Aubl. & -0.57 & 0.64 & 4.54 & 0.036 & N.S. & N.S. & 5.77 & 0.018 & N.S. & N.S. & N.S. & N.S. & N.S. & N.S. & 23 \\
\hline Fabaceae & Albizia niopoides (Spruce ex Benth.) Burkart & 1.24 & 1.55 & N.S. & N.S. & N.S. & N.S. & N.S. & N.S. & N.S. & N.S. & N.S. & N.S. & N.S. & N.S. & 2 \\
\hline Fabaceae & Anadenanthera colubrina (Vell.) Brenan & 1.55 & -1.08 & 5.56 & 0.021 & N.S. & N.S. & N.S. & N.S. & N.S. & N.S. & N.S. & N.S. & N.S. & N.S. & 8 \\
\hline Fabaceae & Anadenanthera peregrina (L.) Speg. & -2.44 & -1.05 & N.S. & N.S. & N.S. & N.S. & N.S. & N.S. & N.S. & N.S. & N.S. & N.S. & 6.26 & 0.014 & 7 \\
\hline Fabaceae & Andira cordata Arroyo ex R.T.Penn. \& H.C.Lima & 1.70 & 1.65 & N.S. & N.S. & N.S. & N.S. & N.S. & N.S. & 10.97 & 0.001 & N.S. & N.S. & N.S. & N.S. & 10 \\
\hline Fabaceae & Andira cujabensis Benth. & 1.01 & -0.24 & 5.45 & 0.022 & N.S. & N.S. & N.S. & N.S. & 7.13 & 0.009 & N.S. & N.S. & N.S. & N.S. & 26 \\
\hline Fabaceae & Andira vermifuga (Mart.) Benth. & 0.66 & 0.86 & 15.63 & $<0.001$ & 6.32 & 0.014 & N.S. & N.S. & N.S. & N.S. & N.S. & N.S. & N.S. & N.S. & 42 \\
\hline Fabaceae & Apuleia leiocarpa (Vogel) J.F.Macbr. & -0.21 & 1.38 & N.S. & N.S. & N.S. & N.S. & N.S. & N.S. & N.S. & N.S. & N.S. & N.S. & N.S. & N.S. & 2 \\
\hline Fabaceae & Bauhinia brevipes Vogel & 1.12 & -3.13 & N.S. & N.S. & N.S. & N.S. & N.S. & N.S. & N.S. & N.S. & N.S. & N.S. & N.S. & N.S. & 3 \\
\hline Fabaceae & Bauhinia pulchella Benth. & 1.70 & -0.69 & N.S. & N.S. & N.S. & N.S. & N.S. & N.S. & N.S. & N.S. & N.S. & N.S. & 5.07 & 0.027 & 8 \\
\hline Fabaceae & Bauhinia rufa (Bong.) Steud. & 0.79 & -1.38 & 10.56 & 0.002 & N.S. & N.S. & N.S. & N.S. & N.S. & N.S. & N.S. & N.S. & N.S. & N.S. & 11 \\
\hline Fabaceae & Bowdichia virgilioides Kunth & 0.26 & 0.35 & 5.57 & $<0.001$ & N.S. & N.S. & N.S. & N.S. & N.S. & N.S. & N.S. & N.S. & N.S. & N.S. & 72 \\
\hline
\end{tabular}




\begin{tabular}{|c|c|c|c|c|c|c|c|c|c|c|c|c|c|c|c|c|}
\hline Família & Espécie & PC1 & PC2 & $\begin{array}{c}\text { SB } \\
F_{1,78}\end{array}$ & $p$ & $\begin{array}{c}m \\
F_{1,78}\end{array}$ & $p$ & $\begin{array}{c}\mathrm{pH} \\
\mathrm{F}_{1,78}\end{array}$ & $p$ & $\begin{array}{c}\text { Bio05 } \\
F_{1,78} \\
\end{array}$ & $p$ & $\begin{array}{c}\text { Bio23 } \\
F_{1,78} \\
\end{array}$ & $p$ & $\begin{array}{c}\text { Bio30 } \\
F_{1,78} \\
\end{array}$ & $p$ & $\mathbf{N}$ \\
\hline Fabaceae & Cenostigma macrophyllum Tul. & 2.13 & 1.19 & N.S. & N.S. & N.S. & N.S. & N.S. & N.S. & 15.30 & $<0.001$ & 9.81 & 0.002 & N.S. & N.S. & 12 \\
\hline Fabaceae & $\begin{array}{l}\text { Centrolobium tomentosum Guillem. ex Benth. } \\
\text { Chamaecrista orbiculata (Benth.) H.S.Irwin \& }\end{array}$ & -3.46 & -2.28 & N.S. & N.S. & N.S. & N.S. & N.S. & N.S. & 9.62 & 0.002 & N.S. & N.S. & N.S. & N.S. & 2 \\
\hline Fabaceae & Barneby & -1.64 & 0.18 & N.S. & N.S. & N.S. & N.S. & N.S. & N.S. & N.S. & N.S. & N.S. & N.S. & N.S. & N.S. & 3 \\
\hline Fabaceae & Copaifera langsdorffii Desf. & -0.29 & -0.14 & N.S. & N.S. & N.S. & N.S. & N.S. & N.S. & 12.42 & 0.001 & N.S. & N.S. & N.S. & N.S. & 42 \\
\hline Fabaceae & Copaifera martii Hayne & 1.95 & 0.28 & N.S. & N.S. & 12.89 & 0.001 & N.S. & N.S. & N.S. & N.S. & N.S. & N.S. & 6.36 & 0.013 & 20 \\
\hline Fabaceae & Dalbergia miscolobium Benth. & -0.36 & 0.19 & N.S. & N.S. & N.S. & N.S. & N.S. & N.S. & N.S. & N.S. & N.S. & N.S. & N.S. & N.S. & 32 \\
\hline Fabaceae & Dimorphandra gardneriana Tul. & 0.15 & 1.30 & N.S. & N.S. & N.S. & N.S. & N.S. & N.S. & N.S. & N.S. & 6.34 & 0.013 & N.S. & N.S. & 6 \\
\hline Fabaceae & Dimorphandra mollis Benth. & 0.41 & 0.43 & 7.65 & 0.007 & N.S. & N.S. & N.S. & N.S. & N.S. & N.S. & N.S. & N.S. & 10.15 & 0.002 & 66 \\
\hline Fabaceae & Dipteryx alata Vogel & 0.75 & -0.86 & 24.99 & $<0.001$ & N.S. & N.S. & N.S. & N.S. & 5.65 & 0.019 & N.S. & N.S. & N.S. & N.S. & 26 \\
\hline Fabaceae & Diptychandra aurantiaca Tul. & -2.84 & -1.29 & N.S. & N.S. & N.S. & N.S. & N.S. & N.S. & N.S. & N.S. & N.S. & N.S. & 9.72 & 0.003 & 5 \\
\hline Fabaceae & Enterolobium contortisiliquum (Vell.) Morong & -0.30 & 0.05 & N.S. & N.S. & N.S. & N.S. & N.S. & N.S. & N.S. & N.S. & 13.97 & $<0.001$ & N.S. & N.S. & 3 \\
\hline Fabaceae & Enterolobium gummiferum (Mart.) J.F.Macbr. & 0.14 & 0.69 & 8.34 & 0.005 & N.S. & N.S. & N.S. & N.S. & 4.05 & 0.047 & N.S. & N.S. & N.S. & N.S. & 36 \\
\hline Fabaceae & Hymenaea courbaril L. & 1.47 & -0.48 & 4.90 & 0.029 & N.S. & N.S. & N.S. & N.S. & N.S. & N.S. & N.S. & N.S. & N.S. & N.S. & 9 \\
\hline Fabaceae & Hymenaea eriogyne Benth. & 1.73 & 1.23 & N.S. & N.S. & N.S. & N.S. & N.S. & N.S. & 13.87 & $<0.001$ & N.S. & N.S. & N.S. & N.S. & 4 \\
\hline Fabaceae & Hymenaea maranhensis Y.T.Lee \& Langenh. & 1.08 & 1.16 & N.S. & N.S. & N.S. & N.S. & 5.45 & 0.022 & N.S. & N.S. & 11.90 & 0.001 & N.S. & N.S. & 2 \\
\hline Fabaceae & Hymenaea martiana Hayne & -2.24 & 0.93 & N.S. & N.S. & N.S. & N.S. & N.S. & N.S. & N.S. & N.S. & 6.81 & 0.011 & N.S. & N.S. & 3 \\
\hline Fabaceae & Hymenaea stigonocarpa Mart. ex Hayne & 0.45 & 0.02 & N.S. & N.S. & N.S. & N.S. & N.S. & N.S. & N.S. & N.S. & 10.52 & 0.002 & N.S. & N.S. & 70 \\
\hline Fabaceae & Leptolobium dasycarpum Vogel & 0.36 & 0.20 & N.S. & N.S. & N.S. & N.S. & 6.81 & 0.010 & N.S. & N.S. & N.S. & N.S. & 10.75 & 0.002 & 55 \\
\hline Fabaceae & Leptolobium elegans Vogel & -1.53 & 0.28 & 6.21 & 0.015 & 8.87 & 0.004 & 4.41 & 0.038 & 4.43 & 0.038 & 4.55 & 0.036 & N.S. & N.S. & 9 \\
\hline Fabaceae & Luetzelburgia praecox (Harms) Harms & -0.06 & -0.26 & N.S. & N.S. & N.S. & N.S. & N.S. & N.S. & N.S. & N.S. & N.S. & N.S. & N.S. & N.S. & 3 \\
\hline Fabaceae & Machaerium acutifolium Vogel & 0.55 & 0.33 & N.S. & N.S. & N.S. & N.S. & N.S. & N.S. & N.S. & N.S. & 5.12 & 0.026 & N.S. & N.S. & 49 \\
\hline Fabaceae & Machaerium hirtum (Vell.) Stellfeld & 1.79 & 0.62 & N.S. & N.S. & N.S. & N.S. & N.S. & N.S. & N.S. & N.S. & N.S. & N.S. & N.S. & N.S. & 5 \\
\hline Fabaceae & $\begin{array}{l}\text { Machaerium opacum Vogel } \\
\text { Martiodendron mediterraneum (Mart. ex Benth.) }\end{array}$ & 0.19 & 0.34 & 8.56 & 0.004 & N.S. & N.S. & 9.71 & 0.003 & N.S. & N.S. & N.S. & N.S. & 13.72 & $<0.001$ & 41 \\
\hline Fabaceae & R.C.Koeppen & 0.61 & 0.78 & N.S. & N.S. & N.S. & N.S. & 5.73 & 0.019 & N.S. & N.S. & N.S. & N.S. & N.S. & N.S. & 2 \\
\hline Fabaceae & Mimosa claussenii Benth. & 0.61 & -0.25 & N.S. & N.S. & N.S. & N.S. & N.S. & N.S. & N.S. & N.S. & N.S. & N.S. & N.S. & N.S. & 4 \\
\hline Fabaceae & Mimosa sericantha Benth. & -1.29 & 0.45 & N.S. & N.S. & N.S. & N.S. & N.S. & N.S. & N.S. & N.S. & N.S. & N.S. & N.S. & N.S. & 2 \\
\hline Fabaceae & Parkia platycephala Benth. & 1.75 & 0.93 & N.S. & N.S. & 11.21 & 0.001 & 4.28 & 0.042 & 6.18 & 0.015 & 17.42 & $<0.001$ & N.S. & N.S. & 13 \\
\hline Fabaceae & Peltogyne confertiflora (Mart. ex Hayne) Benth. & 0.19 & 0.21 & N.S. & N.S. & N.S. & N.S. & N.S. & N.S. & N.S. & N.S. & N.S. & N.S. & N.S. & N.S. & 8 \\
\hline
\end{tabular}




\begin{tabular}{|c|c|c|c|c|c|c|c|c|c|c|c|c|c|c|c|c|}
\hline Família & Espécie & PC1 & PC2 & $\begin{array}{c}S B \\
F_{1,78}\end{array}$ & $p$ & $\begin{array}{c}m \\
F_{1,78}\end{array}$ & $p$ & $\begin{array}{c}\mathrm{pH} \\
\mathrm{F}_{1,78}\end{array}$ & $p$ & $\begin{array}{c}\text { Bio05 } \\
F_{1,78} \\
\end{array}$ & $p$ & $\begin{array}{c}\text { Bio23 } \\
F_{1,78} \\
\end{array}$ & $p$ & $\begin{array}{c}\text { Bio30 } \\
F_{1,78}\end{array}$ & $p$ & $\mathbf{N}$ \\
\hline Fabaceae & Plathymenia reticulata Benth. & -0.23 & -0.56 & N.S. & N.S. & N.S. & N.S. & N.S. & N.S. & N.S. & N.S. & N.S. & N.S. & 7.63 & 0.007 & 46 \\
\hline Fabaceae & Platypodium elegans Vogel & -0.34 & -0.43 & N.S. & N.S. & 10.47 & 0.002 & N.S. & N.S. & 8.14 & 0.006 & N.S. & N.S. & 6.83 & 0.011 & 15 \\
\hline Fabaceae & Pterodon emarginatus Vogel & 1.18 & 0.47 & N.S. & N.S. & 30.94 & $<0.001$ & N.S. & N.S. & N.S. & N.S. & N.S. & N.S. & 9.27 & 0.003 & 28 \\
\hline Fabaceae & Pterodon pubescens (Benth.) Benth. & -0.25 & -0.26 & N.S. & N.S. & N.S. & N.S. & N.S. & N.S. & N.S. & N.S. & N.S. & N.S. & N.S. & N.S. & 18 \\
\hline Fabaceae & Pterogyne nitens Tul. & 1.39 & -2.03 & 4.89 & 0.029 & N.S. & N.S. & N.S. & N.S. & N.S. & N.S. & 6.94 & 0.010 & 11.15 & 0.001 & 2 \\
\hline Fabaceae & Senegalia polyphylla (DC.) Britton \& Rose & -1.92 & 1.00 & N.S. & N.S. & N.S. & N.S. & 6.99 & 0.010 & N.S. & N.S. & N.S. & N.S. & N.S. & N.S. & 3 \\
\hline Fabaceae & Senna rugosa (G.Don) H.S.Irwin \& Barneby & -4.77 & 0.15 & N.S. & N.S. & N.S. & N.S. & 5.55 & 0.021 & N.S. & N.S. & N.S. & N.S. & N.S. & N.S. & 2 \\
\hline Fabaceae & Senna silvestris (Vell.) H.S.Irwin \& Barneby & 2.18 & -2.63 & 10.38 & 0.002 & N.S. & N.S. & N.S. & N.S. & N.S. & N.S. & N.S. & N.S. & N.S. & N.S. & 4 \\
\hline Fabaceae & Senna velutina (Vogel) H.S.Irwin \& Barneby & -0.21 & 0.71 & N.S. & N.S. & N.S. & N.S. & N.S. & N.S. & N.S. & N.S. & N.S. & N.S. & N.S. & N.S. & 2 \\
\hline Fabaceae & Stryphnodendron adstringens (Mart.) Coville & -0.98 & 1.64 & 13.26 & $<0.001$ & 25.00 & $<0.001$ & N.S. & N.S. & 9.02 & 0.004 & N.S. & N.S. & N.S. & N.S. & 14 \\
\hline Fabaceae & Stryphnodendron coriaceum Benth. & 0.51 & 0.03 & N.S. & N.S. & N.S. & N.S. & N.S. & N.S. & 4.92 & 0.029 & 13.54 & $<0.001$ & N.S. & N.S. & 9 \\
\hline Fabaceae & Stryphnodendron polyphyllum Mart. & -1.05 & 0.87 & 8.46 & 0.004 & 10.12 & 0.002 & 8.64 & 0.004 & 6.03 & 0.016 & N.S. & N.S. & 5.30 & 0.024 & 7 \\
\hline Fabaceae & Stryphnodendron rotundifolium Mart. & 0.74 & 0.67 & N.S. & N.S. & 8.21 & 0.005 & 5.48 & 0.022 & 7.64 & 0.007 & N.S. & N.S. & N.S. & N.S. & 41 \\
\hline Fabaceae & Swartzia apetala Raddi & -0.44 & -0.43 & N.S. & N.S. & N.S. & N.S. & N.S. & N.S. & N.S. & N.S. & N.S. & N.S. & N.S. & N.S. & 2 \\
\hline Fabaceae & Tachigali aurea Tul. & 0.64 & 0.03 & N.S. & N.S. & N.S. & N.S. & 8.71 & 0.004 & N.S. & N.S. & N.S. & N.S. & N.S. & N.S. & 53 \\
\hline Fabaceae & Tachigali subvelutina (Benth.) Oliveira-Filho & 0.39 & 0.98 & 24.98 & $<0.001$ & 4.56 & 0.036 & N.S. & N.S. & 5.41 & 0.022 & 8.58 & 0.004 & N.S. & N.S. & 44 \\
\hline Fabaceae & Tachigali vulgaris L.G.Silva \& H.C.Lima & 0.02 & 1.46 & N.S. & N.S. & N.S. & N.S. & 23.47 & $<0.001$ & N.S. & N.S. & 11.97 & 0.001 & N.S. & N.S. & 6 \\
\hline Fabaceae & Vatairea macrocarpa (Benth.) Ducke & 0.75 & 0.49 & N.S. & N.S. & 14.14 & $<0.001$ & N.S. & N.S. & 14.00 & $<0.001$ & N.S. & N.S. & N.S. & N.S. & 65 \\
\hline Icacinaceae & Emmotum nitens (Benth.) Miers & 0.98 & 0.55 & N.S. & N.S. & 7.92 & 0.006 & N.S. & N.S. & 5.36 & 0.023 & N.S. & N.S. & N.S. & N.S. & 39 \\
\hline Lacistemataceae & Lacistema hasslerianum Chodat & -2.10 & 1.04 & N.S. & N.S. & 4.22 & 0.043 & N.S. & N.S. & N.S. & N.S. & N.S. & N.S. & N.S. & N.S. & 2 \\
\hline Lamiaceae & Aegiphila verticillata Vell. & -1.34 & 0.27 & N.S. & N.S. & 6.95 & 0.010 & N.S. & N.S. & N.S. & N.S. & N.S. & N.S. & N.S. & N.S. & 13 \\
\hline Lamiaceae & Hyptidendron canum (Pohl ex Benth.) Harley & -2.81 & 1.79 & N.S. & N.S. & 7.78 & 0.007 & N.S. & N.S. & N.S. & N.S. & N.S. & N.S. & N.S. & N.S. & 2 \\
\hline Lamiaceae & Vitex cymosa Bertero ex Spreng. & 1.56 & -0.47 & N.S. & N.S. & N.S. & N.S. & N.S. & N.S. & N.S. & N.S. & N.S. & N.S. & 5.16 & 0.026 & 2 \\
\hline Lamiaceae & Vitex polygama Cham. & 1.64 & 0.13 & N.S. & N.S. & N.S. & N.S. & 5.04 & 0.027 & N.S. & N.S. & 8.99 & 0.003 & N.S. & N.S. & 3 \\
\hline Lauraceae & Mezilaurus crassiramea (Meisn.) Taub. & 0.85 & 0.49 & N.S. & N.S. & N.S. & N.S. & N.S. & N.S. & N.S. & N.S. & N.S. & N.S. & N.S. & N.S. & 6 \\
\hline Lauraceae & Ocotea corymbosa (Meisn.) Mez & -2.56 & -0.52 & N.S. & N.S. & 17.51 & $<0.001$ & N.S. & N.S. & 23.63 & $<0.001$ & N.S. & N.S. & 5.27 & 0.024 & 10 \\
\hline Lauraceae & Ocotea minarum (Nees \& Mart.) Mez & -2.54 & -1.33 & N.S. & N.S. & 6.93 & 0.010 & 9.86 & 0.002 & 5.55 & 0.021 & N.S. & N.S. & 6.11 & 0.016 & 6 \\
\hline Lauraceae & Ocotea pulchella (Nees \& Mart.) Mez & -2.24 & 0.08 & 4.46 & 0.038 & 9.50 & 0.002 & N.S. & N.S. & 6.97 & 0.010 & N.S. & N.S. & 4.45 & 0.038 & 7 \\
\hline Lecythidaceae & Eschweilera nana (O.Berg) Miers & 1.01 & 1.94 & 4.51 & 0.037 & N.S. & N.S. & N.S. & N.S. & 30.12 & $<0.001$ & N.S. & N.S. & 5.19 & 0.025 & 9 \\
\hline
\end{tabular}




\begin{tabular}{|c|c|c|c|c|c|c|c|c|c|c|c|c|c|c|c|c|}
\hline Família & Espécie & PC1 & PC2 & $\begin{array}{c}\text { SB } \\
\mathbf{F}_{1,78}\end{array}$ & $p$ & $\begin{array}{c}m \\
F_{1,78}\end{array}$ & $p$ & $\begin{array}{c}\mathrm{pH} \\
\mathbf{F}_{1,78}\end{array}$ & $p$ & $\begin{array}{c}\text { Bio05 } \\
F_{1,78} \\
\end{array}$ & $p$ & $\begin{array}{c}\text { Bio23 } \\
F_{1,78} \\
\end{array}$ & $p$ & $\begin{array}{c}\text { Bio30 } \\
F_{1,78} \\
\end{array}$ & $p$ & $\mathbf{N}$ \\
\hline Loganiaceae & Antonia ovata Pohl & 1.05 & 0.74 & N.S. & N.S. & N.S. & N.S. & N.S. & N.S. & N.S. & N.S. & N.S. & N.S. & N.S. & N.S. & 9 \\
\hline Loganiaceae & Strychnos pseudoquina A.St.-Hil. & -1.00 & 0.27 & 10.71 & 0.002 & N.S. & N.S. & 6.89 & 0.010 & N.S. & N.S. & N.S. & N.S. & N.S. & N.S. & 34 \\
\hline Lythraceae & $\begin{array}{l}\text { Lafoensia pacari A.St.-Hil. } \\
\text { Lafoensia vandelliana subsp. replicata (Pohl) }\end{array}$ & -1.47 & 0.19 & N.S. & N.S. & 27.58 & $<0.001$ & N.S. & N.S. & N.S. & N.S. & 5.58 & 0.020 & 12.86 & 0.001 & 22 \\
\hline Lythraceae & Lourteig & 1.83 & 0.16 & N.S. & N.S. & 34.90 & $<0.001$ & 4.55 & 0.036 & 4.37 & 0.039 & N.S. & N.S. & 13.16 & 0.001 & 35 \\
\hline Lythraceae & Physocalymma scaberrimum Pohl & 2.12 & 0.07 & 4.99 & 0.028 & N.S. & N.S. & N.S. & N.S. & N.S. & N.S. & 6.82 & 0.017 & N.S. & N.S. & 11 \\
\hline Malpighiaceae & Banisteriopsis latifolia (A.Juss.) B.Gates & 1.82 & 0.24 & N.S. & N.S. & N.S. & N.S. & N.S. & N.S. & N.S. & N.S. & N.S. & N.S. & N.S. & N.S. & 4 \\
\hline Malpighiaceae & Byrsonima basiloba A.Juss. & -0.67 & 0.98 & 8.43 & 0.005 & N.S. & N.S. & N.S. & N.S. & N.S. & N.S. & N.S. & N.S. & N.S. & N.S. & 11 \\
\hline Malpighiaceae & Byrsonima coccolobifolia Kunth & -0.02 & 0.47 & N.S. & N.S. & 4.54 & 0.036 & N.S. & N.S. & N.S. & N.S. & N.S. & N.S. & N.S. & N.S. & 48 \\
\hline Malpighiaceae & Byrsonima correifolia A.Juss. & 0.47 & 1.17 & N.S. & N.S. & 5.56 & 0.021 & N.S. & N.S. & N.S. & N.S. & 7.58 & 0.007 & N.S. & N.S. & 5 \\
\hline Malpighiaceae & Byrsonima crassifolia (L.) Kunth & 0.92 & 0.04 & N.S. & N.S. & N.S. & N.S. & N.S. & N.S. & N.S. & N.S. & 5.53 & 0.021 & N.S. & N.S. & 7 \\
\hline Malpighiaceae & Byrsonima cydoniifolia A.Juss. & -0.98 & 0.95 & N.S. & N.S. & 7.04 & 0.009 & 6.03 & 0.016 & N.S. & N.S. & N.S. & N.S. & N.S. & N.S. & 6 \\
\hline Malpighiaceae & Byrsonima intermedia A.Juss. & -0.45 & 0.63 & N.S. & N.S. & N.S. & N.S. & N.S. & N.S. & 5.79 & 0.018 & N.S. & N.S. & N.S. & N.S. & 10 \\
\hline Malpighiaceae & Byrsonima pachyphylla A.Juss. & 0.87 & 0.50 & 8.00 & 0.006 & 10.23 & 0.002 & 9.59 & 0.003 & N.S. & N.S. & N.S. & N.S. & 13.36 & $<0.001$ & 56 \\
\hline Malpighiaceae & Byrsonima sericea DC. & -0.87 & 0.32 & N.S. & N.S. & N.S. & N.S. & 7.09 & 0.009 & N.S. & N.S. & 6.97 & 0.009 & 11.17 & 0.001 & 4 \\
\hline Malpighiaceae & Byrsonima verbascifolia (L.) DC. & -0.37 & 0.43 & 6.02 & 0.016 & N.S. & N.S. & N.S. & N.S. & N.S. & N.S. & N.S. & N.S. & N.S. & N.S. & 28 \\
\hline Malpighiaceae & Heteropterys byrsonimiifolia A.Juss. & 1.44 & 0.27 & N.S. & N.S. & N.S. & N.S. & N.S. & N.S. & N.S. & N.S. & 5.07 & 0.270 & N.S. & N.S. & 22 \\
\hline Malvaceae & Apeiba tibourbou Aubl. & 2.59 & 0.64 & N.S. & N.S. & N.S. & N.S. & N.S. & N.S. & N.S. & N.S. & 5.77 & 0.020 & N.S. & N.S. & 2 \\
\hline Malvaceae & Eriotheca gracilipes (K.Schum.) A.Robyns & 0.38 & 0.57 & 4.50 & 0.037 & N.S. & N.S. & N.S. & N.S. & N.S. & N.S. & N.S. & N.S. & N.S. & N.S. & 52 \\
\hline Malvaceae & Eriotheca parvifolia (Mart. \& Zucc.) A.Robyns & 2.33 & 1.11 & 12.19 & 0.001 & N.S. & N.S. & N.S. & N.S. & N.S. & N.S. & N.S. & N.S. & N.S. & N.S. & 9 \\
\hline Malvaceae & Eriotheca pubescens (Mart. \& Zucc.) Schott \& Endl. & -2.79 & 0.04 & N.S. & N.S. & 8.88 & 0.004 & N.S. & N.S. & N.S. & N.S. & N.S. & N.S. & N.S. & N.S. & 7 \\
\hline Malvaceae & Guazuma ulmifolia Lam. & 2.09 & -0.65 & 11.64 & 0.001 & N.S. & N.S. & 7.71 & 0.007 & N.S. & N.S. & 19.47 & $<0.001$ & N.S. & N.S. & 18 \\
\hline Malvaceae & Luehea divaricata Mart. \& Zucc. & -0.49 & -3.67 & 6.56 & 0.012 & N.S. & N.S. & N.S. & N.S. & 4.48 & 0.037 & N.S. & N.S. & N.S. & N.S. & 4 \\
\hline Malvaceae & Luehea grandiflora Mart. \& Zucc. & -1.77 & -0.81 & N.S. & N.S. & 7.83 & 0.006 & N.S. & N.S. & 8.15 & 0.006 & N.S. & N.S. & N.S. & N.S. & 7 \\
\hline Malvaceae & $\begin{array}{l}\text { Luehea paniculata Mart. \& Zucc. } \\
\text { Pseudobombax longiflorum (Mart. \& Zucc.) }\end{array}$ & 1.81 & -0.62 & 19.50 & $<0.001$ & 7.34 & 0.008 & 9.36 & 0.003 & 11.54 & 0.002 & 8.00 & 0.006 & 10.18 & 0.002 & 25 \\
\hline Malvaceae & A.Robyns & 1.65 & -0.68 & 24.35 & $<0.001$ & N.S. & N.S. & N.S. & N.S. & N.S. & N.S. & 6.46 & 0.013 & 4.49 & 0.037 & 31 \\
\hline Malvaceae & $\begin{array}{l}\text { Pseudobombax marginatum (A.St.-Hil.) A. Robyns } \\
\text { Pseudobombax tomentosum (Mart. \& Zucc.) }\end{array}$ & 1.59 & -1.89 & 4.82 & 0.030 & N.S. & N.S. & N.S. & N.S. & N.S. & N.S. & N.S. & N.S. & N.S. & N.S. & 5 \\
\hline Malvaceae & A.Robyns & 1.34 & -0.96 & 11.45 & 0.001 & N.S. & N.S. & 6.03 & 0.016 & 4.35 & 0.040 & N.S. & N.S. & 12.07 & 0.001 & 23 \\
\hline Malvaceae & Sterculia striata A.St.-Hil. \& Naudin & 2.00 & -0.30 & N.S. & N.S. & N.S. & N.S. & N.S. & N.S. & N.S. & N.S. & N.S. & N.S. & N.S. & N.S. & 3 \\
\hline
\end{tabular}




\begin{tabular}{|c|c|c|c|c|c|c|c|c|c|c|c|c|c|c|c|c|}
\hline Família & Espécie & PC1 & PC2 & $\begin{array}{c}\text { SB } \\
F_{1,78}\end{array}$ & $p$ & $\begin{array}{c}m \\
F_{1,78}\end{array}$ & $p$ & $\begin{array}{c}\mathrm{pH} \\
\mathrm{F}_{1,78}\end{array}$ & $p$ & $\begin{array}{c}\text { Bio05 } \\
F_{1,78} \\
\end{array}$ & $p$ & $\begin{array}{c}\text { Bio23 } \\
F_{1,78}\end{array}$ & $p$ & $\begin{array}{c}\text { Bio30 } \\
F_{1,78} \\
\end{array}$ & $p$ & $\mathbf{N}$ \\
\hline Marcgraviaceae & Norantea guianensis Aubl. & -1.51 & 0.55 & N.S. & N.S. & N.S. & N.S. & N.S. & N.S. & N.S. & N.S. & N.S. & N.S. & N.S. & N.S. & 2 \\
\hline Melastomataceae & Miconia albicans (Sw.) Triana & -0.82 & 0.40 & N.S. & N.S. & 7.29 & 0.009 & N.S. & N.S. & 9.55 & 0.003 & 4.78 & 0.032 & N.S. & N.S. & 37 \\
\hline Melastomataceae & Miconia burchellii Triana & -0.49 & -0.19 & N.S. & N.S. & N.S. & N.S. & N.S. & N.S. & N.S. & N.S. & N.S. & N.S. & N.S. & N.S. & 2 \\
\hline Melastomataceae & Miconia fallax DC. & -0.65 & 0.91 & N.S. & N.S. & N.S. & N.S. & N.S. & N.S. & N.S. & N.S. & N.S. & N.S. & N.S. & N.S. & 4 \\
\hline Melastomataceae & Miconia ferruginata DC. & 0.50 & 1.87 & N.S. & N.S. & N.S. & N.S. & N.S. & N.S. & N.S. & N.S. & N.S. & N.S. & N.S. & N.S. & 3 \\
\hline Melastomataceae & Miconia leucocarpa DC. & -3.40 & 0.42 & N.S. & N.S. & 4.22 & 0.040 & N.S. & N.S. & 5.77 & 0.018 & N.S. & N.S. & N.S. & N.S. & 3 \\
\hline Melastomataceae & Miconia ligustroides (DC.) Naudin & -4.77 & 0.15 & N.S. & N.S. & N.S. & N.S. & 5.55 & 0.021 & N.S. & N.S. & N.S. & N.S. & N.S. & N.S. & 2 \\
\hline Melastomataceae & Miconia macrothyrsa Benth. & -0.38 & 0.73 & N.S. & N.S. & N.S. & N.S. & N.S. & N.S. & N.S. & N.S. & N.S. & N.S. & N.S. & N.S. & 3 \\
\hline Melastomataceae & Miconia rubiginosa (Bonpl.) DC. & -4.77 & 0.15 & N.S. & N.S. & N.S. & N.S. & 5.55 & 0.020 & N.S. & N.S. & N.S. & N.S. & N.S. & N.S. & 2 \\
\hline Melastomataceae & Miconia stenostachya DC. & 2.25 & 1.23 & N.S. & N.S. & 4.41 & 0.039 & N.S. & N.S. & N.S. & N.S. & 9.01 & 0.004 & N.S. & N.S. & 5 \\
\hline Melastomataceae & Mouriri elliptica Mart. & 1.95 & 0.43 & N.S. & N.S. & 13.46 & $<0.001$ & N.S. & N.S. & 7.55 & 0.007 & N.S. & N.S. & N.S. & N.S. & 25 \\
\hline Melastomataceae & Mouriri pusa Gardner & 1.70 & 0.95 & N.S. & N.S. & 6.29 & 0.014 & N.S. & N.S. & 11.72 & 0.001 & 9.02 & 0.004 & N.S. & N.S. & 19 \\
\hline Meliaceae & Cabralea canjerana (Vell.) Mart. & -3.20 & -0.73 & N.S. & N.S. & 6.22 & 0.015 & N.S. & N.S. & 4.49 & 0.037 & N.S. & N.S. & N.S. & N.S. & 2 \\
\hline Meliaceae & Trichilia pallida Sw. & -0.88 & -2.55 & 8.51 & 0.005 & 11.18 & 0.001 & N.S. & N.S. & 23.22 & $<0.001$ & N.S. & N.S. & N.S. & N.S. & 7 \\
\hline Moraceae & Brosimum gaudichaudii Trécul & 0.48 & 0.08 & N.S. & N.S. & N.S. & N.S. & 4.85 & 0.030 & N.S. & N.S. & N.S. & N.S. & N.S. & N.S. & 50 \\
\hline Moraceae & Ficus enormis Mart. ex Miq. & -1.27 & -0.76 & N.S. & N.S. & N.S. & N.S. & N.S. & N.S. & N.S. & N.S. & N.S. & N.S. & N.S. & N.S. & 3 \\
\hline Myristicaceae & Virola sebifera Aubl. & -1.18 & 0.09 & N.S. & N.S. & 8.44 & 0.005 & N.S. & N.S. & N.S. & N.S. & N.S. & N.S. & N.S. & N.S. & 15 \\
\hline Myrtaceae & Blepharocalyx salicifolius (Kunth) O.Berg & -4.53 & -0.45 & N.S. & N.S. & N.S. & N.S. & N.S. & N.S. & N.S. & N.S. & N.S. & N.S. & N.S. & N.S. & 2 \\
\hline Myrtaceae & Campomanesia adamantium (Cambess.) O.Berg & -3.40 & -1.22 & N.S. & N.S. & N.S. & N.S. & 9.09 & 0.003 & 6.98 & 0.010 & N.S. & N.S. & N.S. & N.S. & 2 \\
\hline Myrtaceae & Campomanesia velutina (Cambess.) O.Berg & -0.36 & -2.27 & N.S. & N.S. & N.S. & N.S. & N.S. & N.S. & 4.53 & 0.036 & N.S. & N.S. & N.S. & N.S. & 4 \\
\hline Myrtaceae & Eugenia aurata O.Berg & -1.55 & 0.12 & N.S. & N.S. & 5.80 & 0.018 & N.S. & N.S. & N.S. & N.S. & N.S. & N.S. & N.S. & N.S. & 12 \\
\hline Myrtaceae & Eugenia dysenterica DC. & 1.01 & 0.11 & N.S. & N.S. & 7.17 & 0.009 & 5.61 & 0.020 & N.S. & N.S. & N.S. & N.S. & 29.74 & $<0.001$ & 43 \\
\hline Myrtaceae & Eugenia punicifolia (Kunth) DC. & -2.62 & 0.50 & N.S. & N.S. & 11.62 & 0.001 & N.S. & N.S. & 9.29 & 0.003 & N.S. & N.S. & 13.25 & $<0.001$ & 11 \\
\hline Myrtaceae & Myrcia albotomentosa DC. & 0.72 & -0.23 & N.S. & N.S. & N.S. & N.S. & N.S. & N.S. & N.S. & N.S. & N.S. & N.S. & N.S. & N.S. & 8 \\
\hline Myrtaceae & Myrcia bella Cambess. & -3.57 & 0.25 & N.S. & N.S. & 5.79 & 0.018 & 6.52 & 0.013 & 5.23 & 0.025 & N.S. & N.S. & N.S. & N.S. & 4 \\
\hline Myrtaceae & Myrcia fenzliana O.Berg & -1.71 & 1.88 & 4.61 & 0.035 & N.S. & N.S. & N.S. & N.S. & N.S. & N.S. & N.S. & N.S. & N.S. & N.S. & 2 \\
\hline Myrtaceae & Myrcia guianensis (Aubl.) DC. & 0.28 & -0.09 & N.S. & N.S. & N.S. & N.S. & N.S. & N.S. & N.S. & N.S. & 7.28 & 0.009 & N.S. & N.S. & 20 \\
\hline Myrtaceae & Myrcia lanuginosa O.Berg & 2.27 & -0.81 & 4.22 & 0.043 & N.S. & N.S. & N.S. & N.S. & N.S. & N.S. & N.S. & N.S. & N.S. & N.S. & 4 \\
\hline Myrtaceae & Myrcia selloi (Spreng.) N.Silveira & 0.84 & 0.79 & N.S. & N.S. & N.S. & N.S. & 5.33 & 0.023 & N.S. & N.S. & 9.51 & 0.003 & N.S. & N.S. & 3 \\
\hline
\end{tabular}




\begin{tabular}{|c|c|c|c|c|c|c|c|c|c|c|c|c|c|c|c|c|}
\hline Família & Espécie & PC1 & PC2 & $\begin{array}{c}\text { SB } \\
\mathbf{F}_{1,78} \\
\end{array}$ & $p$ & $\begin{array}{c}m \\
F_{1,78} \\
\end{array}$ & $p$ & $\begin{array}{c}\mathrm{pH} \\
\mathrm{F}_{1,78} \\
\end{array}$ & $p$ & $\begin{array}{c}\text { Bio05 } \\
F_{1,78}\end{array}$ & $p$ & $\begin{array}{c}\text { Bio23 } \\
F_{1,78} \\
\end{array}$ & $p$ & $\begin{array}{c}\text { Bio30 } \\
F_{1,78} \\
\end{array}$ & $p$ & $\mathbf{N}$ \\
\hline Myrtaceae & Myrcia splendens (Sw.) DC. & 0.90 & 0.18 & N.S. & N.S. & N.S. & N.S. & N.S. & N.S. & N.S. & N.S. & 18.36 & $<0.001$ & N.S. & N.S. & 43 \\
\hline Myrtaceae & Myrcia tomentosa (Aubl.) DC. & -1.05 & -0.34 & N.S. & N.S. & 11.85 & 0.001 & N.S. & N.S. & 8.59 & 0.004 & N.S. & N.S. & N.S. & N.S. & 15 \\
\hline Myrtaceae & Myrcia variabilis DC. & -1.77 & 0.13 & 8.99 & 0.004 & 12.52 & 0.007 & 10.90 & 0.001 & 7.84 & 0.006 & N.S. & N.S. & 5.96 & 0.017 & 8 \\
\hline Myrtaceae & Psidium laruotteanum Cambess. & -1.62 & 1.94 & 9.16 & 0.003 & N.S. & N.S. & N.S. & N.S. & N.S. & N.S. & N.S. & N.S. & N.S. & N.S. & 3 \\
\hline Myrtaceae & Psidium myrtoides O.Berg & 1.15 & 0.67 & 8.47 & 0.004 & 18.90 & $<0.001$ & 5.68 & 0.019 & 5.32 & 0.023 & N.S. & N.S. & 11.78 & $<0.001$ & 32 \\
\hline Myrtaceae & Psidium pohlianum O.Berg & -2.62 & 1.19 & 4.28 & 0.040 & 4.72 & 0.032 & N.S. & N.S. & N.S. & N.S. & N.S. & N.S. & N.S. & N.S. & 3 \\
\hline Myrtaceae & Psidium salutare (Kunth) O.Berg & -1.20 & 0.91 & N.S. & N.S. & 4.12 & 0.045 & N.S. & N.S. & N.S. & N.S. & 5.04 & 0.027 & N.S. & N.S. & 3 \\
\hline Nyctaginaceae & Guapira graciliflora (Mart. ex Schmidt) Lundell & 0.71 & 0.65 & N.S. & N.S. & N.S. & N.S. & N.S. & N.S. & N.S. & N.S. & N.S. & N.S. & N.S. & N.S. & 24 \\
\hline Nyctaginaceae & Guapira noxia (Netto) Lundell & -0.84 & -0.02 & N.S. & N.S. & 27.01 & $<0.001$ & N.S. & N.S. & 10.20 & 0.002 & N.S. & N.S. & N.S. & N.S. & 30 \\
\hline Nyctaginaceae & Guapira opposita (Vell.) Reitz & -4.19 & -1.22 & N.S. & N.S. & N.S. & N.S. & N.S. & N.S. & N.S. & N.S. & 4.76 & 0.032 & 7.46 & 0.008 & 3 \\
\hline Nyctaginaceae & Neea theifera Oerst. & 0.54 & 0.10 & N.S. & N.S. & N.S. & N.S. & 4.12 & 0.046 & N.S. & N.S. & N.S. & N.S. & N.S. & N.S. & 36 \\
\hline Ochnaceae & Ouratea castaneifolia (DC.) Engl. & 0.03 & -0.81 & N.S. & N.S. & 12.71 & 0.001 & 8.73 & 0.004 & N.S. & N.S. & N.S. & N.S. & N.S. & N.S. & 22 \\
\hline Ochnaceae & Ouratea hexasperma (A.St.-Hil.) Baill. & 0.71 & 0.66 & 8.58 & 0.004 & N.S. & N.S. & 5.47 & 0.021 & N.S. & N.S. & N.S. & N.S. & N.S. & N.S. & 53 \\
\hline Ochnaceae & Ouratea spectabilis (Mart.) Engl. & -0.19 & 0.55 & N.S. & N.S. & N.S. & N.S. & N.S. & N.S. & N.S. & N.S. & N.S. & N.S. & N.S. & N.S. & 22 \\
\hline Olacaceae & Heisteria ovata Benth. & 0.28 & 0.49 & 6.18 & 0.015 & 5.05 & 0.027 & N.S. & N.S. & N.S. & N.S. & 13.19 & $<0.001$ & 7.29 & 0.008 & 22 \\
\hline Olacaceae & Ximenia americana L. & 1.89 & -0.24 & N.S. & N.S. & N.S. & N.S. & N.S. & N.S. & N.S. & N.S. & N.S. & N.S. & N.S. & N.S. & 3 \\
\hline Opiliaceae & Agonandra brasiliensis Miers ex Benth. \& Hook.f. & 0.51 & 0.04 & N.S. & N.S. & N.S. & N.S. & 5.61 & 0.020 & N.S. & N.S. & N.S. & N.S. & N.S. & N.S. & 48 \\
\hline Peraceae & Pera glabrata (Schott) Poepp. ex Baill. & -1.73 & -0.24 & N.S. & N.S. & 20.32 & $<0.001$ & N.S. & N.S. & 11.33 & 0.001 & N.S. & N.S. & N.S. & N.S. & 12 \\
\hline Polygonaceae & Coccoloba mollis Casar. & 0.46 & -0.62 & N.S. & N.S. & N.S. & N.S. & 4.80 & 0.031 & N.S. & N.S. & N.S. & N.S. & N.S. & N.S. & 12 \\
\hline Primulaceae & Cybianthus detergens Mart. & 0.62 & -0.63 & N.S. & N.S. & N.S. & N.S. & N.S. & N.S. & N.S. & N.S. & N.S. & N.S. & N.S. & N.S. & 2 \\
\hline Primulaceae & Myrsine guianensis (Aubl.) Kuntze & -2.59 & -0.13 & N.S. & N.S. & 4.11 & 0.046 & N.S. & N.S. & N.S. & N.S. & N.S. & N.S. & N.S. & N.S. & 7 \\
\hline Primulaceae & Myrsine umbellata Mart. & -1.64 & -0.06 & 5.66 & 0.020 & 13.09 & 0.001 & 11.72 & 0.001 & 8.37 & 0.005 & N.S. & N.S. & N.S. & N.S. & 8 \\
\hline Proteaceae & Euplassa inaequalis (Pohl) Engl. & 2.00 & 0.03 & N.S. & N.S. & N.S. & N.S. & N.S. & N.S. & N.S. & N.S. & N.S. & N.S. & 4.98 & 0.028 & 9 \\
\hline Proteaceae & Roupala montana Aubl. & 0.13 & -0.08 & N.S. & N.S. & 4.12 & 0.046 & N.S. & N.S. & N.S. & N.S. & 9.71 & 0.003 & N.S. & N.S. & 48 \\
\hline Rhamnaceae & Rhamnidium elaeocarpum Reissek & 1.88 & -1.99 & 18.04 & $<0.001$ & N.S. & N.S. & 15.84 & $<0.001$ & N.S. & N.S. & N.S. & N.S. & 6.52 & 0.013 & 14 \\
\hline Rubiaceae & Alibertia edulis (Rich.) A.Rich. & 0.90 & -0.42 & 7.47 & 0.008 & N.S. & N.S. & N.S. & N.S. & N.S. & N.S. & N.S. & N.S. & N.S. & N.S. & 28 \\
\hline Rubiaceae & Amaioua guianensis Aubl. & -4.77 & 0.15 & N.S. & N.S. & N.S. & N.S. & 5.50 & 0.020 & N.S. & N.S. & N.S. & N.S. & N.S. & N.S. & 2 \\
\hline Rubiaceae & Chomelia obtusa Cham. \& Schltdl. & 0.36 & -0.17 & N.S. & N.S. & N.S. & N.S. & N.S. & N.S. & N.S. & N.S. & N.S. & N.S. & N.S. & N.S. & 5 \\
\hline Rubiaceae & Chomelia pohliana Müll.Arg. & 2.32 & -0.13 & N.S. & N.S. & N.S. & N.S. & N.S. & N.S. & N.S. & N.S. & N.S. & N.S. & N.S. & N.S. & 5 \\
\hline
\end{tabular}




\begin{tabular}{|c|c|c|c|c|c|c|c|c|c|c|c|c|c|c|c|c|}
\hline Família & Espécie & PC1 & PC2 & $\begin{array}{c}\text { SB } \\
F_{1,78} \\
\end{array}$ & $p$ & $\begin{array}{c}m \\
F_{1,78}\end{array}$ & $p$ & $\begin{array}{c}\mathrm{pH} \\
\mathrm{F}_{1,78}\end{array}$ & $p$ & $\begin{array}{c}\text { Bio05 } \\
F_{1,78}\end{array}$ & $p$ & $\begin{array}{c}\text { Bio23 } \\
F_{1,78} \\
\end{array}$ & $p$ & $\begin{array}{c}\text { Bio30 } \\
F_{1,78}\end{array}$ & $p$ & $\mathbf{N}$ \\
\hline Rubiaceae & Chomelia ribesioides Benth. ex A.Gray & 2.71 & 0.38 & N.S. & N.S. & N.S. & N.S. & N.S. & N.S. & N.S. & N.S. & N.S. & N.S. & N.S. & N.S. & 2 \\
\hline Rubiaceae & Cordiera elliptica (Cham.) Kuntze & -0.53 & -0.37 & N.S. & N.S. & N.S. & N.S. & N.S. & N.S. & N.S. & N.S. & N.S. & N.S. & 10.67 & 0.002 & 13 \\
\hline Rubiaceae & Cordiera macrophylla (K.Schum.) Kuntze & -0.74 & 0.48 & N.S. & N.S. & N.S. & N.S. & N.S. & N.S. & N.S. & N.S. & N.S. & N.S. & N.S. & N.S. & 6 \\
\hline Rubiaceae & Cordiera sessilis (Vell.) Kuntze & -0.24 & 1.00 & N.S. & N.S. & 8.08 & 0.006 & N.S. & N.S. & N.S. & N.S. & N.S. & N.S. & 7.06 & 0.009 & 9 \\
\hline Rubiaceae & Coussarea hydrangeifolia (Benth.) Müll.Arg. & 0.42 & 0.22 & N.S. & N.S. & N.S. & N.S. & N.S. & N.S. & N.S. & N.S. & 9.84 & 0.002 & N.S. & N.S. & 23 \\
\hline Rubiaceae & Faramea hyacinthina Mart. & -0.65 & -1.55 & N.S. & N.S. & N.S. & N.S. & N.S. & N.S. & N.S. & N.S. & N.S. & N.S. & N.S. & N.S. & 2 \\
\hline Rubiaceae & Ferdinandusa elliptica (Pohl) Pohl & 2.00 & 0.37 & N.S. & N.S. & N.S. & N.S. & N.S. & N.S. & 5.85 & 0.017 & 4.99 & 0.028 & N.S. & N.S. & 15 \\
\hline Rubiaceae & Genipa americana L. & 1.01 & -0.46 & N.S. & N.S. & N.S. & N.S. & N.S. & N.S. & N.S. & N.S. & N.S. & N.S. & N.S. & N.S. & 2 \\
\hline Rubiaceae & Guettarda viburnoides Cham. \& Schltdl. & 1.82 & -1.05 & 16.27 & 0.001 & N.S. & N.S. & 7.32 & 0.008 & N.S. & N.S. & 9.35 & 0.003 & N.S. & N.S. & 21 \\
\hline Rubiaceae & Palicourea rigida Kunth & -0.19 & 0.50 & N.S. & N.S. & N.S. & N.S. & N.S. & N.S. & N.S. & N.S. & N.S. & N.S. & N.S. & N.S. & 21 \\
\hline Rubiaceae & Rudgea viburnoides (Cham.) Benth. & -0.68 & -0.61 & N.S. & N.S. & 15.87 & $<0.001$ & N.S. & N.S. & 6.44 & 0.013 & N.S. & N.S. & N.S. & N.S. & 24 \\
\hline Rubiaceae & Tocoyena formosa (Cham. \& Schltdl.) K.Schum. & 0.89 & 0.50 & N.S. & N.S. & N.S. & N.S. & N.S. & N.S. & 5.24 & 0.025 & 9.28 & 0.003 & N.S. & N.S. & 53 \\
\hline Rutaceae & Zanthoxylum rhoifolium Lam. & 0.83 & -0.61 & N.S. & N.S. & N.S. & N.S. & N.S. & N.S. & N.S. & N.S. & N.S. & N.S. & N.S. & N.S. & 3 \\
\hline Rutaceae & Zanthoxylum riedelianum Engl. & 0.93 & -0.81 & N.S. & N.S. & N.S. & N.S. & N.S. & N.S. & N.S. & N.S. & N.S. & N.S. & N.S. & N.S. & 10 \\
\hline Rutaceae & Zanthoxylum rigidum Humb. \& Bonpl. exWilld. & -3.76 & -4.26 & N.S. & N.S. & N.S. & N.S. & N.S. & N.S. & N.S. & N.S. & N.S. & N.S. & 14.00 & $<0.001$ & 2 \\
\hline Salicaceae & Casearia arborea (Rich.) Urb. & -0.71 & 0.60 & N.S. & N.S. & N.S. & N.S. & N.S. & N.S. & N.S. & N.S. & 10.73 & 0.002 & N.S. & N.S. & 5 \\
\hline Salicaceae & Casearia grandiflora Cambess. & 0.63 & 0.81 & N.S. & N.S. & N.S. & N.S. & N.S. & N.S. & N.S. & N.S. & N.S. & N.S. & N.S. & N.S. & 4 \\
\hline Salicaceae & Casearia rupestris Eichler & 3.09 & -6.21 & 7.36 & 0.008 & N.S. & N.S. & 6.73 & 0.011 & N.S. & N.S. & N.S. & N.S. & N.S. & N.S. & 2 \\
\hline Salicaceae & Casearia sylvestris Sw. & 0.61 & -0.39 & 14.96 & $<0.001$ & 6.81 & 0.018 & N.S. & N.S. & N.S. & N.S. & N.S. & N.S. & N.S. & N.S. & 60 \\
\hline Sapindaceae & Cupania vernalis Cambess. & -1.49 & -1.84 & N.S. & N.S. & N.S. & N.S. & 4.89 & 0.029 & 10.60 & 0.002 & N.S. & N.S. & N.S. & N.S. & 4 \\
\hline Sapindaceae & Dilodendron bipinnatum Radlk. & 1.56 & -1.48 & 7.35 & 0.008 & N.S. & N.S. & 5.16 & 0.026 & N.S. & N.S. & N.S. & N.S. & N.S. & N.S. & 12 \\
\hline Sapindaceae & Magonia pubescens A.St.-Hil. & 1.55 & -0.55 & 43.52 & $<0.001$ & 5.78 & 0.018 & N.S. & N.S. & 10.10 & 0.002 & N.S. & N.S. & N.S. & N.S. & 42 \\
\hline Sapindaceae & Matayba guianensis Aubl. & 0.35 & -0.33 & N.S. & N.S. & N.S. & N.S. & N.S. & N.S. & N.S. & N.S. & 5.14 & 0.026 & N.S. & N.S. & 22 \\
\hline Sapotaceae & Chrysophyllum marginatum (Hook. \& Arn.) Radlk. & -1.94 & -3.03 & N.S. & N.S. & N.S. & N.S. & 4.56 & 0.036 & N.S. & N.S. & N.S. & N.S. & 7.29 & 0.008 & 4 \\
\hline Sapotaceae & Pouteria gardneri (Mart. \& Miq.) Baehni & -0.44 & -4.52 & N.S. & N.S. & N.S. & N.S. & N.S. & N.S. & N.S. & N.S. & N.S. & N.S. & N.S. & N.S. & 2 \\
\hline Sapotaceae & Pouteria ramiflora (Mart.) Radlk. & 0.56 & 0.66 & 4.56 & 0.036 & 6.32 & 0.014 & N.S. & N.S. & 6.25 & 0.015 & 4.31 & 0.014 & N.S. & N.S. & 56 \\
\hline Sapotaceae & Pouteria torta (Mart.) Radlk. & -0.44 & 0.38 & 13.08 & 0.001 & N.S. & N.S. & 4.17 & 0.044 & 8.12 & 0.006 & 7.53 & 0.007 & N.S. & N.S. & 25 \\
\hline Simaroubaceae & Simaba ferruginea A.St.-Hil. & 1.22 & 1.35 & 5.69 & 0.019 & 17.04 & $<0.001$ & N.S. & N.S. & N.S. & N.S. & 18.35 & $<0.001$ & N.S. & N.S. & 8 \\
\hline Simaroubaceae & Simarouba versicolor A.St.-Hil. & 1.46 & 0.25 & N.S. & N.S. & 16.15 & $<0.001$ & N.S. & N.S. & 4.83 & 0.031 & N.S. & N.S. & N.S. & N.S. & 39 \\
\hline
\end{tabular}




\begin{tabular}{|c|c|c|c|c|c|c|c|c|c|c|c|c|c|c|c|c|}
\hline Família & Espécie & PC1 & PC2 & $\begin{array}{c}\text { SB } \\
F_{1,78} \\
\end{array}$ & $p$ & $\begin{array}{c}\mathbf{m} \\
\mathrm{F}_{1,78} \\
\end{array}$ & $p$ & $\begin{array}{c}\text { pH } \\
\mathrm{F}_{1,78} \\
\end{array}$ & $p$ & $\begin{array}{c}\text { Bio05 } \\
F_{1,78}\end{array}$ & $p$ & $\begin{array}{c}\text { Bio23 } \\
F_{1,78} \\
\end{array}$ & $p$ & $\begin{array}{c}\text { Bio30 } \\
F_{1,78} \\
\end{array}$ & $p$ & $\mathbf{N}$ \\
\hline Siparunaceae & Siparuna guianensis Aubl. & -0.25 & 0.39 & N.S. & N.S. & 4.87 & 0.030 & N.S. & N.S. & N.S. & N.S. & 11.64 & 0.001 & N.S. & N.S. & 26 \\
\hline Solanaceae & Solanum lycocarpum A.St.-Hil. & -1.33 & 0.19 & N.S. & N.S. & N.S. & N.S. & N.S. & N.S. & N.S. & N.S. & N.S. & N.S. & N.S. & N.S. & 4 \\
\hline Styracaceae & Styrax camporum Pohl & -1.68 & -0.49 & N.S. & N.S. & 20.33 & $<0.001$ & N.S. & N.S. & 10.96 & 0.001 & N.S. & N.S. & N.S. & N.S. & 11 \\
\hline Styracaceae & Styrax ferrugineus Nees \& Mart. & -1.78 & 0.39 & 8.43 & 0.005 & 20.43 & $<0.001$ & N.S. & N.S. & 11.12 & 0.001 & N.S. & N.S. & N.S. & N.S. & 14 \\
\hline Symplocaceae & Symplocos nitens (Pohl) Benth. & -1.38 & -1.26 & N.S. & N.S. & 9.68 & 0.003 & 10.66 & 0.002 & 8.87 & 0.004 & N.S. & N.S. & N.S. & N.S. & 7 \\
\hline Symplocaceae & Symplocos platyphylla (Pohl) Benth. & -2.09 & 0.57 & 6.46 & 0.013 & 5.35 & 0.023 & 8.36 & 0.005 & N.S. & N.S. & N.S. & N.S. & N.S. & N.S. & 4 \\
\hline Symplocaceae & Symplocos pubescens Klotzsch exBenth. & -2.76 & 0.87 & 4.43 & 0.038 & N.S. & N.S. & 4.29 & 0.041 & N.S. & N.S. & N.S. & N.S. & N.S. & N.S. & 2 \\
\hline Urticaceae & Cecropia pachystachya Trécul & 0.76 & 0.39 & N.S. & N.S. & N.S. & N.S. & N.S. & N.S. & N.S. & N.S. & N.S. & N.S. & N.S. & N.S. & 12 \\
\hline Vochysiaceae & Callisthene fasciculata Mart. & 1.71 & -0.82 & 17.10 & $<0.001$ & N.S. & N.S. & N.S. & N.S. & 9.86 & 0.002 & N.S. & N.S. & N.S. & N.S. & 24 \\
\hline Vochysiaceae & Callisthene hassleri Briq. & 1.54 & -0.06 & N.S. & N.S. & N.S. & N.S. & N.S. & N.S. & N.S. & N.S. & N.S. & N.S. & N.S. & N.S. & 8 \\
\hline Vochysiaceae & Callisthene major Mart. \& Zucc. & 0.87 & 0.37 & N.S. & N.S. & N.S. & N.S. & N.S. & N.S. & N.S. & N.S. & N.S. & N.S. & N.S. & N.S. & 4 \\
\hline Vochysiaceae & Qualea cordata (Mart.) Spreng. & -2.77 & 0.46 & N.S. & N.S. & N.S. & N.S. & N.S. & N.S. & 5.48 & 0.217 & N.S. & N.S. & N.S. & N.S. & 3 \\
\hline Vochysiaceae & Qualea dichotoma (Mart.) Warm. & -2.26 & 0.31 & N.S. & N.S. & 5.13 & 0.026 & N.S. & N.S. & 4.71 & 0.019 & N.S. & N.S. & N.S. & N.S. & 8 \\
\hline Vochysiaceae & Qualea grandiflora Mart. & 0.34 & 0.16 & N.S. & N.S. & N.S. & N.S. & 4.56 & 0.036 & N.S. & N.S. & N.S. & N.S. & 5.65 & 0.019 & 74 \\
\hline Vochysiaceae & Qualea multiflora Mart. & 0.25 & -0.06 & N.S. & N.S. & 6.85 & 0.010 & N.S. & N.S. & N.S. & N.S. & N.S. & N.S. & N.S. & N.S. & 45 \\
\hline Vochysiaceae & Qualea parviflora Mart. & 0.46 & 0.26 & N.S. & N.S. & N.S. & N.S. & N.S. & N.S. & 7.08 & 0.009 & N.S. & N.S. & N.S. & N.S. & 77 \\
\hline Vochysiaceae & Salvertia convallariodora A.St.-Hil. & 0.48 & 0.46 & 6.58 & 0.012 & N.S. & N.S. & N.S. & N.S. & N.S. & N.S. & 7.46 & 0.008 & N.S. & N.S. & 54 \\
\hline Vochysiaceae & Vochysia cinnamomea Pohl & 1.54 & 1.05 & N.S. & N.S. & N.S. & N.S. & N.S. & N.S. & 5.98 & 0.017 & 18.32 & $<0.001$ & N.S. & N.S. & 9 \\
\hline Vochysiaceae & Vochysia elliptica Mart. & -1.09 & 0.84 & 11.57 & 0.001 & N.S. & N.S. & N.S. & N.S. & N.S. & N.S. & N.S. & N.S. & N.S. & N.S. & 9 \\
\hline Vochysiaceae & Vochysia gardneri Warm. & 0.74 & 1.12 & 8.07 & 0.006 & 14.47 & $<0.001$ & N.S. & N.S. & N.S. & N.S. & 8.88 & 0.004 & 6.80 & 0.011 & 16 \\
\hline Vochysiaceae & Vochysia haenkeana Mart. & 0.45 & 1.38 & N.S. & N.S. & N.S. & N.S. & 4.07 & 0.047 & 9.52 & 0.003 & 6.75 & 0.011 & N.S. & N.S. & 7 \\
\hline Vochysiaceae & Vochysia rufa Mart. & -0.27 & 0.68 & 4.41 & 0.038 & 5.20 & 0.025 & N.S. & N.S. & N.S. & N.S. & N.S. & N.S. & N.S. & N.S. & 28 \\
\hline Vochysiaceae & Vochysia thyrsoidea Pohl & -2.61 & -0.93 & N.S. & N.S. & N.S. & N.S. & N.S. & N.S. & N.S. & N.S. & N.S. & N.S. & N.S. & N.S. & 6 \\
\hline Vochysiaceae & Vochysia tucanorum Mart. & -1.72 & 0.28 & N.S. & N.S. & 16.50 & $<0.001$ & N.S. & N.S. & 6.61 & 0.012 & N.S. & N.S. & N.S. & N.S. & 15 \\
\hline
\end{tabular}


2124 